\title{
Modulating Synthetic Pathways in Megasynthases
}

\author{
Dissertation \\ zur Erlangung des Doktorgrades \\ der Naturwissenschaften
}

\begin{abstract}
vorgelegt beim Fachbereich 14
Biochemie, Chemie und Pharmazie

der Johann Wolfgang Goethe-Universität

in Frankfurt am Main
\end{abstract}

von

Franziska Stegemann

aus Frankfurt am Main

Frankfurt 2021

(D30) 

vom Fachbereich Biochemie, Chemie und Pharmazie der

Johann Wolfgang Goethe-Universität als Dissertation angenommen.

Dekan: Prof. Dr. Clemens Glaubitz

Gutachter: Prof. Dr. Martin Grininger

Prof. Dr. Stefan Knapp

Datum der Disputation: 21.09.2021 



\section{Vorwort}

Teile der vorliegenden Arbeit wurden vorab zur Publikation akzeptiert in: F. Stegemann, M. Grininger, "Transacylation Kinetics in Fatty Acid and Polyketide Synthases and its Sensitivity to Point Mutations", ChemCatChem (2021). ${ }^{[1]}$ Eine detaillierte Auflistung der Inhalte, mein eigener Anteil, Copyright sowie Creative-Commons-Lizenzen sind im Anhang unter dem Abschnitt "Statement of Personal Contributions" dargestellt.

In die vorliegende Arbeit sind die Ergebnisse verschiedener Bachelor- und Master-Abschlussarbeiten des FB14 eingegangen, die von mir betreut wurden. Eine detaillierte Auflistung findet sich ebenfalls im Anhang unter dem Abschnitt "Statement of Personal Contributions". Zusätzlich ist an geeigneten Stellen auf entsprechende Arbeiten verwiesen. 



\section{Zusammenfassung}

Polyketide sind eine Klasse wichtiger Naturstoffe, die aufgrund ihrer nützlichen Eigenschaften in vielen Bereichen der Medizin als Pharmazeutika Anwendung finden. [2] Ihr Wirkspektrum reicht von antibakteriellen und antimykotischen über immunsuppressive bis hin zu antitumoralen Eigenschaften. [3] Diese funktionelle Vielfalt ist auf ihre strukturelle Diversität zurückzuführen. Polyketide können äußerst komplexe Strukturen aufweisen und eine Vielzahl verschiedener funktioneller Gruppen tragen. Inspiriert von der Natur und angetrieben vom Wirkverlust zahlreicher Pharmazeutika sowie auftretenden Resistenzen wird häufig versucht, modifizierte oder neue Naturstoffe zu generieren. Doch wie werden diese komplexen und vielfältigen Moleküle in der Natur hergestellt?

Große Enzymkomplexe, die sogenannten Polyketidsynthasen (PKSs), führen die Biosynthese von Polyketiden durch. ${ }^{4]}$ Auch wenn ihre Komplexität anderes erwarten ließe, werden Polyketide aus einfachen Acyleinheiten schrittweise aufgebaut. Verschiedene Enzyme der PKSs sind für Kondensation, Modifikation und Transport der Substrate und Intermediate zuständig. Abhängig von der Anordnung dieser Enzyme wird zwischen verschiedenen PKS-Typen unterschieden. Die vorliegende Thesis befasst sich ausschließlich mit Typ I-PKS-Systemen, die ihre enzymatischen Funktionen als Domänen auf einer oder mehreren Polypeptidketten tragen. Der Begriff PKS bezieht sich im Folgenden auf Typ I-PKSs.

In allen PKSs schleust die Domäne Acyltransferase (AT) die kleinen Acylsubstrate in den Enzymkomplex ein. Genauer gesagt belädt die AT das Acyl-Carrier-Protein (ACP) mit Substraten, das diese wiederum zu den weiteren katalytischen Domänen zur Prozessierung transportiert. Manche PKSs nutzen ihre Domänen mehrfach in repetitiven Zyklen und werden deshalb iterative PKSs genannt. Andere PKSs enthalten mehrere Domänensätze und nutzen jede Domäne nur genau ein Mal für die Herstellung eines Polyketids. Diese Domänensätze werden als Module bezeichnet und die zugehörigen PKSs deshalb modulare PKSs genannt. PKSs oder PKSModule bestehen aus mindestens drei Domänen, die zur Kondensation der Acylsubstrate nötig sind - AT, ACP und $\beta$-Ketoacylsynthase (KS). ${ }^{[5]}$ Drei weitere Domänen können diesen Minimalsatz erweitern und das Substrat modifizieren. Die Domänenzusammensetzung bestimmt den Grad der Substratmodifikation (keine, partielle oder vollständige Reduktion) und somit die funktionelle Vielfalt im resultierenden Polyketid.

Polyketide und Fettsäuren ähneln sich auf den ersten Blick nur wenig. Dennoch sind ihre Biosynthesewege eng miteinander verwandt. ${ }^{[3,6]}$ Fettsäuren werden von Festtäuresynthasen (FASs) hergestellt, die viele strukturelle sowie funktionelle Eigenschaften mit PKSs teilen. [3] 
FASs bestehen aus den gleichen Domänen, die die gleichen Reaktionen durchführen, um aus kleinen Acylsubstraten Fettsäuren herzustellen. Im Gegensatz zu PKSs enthalten FASs jedoch immer einen kompletten Domänensatz, der iterativ und vollständig reduzierend arbeitet.

FASs sind insgesamt gut in ihrer Struktur und Arbeitsweise verstanden, ${ }^{[7-9]}$ während über PKSs verhältnismäßig wenig bekannt ist. Die vorliegende Thesis beschäftigt sich mit der detaillierten Charakterisierung der AT verschiedener PKSs in ihrer Substratselektivität, ihrer AT-ACP Domänen-Domänen-Interaktion und ihren enzymkinetischen Eigenschaften. Der Vergleich mit enzymkinetischen Daten zu FASs liefert die folgenden Erkenntnisse:

1. ATs der PKSs erscheinen grundsätzlich langsamer als ATs der FASs. Dies könnte auf deren unterschiedliche Biosynthesewege zurückzuführen sein. Fettsäuren sind für den Organismus essentiell und erfüllen wichtige physiologische Aufgaben, u. a. als Bausteine für Membranen, als Energiequelle oder als Botenstoffe in Signalkaskaden. ${ }^{[10-12]}$ Diese vielfältigen Funktionen erfordern hohe Mengen an Fettsäuren, während Polyketide als Sekundärstoffe schon bei niedrigeren Konzentrationen wirken können.

2. Die langsameren ATs der modularen PKSs zeigen sogar in Abwesenheit der nativen Substrate keine Aktivität gegenüber den getesteten nicht-nativen Substraten. Dies steht im Gegensatz zu den schnelleren ATs von iterativen PKSs und FASs, die auch nichtnative Substrate auf ihr jeweiliges ACP laden. ATs modularer PKSs zeigen demnach als einzige eine hohe Substratspezifität in ihrer Beladungsfunktion und erfüllen wohl die Rolle eines Selektivitätsfilters in PKSs.

3. Die detaillierte Analyse der AT-vermittelten Reaktionsfolge zeigt, dass die Substratselektivität grundsätzlich in beiden Schritten der ACP-Beladung erfolgen kann. Die Beladung mit dem korrekten Substrat ist nicht über eine hydrolytische Korrekturfunktion sichergestellt, wie es beispielsweise für Aminoacyl-tRNA-Synthetasen berichtet wird. [13]

Darüber hinaus gibt eine Mutationsstudie weitere hilfreiche Aufschlüsse über das Zusammenspiel von AT und ACP. Als Testsystem dient Modul 5 (M5) der wohl bestuntersuchten PKS, 6Desoxyerythronolid B-Synthase (DEBS) (im Folgenden DEBSM5 genannt). Punktmutationen in der AT:ACP-Schnittstelle haben komplexe Effekte auf die AT-vermittelten Reaktionen. Die Sensitivität gegenüber Punktmutationen ist auf eine hohe Plastizität der AT-ACP-Interaktion zurückzuführen, wie sie auch vor kurzem für eine Typ II-FAS beschrieben wurde. [14]

Diese detaillierten Erkenntnisse über die kinetischen Eigenschaften von ATs sind höchst relevant für Engineering-Ansätze. Aus dem Satz analysierter Domänen weist besonders die AT der Säuger-FAS Eigenschaften eines primordialen Enzyms auf (schnell, polyspezifisch, plastisch) und kann vermutlich leichter neue oder veränderte Funktionen einführen als spezialisierte ATs. 
Somit scheint diese Domäne für Engineering-Ansätze gut geeignet zu sein, in denen neue oder nicht-natürliche Substrate über die AT geladen werden sollen. In anderen Ansätzen, die auf den gezielten Einbau eines spezifischen Substrats abzielen, eignet sich besonders die Verwendung von ATs der modularen PKSs für einen Domänenaustausch. Mit ihren intrinsisch strikten Substratspezifitäten können sie leicht einen hochselektiven Substrateinbau gewährleisten.

Aufbauend auf diesen Ergebnissen beschäftigt sich die vorliegende Thesis mit dem Engineering der Säuger-FAS zu PKS-ähnlichen Fertigungsstraßen mit dem Ziel, maßgeschneiderte Produkte effizient herzustellen. FASs sind grundsätzlich deutlich schneller und effizienter in ihrer Produktsynthese als PKSs und deshalb vielversprechende Kandidaten. ${ }^{[15,16]}$ Jahrelange Erfahrung im Bereich der FAS-Charakterisierung und des FAS-Engineerings fließt in dieses ambitionierte Projekt mit ein und beleuchtet besonders die Säuger-FAS als geeignetes System. Die offene Architektur bietet die Möglichkeit, Domänen zu entfernen, ${ }^{[17]}$ was Vorausetzung für nicht- und partiell-reduzierende FAS-Module ist. Folgende Engineering-Aspekte müssen beachtet werden, um die iterative Säuger-FAS in eine vektorielle Synthesestraße umzuwandeln:

1. In der Säuger-FAS gibt es nur eine AT, die für die Beladung mit Starter- und Elongationssubstrat zuständig ist. In der FAS-basierten Fertigungsstraße muss diese Beladungsfunktion von Starter- und Elongationssubstrat getrennt werden. Es muss sichergestellt werden, dass jedes Modul über die eigene AT gezielt ein Substrat einbaut und gegen das oder die anderen Substrate selektiert.

2. Die Prozessierung der Substrate innerhalb der FAS-Module (Kettenelongation) muss sichergestellt werden. Es müssen verschiedene FAS-Module entworfen werden, die über ihre Domänenzusammensetzung den Erhalt der verschiedenen funktionellen Gruppen ermöglichen. Für maximale Variabilität müssen vier FAS-Module erstellt werden.

3. Die Interaktion und Kommunikation zwischen den Modulen (Kettentranslokation) müssen sichergestellt werden. In PKSs kommen hierfür kovalente und nicht-kovalente Linker zum Einsatz. Geeignete Linker müssen für die FAS-Fertigungsstraße entwickelt werden.

Die vorliegende Thesis widmet sich vor allem dem ersten Aspekt, der Installation einer spezifischen Ladefunktion. Hierzu gibt es prinzipiell zwei Möglichkeiten: [18]

1. Zum einen bietet gezielte Mutagenese die Möglichkeit, die Substratspezifität der AT zu beeinflussen. Diese Herangehensweise erzielt zwar eine veränderte, aber oftmals keine strikte Substratspezifität. Mutagenese der AT der Säuger-FAS identifizierte die Mutation R606A, die eine drastische Verschiebung von der Beladung mit Elongationssubstrat zur Beladung mit Startersubstrat hervorrief. ${ }^{[9,19]}$ Diese punktmutierte AT-Domäne kann für ein FAS-basiertes Startermodul interessant sein. 
2. Wie bereits erwähnt, kann durch den Domänenaustausch mit einer intrinsisch spezifischen AT eine selektive Ladefunktion in ein unspezifisches System eingeführt werden. Diese Vorgehensweise stellt zwar grundsätzlich eine hohe Spezifität in Aussicht, ist aber oft mit starken Stabilitäts- und Aktivitätseinbußen aufgrund von künstlichen permanenten und nicht-permanenten Protein-Protein-Interaktionen verbunden.

Die vorliegende Thesis setzt den Fokus auf die Installation einer spezifischen Ladefunktion von Elongationssubstraten in der Säuger-FAS mittels Domänenaustausch. Hierzu werden substratspezifische ATs von modularen PKSs verwendet. Erste Experimente in der Volllängen-FAS zeigen, dass der Domänenaustausch mit deutlichen Expressionsausbeute- und Stabilitätsverlusten einhergeht. Um zunächst stabile FAS/PKS-Chimären zu etablieren, wird die FAS auf das Subkonstrukt KS-AT reduziert. Nach erfolgreicher Installation der Ladefunktion in der Didomäne soll die stabile und aktive Chimäre in ein FAS-Elongationsmodul implementiert werden.

Zunächst liegt der Fokus auf der Erstellung einer FAS/DEBSM5-Chimäre. Struktur- und Sequenzalignments werden herangezogen, um geeignete Domänenschnittstellen zu finden. Diverse Grenzen werden experimentell getestet - inklusive in der Literatur für modulare PKSs beschriebene universelle Schnittstellen. ${ }^{20]}$ Alle acht Chimären zeigen deutliche Ausbeute- und Stabilitätseinbußen und auch die in anderen Systemen etablierten Grenzen zeigen keine Verbesserung. Dies wirft die folgenden Fragen auf: Wurden die passenden Domänengrenzen noch nicht gefunden? Sind die beiden Proteine überhaupt kompatibel für den AT-Austausch? Sind die beiden Proteine grundsätzlich geeignet für einen AT-Austausch?

Um diese Fragen zu beantworten, werden die beiden Wildtyp-Proteine in ihrer Robustheit gegenüber Mutationen in den Domänenschnittstellen untersucht. Hierzu werden verschiedene Bioinformatikprogramme verwendet, um für die Proteinstabilität relevante Aminosäuren zu identifizieren und die Effekte einer Mutation dieser Reste vorherzusagen. Die Säuger-FASDidomäne stellt sich hierbei als äußerst robust heraus und erlaubt die Mutation vieler Reste in den Schnittstellen. Allerdings identifizieren In silico-Vorhersage und experimentelle Daten eine Aminosäure, die für die Stabilität der Didomäne essentiell zu sein scheint. Grundsätzlich scheint sich die Säuger-FAS unter Bedacht des gefundenen Hotspots sehr gut für einen AT-Austausch zu eignen. DEBSM5 hingegen zeigt keinerlei Toleranz gegenüber Punktmutationen in den Schnittstellen und jede Mutation führt experimentell zu drastischen Stabilitätseinbrüchen, die in diesem Ausmaß nicht für alle Reste von den Bioinformatikprogrammen vorhergesagt werden. Dies kann auf die verhältnismäßig schlechte Qualität der Strukturlösung zurückzuführen sein, da diese die Grundlage aller Vorhersagen darstellt. Die Studie zeigt deutlich, dass DEBSM5 nicht für einen AT-Austausch geeignet und somit höchstwahrscheinlich für die Instabilität der FAS/DEBSM5-Chimären verantwortlich ist. Eine weitere Analyse der Schnittstellen zeigt zudem, dass die beiden Proteine nicht kompatibel für einen AT-Austausch sind. 
Da die Säuger-FAS mit ihrer Robustheit und Plastizität an sich aber äußerst gut für diesen Engineering-Ansatz geeignet ist, wird in weiteren Experimenten ihre AT durch Elongationssubstrat-spezifische ATs aus Escherichia coli (E. coli) FAS und DEBSM6 ersetzt. Auch hier zeigen sich teilweise Stabilitätsprobleme, die ebenfalls auf Inkompatibilitäten der Systeme zurückzuführen sind. Eine der Säuger-FAS/DEBSM6-Chimären zeigt jedoch in ersten Untersuchungen hohe Stabilität und Qualität. Die weitere Charakterisierung der AT-Domäne lässt erkennen, dass die Kinetiken in der Chimäre im Vergleich zum Wildtyp-DEBSM6 zwar verändert sind, aber die Chimäre immer noch $70 \%$ der Wildtyp-Protein-Umsatzrate aufweist. Dies stellt den ersten Meilenstein in der Etablierung der chimären Fertigungsstraße dar: die Etablierung geeigneter Domänengrenzen für den AT-Austausch in der Säuger-FAS. Leider ist die DEBSM6 AT an sich sehr langsam und deshalb nur bedingt für den Einsatz in der FASbasierten Fertigungsstraße geeignet. Nun müssen geeignete Donor-ATs gefunden werden.

Darauf aufbauend werden im Minimalmodul der Säuger-FAS (KS-AT-ACP) andere spezifische ATs von verschiedenen PKSs ohne vorherige Experimente mit denselben Domänengrenzen eingebaut. Nur eines der neun Konstrukte zeigt akzeptable Expressionsausbeuten und wird weiter analysiert. Experimente zeigen, dass dieses FAS-basierte Elongationsmodul tatsächlich nicht mehr Startersubstrat, sondern nur noch Elongationssubstrat lädt. Dies demonstriert das Potential des Engineering-Ansatzes und dessen grundsätzliche Machbarkeit. Im Zusammenspiel mit einem PKS-Startermodul werden jedoch leider nur geringe Mengen des Produkts detektiert. Dies kann auf alle drei oben genannten Engineering-Aspekte zurückzuführen sein. Um ein intrinsisches Problem der Wildtyp-AT oder ein Problem in der chimären Ladefunktion ausschließen zu können, ist die Analyse des Subkonstrukts KS-AT der Wildtyp-PKS und der Chimäre nötig. Falls die Chimäre AT-Aktivität zeigt, muss das Design des Elongationsmoduls überdacht und die intermodulare Kommunikation innerhalb der Fertigungsstraße optimiert werden.

Insgesamt trägt die vorliegende Thesis maßgeblich zum detaillierten mechanistischen Verständnis der AT in FASs und PKSs bei. Sie beleuchtet Unterschiede der verschiedenen Klassen und Typen und bietet Einblicke in die essentielle AT-ACP-Interaktion einer PKS. Darüber hinaus werden erste wichtige Schritte zur Etablierung einer chimären Fertigungsstraße unternommen. Die Ergebnisse des Protein-Engineerings zeigen, dass die Säuger-FAS für die Etablierung einer Synthesestraße prädestiniert ist. Geeignete Domänenschnittstellen für einen AT-Austausch werden identifiziert und erste aktive Chimären erfolgreich erstellt. Weiterhin zeigt sich, dass sich jedes System individuell verhält und minimale Änderungen in den Grenzflächen einen großen Einfluss auf Stabilität und Aktivität haben können. Zukünftige Engineering-Ansätze sollten auf einer Analyse der KS-AT-Schnittstellen des Wildtyp-Proteins - unterstützt durch den Einsatz von Bioinformatikprogrammen - und der Charakterisierung der Donor-AT im Wildtyp- und im chimären Subkonstrukt KS-AT aufbauen, um eine spezifische Ladefunktion in FAS-basierten Modulen erfolgreich zu installieren. 


\section{Contents}

List of Tables . . . . . . . . . . . . . . . . . . . . . . . . . VII

List of Figures . . . . . . . . . . . . . . . . . . . . . . . . . . VIII

List of Abbreviations . . . . . . . . . . . . . . . . . . . . . X

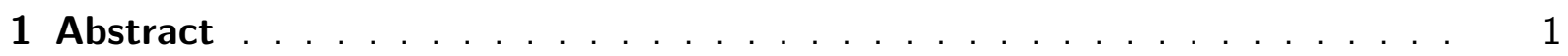

2 Introduction . . . . . . . . . . . . . . . . . . . . 3

2.1 Natural Products in Modern Medicine . . . . . . . . . . . . . . . . . 3

2.2 Fatty Acid and Polyketide Synthases . . . . . . . . . . . . . . . . . . . . . . 4

2.3 Biosynthesis of Fatty Acids and Polyketides . . . . . . . . . . . . . . . . . . . . . 5

2.4 Evolution of Fatty Acid and Polyketide Synthases . . . . . . . . . . . . . . . . 8

2.5 Structural Comparison of Fatty Acid and Polyketide Synthases . . . . . . . . . . 10

2.6 Key Players in Fatty Acid and Polyketide Synthesis . . . . . . . . . . . . . . . . 13

2.6.1 The Acyl Carrier Protein . . . . . . . . . . . . . . . . . . . . . . . . . 13

2.6.2 The Acyl Transferase . . . . . . . . . . . . . . . . . . . . . . . . . . . . 14

2.6.3 The $\beta$-Ketoacyl Synthase . . . . . . . . . . . . . . . . . . . . . 17

2.6.4 Important Domain-Domain Interactions . . . . . . . . . . . . . . . . 20

2.7 Generation of New Natural Products . . . . . . . . . . . . . . . . . . . . . . . 21

2.8 Motivation Behind This Thesis . . . . . . . . . . . . . . . . . . . . . . 22

2.9 Aim . . . . . . . . . . . . . . . . . . . . . . . . . . . . . . . . . . 24

3 Results . . . . . . . . . . . . . . . . . . . . . . . . . . 25

3.1 Preliminary Work . . . . . . . . . . . . . . . . . . . . . . . . . 25

3.2 Kinetic Description of AT-mediated Reactions . . . . . . . . . . . . . . . . 25

3.2 .1 Transacylation . . . . . . . . . . . . . . . . . . . . 26

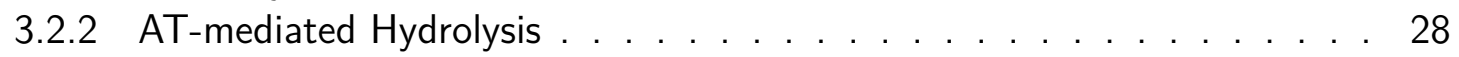

3.3 Kinetic Analysis of Acyl Transferases . . . . . . . . . . . . . . . . . . . . . . . . . 30

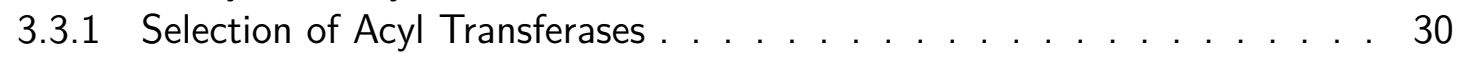

3.3.2 Isolation of Acyl Transferases . . . . . . . . . . . . . . . . . . . . . . . . . . . . . . . . . . . . . . . . . . . . .

3.3.3 Isolation of Acyl Carrier Proteins . . . . . . . . . . . . . . . . . . . . . 32

3.3.4 Initial Substrate Screening . . . . . . . . . . . . . . . . . . . 33

3.3.5 AT-mediated Hydrolysis . . . . . . . . . . . . . . . . . . . . . . . . . . . . . . . . . . . . . . . . . . . 35

3.3.6 AT-mediated Transacylation . . . . . . . . . . . . . . . . . . . . 37

3.3.7 AT:ACP Interface Mutation Study . . . . . . . . . . . . . . . . . . . 41

3.4 Generation of a FAS-Based Chimeric Elongation Module . . . . . . . . . . . . 46

3.4.1 Chimeric mFAS with Swapped DEBS AT5 and RAPS AT14 . . . . . . 46

3.4.2 Generation of mFAS/DEBS3M5 KS-AT Chimeras . . . . . . . . . . . . 52

3.4.3 Analysis of mFAS KS-AT Wild Type . . . . . . . . . . . . . . . . . . . . 55

3.4.4 Analysis of DEBS3M5 KS-AT Wild Type . . . . . . . . . . . . . . . . . 62

3.4.5 Correlation of Experimental and in silico Data . . . . . . . . . . . . . . 65

3.4.6 Generation of mFAS/FabD KS-AT Chimeras . . . . . . . . . . . . . . . 71

3.4.7 Generation of mFAS/DEBS3M6 KS-AT Chimeras . . . . . . . . . . . . . . 73

3.4.8 Analysis of mFAS/DEBS3M6 KS-AT Chimeras . . . . . . . . . . . . . . 75 
4 Discussion . . . . . . . . . . . . . . . . . . . . . . . . . . . . . . . 79

4.1 AT Activity Assay . . . . . . . . . . . . . . . . . . . . . . . . . . . . . . . . . 79

4.2 Kinetic Analysis of Acyl Transferases . . . . . . . . . . . . . . . . . . . . . . . 81

4.3 Implications of Kinetic Properties on the AT Reaction Mechanism . . . . . . . . 83

4.4 Implications of Kinetic Properties on FAS/PKS Function and Evolution . . . . . 84

4.5 Impact of Interface Mutations on Transacylation Kinetics . . . . . . . . . . . . 85

4.6 Implications of Interface Mutation Study on PKS Engineering . . . . . . . . . . 87

4.7 Towards the Generation of a Functional Chimeric mFAS Module . . . . . . . . . 88

4.8 Experimental Outlook: Implementation of Chimeras in mFAS Modules . . . . . 96

4.9 Strategies for Creating Functional Chimeras . . . . . . . . . . . . . . . . . . . 99

4.10 Bioinformatics Tools in Chimeric Protein Design . . . . . . . . . . . . . . . . 102

4.11 Conclusion and Outlook . . . . . . . . . . . . . . . . . . . . 103

5 Experimental Procedures f . . . . . . . . . . . . . . . . . . 106

5.1 Material and Methods . . . . . . . . . . . . . . . . . . . . . . . 106

5.1 .1 Molecular Cloning . . . . . . . . . . . . . . . . . . . . . . . . . 106

5.1 .2 Protein Expression . . . . . . . . . . . . . . . . . . . . . 107

5.1.3 Purification of AT Constructs and mFAS Modules . . . . . . . . . . . . 107

5.1 .4 Purification of ACPs . . . . . . . . . . . . . . . . . . . . . . . . . 108

5.1 .5 Protein Concentration . . . . . . . . . . . . . . . . . . . . . . . . 108

5.1 .6 SDS-PAGE Analysis . . . . . . . . . . . . . . . . . . . . . . . . . . 111

5.1 .7 Mass Spectrometric Analysis of ACPs . . . . . . . . . . . . . . . . . . . . . . . . . . 112

5.1 .8 CoA-488 Assay . . . . . . . . . . . . . . . . . . . . . . . . . . . 1112

5.1 .9 HPLC-SEC Analysis . . . . . . . . . . . . . . . . . . . . . . . . . 112

5.1 .10 Thermal Shift Assay . . . . . . . . . . . . . . . . . . . . . . . 113

5.1.11 AT Activity Assay . . . . . . . . . . . . . . . . . . . . . . . . . . . . . . . . 113

5.1 .12 TAL Assay . . . . . . . . . . . . . . . . . . . . . . . . . . 114

5.1.13 Design of DEBS3M5 AT:ACP Interface Mutations . . . . . . . . . . . 115

5.1 .14 Bioinformatics Tools . . . . . . . . . . . . . . . . . . . . . . 115

5.2 Synthesis of 2-Methylbutyryl-CoA . . . . . . . . . . . . . . . . . 117

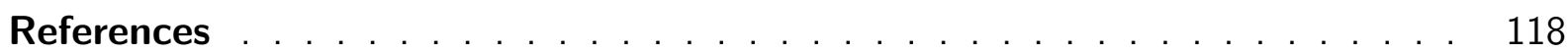

Appendix . . . . . . . . . . . . . . . . . . . . . . . . . . 140

Statement of Personal Contributions . . . . . . . . . . . . . . . . . . . 206

Eidesstattliche Erklärung . . . . . . . . . . . . . . . . . . . . . . . . . . . . . . 212

Lebenslauf . . . . . . . . . . . . . . . . . . . . . . . . . . . . . . . . . . 213

Danksagung . . . . . . . . . . . . . . . . . . . . . . . . . . . . . . . . 214 


\section{List of Tables}

3.1 Hydrolysis parameters of FAS and PKS ATs. . . . . . . . . . . . . . . . . 36

3.2 Transacylation parameters of FAS and PKS ATs. . . . . . . . . . . . . . . . . 38

3.3 Kinetic parameters of DEBS M5 AT:ACP interface mutants. . . . . . . . . . . 43

3.4 Expression yields of chimeric mFAS with DEBS AT5 and RAPS AT14. . . . . . 50

3.5 Expression yields of mFAS/DEBS M5 KS-AT chimeras. . . . . . . . . . . . . . 54

3.6 Results of bioinformatic analysis of mFAS KS-AT. . . . . . . . . . . . . . . . 57

3.7 Expression yields of mFAS KS-AT mutants. . . . . . . . . . . . . . . . . 58

3.8 Results of bioinformatic analysis of DEBS M5 KS-AT . . . . . . . . . . . . 63

3.9 Expression yields of DEBS M5 KS-AT mutants. . . . . . . . . . . . . . . . . . 64

3.10 Expression yields of mFAS/FabD KS-AT chimeras. . . . . . . . . . . . . . . . 71

3.11 Expression yields of mFAS/DEBS M6 KS-AT chimeras. . . . . . . . . . . . 73

3.12 Average expression yields of mFAS/DEBS M6 KS-AT chimeras. . . . . . . . . . 75

3.13 Average expression yields of mFAS and DEBS M6 ACPs. . . . . . . . . . . . . 75

3.14 Transacylation parameters of DEBS M6 wild type and chimera. . . . . . . . . 78

5.1 Absorbance of wild type proteins. . . . . . . . . . . . . . . . . . . 109

5.2 Absorbance of DEBS M5 AT interface mutants. . . . . . . . . . . . . . . . . 109

5.3 Absorbance of mFAS KS-AT mutants. . . . . . . . . . . . . . . . . . . . . 110

5.4 Absorbance of DEBS M5 KS-AT mutants. . . . . . . . . . . . . . . . . . 110

5.5 Absorbance of chimeras. . . . . . . . . . . . . . . . . . . . . . . . 111

S1 Expression yields of FAS and PKS ATs. . . . . . . . . . . . . . . . . . . . . 164

S2 TSA of FAS and PKS ATs and ACPs. . . . . . . . . . . . . . . . . . . . . 164

S3 Expression yields of FAS and PKS ACPs. . . . . . . . . . . . . . . . 165

S4 Mass spectrometric analysis of FAS and PKS ACPs. . . . . . . . . . . . . . 166

S5 Initial substrate screening of FAS and PKS ATs. . . . . . . . . . . . . . . . 167

S6 Hydrolysis parameters of FAS and PKS ATs after transacylation. . . . . . . . . 168

S7 Expression yields of DEBS M5 AT:ACP interface mutants. . . . . . . . . . . . 169

S8 TSA of DEBS M5 AT:ACP interface mutants. . . . . . . . . . . . . . . . . 169

S9 Mass spectrometric analysis of mFAS and DEBS M6 ACPs. . . . . . . . . . . . 170

S10 Transacylation parameters of DEBS M6 wild type and chimera (replicates). . . 171

S11 NADH calibration factor over time. . . . . . . . . . . . . . . . . . . . 172 


\section{List of Figures}

2.1 Scheme of the different FAS types. . . . . . . . . . . . . . . . . 4

2.2 Scheme of the different typel PKSs. . . . . . . . . . . . . . . 5

2.3 Biosynthesis of FASs and PKSs. . . . . . . . . . . . . . . . . . 6

2.4 Phylogenetic relationship between FASs and PKSs. . . . . . . . . . . . . . . . . . . . 8

2.5 Structure and domain organization of type I FAS systems. . . . . . . . . . . . 11

2.6 Structural models for modular PKSs. . . . . . . . . . . . . . . . . . . . . . . 12

2.7 Structure of the ACP and AT . . . . . . . . . . . . . . . . . . . . 14

2.8 Reaction catalyzed by the AT. . . . . . . . . . . . . . . . . . . . . 15

2.9 Reaction catalyzed by the KS . . . . . . . . . . . . . . . . . . . . . 19

2.10 Idea of the FAS-based assembly line. . . . . . . . . . . . . . . . . . 23

3.1 Scheme of AT-mediated reactions. . . . . . . . . . . . . . . . . . . 25

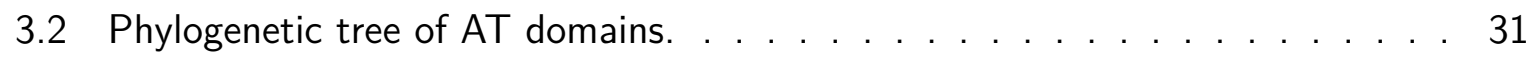

3.3 Sequence alignment of AT domains. . . . . . . . . . . . . . . . . . . . . . 31

3.4 Transacylation and hydrolysis screening of FAS and PKS ATs. . . . . . . . . . . 34

3.5 Transacylation parameters of FAS and PKS ATs. . . . . . . . . . . . . . . . . . 40

3.6 Modeled DEBS M5 AT:ACP interface. . . . . . . . . . . . . . . . . 41

3.7 Transacylation and hydrolysis screening of DEBS M5 AT:ACP interface mutants. 42

3.8 Transacylation parameters of DEBS M5 AT:ACP interface mutants. . . . . . . 44

3.9 Transacylation transition state energy of DEBS M5 AT:ACP interface mutants. 45

3.10 Structural similarity of mFAS and DEBS M5 KS-AT. . . . . . . . . . . . . . . 47

3.11 Domain boundaries for mFAS/DEBS AT5 and RAPS AT14 chimeras. . . . . . 49

3.12 Analysis of chimeric mFAS with DEBS AT5 and RAPS AT14. . . . . . . . . . . 51

3.13 Design of mFAS/DEBS M5 KS-AT chimeras. . . . . . . . . . . . . . . . . . . 53

3.14 Analysis of mFAS/DEBS M5 KS-AT chimeras. . . . . . . . . . . . . . . . 55

3.15 Mutation sites in mFAS KS-AT. . . . . . . . . . . . . . . . . 56

3.16 SDS-PAGE analysis of mFAS KS-AT mutants. . . . . . . . . . . . . . . . 59

3.17 TSA of mFAS KS-AT mutants. . . . . . . . . . . . . . . . . . . . . . . . . 59

3.18 HPLC-SEC of mFAS KS-AT mutants. . . . . . . . . . . . . . . . . . 60

3.19 Interfacial salt bridge in mFAS KS-AT. . . . . . . . . . . . . . . . 61

3.20 Mutation sites in DEBS M5 KS-AT . . . . . . . . . . . . . . . . . . . 62

3.21 Analysis of DEBS M5 KS-AT mutants. . . . . . . . . . . . . . . . . . . 64

3.22 Correlation of Rosetta predictions with mFAS KS-AT yields. . . . . . . . . . . 66

3.23 Correlation of Rosetta predictions with mFAS KS-AT melting temperatures. . . 67

3.24 Correlation of Rosetta predictions with mFAS KS-AT oligomeric states. . . . . 68

3.25 Correlation of Rosetta predictions with DEBS M5 KS-AT yields. . . . . . . . . 70

3.26 Domain boundaries for mFAS/FabD KS-AT chimeras. . . . . . . . . . . . . . 71

3.27 Analysis of mFAS/FabD KS-AT chimeras. . . . . . . . . . . . . . . . . . . 72

3.28 Domain boundaries for mFAS/DEBS AT6 KS-AT chimeras. . . . . . . . . . . 73

3.29 Analysis of mFAS/DEBS M6 chimeric KS-AT. . . . . . . . . . . . . . . . . . 74

3.30 Analysis of mFAS/DEBS M6 KS-AT chimeras and wild type DEBS M6 KS-AT. 76

3.31 Transacylation screening of mFAS/DEBS M6 KS-AT chimeras. . . . . . . . . 77 
3.32 Transacylation curves of DEBS M6 KS-AT wild type and chimera. . . . . . . . 78

4.1 Plasticity of transacylation kinetics of DEBS M5 AT:ACP interface mutants. . 86

4.2 Contact potential of mFAS KS-AT wild type. . . . . . . . . . . . . . . . 93

4.3 Contact potential of DEBS M5 KS-AT wild type. . . . . . . . . . . . . . . . . 94

4.4 Structure and contact potential of FabD . . . . . . . . . . . . . . . . . . . . 95

4.5 Idea of assembly line with chimeric elongation module. . . . . . . . . . . . 97

S1 SDS-PAGEs of FAS and PKS ATs. . . . . . . . . . . . . . . . . . 173

S2 Size exclusion chromatograms of FAS and PKS ATs. . . . . . . . . . . . . . . 175

S3 TSA melting curves of FAS and PKS ATs. . . . . . . . . . . . . . . . . . . 177

S4 Analytical SDS-PAGEs of FAS and PKS ACPs. . . . . . . . . . . . . . . . . . 177

S5 Mass spectrometric analysis of FAS and PKS ACPs. . . . . . . . . . . . . . . . . 179

S6 TSA melting curves of FAS and PKS ACPs. . . . . . . . . . . . . . . . . . . 182

S7 Titration curves for AT-mediated hydrolysis. . . . . . . . . . . . . . . . . . . . . 184

S8 Titration curves for AT-mediated transacylation. . . . . . . . . . . . . . . . 187

S9 Detailed model of DEBS M5 AT:ACP interface. . . . . . . . . . . . . . . . 188

S10 SDS-PAGEs of DEBS M5 AT:ACP interface mutants. . . . . . . . . . . . . . . . 189

S11 Size exclusion chromatograms of DEBS M5 AT:ACP interface mutants. . . . . 191

S12 TSA melting curves of DEBS M5 AT:ACP interface mutants. . . . . . . . . . 193

S13 Hydrolysis curves for DEBS M5 AT:ACP interface mutants. . . . . . . . . . . 194

S14 Transacylation curves for DEBS M5 AT:ACP interface mutants. . . . . . . . 195

S15 ACP titration curve of DEBS M5 AT:ACP mutant R850E. . . . . . . . . . . 196

S16 Purification of chimeric mFAS with DEBS AT5. . . . . . . . . . . . . . . 197

S17 Correlation of bioinformatics tools predictions with mFAS KS-AT yields. . . . . 198

S18 TSA melting curves of mFAS/DEBS M6 KS-AT chimeras. . . . . . . . . . . . . . 199

S19 Mass spectrometric analysis of mFAS and DEBS M6 ACPs. . . . . . . . . . . 200

S20 Transacylation curves for mFAS/DEBS M6 KS-AT chimera (replicates). . . . . 201

S21 Transacylation curves for DEBS M6 KS-AT (replicates). . . . . . . . . . . . . 202

S22 Electron density map of DEBS M5 AT residue R850 . . . . . . . . . . . . . . 203

S23 NADH calibration. . . . . . . . . . . . . . . . . . . . . . . . . . . . . . . . . . . . . . . . . . . . . . . . .

S24 NMR of 2-methybutyryl-CoA . . . . . . . . . . . . . . . . . . 205 


\section{List of Abbreviations}

\begin{tabular}{|c|c|}
\hline 2-MB & 2-methylbutyryl \\
\hline Ac & acetyl \\
\hline ACP & acyl carrier protein \\
\hline AT & acyl transferase \\
\hline AVES & avermectin polyketide synthase \\
\hline BSA & bovine serum albumin \\
\hline CMN-bacteria & Corynebacteria, Mycobacteria and Nocardia \\
\hline $\mathrm{CoA}$ & coenzyme $A$ \\
\hline DEBS & 6-deoxyerythronolide B synthase \\
\hline DD & docking domain \\
\hline $\mathrm{DH}$ & dehydratase \\
\hline DNA & deoxyribonucleic acid \\
\hline DNase & deoxyribonuclease \\
\hline DTT & 1,4-dithiothreitol \\
\hline E. coli & Escherichia coli \\
\hline EcPKS & Elysia chlorotica polyketide synthase \\
\hline EDTA & ethylendiamintetraacetic acid \\
\hline EM & electron microscopy \\
\hline ER & $\beta$-enoyl reductase \\
\hline FAS & fatty acid synthase \\
\hline GMQE & Global model quality estimation \\
\hline HPLC & high performance liquid chromatography \\
\hline IPTG & isopropyl $\beta$-D-1-thiogalactopyranoside \\
\hline $\mathrm{K}_{\mathrm{M}}$ & Michaelis-Menten constant \\
\hline KAL & KS-AT linker \\
\hline $\mathrm{KR}$ & $\beta$-ketoacyl reductase \\
\hline KS & $\beta$-ketoacyl synthase \\
\hline LB & liquid broth \\
\hline LD & linker domain \\
\hline Mal & malonyl \\
\hline MAS & mycocerosic acid synthase \\
\hline MAT & malonyl/acetyl transferase \\
\hline MBP & maltose binding protein \\
\hline mFAS & murine FAS \\
\hline MMal & methylmalonyl \\
\hline MS & mass spectrometry \\
\hline
\end{tabular}


MSAS

$M \times$

NAD

NADPH

NMR

Npt

NTA

OD

PAL

PCR

PikA

PKS

PPT

Prop

QMEAN

QM/MM

RAPS

REU

RFU

RSRZ

SAXS

SDS-PAGE

SEC

S. erythraea

Sfp

SOC

SPNS

TAL

TB

TE

TPP

TSA 6-methylsalicylic acid synthase

module $x$

nicotinamide adenine dinucleotide

nicotinamide adenine dinucleotide phosphate

nuclear magnetic resonance

4'-phosphopantetheinyl transferase

nitrilotriacetic acid

optical density

post-AT linker

polymerase chain reaction

pikromycin synthase

polyketide synthase

phosphopantetheinyl transferase

propionyl

quality model energy analysis

quantum mechanics/molecular mechanics

rapamycin synthase

Rosetta energy unit

relative fluorescence unit

real-space R-value Z-score

small-angle $\mathrm{X}$-ray scattering

sodium dodecyl sulfate polyacrylamide gel electrophoresis

size exclusion chromatography

Saccharopolyspora erythraea

4'-phosphopantetheinyl transferase

super optimal broth with catabolite repression

spinosyn synthase

triacetic acid lactone

terrific broth

thioesterase

thiamine pyrophosphate

thermal shift assay 


\section{Abstract}

Polyketides are highly valuable natural products, which are widely used as pharmaceuticals due to their beneficial characteristics, comprising antibacterial, antifungal, immunosuppressive, and antitumor properties, among others. [3] Their biosynthesis is performed by large and complex multiproteins, the polyketide synthases (PKSs). ${ }^{[4]}$ This study solely focuses on the class of type I PKSs, which arrange all their enzymatic domains on one ore more polypeptides. Despite their high medical value, little is known about mechanistic details in PKSs.

One central domain is the acyl transferase (AT), which is present in all PKSs and channels small acyl substrates into the enzyme. More precisely, the AT loads the substrates onto the essential acyl carrier protein (ACP), which subsequently shuttles the substrates and all intermediates for condensation and modification to additional domains to build the final polyketide.

Some PKSs use their domains several times during biosynthesis and work iteratively - these are called iterative PKSs. Others feature several sets of domains, each being used only once during biosynthesis - these PKSs are called modular PKSs. All PKSs or PKS modules consist of minimum three essential domains to connect the acyl substrates. ${ }^{[5]}$ Three modifying domains are optional and can enlarge the minimal set. According to the domain composition, the acyl substrate is fully reduced, partly reduced, or not reduced at all. This variation of modifying domains accounts for the huge structural and therefore functional variety of polyketides.

Even though the structure of fatty acids is not exactly reminiscent of polyketides, their biosynthetic pathways are closely related. Fatty acid biosynthesis is carried out by fatty acid synthases (FASs), which share many similarities with PKSs. ${ }^{[3,6]}$ Both megasynthases feature the same domains, performing the same reactions to connect and modify small acyl substrates. In contrast to PKSs, FASs always contain one full set of modifying domains which is used iteratively, leading to fully reduced fatty acids.

The present thesis extensively analyzes the AT of different PKSs in its substrate selectivity, ATACP domain-domain interaction, and enzymatic kinetic properties. The following key findings are revealed through comparison: 1.) ATs of PKSs appear slower than the ones of FASs, which may reflect the different scopes of biosynthetic pathways. Fatty acids as essential compounds in all organisms are needed in high amounts for physiological functions, whereas polyketides as secondary metabolites only require basal concentrations to take effect. 2.) The slower ATs from modular PKSs do not load non-native substrates even in absence of the native substrates. This is different to the faster ATs from iterative PKSs and FASs, which indicates high substrate 


\section{Abstract}

specificity solely for the ATs from modular PKSs and emphasizes their role as gatekeepers in polyketide synthesis. 3.) The substrate selectivity can emerge in either the first or the second step of the AT-mediated ACP loading and is not assured by a hydrolytic proofreading function.

Moreover, a mutational study on the AT-ACP interaction in the modular PKS 6-deoxyerythronolide $B$ synthase (DEBS) shows that single surface point mutations can influence AT-mediated reactions in a complex manner. Data reveals high enzyme kinetic plasticity of the AT-ACP interaction, which was also recently demonstrated for the interaction in a type II FAS. [14]

Based on these findings, the mammalian FAS is engineered towards a modular PKS-like assembly line with the long-term goal to rationally synthesize new products. Basically, three important aspects need to be considered: 1.) AT's loading needs to be splitted in specific loading of a priming substrate by a priming AT and in specific loading of an elongation substrate by an elongation AT. 2.) FAS-based elongation modules need to be designed with varying domain compositions for introducing functional groups in the product. 3.) Covalent and non-covalent linkers need to be designed for connection of priming and elongation modules.

This study focuses on the first aspect, splitting loading of priming and elongation substrates. An elongation substrate-specific AT is installed in the mammalian FAS via domain swapping. Since ATs from modular PKSs were proven to be substrate specific, these are used to exchange the mammalian FAS AT. This work demonstrates that it is extremely challenging to create stable and functional chimeras, but first essential steps are taken. Proper domain boundaries for AT swapping are established and a stable chimera with $70 \%$ wild type AT activity is created. However, this chimera is only of limited value for application in an elongation module due to the intrinsic slow turnover rate of the wild type AT. Using another PKS AT, a stable elongation module is designed and analyzed in its activity in combination with a priming module. These experiments demonstrate that the loading of priming substrates are successfully suppressed in the elongation module, but nonetheless only minor turnover rates are detected in the assembly line. This can have various causes: the AT domain itself, intra-, or intermodular communication problems. Further experiments on subconstructs, including characterization of the wild type, are needed to identify the real problem.

Overall, the present thesis contributes significantly to the mechanistic understanding of AT domains in FASs and PKSs. Furthermore, although challenging, it is possible to create stable and functional chimeras via AT swapping to generate mammalian FAS-based elongation modules as shown in this thesis. However, each system behaves individually and small adaptations in domain boundaries can have a huge impact on stability and activity. Further engineering approaches should be based on detailed analysis of wild type donor ATs to be successful. 


\section{Introduction}

\subsection{Natural Products in Modern Medicine}

Natural products are highly valuable compounds being key players in the treatment of human diseases. ${ }^{21]}$ Almost two thirds of all pharmaceuticals used today involve natural products. [2] Many drugs are natural products themselves, some are derivatives created semisynthetically, others are chemically synthesized using natural products as lead structures.

Natural products show immense chemical diversity resulting in a large variety of medically relevant properties as antitumor, [22] immunosuppressive, ${ }^{23]}$ and antimalarial ${ }^{[24]}$ functions. ${ }^{[25]}$ Even though natural products are widely used for treatment of various diseases, the most relevant aspect is presumably the treatment of bacterial infections. ${ }^{[26-28]}$ Those are one of the most prevalent causes of death worldwide, including developed countries. ${ }^{[2,29]}$ The excessive use of antibiotics and other drugs has resulted in resistances, particularly in hospitals, which requires the discovery and development of new pharmaceuticals. ${ }^{[30]}$ In order to generate new natural products, it is crucial to understand their natural synthesis.

During the metabolism of living entities, thousands of chemical reactions, catalyzed by enzymes, take place. The metabolic pathway of all essential compounds - such as amino acids, lipids, sugars, fatty acids - was found to be evolutionary conserved among all organisms. $[31,32]$ However, certain species - as plants, bacteria, and fungi - evolved distinct expanded metabolic pathways for the production of natural products, adapted to the needs of the host. ${ }^{[33]}$ Despite their chemical diversity, natural products are generated from basic building blocks - acetate, mevalonate, and shikimate. During biosynthesis, these building blocks are transformed and combined in many different ways leading to numerous classes of natural products, including polyketides, steroids, and flavonoids. Polyketides are closely related to fatty acids, both being products of the acetate pathway. ${ }^{[29]}$ 


\subsection{Fatty Acid and Polyketide Synthases}

Fatty acids play a vital role in living organisms. They fulfill a variety of important functions as central building blocks of biological membranes and major energy source. ${ }^{[11,34]}$ They act as modulator and messenger, involving positive and negative regulation of signaling cascades. ${ }^{[10,35]}$ Thus, the fatty acid biosynthesis is essential for all organisms except archaea. ${ }^{[36]}$ It is performed by fatty acid synthases (FASs), multienzymes, which consist of a certain set of

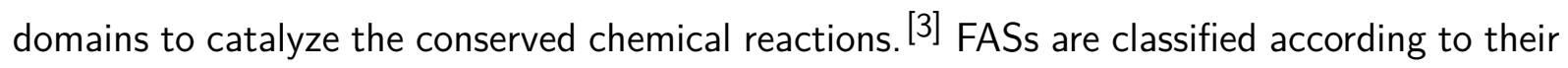
domain arrangement: Type I FASs are large multifunctional polypeptides as found in fungi, animals, and some bacteria ${ }^{i}[7,37,38]$ In contrast, type II FASs consist of dissociated monofunctional proteins as found in bacteria, plants, and eukaryotic mitochondria (Fig. 2.1). ${ }^{[39,40]}$ In both FAS types, the same set of domains catalyzes all biosynthetic reactions in an iterative manner. ${ }^{[3,41]}$

\section{Type I FAS}

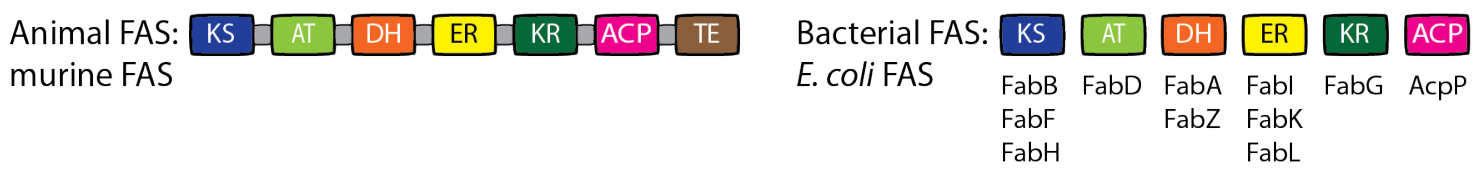

Figure 2.1: Scheme of the different FAS types. Typel FAS systems of fungi and animals are large multifunctional polypeptide chains, whereas typell FAS systems of bacteria and plants are individual monofunctional proteins. Abbreviations: $\beta$-ketoacyl synthase (KS), acyl transferase (AT), dehydratase (DH), $\beta$-enoyl reductase (ER), $\beta$-ketoacyl reductase $(K R)$, acyl carrier protein $(\mathrm{ACP})$, thioesterase (TE). Adapted from reference. ${ }^{[42]}$

The same set of domains is also found in polyketide synthases (PKSs), which are responsible for the de novo synthesis of polyketides. ${ }^{[5,43]}$ They carry out the same chemical reactions and are classified as follows: Type I PKS systems found in bacteria, fungi, and plants are multifunctional polypeptide chains similar to type I FASs, whereas type II PKSs found in prokaryotes consist of freestanding monofunctional proteins like type II FAS systems. ${ }^{[3,4]}$ Like FASs, type I PKSs of fungi contain one set of catalytic domains which is used iteratively, whereas the majority of bacterial type I PKS systems consists of multiple sets of catalytic domains, so-called modules, each being used only one time during synthesis (Fig. 2.2). ${ }^{[44]}$ These modular, assembly line-like PKSs contain either an integrated (cis) or a distinct (trans) acyl transferase (AT) domain. [45] Their modules are either covalently linked or bridged non-covalently by $\alpha$-helical linkers, the so-called docking domains (DDs). ${ }^{[46,47]}$ Besides type I and type II PKSs, there is a third type of PKSs, which are individual iterative condensing enzymes. ${ }^{3]}$ In contrast to type I and II PKSs, the polyketide intermediate and product are never directly attached to type III PKSs. ${ }^{[48]}$ In addition to these three types of PKSs, there are also hybrid systems found, including hybrid forms of type I/type II PKSs as well as type I/nonribosomal peptide synthases. ${ }^{4}$, 50$]$

\footnotetext{
ithe Corynebacterium-Mycobacterium-Nocardia (CMN) group of bacteria
} 


\section{Type I iterative PKS}

Bacterial PKS: KS AT, DH, KR ACP

MSAS

\section{Type I modular PKS}

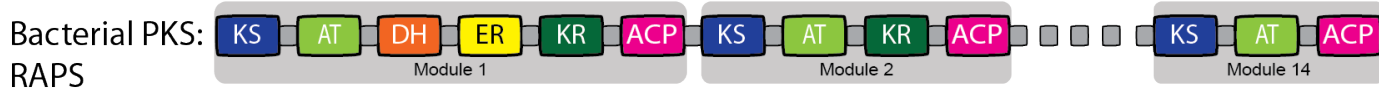

Figure 2.2: Scheme of the different typel PKSs. Type I PKS systems of fungi and some bacteria are monomodular iteratively-acting enzymes like the 6-methylsalicylic acid synthase (MSAS). The majority of bacterial type I PKSs evolved multimodular assembly line-like PKSs like the rapamycin synthase (RAPS) with either integrated (cis) or distinct (trans) AT domains. Abbreviations: $\beta$-ketoacyl synthase (KS), acyl transferase (AT), dehydratase (DH), $\beta$-enoyl reductase (ER), $\beta$-ketoacyl reductase $(K R)$, acyl carrier protein (ACP), thioesterase (TE). Adapted from reference. ${ }^{[42]}$

\subsection{Biosynthesis of Fatty Acids and Polyketides}

The megasynthases FASs and PKSs produce important products - fatty acids (most commonly palmitic (C16) and stearic acid $(\mathrm{C} 18)^{[41]}$ ) and polyketides, respectively. Whereas FASs always produce fully reduced fatty acids, polyketides show a large structural and therefore functional variety. ${ }^{[3]}$ Despite this large diversity, they are all made up of small building blocks, acyl extender units. Every FAS and PKS domain is responsible for one specific reaction, connecting and modifying these extender units.

As mentioned above, fatty acid synthesis is an iterative process and the catalytic cycle can be divided into three parts: initiation, chain elongation, and chain termination. ${ }^{[7,10,15,34,51]}$ The initial priming step is the loading of an acetyl (Ac) moiety, which is then successively elongated (Fig. 2.3A). In each round of chain elongation, a C2 unit, originated from a malonyl (Mal) moiety, is added to the growing acyl chain until the desired chain length is obtained. The acyl chain is then released either as free fatty acid or as coenzyme $A(C \circ A)$ derivative. ${ }^{[41]}$

The AT domain catalyzes the loading of acyl units of CoA esters onto the acyl carrier protein (ACP). ${ }^{[41,52,53]}$ This small protein is post-translationally modified with a phosphopantetheinyl prosthetic group, which binds acyl moieties covalently upon thioester formation. [54,55] After AT-mediated loading, the ACP shuttles the acyl substrates and intermediates to all enzymatic stations throughout the whole catalytic cycle. ${ }^{[39,56,57]}$ This central role of the ACP domain highlights the importance of domain-domain interactions during biosynthesis, ensuring specificity allowing for catalytic action on the acyl chain and simultaneously facilitating high turnover rates. ${ }^{[41]}$ 


\section{Introduction}

A

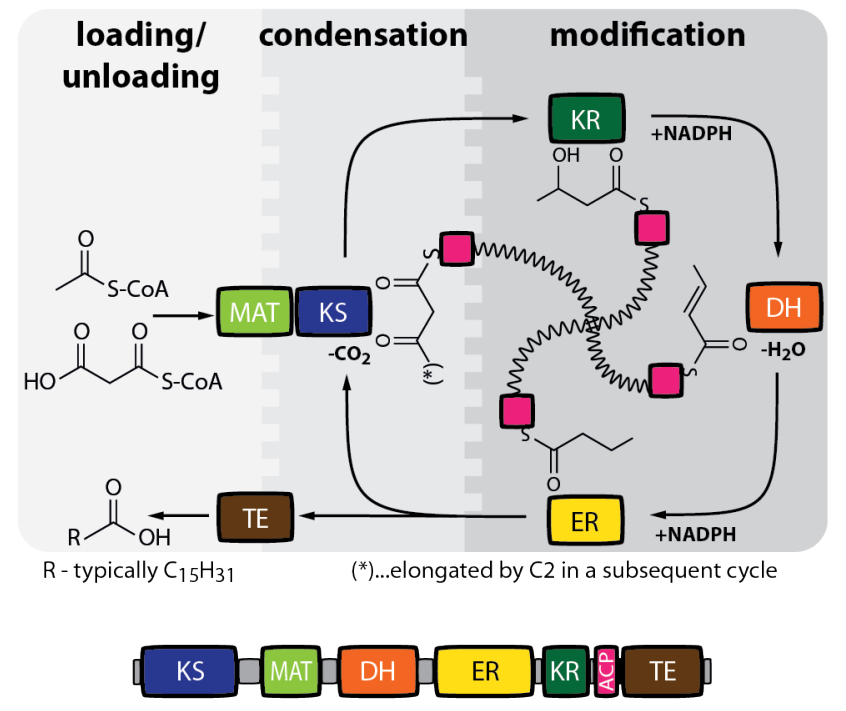

B

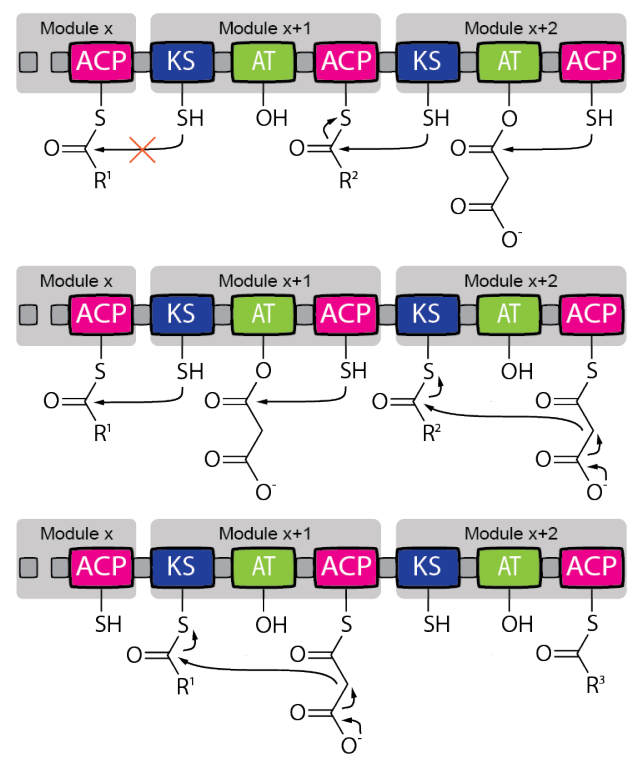

Figure 2.3: (A) Schematic cycle of fatty acid synthesis catalyzed by the mammalian FAS. Fatty acids are stepwise build from priming substrate Ac and elongation substrates Mal. After condensation, the $\beta$-ketoacyl moiety is fully reduced, before the next elongation substrate is added to the chain. After seven cycles, the final product is released by the TE domain. (B) Turnstile mechanism of modular PKSs. The KS domain cannot be acylated by the next polyketide chain, until the current product of chain elongation and subsequent modification is translocated to the downstream module (shown) or released by the TE (not shown here). Adapted from reference. ${ }^{[6]}$

The loaded substrates are connected via C-C bond formation through decarboxylative Claisen condensation, catalyzed by the $\beta$-ketoacyl synthase (KS) yielding a $\beta$-ketoacyl intermediate. ${ }^{[41,58-60]}$ Subsequently, this intermediate is successively modified by an NADPH-dependent $\beta$-ketoacyl reductase $(\mathrm{KR}),{ }^{[61-63]}$ a dehydratase $(\mathrm{DH})^{[64-66]}$ and an NADPH-dependent $\beta$ enoyl reductase $(E R)^{[67]}$ resulting in a fully saturated acyl intermediate. After several rounds of chain elongation, the product is released. In fungal and CMN-bacterial type I systems, AT mediates the transfer of the acyl moiety from ACP to CoA. ${ }^{[68]}$ Animal type I FASs and type II plant systems use a TE domain to release the free fatty acid. ${ }^{[69,70]}$ Bacterial and mitochondrial type II systems forward the acyl-ACP to other biosynthetic pathways. ${ }^{771,72]}$

Comparing FAS and PKS systems, a main difference obviously lies in the catalytic efficiency of the biosynthesis: FASs produce fatty acids in less than a second, ${ }^{[15]}$ whereas PKSs are much slower. The 6-deoxyerythronolide B synthase (DEBS) for example takes around two minutes to produce 6-deoxyerythronolide B. ${ }^{[16]}$ This difference in turnover rates might be a result of the respective function in the organisms. Fatty acids are essential for cellular processes, whereas polyketides are secondary metabolites. 


\section{Introduction}

The overall reaction series catalyzed by type I PKSs is very similar to FAS systems. ${ }^{[3]}$ Substrate loading, chain elongation, and chain termination of both megasynthases are related and all reaction intermediates remain covalently attached. The priming substrate for PKSs is typically either an Ac or propionyl (Prop) moiety and the elongation substrate is a Mal or methylmalonyl (MMal) moiety. ${ }^{[5]}$ In iterative type I PKSs, one set of catalytic domains is used repetitively like in FAS systems, whereas in modular PKSs each module contains one set of catalytic domains and is used only once, being responsible for the incorporation of one extender unit. Each set of domains in PKSs comprises three essential catalytic domains connecting the extender units: $K S, A T$, and ACP. The three domains (KR, DH, ER) responsible for further modification of the growing acyl chain are optional and can enlarge this minimal set. Whether the extender unit is fully or partly reduced or not reduced at all, depends on the specific domain composition. Based on the degree of reductive behavior, fungal type I PKSs are further divided into highly reducing (HR-), partially reducing (PR-), and non-reducing (NR-) PKSs. ${ }^{[5]}$ This diverse domain arrangement accounts for the huge structural and functional variety of compounds found within the class of PKSs. ${ }^{[73]}$ FASs, on the other hand, always use a full set of catalytic domains and, hence, can be seen as fully reducing PKSs within this nomenclature. [74]

Most modular PKSs with integrated AT domains, so-called cis-AT PKSs, follow the principle of collinearity: The modules and domains operate in the same order in which they are encoded on the DNA. ${ }^{[18]}$ This property allows for conclusions on the domain composition by analyzing the product and vice versa. Additionally to chain elongation, an intramodular process present in all FASs and PKSs, modular PKSs involve also the intermodular process of chain translocation. The ACP of one module must pass on its $\beta$-ketoacyl product to the downstream module before another extender unit can be loaded onto the ACP, which the KS domain can act on. While it remains unclear how exactly the back-transfer of one module's polyketide intermediate to its own KS is prevented, one could assume kinetic control by coupling of catalytic steps, leading to a vectorial synthesis. ${ }^{[75]}$ An alternative scenario was proposed, in which chain elongation is energetically coupled to chain translocation via a turnstile mechanism: The $\beta$-ketoacyl-ACP product of one module must be passed on to the downstream module before the KS domain can act on the next upstream $\beta$-ketoacyl-ACP product (Fig. 2.3B). [76] This mechanism avoids iterative chain elongation and is assumed to be evolutionary acquired. [6] 


\subsection{Evolution of Fatty Acid and Polyketide Synthases}

Fatty acid synthesis is an essential biosynthetic pathway among all groups of organisms and therefore likely to have evolved early. Based on the remarkable similarities between FASs and PKSs, it is reasonable to assume shared evolutionary processes. Phylogenetic analysis has revealed a long joint evolution of these two biosynthetic pathways. ${ }^{[6,42,77]}$ In the phylogenetic analysis of the highly conserved KS domain of FASs and PKSs published by $\mathrm{H}$. Jenke-Kodama and co-workers, the archaebacterial KS homologs and the bacterial KS of FabH type were used as outgroup (Fig. 2.4). [42] The FabF type KS of bacterial type II FASs was found to evolve in parallel to the KS of bacterial typell PKSs. The two typel FAS groups of fungi and CMN bacteria were found to be evolutionary close and to predate the third FASI group of animals, to which they are apparently only distantly related. As oldest typel PKSs, some bacterial iterative systems were found which evolved in parallel to glycolipid synthases. Modular type I PKSs then evolved in bacteria either with cis- or trans-AT domains in parallel to iterative type I PKSs of fungi and bacteria and the animal type I FASs. [42]

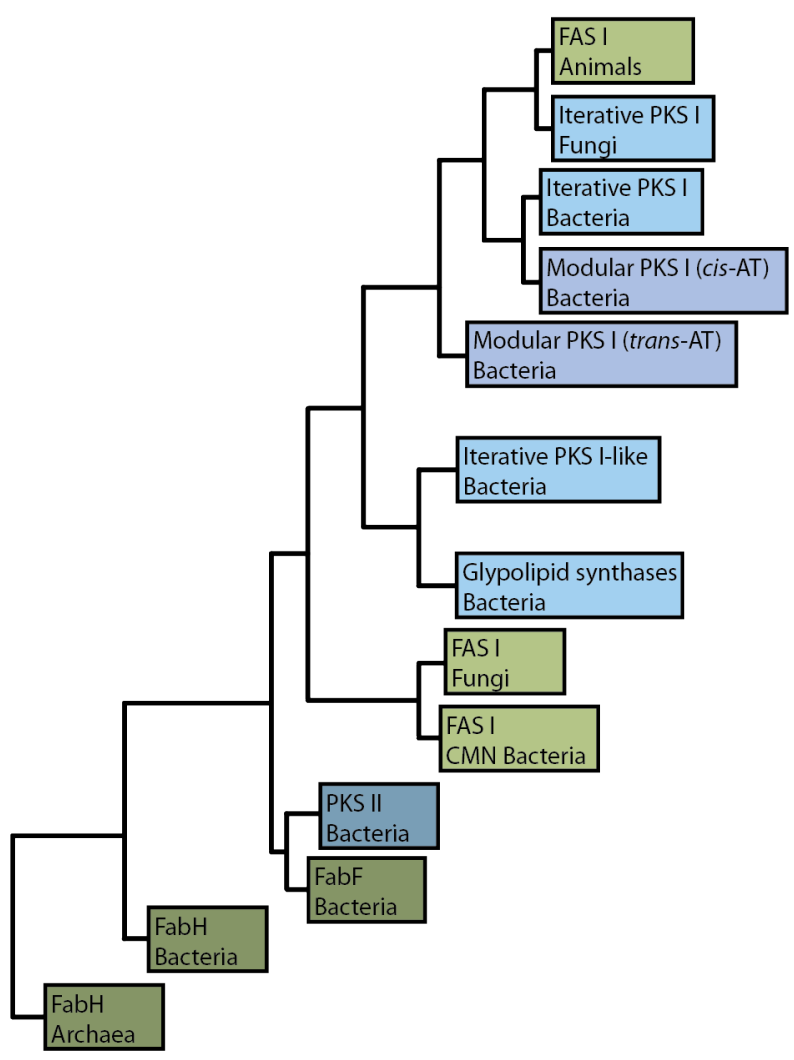

Figure 2.4: Schematic representation of the phylogenetic relationship between FASs and PKSs based on the alignment of KS domains, inferred by Bayesian statistics. Typell FAS and PKS systems have predated the multidomain and multimodular type I systems. Type I systems shown in light, type Il systems shown in dark colors. FAS systems shown in green, PKS systems shown in blue. Adapted from references. $[6,42,78]$ 


\section{Introduction}

To date, there is scientific consensus on the evolution of the multifunctional type I systems from the distinct proteins of typell systems. ${ }^{[6,34,42]}$ The dominant doctrine in this context is the gene fusion hypothesis, which states that duplication and successive fusion of primordial genes occured early in the evolution resulting in type I systems prior to the divergence of prokaryotes and eukaryotes. ${ }^{[6,34]}$ An alternative scenario is the evolution of multifunctional type I FAS systems first in eukaryotes and later via horizontal gene transfer (HGT) in prokaryotes.

However, there are two separate developments involved in the evolution of type I FASs: Fungal and CMN-bacterial typel FASs evolved together, whereas the animal type I FAS evolved in parallel to type I PKS systems. ${ }^{[41]}$ The latter systems share a common ancestor, an iterative fully methylating PKS. [41,42] The evolution of PKSs is even more complex. Although there is evidence that all PKSs are evolutionary related, the precise evolutionary relationships between different PKSs remain unclear. ${ }^{[6]}$ However, the outstanding evolution of assembly line-like systems is only found in bacterial type I PKSs. Their evolution from iterative PKSs involves certainly several genetic processes like mutation, gene fusion, and gene duplication to yield multimodular PKSs and also further recombination, gene conversion, and mutation, leading to today's diversity of assembly line-like PKSs. ${ }^{[79]}$ Furthermore, it is unclear whether cis- and trans-AT PKSs evolved together or independently. ${ }^{[6]}$ However, phylogenetic analysis of KS domains yields distinct clades of cis- and trans-AT PKSs. ${ }^{[80]}$ cis-AT domains from bacterial type I PKSs were analyzed further to determine their phylogenetic relationship. ${ }^{42]}$ They were found to form two major clades, grouped by their substrate specificity: One earlier clade includes four groups of Mal-CoA-activating ATs, the other, higher evolved clade consists of one AT-group activating Mal-CoA besides three AT-groups activating MMal-CoA and rarer substrates. Thus, it can be deduced that bacterial PKS I ATs first evolved from the Mal-CoAactivating ancestor involved in fatty acid synthesis and later functional specialization led to the evolution of the second clade with modified/expanded substrate specificity. ${ }^{[42]}$ 


\subsection{Structural Comparison of Fatty Acid and Polyketide}

\section{Synthases}

Distinct evolutionary pathways of different types of FASs and PKSs are also well reflected in structural differences. It took several decades to solve large and complex structures like type I FASs from initial low-resolution structures obtained by electron microscopy (EM) ${ }^{[81]}$ to high-resolution structures determined by $\mathrm{X}$-ray crystallography. ${ }^{[38]}$ Today, the structures of fungal, ${ }^{[82,83]}$ CMN-bacterial, ${ }^{[84]}$ and animal type I FASs ${ }^{[85]}$ confirm their different evolutionary development (Fig. 2.5). As discussed above, the first two types evolved together and share many structural features: Both show a barrel-shaped, hexameric-like ii architecture with a rigid central wheel and two domes forming the reaction chambers for biosynthesis. To form such a complex structure, about $50 \%$ and $35 \%$ of the polypeptide are used as scaffolding elements in fungal and bacterial type I FASs, respectively. ${ }^{[86]}$ Although the CMN-bacterial type is encoded on single genes and does not include a C-terminally attached phosphopantetheinyl transferase (PPT), the overall architecture is very similar iii. On the contrary, animal type I FASs, which originated from another evolutionary pathway than fungal and CMN-bacterial types, show an open architecture forming highly flexible, X-shaped homodimers. ${ }^{[41,69]}$ Due to the high conformational flexibility, the ACP and TE domains remain unresolved in the crystal structure from Maier et al. ${ }^{[85]}$ The homodimers contain a condensing and a modifying wing connected by a waist region, the condensing part consisting of KS and AT domains, and the modifying wing carrying the three domains $\mathrm{KR}, \mathrm{DH}$, and $\mathrm{ER}$ responsible for modification. In contrast to the other two type I FAS systems, all enzymatic domains are exposed to the environment. The $X$-shaped dimer forms two reaction chambers with high distances between the single domains, making large-scale conformational changes necessary for efficient biosynthesis. ${ }^{[8]}$

For PKSs, especially the modular PKSs, structural elucidation is even more challenging. Due to their large size, isolation of complete modules, let alone full-length proteins, is extremely difficult. Crystallization efforts are hampered by their inherent high flexibility. ${ }^{[87]}$ Since the first high-resolution structure of a PKS domain in 2001, ${ }^{[88]}$ it took more than a decade to gain highresolution structural information on all domains from cis-AT PKSs. [89] Structures of single domains (KR, $\left.{ }^{[90,91] ~ E R, ~}{ }^{[92]} \mathrm{DH},{ }^{[93,94]} \mathrm{TE},{ }^{[88,95]} \mathrm{ACP},{ }^{[96-98]}\right)$ or didomains (KS-AT ${ }^{[99,100]}$, KR-ER ${ }^{[101]}$ ) from modular PKSs overall share a remarkable similarity with their counterparts in the mammalian FAS. ${ }^{[85,102,103]}$ Due to the high similarity and the lack of structural infor-

\footnotetext{
iiBacterial type I FASs form homohexameric barrels, whereas fungal type I FASs form heterododecameric barrels consisting of two polypeptide chains forming an $\alpha_{6} \beta_{6}$ complex.

iii Bacterial type I FASs are relatively conserved, whereas fungal systems show further diversification resulting from a late evolutionary development: $[41,86]$ Fungal types show variations on gene level being either encoded on a single gene or on genes splitted at different positions. Nevertheless, the overall architecture and assembly pathway of these variants is very similar.
} 
mation on larger PKS units, modular PKSs were often imagined as several mammalian FAS structures lined up with its segregation into the condensing and the modifying wing (Fig. 2.6: mammalian FAS). ${ }^{[85,89,91]}$ However, some structural studies revealed certain differences, questioning the role of the mammalian FAS structure as universal model. [85,87,88,93,101,104]

A

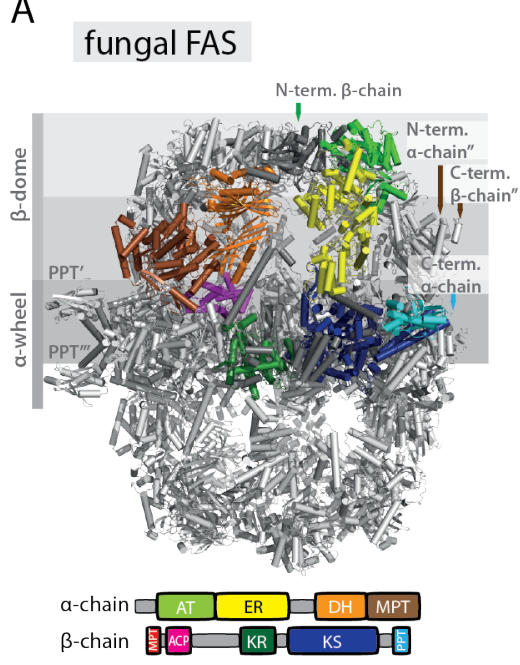

B

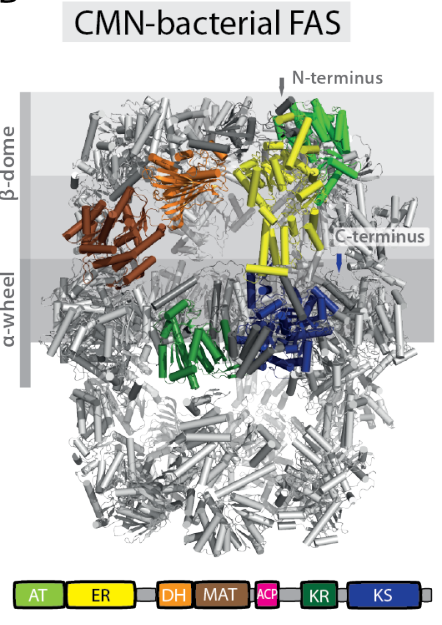

C animal FAS
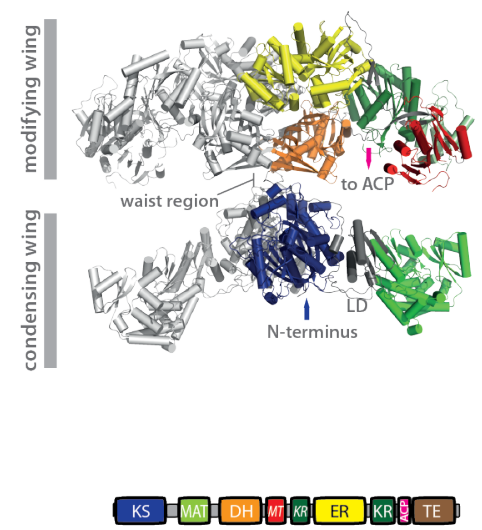

Figure 2.5: Structure and domain organization of fungal, CMN-bacterial, and mammalian type I FAS systems. (A) X-ray crystallographic model of baker's yeast FAS (PDB: 3HMJ ${ }^{[83]}$ ). (B) X-ray crystallographic model of M. smegmatis FAS (PDB: 4V8L ${ }^{[84]}$ ). (C) X-ray crystallographic model of porcine FAS (PDB: 2VZ8 ${ }^{[85]}$ ). Adapted from reference. ${ }^{[41]}$

Over time, different models for the architecture in modular PKSs emerged (Fig. 2.6). The DEBS M3 and pikromycin synthase (PikA) M5 model are based on low resolution smallangle $X$-ray scattering $(S A X S){ }^{[105]}$ and medium resolution cryo-EM data, ${ }^{[106,107]}$ respectively. These two models differ most in the relative orientation of $\mathrm{KS}$ and $\mathrm{AT}$, leading to completely different architectures. For the DEBS M3 model, the KS-AT has the same orientation as in KS-AT didomain structures solved before, $[85,99,100,103]$ but was treated as rigid body during modeling and refinement based on KS-AT data, so its configuration was invariable. Even though the shape of the rigid KS-AT body fits well with the experimental data set, it would be interesting to treat both as individual domains inspecting the actual domain orientation. ${ }^{\text {[87] In }}$ the PikA M5 model, the AT domain is positioned below the KS and rotated by approximately $120^{\circ}$ compared to other KS-AT structures, leading to an overall arch-shaped dimer with one single central reaction chamber. ${ }^{[87]}$ This module showed major discrepancies to previous models and solved structures, speaking against its role as universal model for modular PKSs. ${ }^{[108]}$ It might as well be possible that the structure represents an inactive conformation or an artifact caused by sample handling and preparation of the cryo-EM sample, which can be challenging for macromolecular complexes. [109] 


\section{Introduction}
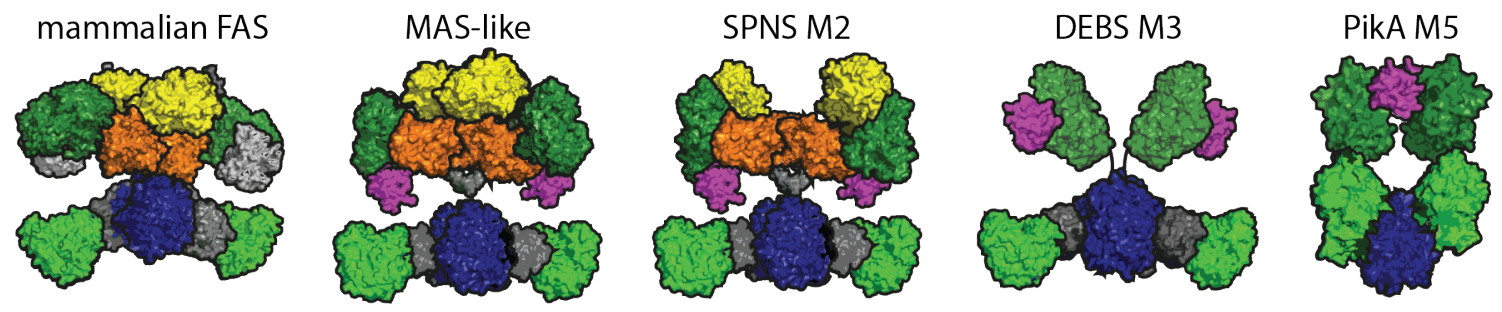

Figure 2.6: Structural models for modular PKSs. Surface representation of the X-ray structure from the mammalian FAS (PDB: 2 VZ8 ${ }^{[85]}$ ), and from a MAS-like PKS assembled from condensing and modifying wing (PDB: $5 B P 1$ and $5 B P 4{ }^{[110]}$ ), of the SPNS M2 model[101] assembled from PDBs 5BP1 (KS-AT), 5BP4 (DH), and 3SLK (ER-KR), ${ }^{[101]}$ of the SAXS data from the partially reducing module DEBS M3, ${ }^{[105]}$ and of the cryo-EM data from the partially reducing module PikAllI M5. ${ }^{[106]}$ Adapted from reference. ${ }^{[75]}$

Two other models were created by assembly of high-resolution crystal structures of the mycocerosic acid synthase (MAS)-like PKS from Mycobacterium tuberculosis ${ }^{[110]}$ and of the spinosyn synthase (SPNS) ${ }^{[101]}$ to full modules. Even though the MAS-like PKS is a fully reducing iterative PKS, it is more similar to modular PKSs (27-35\% sequence identity) than to fungal iterative PKSs (20-22\% sequence identity) and FASs (19\% sequence identity). [38] Both models are overall similar and the overall fold is related to the mammalian FAS, but they differ especially in the domains $\mathrm{DH}$ and ER. The dimeric DH domain in both modules is rather linear and resembles the dimeric DH domain of modular PKSs (interdomain angle of $\left.167-203^{\circ}\right),{ }^{[93,94,111-114]}$ whereas the DH dimer in the mammalian FAS is V-shaped. [85] Looking at the ER, this domain is dimeric in the MAS-like PKS[110] like in the mammalian FAS ${ }^{[85]}$ and some modular PKSs. ${ }^{[92,115]}$ In contrast, the ER from the SPNS and the majority of modular PKSs were found to be monomeric. ${ }^{[101,115-117]}$ The dimerization motif in ER domains and the ER-KR linker from modular PKSs are significantly shorter than those from iterative PKSs hindering ER dimerization. [115] The relevance of ER's oligomeric state in modular PKSs remains unclear, but the MAS-like PKS and the SPNS structures together may represent a relevant model for modular PKSs' scaffold.

Most recently, a high-resolution cryo-EM structure of the iterative lovastatin nonaketide synthase LovB was published (PDB: 7CPX). ${ }^{[18]}$ Like the majority of the other models, it reveals an overall $X$-shaped dimeric architecture and strongly resembles the mammalian FAS structure with V-shaped dimeric DH domains. The KS-AT forms a dimer with a rather linear conformation, which is slightly rotated compared to the mammalian FAS structure. This is most likely caused by differences in the linker domain (LD), since single domains KS and AT reveal a high structural similarity to domains in the mammalian FAS and DEBS M3. This structure supports the significance of the mammalian FAS structure in the context of type I PKS structures. 


\section{Introduction}

While all these structures give important insights into the architecture of standalone PKS modules, structural information beyond that is even rarer. Indeed, several structures of noncovalent connections between modules, the DDs, were solved $[46,47,119-121]$ and SAXS analysis gave low-resolution structures of a bimodular construct. ${ }^{[105]}$ However, high-resolution structures from full modules iv and multimodular constructs are still missing. Nevertheless, every PKS structure solved complements the picture of PKSs even if each can just be seen as one possible out of many conformations. The same also applies to protein-protein interactions which are the key to catalytic activity in megasynthases. Central domains and their interaction will be discussed in the next section.

\subsection{Key Players in Fatty Acid and Polyketide Synthesis}

$\mathrm{KS}, \mathrm{AT}$, and ACP are essential domains/enzymes and part of every FAS and PKS system or module. ${ }^{[3]}$ As mentioned above, the AT domain loads the substrates into the system onto the ACP domain. ${ }^{[9,52]}$ The ACP domain shuttles the substrate and the intermediate towards all catalytic sites. ${ }^{[57]}$ The KS domain is responsible for the condensation of the substrates to the final product. ${ }^{[58]}$ In the following, enzymatic reactions and properties of these three domains are illuminated.

\subsubsection{The Acyl Carrier Protein}

The ACP is a small, highly dynamic protein. ${ }^{[41,57]}$ It shuttles the substrates and all intermediates throughout the whole biosynthesis of fatty acids and polyketides, interacting with every catalytic enzyme/domain. [57] This protein enables the biosynthesis in the first place. Overall, ACPs from different systems share a common fold. [3]

Type I and II ACP domains from FASs were found to form helical bundles consisting of four $\alpha$-helices stabilized by inter-helical hydrophobic interactions (Fig. 2.7A). [3,122,123] Predicted and solved structures for PKSs' ACPs show a similar fold. [124-126] The inactive apo-form of the protein needs to be post-translationally modified: The conserved active site serine (S38 in rat FAS) is phosphopantetheinylated, leading to an active thiol group. ${ }^{54,55]}$ This prosthetic group with its length and high conformational flexibility is crucial for ACP's role in substrate and intermediate shuttling. [8]

\footnotetext{
iv LovB is an iterative HR-PKS, which contains a pseudo-ER, but interacts with the trans-ER LovC. The complex structure was also solved by Wang et al. (PDB: 7XPY ${ }^{[118]}$ ).
} 
A

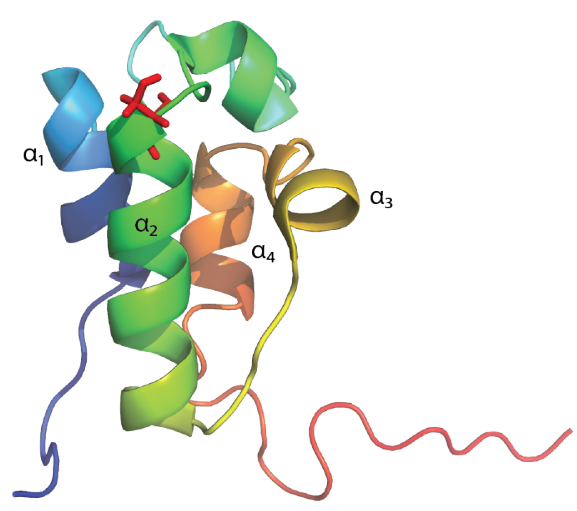

B

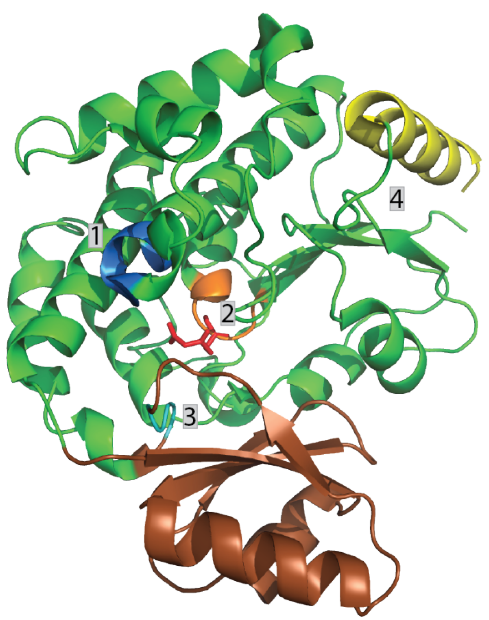

Figure 2.7: (A) Structure of the apo-ACP domain from rat FAS (PDB: 2PNG ${ }^{[102]}$ ) as cartoon representation. Coloring from blue to red corresponds to $\mathrm{N}$ - and $\mathrm{C}$-termini, respectively. ACPs consist of four helices $\alpha_{1}$ to $\alpha_{4}$. The active site S38 is highlighted in red sticks. (B) Structure of the AT domain from murine FAS (mFAS, PDB: 5MY0 ${ }^{[9]}$ ) as cartoon representation. The core domain and the subdomain are shown in green and brown, respectively. The active site S581 is highlighted in red sticks. Motifs 1 to 4 for substrate selectivity are highlighted in blue, orange, cyan, and yellow.

\subsubsection{The Acyl Transferase}

The AT is responsible for the loading of substrates into the system. [52] This domain/enzyme selects the correct substrates for fatty acid and polyketide biosynthesis out of a vast variety of compounds within the cell. In animal type I FASs, there is only one AT responsible for loading both, priming and elongation substrates. [3] Modular PKS systems, on the other hand, contain specialized ATs which discriminate between the two substrates.

More specifically, ATs catalyze the acyl transfer from CoA to the ACP. This reaction is termed transacylation and follows a ping-pong bi-bi catalytic mechanism (Fig. 2.8) catalyzed by a SerHis dyad. ${ }^{[52,127-129]}$ In the first step, the ping step, the conserved active site serine (Ser581 in murine FAS (mFAS)) attacks the carbonyl group of the substrate acyl-CoA, leading to an ATbound acyl and releasing free CoA. ${ }^{[41]}$ In the pong step, the ACP attacks the ester carbonyl, resulting in acylated ACP and the regenerated AT domain/enzyme. The nucleophilicity of the active serine is increased by a conserved histidine (His683 in mFAS) via hydrogen bonding. [41] Elongation substrates like $\mathrm{Mal}$ and $\mathrm{MMal}$ are directly interacting with a conserved arginine (Arg606 in mFAS) within the AT, facilitating its enclosure into the binding pocket. The tetrahedral intermediates of both steps are stabilized by an oxyanion hole at the active site, formed by two backbone amides (Met499 and Leu582 in mFAS). ${ }^{[130]}$ As side reaction, AT-mediated hydrolysis can occur after the ping step. [131-133] 
Using steady state assumptions, the reaction velocity is given by

$$
\mathrm{v}=\frac{\mathrm{v}_{\max }[\mathrm{X}-\mathrm{CoA}][\mathrm{ACP}]}{\mathrm{K}_{\mathrm{m}}^{\mathrm{ACP}}[\mathrm{X}-\mathrm{CoA}]+[\mathrm{X}-\mathrm{CoA}][\mathrm{ACP}]+\mathrm{K}_{\mathrm{m}}^{\mathrm{X}-\mathrm{CoA}}[\mathrm{ACP}]},
$$

with $\mathrm{v}_{\max }=\mathrm{k}_{\text {cat }}[\mathrm{AT}]_{0} \cdot[129]$

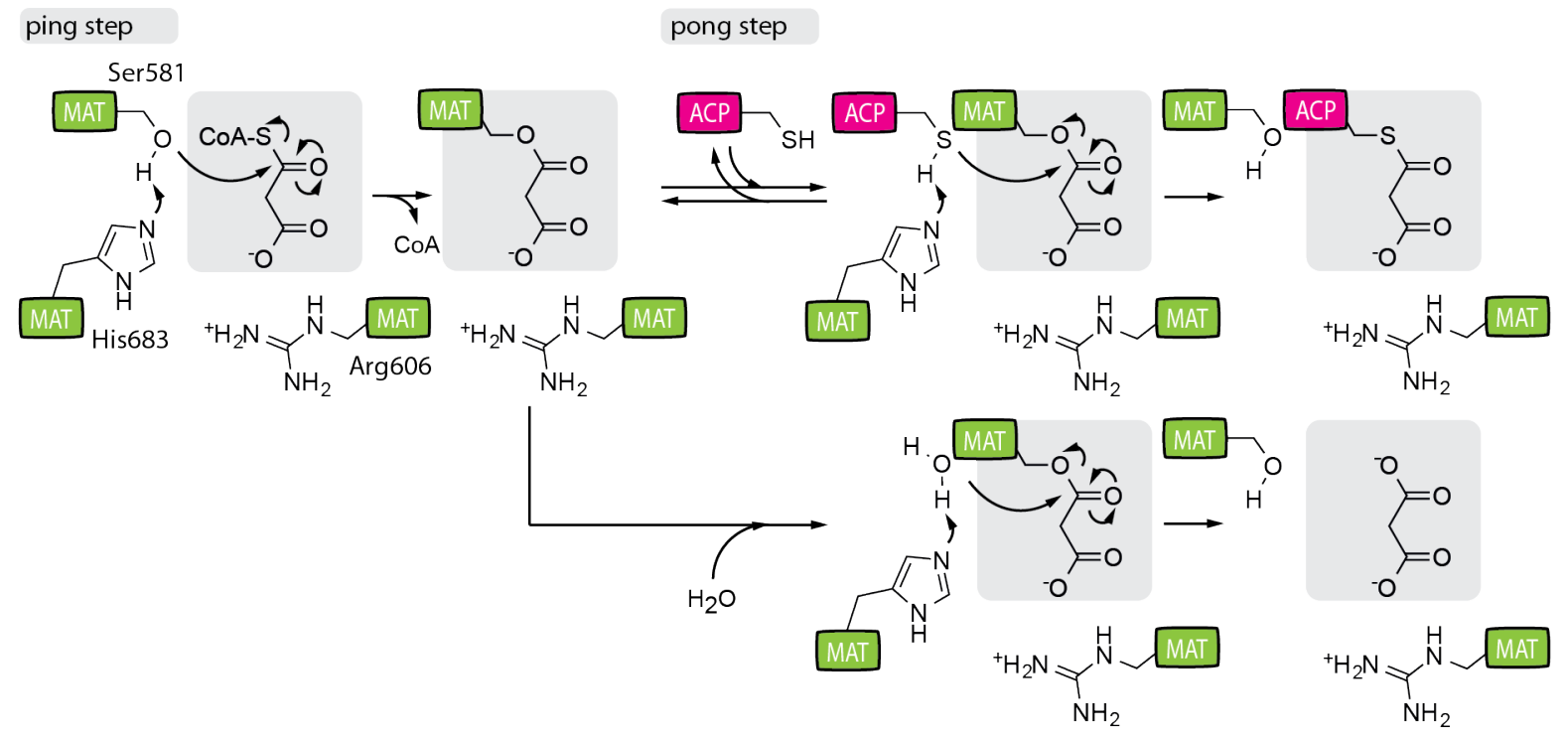

Figure 2.8: Ping-pong bi-bi reaction catalyzed by the MAT domain from mFAS: During transacylation, the acyl moiety $X$ is first loaded onto the AT, then transferred to the ACP domain. As side reaction AT-mediated hydrolysis occurs. Adapted from reference. ${ }^{[41]}$

Recently, P. Paiva and co-workers analyzed the reactions mediated by the human MAT in mechanistic detail using quantum mechanics/molecular mechanics (QM/MM) calculations. ${ }^{134,135]}$ They showed that both steps, the AT-loading ping and the ACP-loading pong step, occur in two sequential steps. The first step consists of the concerted deprotonation of Ser581 by His683 and the nuceophilic attack of the substrate thioester carbonyl carbon by the deprotonated Ser581, leading to a tetrahedral intermediate. ${ }^{[134]}$ Like proposed before, ${ }^{[130]}$ they found that this intermediate is stabilized by the interaction of the negatively charged carbonyl oxygen with the oxyanion hole formed by the backbone amides of Met499 and Leu582. The second step is the breakdown of the intermediate releasing free CoA which deprotonates the protonated His683, resulting in the acyl-loaded MAT. The third step, the first step of the pong step, consists of the concerted deprotonation of the holo-ACP thiol group by His683 and the nucleophilic attack of the MAT-bound substrate carbonyl carbon by the deprotonated thiol group. ${ }^{135]}$ The resulting tetrahedral intermediate is stabilized by the oxyanion hole. The fourth step is the breakdown of the intermediate and the deprotonation of the protonated His683 by the MAT active site Ser581 yielding acyl-loaded ACP. The computational analysis identified the first step as rate-limiting for the full MAT-mediated reaction. [135] 
Type I and II ATs from FASs and PKSs show similar folds. $[3,41,69,99,136,137]$ They comprise a core domain with an $\alpha / \beta$-hydrolase-like structure and a smaller subdomain with a ferrodoxin-like structure (Fig. 2.7B). ${ }^{[3]}$ Even though the overall structure is very similar, there are differences in the primary sequence, which affect the substrate selectivity of ATs: Animal type I FAS AT domains show a broad substrate tolerance for priming and elongation substrates. ${ }^{[9,138]}$ Likewise, priming AT domains from type I modular PKSs are strinkingly substrate tolerant. ${ }^{[3,139,140]}$ In contrast, elongation ATs exhibit a remarkable substrate selectivity and act as filter in the polyketide biosynthesis. ${ }^{[133,141-143]}$ This selectivity can be predicted from certain amino acid motifs and regions. [3]

1. The "RVDVVQ" motif: This motif is located 30 amino acids upstream from the active serine and indicates substrate specificity for MMal. Later on, it was extended to [RQSED]V[DE] VVQ, in which the first and third amino acid can vary between $R, Q, S$, $E, D$ and $D, E$, respectively. The motif for Mal specificity is ZTX\$[AT][QE], in which $Z$ is any hydrophilic residue, $\$$ is any aromatic amino acid, and the fifth and sixth amino acid vary between $A, T$ and $Q, E$, respectively. ${ }^{[3,144,145]}$

2. The "GHSXG" motif: This motif is found at the so-called "nucleophilic elbow" between a $\beta$-sheet and an $\alpha$-helix. ${ }^{[146]}$ The substrate selectivity depends on the amino acid $X$ next to the active serine. Mal-specific ATs are characterized by a branched hydrophobic amino acid like valine or isoleucine at this position. Other ATs feature a less bulky residue like glutamine or methionine due to steric restrictions of larger substrates. ${ }^{[3,144]}$ In FAS ATs which load Mal discriminating against MMal, $X$ is leucine or valine. [3]

3. The "YASH" motif: This motif is located 100 amino acids downstream from the active serine and is characteristic for MMal-specific ATs. ${ }^{[147,148]}$ Mal-specific ATs are indicated by a "HAFH" motif. These motifs were refined to [YVW]ASH and [HTVY]AFH for MMal and Mal specificity, respectively. ${ }^{[3]}$ In FAS, this motif is [MILV]AFH.

4. The C-terminal region: This region is part of an $\alpha-\beta-\alpha$ motif. The helix is part of the binding pocket entrance, but no specific sequence is defined for this motif. ${ }^{[3]} \mathrm{A}$ domain swapping study showed its role in determining the substrate specificity most likely due to its involvement in the binding pocket. ${ }^{[3,149]}$

Overall, priming ATs differ from elongation ATs mostly in the third motif. Additionally, the characteristic site arginine found in Mal- and MMal-transferring elongation ATs which interacts with the carboxylate of the elongation substrate is usually missing in priming ATs. [3] For DEBS, the priming AT shows the following amino acid sequences: 1. RVEVVQ, 2. GHSIG, and 3. MAAH. The C-terminal region is well conserved. 


\subsubsection{The $\beta$-Ketoacyl Synthase}

The KS domain catalyzes the key step in fatty acid and polyketide biosynthesis: the formation of carbon-carbon bonds. ${ }^{[150]}$ This domain enables the extension of the growing chain. Besides its major role in catalysis, the KS domain is essential for formation of the dimeric interface in FAS and PKS systems and, hence, is crucial for the architecture of these megasynthases. ${ }^{[85,110]}$ Overall, the KS domain of FASs and PKSs shows a relatively conserved primary sequence, which makes them predestined for phyologenetic analysis. ${ }^{6,42]}$

FASs' and PKSs' KS domains share a common fold, which resembles the characteristic fold of the thiolase-superfamily. [3] The KSs form homodimers; each monomer consists of two subdomains with alternating layers of $\alpha$-helices and $\beta$-sheets forming an $\alpha / \beta / \alpha / \beta / \alpha$ sandwich motif. [151] The dimer interface is stabilized by antiparallel $\beta$-strands, which exhibit hydrogen bonds. ${ }^{[3,103]} \mathrm{N}$-terminal KS domains of modular PKSs contain an additional $\alpha$-helix which forms a coiled-coil structure in the dimeric interface.

The KS catalyzes a decarboxylative Claisen condensation using a catalytic triade consisting of the catalytic cysteine and two histidines. [152] Similar as in the AT, the active site is also located at a "nucleophilic elbow" increasing its nucleophilicity. The reaction catalyzed by the KS follows a ping-pong mechanism (Fig. 2.9). [150] In the ping step, an acyl moiety is loaded into the KS' active site. This acyl moiety is either a priming substrate or a fatty acid/polyketide processed intermediate. In the pong step, the carbon-carbon bond between the KS-bound acyl and an elongation substrate is formed. For this step, there are several possible sequences of events and catalytic mechanisms: ${ }^{[41,153]}$ In the sequential decarboxylation and carbon-carbon bond formation, the elongation substrate is decarboxylated to an enolate nucleophile, which attacks the KS-bound acyl, leading to a $\beta$-ketoacyl-ACP. This sequence may be accompanied by the release of carbon dioxide or bicarbonate. ${ }^{[60]}$ Other possible scenarios are the sequential carbon-carbon bond formation and decarboxylation or the concerted reactions. To the present knowledge, the first mechanism is the predominantly preferred one. ${ }^{[41]}$ 


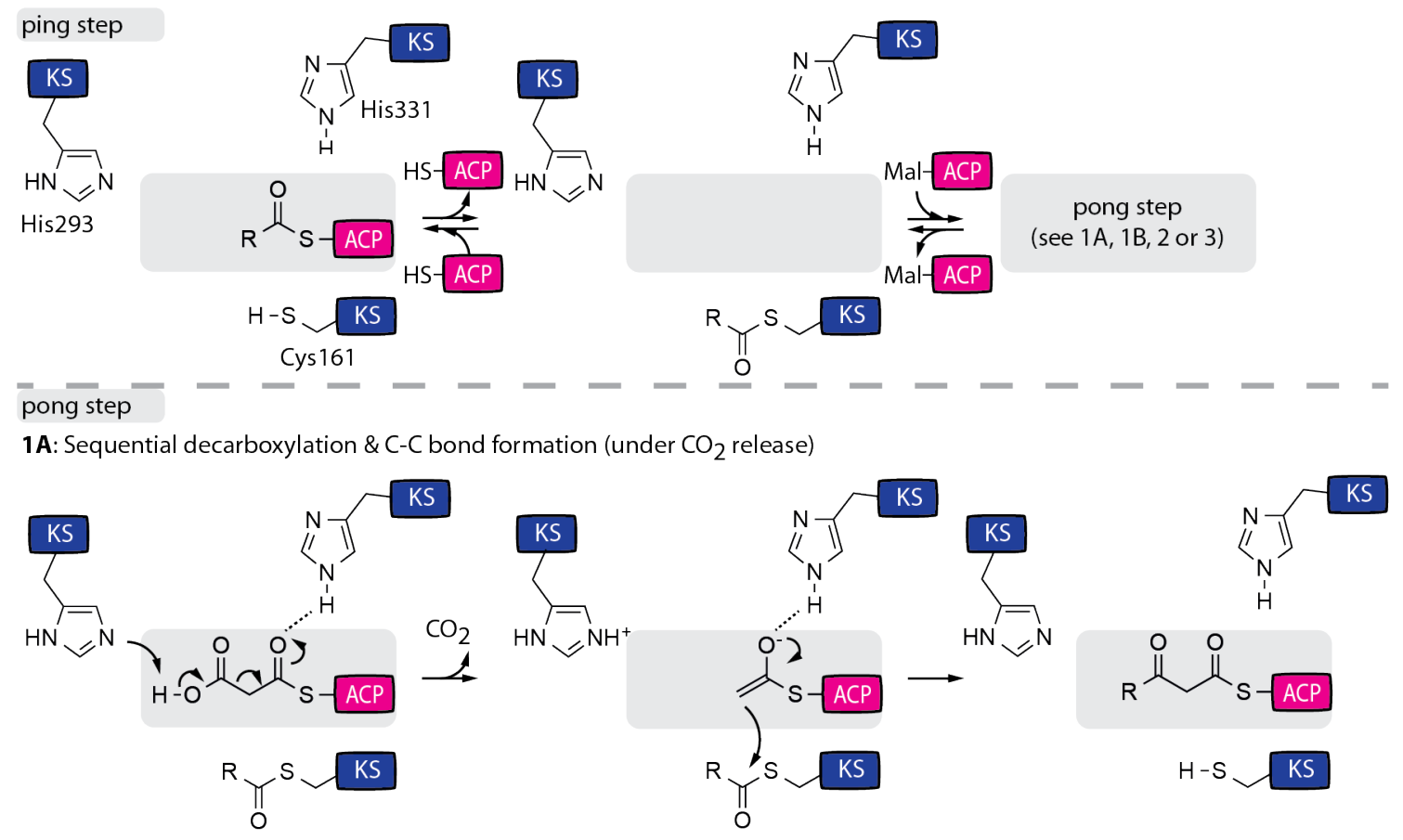

1B: Sequential decarboxylation \& C-C bond formation (under bicarbonate release)

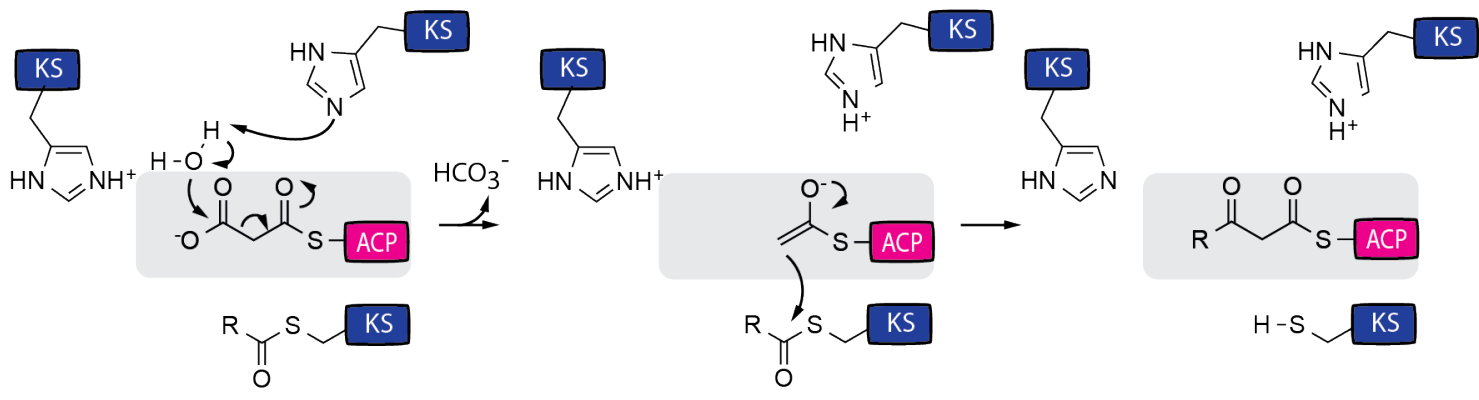

2: Sequential C-C bond formation \& decarboxylation

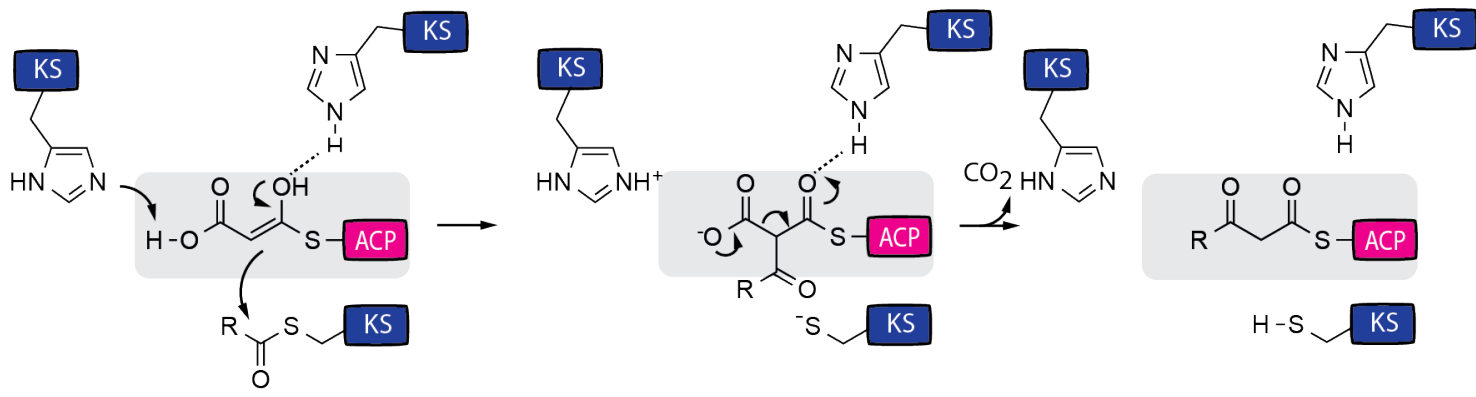

3: Concerted decarboxylation \& C-C bond formation

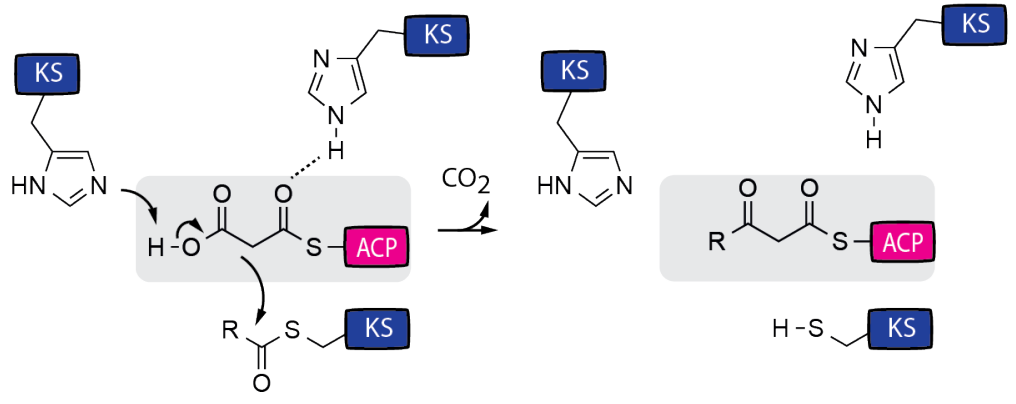


Figure 2.9: Ping-pong reaction catalyzed by the KS domain from mFAS: During transthioesterification, the acyl moiety $X$ (either priming substrate or intermediate) is first loaded onto the KS. In the pong step, the carbon-carbon bond between the acyl $X$ and an elongation substrate is formed. The detailed mechanism of the pong step is still not fully understood. Adapted from reference. ${ }^{[41]}$

$\mathrm{KSs}$ in iterative systems accept acyl moieties of different lengths from priming substrates to different processed intermediates. ${ }^{[41]}$ Since the products of fatty acid/polyketide biosynthesis show an accurately defined length, certain chain length regulation processes have to be involved. With the KS being the essential domain/enzyme responsible for carbon-carbon bond formation, it is evident to attribute to it some kind of gatekeeper function. And indeed, structures show limited space in the KS's binding pocket. ${ }^{[41]}$ Only intermediates of certain chain lengths fit in for carbon-carbon bond formation, regulating the chain length of the final product. ${ }^{154]}$ However, it remains unclear whether this control takes place in the ping or the pong step. ${ }^{[41]}$ Besides this chain length selectivity in iterative systems, it was shown that KSs of FAS systems exhibit a certain specificity for fully reduced acyl chains. ${ }^{[59]}$ However, the KS is also able to accept $\beta$-keto groups for condensation to produce triacetic acid lactone (TAL). ${ }^{[155]}$ Overall, it was shown that the KS domains from mammalian FASs accept other priming and elongation substrates besides their native substrates Ac and Mal for condensation. [138,156,157] The substrate permissiveness of KSs from PKSs is an intrinsic property of PKS systems as they do not always use the full set of processing domains/enzymes. Indeed, it was shown that the KSs from the modular PKS DEBS tolerate a variety of substrates. [158] 


\section{Introduction}

\subsubsection{Important Domain-Domain Interactions}

Besides the catalytic domains/enzymes themselves, proper domain-domain interactions are important for functional fatty acid/polyketide biosynthesis. ${ }^{[133,159]}$ Due to the transient nature of these interactions, only few of them are structurally solved. For the human FAS, an intensive computational docking study of the ACP to its partner domains has been performed, which enables mapping its trajectory and gives structural insights into catalytic mechanisms. [159] However, this section will focus solely on the most important interfaces for the present work, namely the KS:ACP and the AT:ACP.

Recently, KS:ACP interfaces of the Escherichia coli (E. coli) type II FAS were solved. [160,161] A mutation study gives insights into the mechanistic details of chain elongation. For the AT:ACP interface, there are structures of two PKS systems available. [125,126] Both structures as well as the human FAS model show different binding motifs of the ACP towards the AT domain. A recent study on the AT:ACP interface of E. coli FAS supports the hypothesis of different AT:ACP binding modes by a mutation study, showing multiple, productive conformations. [14]

Domains lined up on a polypeptide chain of type I FASs and PKSs do not interact directly in protein folding, but are covalently connected via discrete LDs. In modular PKSs, modules of the same polypeptide are also connected by covalent linkers. Non-covalent DDs connect modules of different polypeptide chains, forming coiled-coil structures. [3]

The essential KS and AT domains are connected via a defined LD, the KS-AT linker (KAL). It contains an $\alpha-\beta$ motif, which interacts with the $\alpha-\beta-\alpha$ motif of the AT domain. This rigid LD seems to be important for the KS-ACP interaction. ${ }^{[162]}$ Downstream of the AT domain, there is the post-AT linker (PAL), which interacts with the KAL and the KS domain. Both linker regions are relevant in the context of protein engineering, the next section will focus on. ${ }^{[163,164]}$ In sections 2.2 and 2.3 , the original module boundaries of modular PKSs were shown. Each module starts with the KS domain and ends with the ACP. A recent study on four cis-AT PKSs shows the evolutionary co-migration of the ACP with the downstream, not the upstream KS. ${ }^{[165]}$ A study on trans-AT PKSs supports this observation. ${ }^{[166]}$ Accordingly, a new module definition, starting with the AT, ending with the KS, has been established. [167] While these new module boundaries may reflect the evolution of PKSs, they do not consider the functional and architectural unit described by the classic module boundaries. This will be particularly interesting for different protein engineering approaches for directed synthesis of new products as discussed in the next section. 


\subsection{Generation of New Natural Products}

In the context of resistent bacteria and drugs losing their effect, the development of new pharmaceuticals is indispensible. [30] Nature, having generated the class of polyketides of diverse functions, has always set an example and inspired scientists of different disciplines. For a long time, chemists attempted to efficiently synthesize natural products in their laboratories. [168] In 1981, R. B. Woodward and co-workers successfully synthesized and isolated Erythromycin A. They established a 50-step synthesis for four parts forming the desired compound, at least 13 people were involved in the practical part, and ended up with an overall yield of $0.00642 \%$. [169-171] This example shows how time-consuming and inefficient chemical synthesis can be, especially in the context of these complex natural products.

Concurrently, researchers came up with a different idea. They had the vision of exploiting the natural systems producing these complex compounds in order to create new derivatives with enhanced bioactivity or bioavailability or completely new functions. ${ }^{[168]}$ After the initial cloning of PKS gene clusters in the 1980s, ${ }^{172]}$ different hybrid PKSs were constructed producing hybrid molecules. [173,174] After the discovery of modular PKSs in the 1990s, ${ }^{26,175]}$ these systems seemed to be predestined for this purpose due to their assembly line-like architecture. ${ }^{[176,177]}$ Following the principle of collinearity, distinct changes in modular PKSs promise to introduce predictable changes in the product. Different mix-and-match engineering approaches emerged from their modularity and are still being followed today: rational engineering approaches like reordering, ${ }^{[178]}$ deletion, $[179,180]$ inactivation, $[181,182]$ exchanging, ${ }^{[183-185]}$ or insertion ${ }^{[186]}$ of single domains or complete modules, substrate specificity engineering, $[140,187,188]$ extended to high-throughput combinatorial approaches. $[189,190]$ Different approaches succeeded in generating modified products: Specific manipulation of domains led to modified substrate selection, ${ }^{[148,191]}$ modified chain length of the product, ${ }^{[192,193]}$ and modified state of reduction of the $\beta$-position. [194] New products were generated by PKSs with swapped modules or subunits. ${ }^{[195]}$ Despite these promising results, all these strategies often suffered from drastically low activity due to various reasons. $[6,18,196]$ New substrates, tolerated by a specific domain/module (swapping, insertion, specificity engineering) of the assembly line, or new intermediates were not tolerated by other domains/modules. ${ }^{[197,198]}$ Domains or modules were not able to communicate due to artificial intra- and intermodular protein-protein interactions. ${ }^{[178,199]}$ Rational control of stereochemistry was problematic, ${ }^{[200]}$ just to name a few challenges.

Regarding these obstacles, it became obvious that classical lab techniques cannot even rudimentarily keep up with nature's capability to evolve new PKSs for gaining new functions as response to evolutionary pressure. Thus, the idea of evolution-inspired engineering arose in the late 2000s. ${ }^{[6]}$ Since changes in domain specificity in nature appear to result from 
domain swapping by recombination, one approach attempts to use these natural splice points for engineering with traditional cloning techniques. ${ }^{201-203]}$ Other approaches generate new PKSs by natural recombination. ${ }^{[204,205]}$ Both strategies rely on the identification of conserved regions, which most likely indicate natural splice points. Regions of high sequence similarity were found in the KAL and PAL, which may explain early successes in AT swapping. ${ }^{20,79]}$

In this context, I. Abe and co-workers investigated the evolutionary comigration of PKSs (see section 2.6.4). [165] This study supports the role of the PAL as natural splice point. Based on this, new module boundaries were defined and used to create hybrid assembly lines with limited success. ${ }^{[167,206]}$ Three hybrid assembly lines were tested and showed increased activity (23-50 times) using the updated module boundaries compared to the traditional boundaries, but overall product formation was significantly decreased to 40,30 , and $2.4 \%$ of wild type activity. More studies are needed in order to reveal whether these new module boundaries also reflect the functional not only the evolutionary unit.

From this short outline it becomes evident that generation of new PKSs in order to synthesize modified products in a controlled and efficient manner is still challenging. This is mainly due to limited knowledge of the highly dynamic protein-protein interactions. Extensive studies on structure and function of these complex megasynthases were not able to illuminate biochemical details and the mechanistic basis of domain interplay remains obscure.

\subsection{Motivation Behind This Thesis}

The overall goal of this research project is the modification of biosynthetic pathways in megasynthases. More precisely, the project aims for converting the mFAS into an assembly line, like modular PKSs, in order to efficiently produce designer compounds with certain functions. In this context, some questions arise.

1. If nature uses modular PKSs as assembly lines, why should we even try to use the iterative mFAS for vectorial synthesis?

2. Why should the mFAS of all FASs be used in this approach?

First of all, FASs feature higher turnover numbers in their biosynthesis than PKSs, which might be due to their involvement in primary metabolism. This is important in the context of product yields. Furthermore, FASs are not as complex as PKSs and are well characterized in structure and function. This project is based on years of experience with FAS characterization and engineering. Previous investigation revealed the special suitability of the mFAS for this research approach due to its open architecture: Domains can easily be deleted, which is required for partly reducing or non-reducing modules. ${ }^{[17]}$ 


\section{Introduction}

Converting the iterative mFAS into an assembly line is challenging and several aspects need to be addressed (Fig. 2.10):

1. The loading function of the AT domain needs to be splitted into specific loading of priming and specific loading of elongation substrates. This can be achieved either by direct mutagenesis of the AT domain or by AT domain swapping. Mutant R606A was shown to be very effective in priming the ACP, loading the priming substrate Ac three times faster than the elongation substrate Mal. ${ }^{[9,19]}$ This transferase mutant can be used in a priming module.

2. Elongation modules with varying domain compositions need to be designed. As mentioned above, the mFAS fold allows for deletion of domains. ${ }^{[17]}$

3. The interaction and communication between different modules need to be ensured. Covalent and non-covalent linkers need to be designed. First bimodular constructs with covalent linkers were already successfully designed. [17] Recent studies have shown the suitability of natural DDs from PKS DEBS and of synthetic coiled-coils, termed SYNZIPs, ${ }^{207,208]}$ for non-covalently bridging non-native interfaces in engineered PKSs. ${ }^{[163,209]}$ These linkers could be installed in the intended mFAS assembly line.

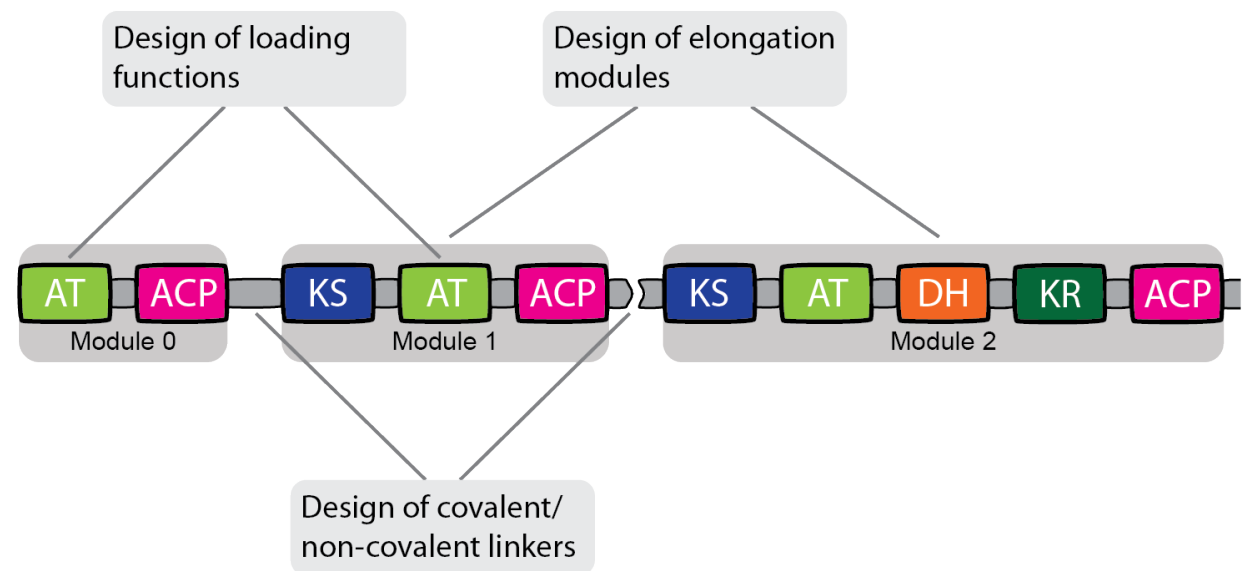

Figure 2.10: Schematic representation of the mFAS-based assembly line consisting of a priming and several exemplary elongation modules. Engineering aspects of the research plan are highlighted. 


\subsection{Aim}

The present thesis is embedded in the previously described research project with a focus mainly on installation of the loading function in the elongation module via AT domain swapping and partly the design of an elongation module. Prior to AT swapping approaches, it is essential to understand this central domain in more detail. At the beginning of my doctoral studies, little was known about kinetics in PKSs, whereas the mFAS AT domain had been characterized in depth in our group. [9]

My doctoral studies can basically be divided into two subprojects. The first is dedicated to the kinetic characterization of the AT domain from different PKSs. A fluorescence assay for kinetic analysis of ATs was already established in my master's thesis and should be further optimized during my doctoral studies. This comparative study should give deep insight into the kinetics of the complex and essential mechanism of substrate loading in PKSs. Furthermore, it should shed light on the relationship of FAS and PKS systems by revealing similarities and differences in kinetics. This first subproject is essential for FAS/PKS engineering and lays the foundation for the second subproject.

The second subproject focuses on the design of an elongation module for the artificial FAS assembly line. It mainly addresses one engineering aspect, the installation of a loading function in the mFAS elongation module via domain swapping. Based on the findings gained in the first subproject, potential candidates for domain swapping approaches should be identified. AT domains should be swapped in the mFAS, creating an elongation module. This module is of reduced complexity as it is allowed to keep its iterative working mode and does not have to interact with downstream modules, yet. Its functionality should be probed and, ideally, the interplay between priming and elongation module should be tested in first experiments. This last part is especially challenging, since it addresses all three engineering aspects mentioned above. 


\section{Results}

Parts of this chapter have been previously accepted for publication in: F. Stegemann, M. Grininger, "Transacylation Kinetics in Fatty Acid and Polyketide Synthases and its Sensitivity to Point Mutations", ChemCatChem (2021). [1] For individual contributions, copyright, and Creative Commons license, please see the statement of personal contributions.

\subsection{Preliminary Work}

As described above, the ATs as central domains in all FAS and PKS systems are attractive targets for protein engineering. They are gatekeepers for loading these protein complexes and control product synthesis as selectivity filters. Key for successful engineering strategies is detailed mechanistic understanding. During my master's thesis, ${ }^{[210]}$ we established an enzymecoupled assay to quantify transferase activity, based on previous work. ${ }^{133,211]}$ In the course of my doctoral studies, we further optimized this assay and subsequently used it to compare the AT domains of different FAS and PKS systems.

\subsection{Kinetic Description of AT-mediated Reactions}

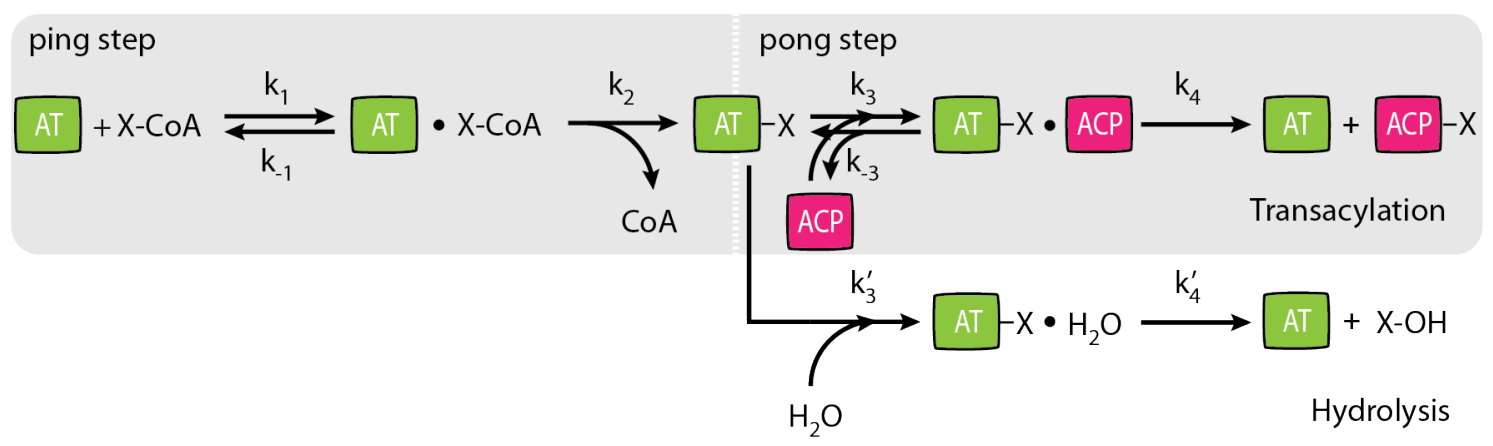

Figure 3.1: Schematic representation of AT-mediated reactions. After formation of the first enzyme-substrate complex AT.X-CoA, free CoA is released and AT-X is formed. The substrate $X$ is either transacylated via formation of the second enzyme-substrate complex AT-X.ACP (main branch) or hydrolyzed during AT-mediated hydrolysis (side branch). The kinetic constants $\mathrm{k}_{1}$ to $\mathrm{k}_{4}$ as well as $\mathrm{k}_{-1}, \mathrm{k}_{-3}, \mathrm{k}_{3}{ }_{3}$, and $\mathrm{k}_{4}{ }_{4}$ describe the AT-mediated reactions. 


\section{Results}

The AT domain catalyzes the transfer of the acyl moiety $X$ of the substrate $X-C o A$ onto the holo-ACP domain (Fig. 3.1). This transacylation reaction follows a double displacement mechanism, called ping-pong bi-bi mechanism. ${ }^{[129]}$ Two substrates (X-CoA and ACP) are involved leading to two products ( $C O A$ and $X-A C P)$. The double displacement mechanism starts with the ping step, in which the acyl moiety $X$ is first transferred to the $A T$, resulting in release of product CoA. The pong step denotes the subsequent transfer of the acyl moiety $X$ to the ACP after binding the second substrate ACP to AT- $X$, leading to the final product ACP-X. Hydrolysis occurs as side reaction, releasing the free acid " $\mathrm{X}-\mathrm{OH}$ ". The mathematical description of these reactions is given in the following.

\subsubsection{Transacylation}

AT mediates the loading of the acyl moiety $\mathrm{X}$ onto the ACP domain via a double discplacement reaction:

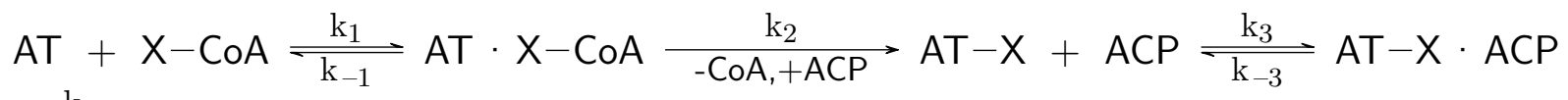
$\stackrel{\mathrm{k}_{4}}{\longrightarrow} \mathrm{AT}+\mathrm{X}-\mathrm{ACP}$

Notation:

$$
\begin{array}{llll}
\mathrm{c}_{1}:=[\mathrm{AT}], & \tilde{\mathrm{c}}_{1}:=[\mathrm{X}-\mathrm{CoA}], & \mathrm{c}_{2}:=[\mathrm{AT} \cdot \mathrm{X}-\mathrm{CoA}], & \mathrm{c}_{3}:=[\mathrm{AT}-\mathrm{X}], \\
\tilde{\mathrm{c}}_{3}:=[\mathrm{ACP}], & \mathrm{c}_{4}:=[\mathrm{AT}-\mathrm{X} \cdot \mathrm{ACP}], & \mathrm{c}_{1,0}:=[\mathrm{AT}]_{0}, & \dot{\mathrm{c}}_{\mathrm{i}}:=\frac{\mathrm{dc}_{\mathrm{i}}}{\mathrm{dt}} .
\end{array}
$$

Please note that $c_{i}$ is a shorthand notation for $c_{i}(t)$ where $t$ denotes time. $c_{1,0}$ is a shorthand notation for $c_{1}(0)$.

The following kinetic equations describe the chemical reactions following steady state assumptions:

$$
\begin{aligned}
& \mathrm{v}:=\frac{\mathrm{d}[\mathrm{X}-\mathrm{ACP}]}{\mathrm{dt}}=\mathrm{k}_{4} \mathrm{c}_{4}, \\
& 0=\dot{\mathrm{c}}_{4}=\mathrm{k}_{3} \mathrm{c}_{3} \tilde{\mathrm{c}}_{3}-\mathrm{k}_{-3} \mathrm{c}_{4}-\mathrm{k}_{4} \mathrm{c}_{4} \Rightarrow \mathrm{k}_{3} \mathrm{c}_{3} \tilde{\mathrm{c}}_{3}=\left(\mathrm{k}_{-3}+\mathrm{k}_{4}\right) \mathrm{c}_{4}, \\
& 0=\dot{\mathrm{c}}_{3}=\mathrm{k}_{2} \mathrm{c}_{2}-\mathrm{k}_{3} \mathrm{c}_{3} \tilde{\mathrm{c}}_{3}+\mathrm{k}_{-3} \mathrm{c}_{4} \quad \Rightarrow \mathrm{k}_{3} \mathrm{c}_{3} \tilde{\mathrm{c}}_{3}=\mathrm{k}_{2} \mathrm{c}_{2}+\mathrm{k}_{-3} \mathrm{c}_{4}, \\
& 0=\dot{\mathrm{c}}_{2}=\mathrm{k}_{1} \mathrm{c}_{1} \tilde{\mathrm{c}}_{1}-\mathrm{k}_{-1} \mathrm{c}_{2}-\mathrm{k}_{2} \mathrm{c}_{2}
\end{aligned}
$$


Mathematical transformations:

$(3.2)=(3.3) \quad\left(\mathrm{k}_{-3}+\mathrm{k}_{4}\right) \mathrm{c}_{4}=\mathrm{k}_{2} \mathrm{c}_{2}+\mathrm{k}_{3} \mathrm{c}_{4} \quad \Rightarrow \mathrm{c}_{2}=\frac{\mathrm{k}_{4}}{\mathrm{k}_{2}} \mathrm{c}_{4}$,

$\mathrm{c}_{2}$ into (3.4) $\quad \mathrm{k}_{1} \mathrm{c}_{1} \tilde{\mathrm{c}}_{1}=\left(\mathrm{k}_{-1}+\mathrm{k}_{2}\right) \frac{\mathrm{k}_{4}}{\mathrm{k}_{2}} \mathrm{c}_{4}$,

$\mathrm{c}_{2}$ into (3.5) $\quad \mathrm{c}_{1}=\mathrm{c}_{1,0}-\mathrm{c}_{3}-\frac{\mathrm{k}_{2}+\mathrm{k}_{4}}{\mathrm{k}_{2}} \mathrm{c}_{4}$,

$\mathrm{c}_{1} \operatorname{into}(3.7) \quad \mathrm{k}_{1} \tilde{\mathrm{c}}_{1}\left(\mathrm{c}_{1,0}-\mathrm{c}_{3}-\frac{\mathrm{k}_{2}+\mathrm{k}_{4}}{\mathrm{k}_{2}} \mathrm{c}_{4}\right)=\left(\mathrm{k}_{-1}+\mathrm{k}_{2}\right) \frac{\mathrm{k}_{4}}{\mathrm{k}_{2}} \mathrm{c}_{4}$

$$
\Rightarrow \tilde{\mathrm{c}}_{1} \mathrm{c}_{3}=\mathrm{c}_{1,0} \tilde{\mathrm{c}}_{1}-\left(\frac{\mathrm{k}_{2}+\mathrm{k}_{4}}{\mathrm{k}_{2}} \tilde{\mathrm{c}}_{1}+\frac{\mathrm{k}_{4}\left(\mathrm{k}_{-1}+\mathrm{k}_{2}\right)}{\mathrm{k}_{1} \mathrm{k}_{2}}\right) \mathrm{c}_{4},
$$

$(3.2) \times \tilde{\mathrm{c}}_{1} \quad \mathrm{k}_{3} \tilde{\mathrm{c}}_{1} \mathrm{c}_{3} \tilde{\mathrm{c}}_{3}=\left(\mathrm{k}_{-3}+\mathrm{k}_{4}\right) \tilde{\mathrm{c}}_{1} \mathrm{c}_{4}$,

(3.9) into (3.10) $\quad \mathrm{k}_{3} \tilde{\mathrm{c}}_{3}\left[\mathrm{c}_{1,0} \tilde{\mathrm{c}}_{1}-\left(\frac{\mathrm{k}_{2}+\mathrm{k}_{4}}{\mathrm{k}_{2}} \tilde{\mathrm{c}}_{1}+\frac{\mathrm{k}_{4}\left(\mathrm{k}_{-1}+\mathrm{k}_{2}\right)}{\mathrm{k}_{1} \mathrm{k}_{2}}\right) \mathrm{c}_{4}\right]=\left(\mathrm{k}_{-3}+\mathrm{k}_{4}\right) \tilde{\mathrm{c}}_{1} \mathrm{c}_{4}$

$$
\begin{gathered}
\Rightarrow \mathrm{k}_{3} \mathrm{c}_{1,0} \tilde{\mathrm{c}}_{3} \tilde{\mathrm{c}}_{1}=\mathrm{c}_{4} \\
\times\left(\frac{\mathrm{k}_{3} \mathrm{k}_{4}\left(\mathrm{k}_{-1}+\mathrm{k}_{2}\right)}{\mathrm{k}_{1} \mathrm{k}_{2}} \tilde{\mathrm{c}}_{3}+\frac{\mathrm{k}_{3}\left(\mathrm{k}_{2}+\mathrm{k}_{4}\right)}{\mathrm{k}_{2}} \tilde{\mathrm{c}}_{3} \tilde{\mathrm{c}}_{1}+\left(\mathrm{k}_{-3}+\mathrm{k}_{4}\right) \tilde{\mathrm{c}}_{1}\right) \\
\quad=\frac{\mathrm{k}_{3}\left(\mathrm{k}_{2}+\mathrm{k}_{4}\right)}{\mathrm{k}_{2}} \mathrm{c}_{4} \\
\quad \times\left(\frac{\mathrm{k}_{4}\left(\mathrm{k}_{-1}+\mathrm{k}_{2}\right)}{\mathrm{k}_{1}\left(\mathrm{k}_{2}+\mathrm{k}_{4}\right)} \tilde{\mathrm{c}}_{3}+\tilde{\mathrm{c}}_{3} \tilde{\mathrm{c}}_{1}+\frac{\mathrm{k}_{2}\left(\mathrm{k}_{-3}+\mathrm{k}_{4}\right)}{\mathrm{k}_{3}\left(\mathrm{k}_{2}+\mathrm{k}_{4}\right)} \tilde{\mathrm{c}}_{1}\right) \\
\Rightarrow \mathrm{c}_{4}=\frac{\mathrm{k}_{2}}{\frac{\mathrm{k}_{4}\left(\mathrm{k}_{-1}+\mathrm{k}_{2}\right)}{\left.\mathrm{k}_{1}+\mathrm{k}_{2}+\mathrm{k}_{4}\right)} \tilde{\mathrm{c}}_{3}+\tilde{\mathrm{c}}_{3} \tilde{\mathrm{c}}_{3} \tilde{\mathrm{c}}_{1}+\frac{\mathrm{k}_{2}\left(\mathrm{k}_{-3}+\mathrm{k}_{4}\right)}{\mathrm{k}_{3}\left(\mathrm{k}_{2}+\mathrm{k}_{4}\right)} \tilde{\mathrm{c}}_{1}}, \\
\mathrm{c}_{4} \text { into (3.1) } \quad \mathrm{v}=\frac{\frac{\mathrm{k}_{2} \mathrm{k}_{4}}{\mathrm{k}_{2}+\mathrm{k}_{4}} \mathrm{c}_{1,0} \tilde{\mathrm{c}}_{3} \tilde{\mathrm{c}}_{1}}{\frac{\mathrm{k}_{4}\left(\mathrm{k}_{-1}+\mathrm{k}_{2}\right)}{\mathrm{k}_{1}\left(\mathrm{k}_{2}+\mathrm{k}_{4}\right)} \tilde{\mathrm{c}}_{3}+\tilde{\mathrm{c}}_{3} \tilde{\mathrm{c}}_{1}+\frac{\mathrm{k}_{2}\left(\mathrm{k}_{-3}+\mathrm{k}_{4}\right)}{\mathrm{k}_{3}\left(\mathrm{k}_{2}+\mathrm{k}_{4}\right)} \tilde{\mathrm{c}}_{1}} .
\end{gathered}
$$

For a double displacement reaction, the velocity equation is defined as follows:

$$
\mathrm{v}=\frac{\mathrm{v}_{\max } \tilde{\mathrm{c}}_{3} \tilde{\mathrm{c}}_{1}}{\mathrm{~K}_{\mathrm{m}}^{\tilde{\mathrm{c}}_{1} \tilde{\mathrm{c}}_{3}}+\tilde{\mathrm{c}}_{3} \tilde{\mathrm{c}}_{1}+\mathrm{K}_{\mathrm{m}}^{\tilde{\mathrm{c}}_{3}} \tilde{\mathrm{c}}_{1}} \text { with } \mathrm{v}_{\max }=\mathrm{k}_{\mathrm{cat}} \mathrm{c}_{1,0} \cdot{ }^{[129]}
$$




\section{Results}

This yields the following kinetic parameters describing the transacylation:

$$
\begin{aligned}
\mathrm{k}_{\mathrm{cat}} & =\frac{\mathrm{k}_{2} \mathrm{k}_{4}}{\mathrm{k}_{2}+\mathrm{k}_{4}}, \\
\mathrm{~K}_{\mathrm{m}}^{\mathrm{X}-\mathrm{CoA}} & =\frac{\mathrm{k}_{4}\left(\mathrm{k}_{-1}+\mathrm{k}_{2}\right)}{\mathrm{k}_{1}\left(\mathrm{k}_{2}+\mathrm{k}_{4}\right)}=\frac{\mathrm{k}_{\mathrm{cat}}}{\mathrm{k}_{1}}+\frac{\mathrm{k}_{-1} \mathrm{k}_{4}}{\mathrm{k}_{1}\left(\mathrm{k}_{2}+\mathrm{k}_{4}\right)}, \\
\frac{\mathrm{K}_{\mathrm{m}}^{\mathrm{ACP}}}{\mathrm{k}_{\mathrm{cat}}} & =\frac{\mathrm{k}_{2}\left(\mathrm{k}_{-3}+\mathrm{k}_{4}\right)}{\mathrm{k}_{3}\left(\mathrm{k}_{2}+\mathrm{k}_{4}\right)}=\frac{\mathrm{k}_{\mathrm{cat}}}{\mathrm{k}_{3}-\mathrm{CoA}}+\frac{\mathrm{k}_{2} \mathrm{k}_{-3}}{\mathrm{k}_{3}\left(\mathrm{k}_{2}+\mathrm{k}_{4}\right)}, \\
\frac{\mathrm{k}_{-1}+\mathrm{k}_{2}}{\mathrm{k}_{\mathrm{cat}}} & =\frac{\mathrm{k}_{3} \mathrm{k}_{4}}{\mathrm{k}_{-3}+\mathrm{k}_{4}} .
\end{aligned}
$$

\subsubsection{AT-mediated Hydrolysis}

As side reaction to transacylation, AT-mediated hydrolysis occurs. Both reaction pathways include the acyl transfer, during which free $\mathrm{CoA}$ is released. After loading the acyl moiety $X$ onto the AT domain, the former is either transferred to the ACP or hydrolyzed. This AT-mediated hydrolysis follows these chemical reactions:

$$
\begin{aligned}
& \mathrm{AT}+\mathrm{X}-\mathrm{CoA} \underset{\mathrm{k}_{-1}}{\stackrel{\mathrm{k}_{1}}{\mathrm{k}_{4}}} \mathrm{AT} \cdot \mathrm{X}-\mathrm{CoA} \frac{\mathrm{k}_{2}}{-\mathrm{CoA},+\mathrm{H}_{2} \mathrm{O}} \mathrm{AT}-\mathrm{X}+\mathrm{H}_{2} \mathrm{O} \stackrel{\mathrm{k}^{\prime}{ }_{3}}{\longrightarrow} \mathrm{AT}-\mathrm{X}-\mathrm{OH} \cdot \mathrm{H}_{2} \mathrm{O}
\end{aligned}
$$

Notation:

$$
\begin{aligned}
& \mathrm{c}_{1}:=[\mathrm{AT}], \quad \tilde{\mathrm{c}}_{1}:=[\mathrm{X}-\mathrm{CoA}], \quad \mathrm{c}_{2}:=[\mathrm{AT} \cdot \mathrm{X}-\mathrm{CoA}], \quad \mathrm{c}_{3}:=[\mathrm{AT}-\mathrm{X}], \\
& \tilde{\mathrm{c}}_{3}^{\prime}:=\left[\mathrm{H}_{2} \mathrm{O}\right], \quad \mathrm{c}_{4}^{\prime}:=\left[\mathrm{AT}-\mathrm{X} \cdot \mathrm{H}_{2} \mathrm{O}\right], \quad \mathrm{c}_{1,0}:=[\mathrm{AT}]_{0}, \quad \dot{\mathrm{c}}_{\mathrm{i}}:=\frac{\mathrm{dc}_{\mathrm{i}}}{\mathrm{dt}} .
\end{aligned}
$$

The derivation is done analogously, the following parameters change from transacylation to hydrolysis:

$\tilde{\mathrm{c}}_{3} \rightarrow \tilde{\mathrm{c}}_{3}^{\prime}, \quad \mathrm{c}_{4} \rightarrow \mathrm{c}_{4}^{\prime}, \quad \mathrm{k}_{3} \rightarrow \mathrm{k}_{3}^{\prime}, \quad \mathrm{k}_{4} \rightarrow \mathrm{k}_{4}^{\prime}, \quad \mathrm{k}_{-3} \rightarrow 0$. 
This gives the following reaction velocity:

$$
\mathrm{v}=\frac{\frac{\mathrm{k}_{2} \mathrm{k}_{4}^{\prime}}{\mathrm{k}_{2}+\mathrm{k}_{4}^{\prime}} \mathrm{c}_{1,0} \tilde{\mathrm{c}}_{3}^{\prime} \tilde{\mathrm{c}}_{1}}{\frac{\mathrm{k}_{4}^{\prime}\left(\mathrm{k}_{-1}+\mathrm{k}_{2}\right)}{\mathrm{k}_{1}\left(\mathrm{k}_{2}+\mathrm{k}_{4}^{\prime}\right)} \tilde{\mathrm{c}}_{3}^{\prime}+\tilde{\mathrm{c}}_{3}^{\prime} \tilde{\mathrm{c}}_{1}+\frac{\mathrm{k}_{2} \mathrm{k}_{4}^{\prime}}{\mathrm{k}_{3}^{\prime}\left(\mathrm{k}_{2}+\mathrm{k}_{4}^{\prime}\right)} \tilde{\mathrm{c}}_{1}}
$$

Setting $\tilde{c}_{3}^{\prime} \approx 1$, since working in aqueous solution, gives $\mathrm{v}$ as follows:

$$
\mathrm{v}=\frac{\frac{\mathrm{k}_{2} \mathrm{k}_{4}^{\prime}}{\mathrm{k}_{2}+\mathrm{k}_{4}^{\prime}} \mathrm{c}_{1,0} \tilde{\mathrm{c}}_{1}}{\frac{\mathrm{k}_{4}^{\prime}\left(\mathrm{k}_{-1}+\mathrm{k}_{2}\right)}{\mathrm{k}_{1}\left(\mathrm{k}_{2}+\mathrm{k}_{4}^{\prime}\right)}+\left[1+\frac{\mathrm{k}_{2} \mathrm{k}_{4}^{\prime}}{\mathrm{k}_{3}^{\prime}\left(\mathrm{k}_{2}+\mathrm{k}_{4}^{\prime}\right)}\right] \tilde{\mathrm{c}}_{1}}=\frac{\frac{\mathrm{k}_{2} \mathrm{k}_{3}^{\prime} \mathrm{k}_{4}^{\prime}}{\mathrm{k}_{2} \mathrm{k}_{4}^{\prime}+\mathrm{k}_{3}^{\prime}\left(\mathrm{k}_{2}+\mathrm{k}_{4}^{\prime}\right)} \mathrm{c}_{1,0} \tilde{\mathrm{c}}_{1}}{\frac{\mathrm{k}_{3}^{\prime} \mathrm{k}_{4}^{\prime}\left(\mathrm{k}_{-1}+\mathrm{k}_{2}\right)}{\mathrm{k}_{1}\left[\mathrm{k}_{2} \mathrm{k}_{4}^{\prime}+\mathrm{k}_{3}^{\prime}\left(\mathrm{k}_{2}+\mathrm{k}_{4}^{\prime}\right)\right]}+\tilde{\mathrm{c}}_{1}} .
$$

This yields the following kinetic parameters describing hydrolysis. Notably, $\frac{k_{c a t}}{\mathrm{~K}_{\mathrm{m}}^{\mathrm{X}-\mathrm{CoA}}}$ is the same as for transacylation:

$$
\begin{aligned}
\mathrm{k}_{\mathrm{cat}} & =\frac{\mathrm{k}_{2} \mathrm{k}_{3}^{\prime} \mathrm{k}_{4}^{\prime}}{\mathrm{k}_{2} \mathrm{k}_{4}^{\prime}+\mathrm{k}_{3}^{\prime}\left(\mathrm{k}_{2}+\mathrm{k}_{4}^{\prime}\right)}, \\
\mathrm{K}_{\mathrm{m}}^{\mathrm{X}-\mathrm{CoA}} & =\frac{\mathrm{k}_{3}^{\prime} \mathrm{k}_{4}^{\prime}\left(\mathrm{k}_{-1}+\mathrm{k}_{2}\right)}{\mathrm{k}_{1}\left[\mathrm{k}_{2} \mathrm{k}_{4}^{\prime}+\mathrm{k}_{3}^{\prime}\left(\mathrm{k}_{2}+\mathrm{k}_{4}^{\prime}\right)\right]}=\frac{\mathrm{k}_{\mathrm{cat}}}{\mathrm{k}_{1}}+\frac{\mathrm{k}_{-1} \mathrm{k}_{3}^{\prime} \mathrm{k}_{4}^{\prime}}{\mathrm{k}_{1}\left[\mathrm{k}_{2} \mathrm{k}_{4}^{\prime}+\mathrm{k}_{3}^{\prime}\left(\mathrm{k}_{2}+\mathrm{k}_{4}^{\prime}\right)\right]}, \\
\frac{\mathrm{k}_{\mathrm{cat}}}{\mathrm{K}_{\mathrm{m}}^{\mathrm{X}-\mathrm{CoA}}} & =\frac{\mathrm{k}_{1} \mathrm{k}_{2}}{\mathrm{k}_{-1}+\mathrm{k}_{2}} .
\end{aligned}
$$




\subsection{Kinetic Analysis of Acyl Transferases}

As part of my master's thesis, we established a fluorescence assay, which was further optimized during my doctoral studies. The transacylation involves two substrates and can be seen as two sequential Michaelis-Menten reactions, which can be kinetically analyzed by titration of substrates. Titration of the acyl substrate to a series of fixed ACP concentrations (ACP titration) allows for quantification of transacylation activity. Via the velocity equation (3.13) describing this reaction, the data can be plotted globally, ${ }^{212]}$ determining the kinetic parameters described in equations (3.15) to (3.19). For quantification of AT-mediated hydrolysis, X-CoA is titrated in the absence of ACP. The corresponding Michaelis-Menten plot gives access to the kinetic parameters described in equations (3.22) to (3.24).

\subsubsection{Selection of Acyl Transferases}

To understand similarities and differences between types of FASs and PKSs, the following AT domains were selected for analysis: Representing elongation ATs of modular PKSs, the AT of DEBS M5 from Saccharopolyspora erythraea (S. erythraea) (termed DEBS3M5), of PikA M5 from Streptomyces venezuelae (PikAllIM5), and of rapamycin synthase (RAPS) module 14 from Streptomyces hygroscopicus (RAPS3M14) were used. The first two ATs transfer MMal, while the latter transfers Mal. ${ }^{[4,87]}$ Priming ATs of DEBS and avermectin PKS (DEBS1M0 and AVES1M0) were used, loading Prop and 2-methylbutyryl (2-MB). For the iterative PKSs, we worked with the AT of Pks7 from S. erythraea. Pks7 AT is phylogenetically similar to 6methylsalicylic acid synthase (MSAS) AT from Penicillium patulum ${ }^{[213]}$ and is likely involved in methylsalicylic acid (MSA) synthesis (Fig. 3.2). ${ }^{[214]}$ Thus, it is expected to load Ac and Mal onto the ACP for MSA production. ${ }^{[3,215]}$ The specificity for Mal, and not MMal, is supported by its amino acid fingerprint (Fig. 3.3). [3] Data will be compared to the Mal-loading type II FAS AT from E. coli (AT termed FabD) and to the previously analyzed type I mFAS AT (mMAT), which was found to be promiscuous in transferring a large variety of substrates. [9] If not explicitly mentioned otherwise, the terms FAS and PKS will refer to type I FAS and type I PKS in the following. 


\section{Results}

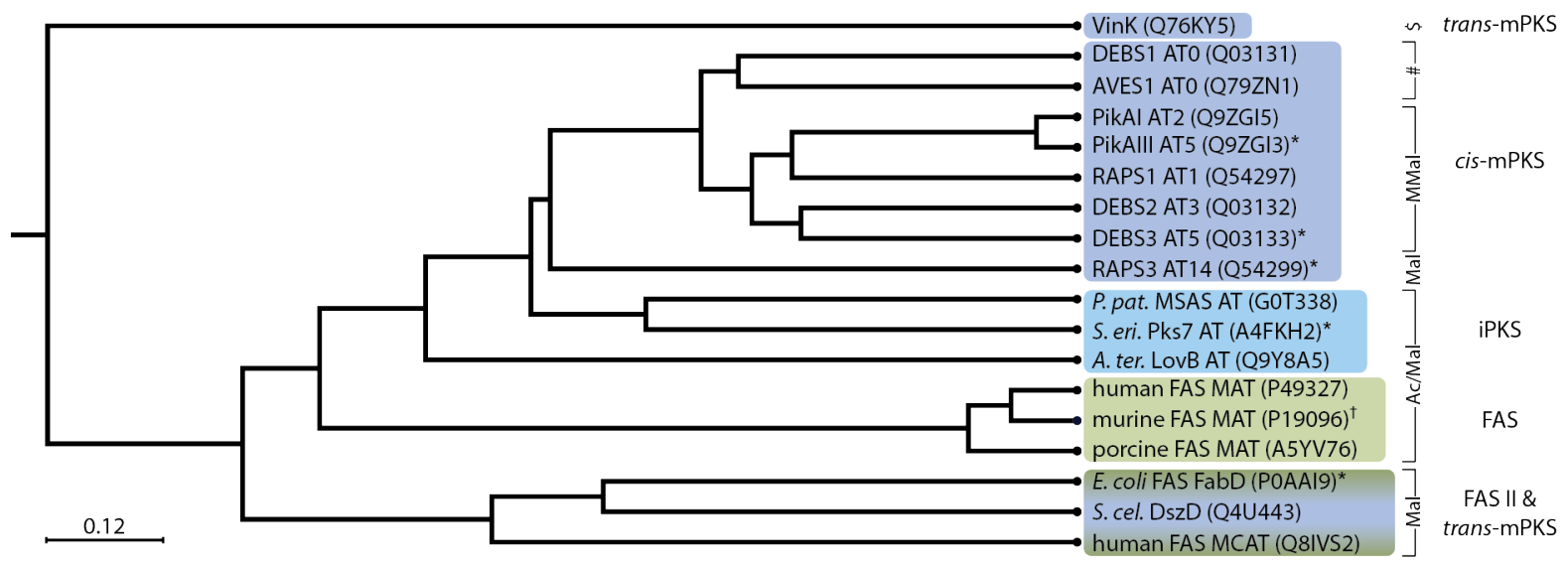

Figure 3.2: Schematic representation of the phylogenetic relationship between AT domains from FASs and PKSs (UniProt IDs in brackets). ATs from modular PKSs form a distinct clade with a subclade for the priming ATs (\# indicates priming substrates: Prop for DEBS1 AT0 and 2-MB for AVES1 AT0). As expected, RAPS3 AT14 accepting Mal is separated from the MMal-transferring ATs. ATs from iterative PKSs fall into a distinct clade. FAS II ATs form a distinct clade with the trans-acting AT DszD from disorazole PKS. All three ATs transfer Mal as substrate. The trans-acting AT VinK from vicenistatin PKS transfers an unusual substrate - a dipeptidyl moiety (indicated by $\$)^{[125]}$ - and falls into a different clade. FAS systems depicted in green (typel light green, type II dark green), iterative and modular PKS systems (iPKS and mPKS) shown in blue and violet, respectively. ATs analyzed in this study are indicated by *. The MAT analyzed in a previous study and discussed in this study is marked with ${ }^{\dagger}$.9] Phylogenetic tree created using CLC Main Workbench 6.9.1 (tree construction method: UPGMA, protein distance measure: Jukes-Cantor, bootstrap value=100).

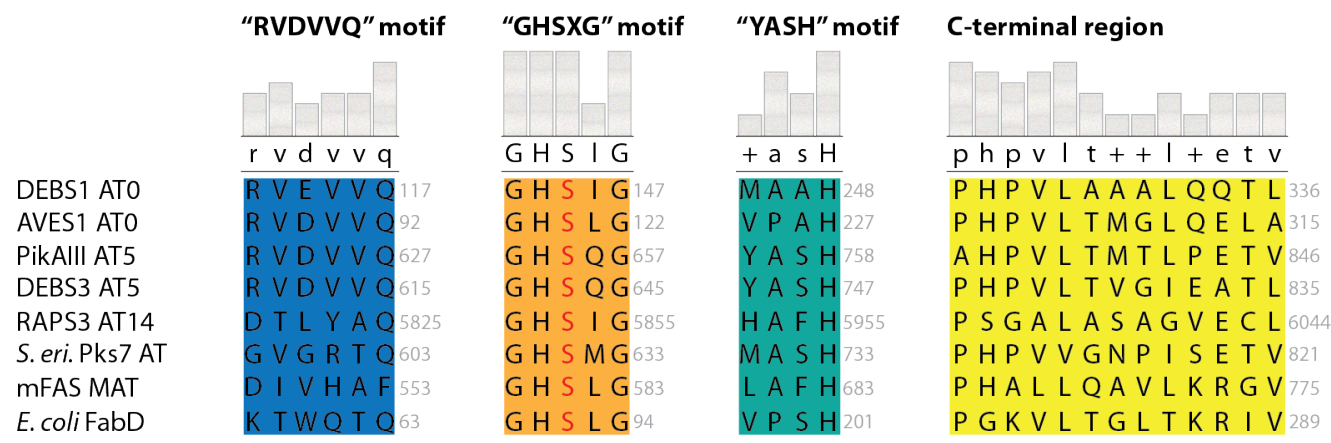

Figure 3.3: Excerpt of the sequence alignment of AT domains showing the characteristic motifs and regions for substrate specificity with the active site serine depicted in red. Primary sequences from priming ATs DEBS1 AT0 (UniProt: Q03131) and AVES1 AT0 (Q79ZN1), from specific elongation ATs PikAlll AT5(Q9ZGI3), DEBS3 AT5 (Q03133), RAPS3 AT14 (Q54299), and E. coli FabD (P0AA19), and from bifunctional ATs S. eri. Pks7 AT (A4FKH2) and mFAS MAT (P19096). Alignment created using CLC Main Workbench 6.9.1. 


\section{Results}

\subsubsection{Isolation of Acyl Transferases}

Recent functional and structural characterization revealed that type I FAS and PKS systems form stable KS-AT dimers. ${ }^{[9,99]}$ Therefore, all elongation AT domains were expressed as KS-AT didomains. A KS knockout, leading to constructs denoted as $\mathrm{KS}^{0}-\mathrm{AT}$, allowed for selectively inspecting the AT domain in transacylation properties. Priming AT domains and FabD were expressed as standalone proteins. Different DEBS1 AT0 constructs did not result in soluble protein. Thus, a construct with C-terminal maltose binding protein (MBP) fusion was designed. Recombinant expression of all ATs in E. coli gave sufficient yields of soluble, high-quality proteins (Tab.S1). Sodium dodecyl sulfate polyacrylamide gel electrophoresis (SDS-PAGE) confirmed high purity after tandem affinity chromatography (Fig. S1). Analytical size exclusion chromatography (SEC) (Fig. S2) revealed stable dimers for Pks7 and PikAllIM5 KS ${ }^{0}$-AT as well as tetrameric and dimeric oligomers for DEBS3M5 KS ${ }^{0}$-AT. Surprisingly, RAPS3M14 $\mathrm{KS}^{0}$-AT eluted from SEC mainly as monomer, besides a subfraction of tetrameric species. We note that the prevalence for a monomeric state should not impact AT activity, since the dimeric interface is formed by the KS domain, while the AT domain is monomeric in its active form. As expected, the standalone ATs E. coli FabD, DEBS1M0 AT-MBP, and AVES1M0 AT were solely monomeric. Thermal shift assay (TSA) in two buffers validated protein quality, giving melting points within a range of $45-65^{\circ} \mathrm{C}$ and $36-61{ }^{\circ} \mathrm{C}$ in storage and assay buffer, respectively (Tab. S2 and Fig. S3). All AT domains were prepared in biological triplicates, produced from single clones of one or more independent plasmid transformations.

\subsubsection{Isolation of Acyl Carrier Proteins}

Complete kinetic characterization of AT domains requires high yield and high quality of the corresponding ACPs. In order to generate holo-ACP, co-expression of ACPs with the 4'-PPT from Bacillus subtilis ( $\mathrm{Sfp}$ ) was performed, which, however, resulted in insufficient yields of most ACPs. Using codon-optimized sequences, the holo-ACP yields increased significantly. ACPs were purified by affinity chromatography and SEC of which the latter allowed selecting soluble ACPs. For typical expression yields and SDS-PAGE analyses, see Table S3 and Figure S4. Quantitative holo-ACP formation was confirmed by mass spectrometry (MS; Tab. S4 and Fig. S5). Protein integrity was confirmed by TSA giving melting temperatures within a range of $53-64{ }^{\circ} \mathrm{C}$ and $50-65^{\circ} \mathrm{C}$ in storage and assay buffer, respectively (Tab. S2 and Fig. S6). 


\subsubsection{Initial Substrate Screening}

At the outset of the functional characterization of AT domains, we performed an initial substrate screen. In doing so, three substrates were used for the elongation ATs: Mal and MMal, as typical elongation units, and Ac, as typical priming unit, and likewise a negative control for the ATs of modular PKSs. For the priming ATs, two priming substrates (the native and a non-native typical priming unit) and $\mathrm{Mal}$ as a negative control were used. For the initial substrate screen, AT domains were probed at fixed ACP and X-CoA concentrations.

With our initial screen, we essentially confirmed the substrate specificity of the elongation ATs from modular PKSs (DEBS3M5, PikAllIM5, and RAPS3M14) as expected from the chemical structure of the product (Fig. 3.4A and Tab. S5): The AT domains of DEBS3M5 and PikAllIM5 transferred solely MMal, and RAPS3M14 solely Mal. The AT domains of the iterative Pks7 tolerated MMal besides its natural elongation substrate $\mathrm{Mal}$, as well as the priming substrate $A c$ at slower rate. Since MMal-CoA is present in the native host S. erythraea, the loose specificity implies that both elongation substrates can be loaded for condensation. We analyzed Pks7 in transacylation of both substrates for deeper insight in the following (see section 3.3.6). For the mMAT, broad substrate tolerance was already shown previously, including the fast transfer of the priming substrate Ac and elongation substrate Mal with similar rates as well as the transfer of MMal at lower rate. [9] The E. coli AT FabD tolerated MMal in addition to its native elongation unit Mal, as published previously. [216]

Probing AT-mediated hydrolysis, we observed that most domains hydrolyze their native substrates with higher rates than non-native substrates (Fig. 3.4B). ${ }^{\vee}$ This overlapping substrate specificity of transacylation and hydrolysis was also reported before. ${ }^{[133,143]}$ Only PikAIII AT5 showed higher hydrolysis rates for the non-native elongation substrate Mal, which was also reported for PikAIV AT6. [132] Overall, this data confirms that there is no general hydrolysisbased proofreading mechanism present in AT domains for clearing the active site from wrongly loaded acyl moieties, as previously suggested. [217,218]

\footnotetext{
'We note that for determination of transacylation rates, self-acylation of ACP and not AT-mediated hydrolysis was subtracted as background. Accordingly, in this initial screening, the PKSs' transacylation rates may contain hydrolysis rates to some extent.
} 


\section{Results}

A

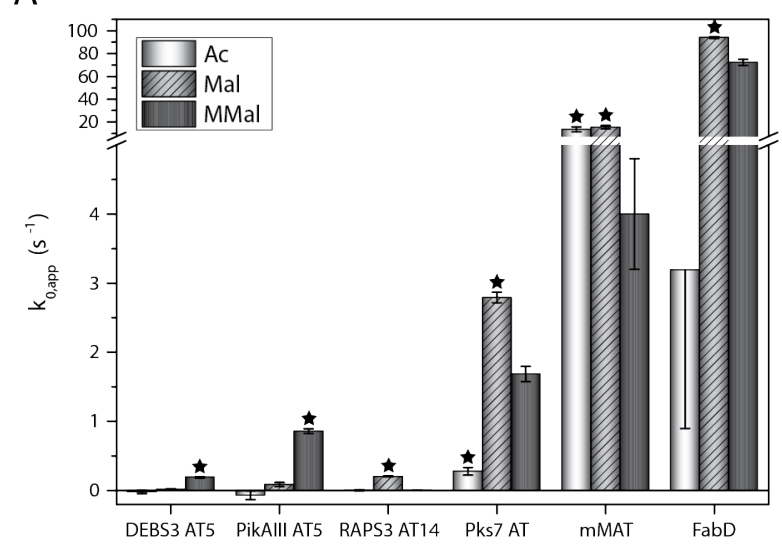

C

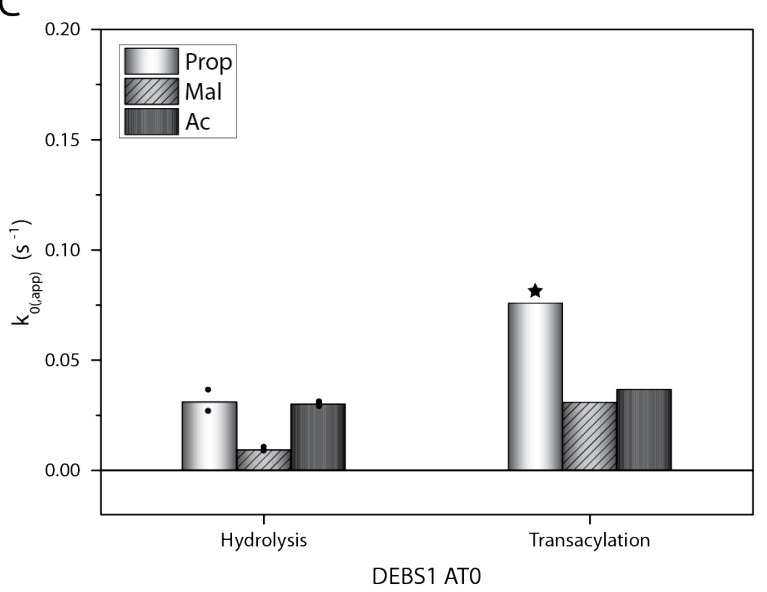

B

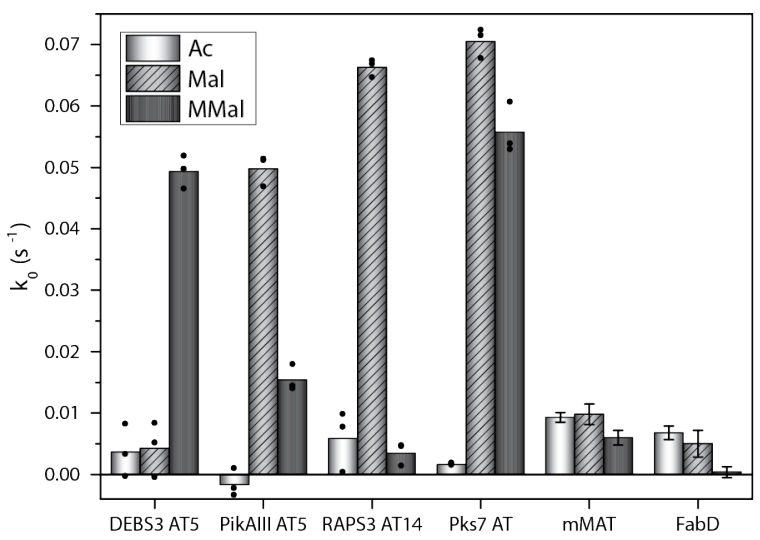

D

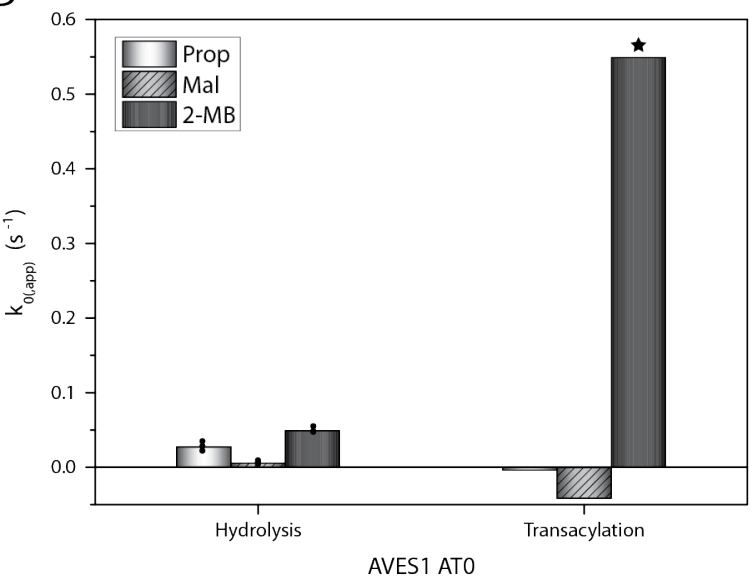

Figure 3.4: Initial screening of FAS and PKS systems. (A) Transacylation screening. Loading of Ac- (white), Mal- (light gray, hatched), and MMal-CoA (dark gray) was measured in presence of ACP. Substrate concentrations (X-CoA and ACP) were fixed at $50 \mu \mathrm{M}$. Bars indicate the average substrate turnover. A star indicates the native substrates for transacylation. Error bars show the standard deviation of biological triplicates. mMAT data from a previous study, referring to $k_{c a t}$, app at saturated $\mathrm{X}-\mathrm{CoA}$ concentration and $60 \mu \mathrm{M}$ ACP. [9] (B) Hydrolysis screening. Loading of Ac- (white), Mal- (light gray, hatched), and MMal-CoA (dark gray) was measured in absence of ACP. Substrate concentration (X-CoA) was fixed at $50 \mu \mathrm{M}$. Bars indicate the average substrate turnover. Black dots correspond to the average of technical triplicates of one biological replicate. Error bars show the standard deviation of biological triplicates. mMAT data from a previous study, referring to $k_{\text {cat }}$ at saturated $\mathrm{X}$-CoA concentration. ${ }^{[9]}$ (C) Initial hydrolysis and transacylation screening of DEBS1M0 AT. Loading of Prop- (white), Mal- (light gray, hatched), and Ac-CoA (dark gray) was measured in absence and presence of ACP, respectively. Substrate concentrations ( $\mathrm{X}-\mathrm{Co} A$ and ACP) were fixed at $50 \mu \mathrm{M}$. Hydrolysis bars indicate the average substrate turnover. Black dots correspond to the average of technical triplicates of one biological replicate. Transacylation bars are exemplary single well measurements. The star indicates the native substrate for transacylation. (D) Initial hydrolysis and transacylation screening of AVES1M0 AT. Loading of Prop- (white), Mal(light gray, hatched), and 2-MB-CoA (dark gray) was measured in absence and presence of ACP, respectively. Substrate concentrations (X-CoA and ACP) were fixed at $50 \mu \mathrm{M}$. Hydrolysis bars indicate the average substrate turnover. Black dots correspond to the average of technical triplicates of one biological replicate. Transacylation bars are exemplary single well measurements. The star indicates the native substrate for transacylation. 
We faced difficulties measuring the transacylation rates mediated by the priming AT of DEBS. Large signal fluctuations occured in numerous measurements indicating that there was no significant acyl transfer. Comparing hydrolysis rates and presumed transacylation rates confirmed this assumption: Transacylation rates were in the same range and not significantly higher compared to hydrolysis rates (Fig. 3.4C). The priming AT of AVES, on the other hand, showed significantly higher transacylation rates than hydrolysis rates like all the other systems analyzed (Fig. 3.4D). This indicates that the MBP fusion is interfering with the DEBS1M0 AT-ACP interaction. Nevertheless, the acyl substrate seemed to be loaded onto the AT domain, but transfer onto the ACP domain is not possible anymore. Both priming ATs accepted the native and the non-native priming substrate in hydrolysis.

Based on this initial screening, full kinetic analysis of AT-mediated hydrolysis and transacylation was performed with the substrates that are preferentially transferred. For the priming ATs, only AT-mediated hydrolysis was analyzed.

\subsubsection{AT-mediated Hydrolysis}

To analyze AT-mediated hydrolysis, the substrates $\mathrm{X}$-CoA were titrated in absence of ACP. We faced difficulties in conducting the hydrolysis assay, originating from extreme differences in $\mathrm{K}_{\mathrm{m}}^{\mathrm{X}-\mathrm{CoA}}$, and we were just able to determine kinetic parameters precisely for $\mathrm{K}_{\mathrm{m}}^{\mathrm{X}-\mathrm{Co}}{ }^{\mathrm{A}}$ values lying within suited conditions for recording Michaelis-Menten kinetics.

1. For high Michaelis-Menten constants, high substrate concentrations have to be used. This can lead to experimental problems caused for example by substrate inhibition effects, and only rough values for $\mathrm{K}_{\mathrm{m}}^{\mathrm{X}-\mathrm{CoA}}$ and $\mathrm{k}_{\text {cat }}$ can be given, leading also to catalytic efficiencies $k_{c a t} / K_{m}^{X-C o A}$ that are error-prone.

2. For low Michaelis-Menten constants $\mathrm{K}_{\mathrm{m}}^{\mathrm{X}-\mathrm{CoA}}$, low substrate concentrations have to be used, causing poor signal-to-noise ratios in the assay. Increasing enzyme concentrations leads to disregard of Michaelis-Menten concentration requirement. Thus, for AT domains with low $\mathrm{K}_{\mathrm{m}}^{\mathrm{X}-\mathrm{CoA}}$, just $\mathrm{k}_{\mathrm{cat}}$ is determined precisely, whereas $\mathrm{K}_{\mathrm{m}}^{\mathrm{X}-\mathrm{CoA}}$ and therefore also $\mathrm{k}_{\mathrm{cat}} / \mathrm{K}_{\mathrm{m}}^{\mathrm{X}-\mathrm{Co} \mathrm{A}}$ are approximate values. 
Table 3.1: Kinetic parameters determined for AT-mediated hydrolysis. Measurement performed in technical triplicates of biological triplicates (DEBS3 AT5, DEBS1 AT0 with Ac), technical triplicates of one replicate (PikAlll AT5, RAPS3 AT14, DEBS1 AT0 with Prop, and AVES1 AT0 with 2-MB and Prop), or biological triplicates (Pks7 with Mal and MMal). Abbreviations: n. d.: not determinable. [a] This system has a very low $\mathrm{K}_{m}^{\mathrm{X}-\mathrm{CoA}}$. Only $\mathrm{k}_{\mathrm{cat}}$ is determined precisely. $[\mathrm{b}]$ This system has a very high $\mathrm{K}_{\mathrm{m}}^{\mathrm{X}-\mathrm{CoA}}$. Measurement gave large signal fluctuations (Fig. S7). Corresponding kinetic values with substantial errors are indicated by constants in italic letters. [c] Measurement gave large signal fluctuations (Fig. S7). Corresponding kinetic values with substantial errors are indicated by constants in italic letters. [d] mMAT from previous study. [9]

\begin{tabular}{|c|c|c|c|}
\hline $\begin{array}{l}\text { Protein } \\
\text { X-CoA }\end{array}$ & $k_{\text {cat }}\left(s^{-1}\right)$ & $\mathrm{K}_{\mathrm{m}}^{\mathrm{X}-\mathrm{CoA}}(\mu \mathrm{M})$ & $\begin{array}{c}\mathrm{k}_{\text {cat }} / \mathrm{K}_{\mathrm{m}}^{\mathrm{X}-\mathrm{CoA}} \\
\left(\mathrm{s}^{-1} \mathrm{M}^{-1}\right)\end{array}$ \\
\hline $\begin{array}{l}\text { DEBS3 AT5 } \\
\text { MMal }\end{array}$ & $5.31 \times 10^{-2} \pm 9.62 \times 10^{-4}$ & $7.88 \pm 3.98 \times 10^{-1}$ & $6.7 \times 10^{3}$ \\
\hline $\begin{array}{l}\text { PikAlll AT5 } \\
\text { MMal[a] }\end{array}$ & $1.94 \times 10^{-2} \pm 5.03 \times 10^{-4}$ & $<8.91 \times 10^{-1}$ & $>2.2 \times 10^{4}$ \\
\hline $\begin{array}{l}\text { PikAIII AT5 } \\
\text { Mal }{ }^{[b]}\end{array}$ & $1.95 \times 10^{-1} \pm 1.54 \times 10^{-2}$ & $99.9 \pm 28.8$ & n. d. \\
\hline $\begin{array}{l}\text { RAPS3 AT14 } \\
\text { Mal[a] }\end{array}$ & $6.51 \times 10^{-2} \pm 1.89 \times 10^{-3}$ & $<5.45 \times 10^{-1}$ & $>1.2 \times 10^{5}$ \\
\hline $\begin{array}{l}\text { Pks7 AT } \\
\text { Mal[a] }\end{array}$ & $9.93 \times 10^{-2} \pm 3.17 \times 10^{-3}$ & $<4.74 \times 10^{-1}$ & $>2.1 \times 10^{5}$ \\
\hline $\begin{array}{l}\text { Pks7 AT } \\
\text { MMal[a] }\end{array}$ & $7.34 \times 10^{-2} \pm 1.51 \times 10^{-3}$ & $<3.98 \times 10^{-1}$ & $>1.8 \times 10^{5}$ \\
\hline $\begin{array}{l}\text { DEBS1 AT0 } \\
\text { Prop }\end{array}$ & $3.08 \times 10^{-2} \pm 2.37 \times 10^{-3}$ & $<5.46 \times 10^{-1}$ & $>5.6 \times 10^{4}$ \\
\hline $\begin{array}{l}\text { DEBS1 AT0 } \\
\text { Ac }\end{array}$ & $3.65 \times 10^{-2} \pm 1.65 \times 10^{-3}$ & $7.73 \pm 9.70 \times 10^{-1}$ & $4.7 \times 10^{3}$ \\
\hline $\begin{array}{l}\text { AVES1 AT0 } \\
\text { 2-MB }\end{array}$ & $4.86 \times 10^{-2} \pm 3.75 \times 10^{-4}$ & $<2.12 \times 10^{-1}$ & $>2.3 \times 10^{5}$ \\
\hline $\begin{array}{l}\text { AVES1 AT0 } \\
\text { Prop }\end{array}$ & $5.19 \times 10^{-1} \pm 1.08 \times 10^{-2}$ & $25.2 \pm 18.5$ & n.d. \\
\hline $\begin{array}{l}\text { mMAT } \\
\text { Mal }[\mathrm{d}]\end{array}$ & $(9.8 \pm 1.7) \times 10^{-3}$ & n. d. & n.d. \\
\hline $\begin{array}{l}\text { mMAT } \\
\text { MMal[d] }\end{array}$ & $(6.0 \pm 1.2) \times 10^{-3}$ & n. d. & n.d. \\
\hline $\begin{array}{l}\text { mMAT } \\
A_{c}^{[d]}\end{array}$ & $9.3 \times 10^{-3} \pm 1.2 \times 10^{-4}$ & n. d. & n.d. \\
\hline
\end{tabular}


Given these constraints, only DEBS3M5 (with MMal) and DEBS1M0 (with Ac) AT-mediated hydrolysis was eventually characterized in $\mathrm{K}_{\mathrm{m}}^{\mathrm{X}-\mathrm{CoA}}$ and $\mathrm{k}_{\mathrm{cat}}$ at high confidence, while for the other AT domains of PKSs partly just rough values were determined (Tab. 3.1). Kinetic parameters determined for DEBS3M5 AT-mediated hydrolysis are very similar to the data on DEBS2M3 AT-mediated hydrolysis previously published by Dunn et al. [133] Overall, ATmediated hydrolysis rates for the native substrates are similar for all analyzed PKS systems. Hydrolysis mediated by mMAT is very slow compared to the PKS systems. Hydrolysis by FabD was not measurable at all. For hydrolysis titration curves, see Figure $\mathrm{S} 7$.

\subsubsection{AT-mediated Transacylation}

To characterize the transacylation reaction, both substrates $\mathrm{X}-\mathrm{CoA}$ and ACP were titrated. Specifically, we titrated X-CoA substrates to five different, fixed ACP concentrations and globally fitted all titration curves. This approach is robust to measurement errors as well as delivers absolute kinetic constants and is in its quality superior to other approaches titrating one substrate while keeping the other at a fixed saturated concentration. ${ }^{[14,216]}$ For all ATs, absolute kinetic parameters were received, although the kinetic parameters for systems with high $\mathrm{K}_{\mathrm{m}}^{\mathrm{ACP}}$ were determined less accurately, simply because the vast amounts of ACP needed to cover an appropriate substrate range could not always be provided. This was particularly problematic for Pks7. Here, the ACP concentration was varied only within a range of $0.16-0.80 \times \mathrm{K}_{\mathrm{m}}^{\mathrm{ACP}}$ for the substrate MMal-CoA.

Overall, data reveals that PKS systems feature slower turnover rates than found in AT of FAS typel and type II (Tab.3.2 and Fig. 3.5), ${ }^{[9]}$ with the AT domain of the iterative Pks7 transferring the substrates with significantly higher rates than AT domains of modular PKSs. Catalytic efficiencies $\mathrm{k}_{\mathrm{cat}} / \mathrm{K}_{\mathrm{m}}^{\mathrm{X}-\mathrm{CoA}}$ and $\mathrm{k}_{\mathrm{cat}} / \mathrm{K}_{\mathrm{m}}^{\mathrm{ACP}}$ show that mMAT and FabD transacylate with highest efficiency, followed by the iterative Pks7 AT (about one order of magnitude less efficient than mMAT/FabD) and modular PKSs (another order of magnitude less efficient than Pks7 AT), indicating that the iterative systems feature low transition state energies for the transacylation reaction. Particularly, the catalytic efficiencies of the AT-acylating ping step $k_{c a t} / K_{m}^{X-C o A}$ is outstandingly high for mMAT and FabD compared to ATs of modular PKSs; e.g., Mal is transferred by mMAT and FabD with catalytic efficiency of $1.4 \times 10^{7} \mathrm{M}^{-1} \mathrm{~s}^{-1}$ compared to RAPS3 AT14 with $4.8 \times 10^{4} \mathrm{M}^{-1} \mathrm{~s}^{-1}$ (Tab. 3.2). 


\section{Results}

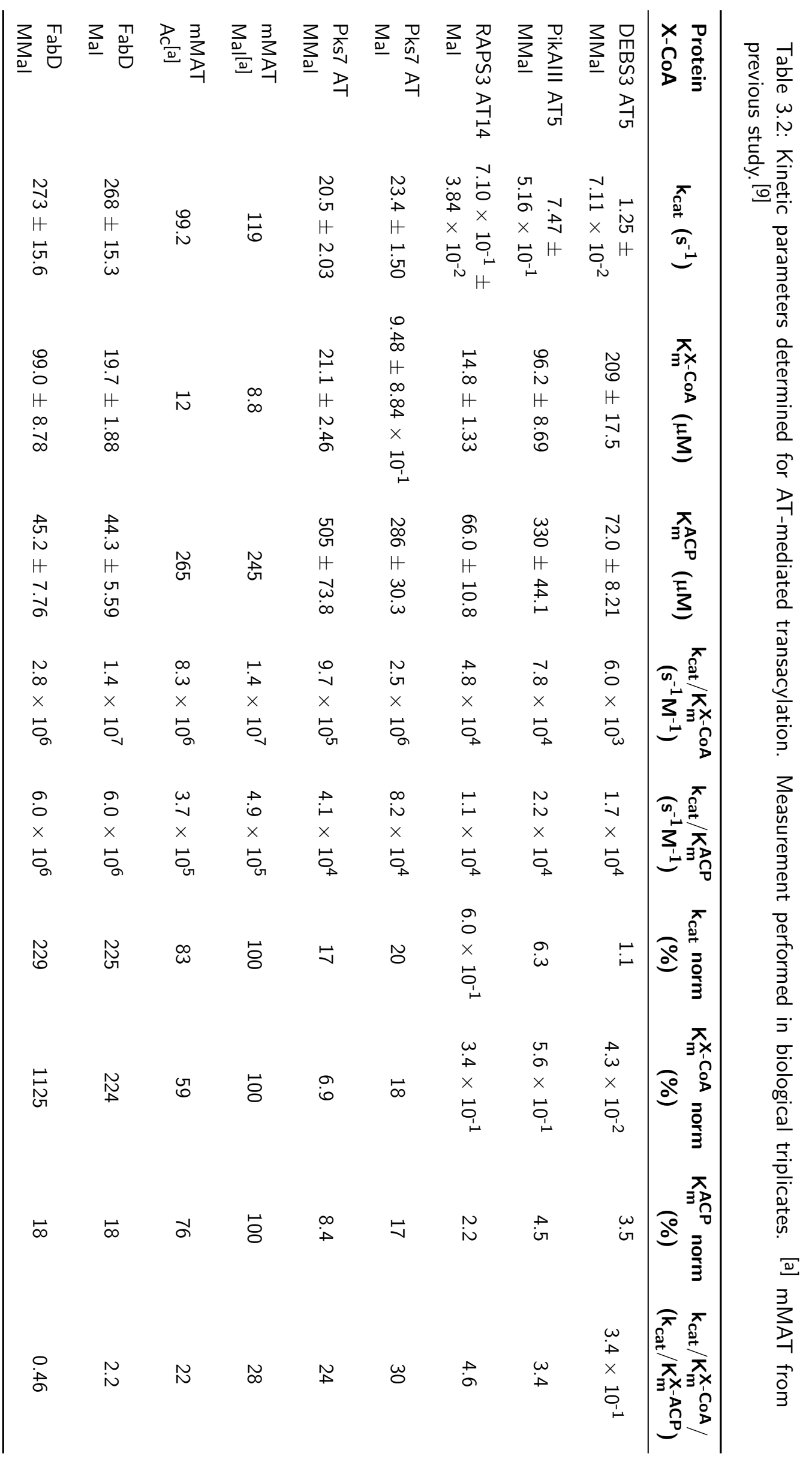


Comparing the AT-loading (ping) step with the ACP-loading (pong) step in catalytic efficiencies by forming the ratio $\left(\mathrm{k}_{\text {cat }} / \mathrm{K}_{\mathrm{m}}^{\mathrm{X}-\mathrm{CoA}}\right) /\left(\mathrm{k}_{\mathrm{cat}} / \mathrm{K}_{\mathrm{m}}^{\mathrm{ACP}}\right)$ shows that the iterative type $\mathrm{I}$ systems feature efficiency ratios of up to 30 for native elongation substrates, while ratios for the slower modular PKS systems are significantly lower (Tab.3.2). We interpret a high ratio as representing an AT domain that is particularly efficient in the initial acylation ping step (AT-X formation) and, in doing so, supports high overall transacylation rates due to a higher probability of a productive AT-X-ACP interaction in the pong step. Following this interpretation, the high catalytic efficiency of Pks7 AT and mMAT in their ping step is key to overall high turnover rates. Vice versa, the lower efficiency in the ping step of ATs of modular PKSs contributes to their slower transacylation rates. The type II FabD, on the other hand, shows an outstandingly high efficiency also for the pong step, which leads to a reduced ratio of efficiencies. This property might be explained by the dissociated enzymes in type II systems. Both substrates, acyl substrate and ACP, need to be selected out of the vast variety of molecules in the environment (efficient ping and pong step required). Whereas in type I systems, only the acyl substrate needs to be channeled into the assembly line (efficient ping step).

The $\mathrm{K}_{\mathrm{m}}^{\mathrm{X}-\mathrm{Co} A}$ values for $\mathrm{Ac}-\mathrm{Co} \mathrm{A}$ and $\mathrm{Mal}-\mathrm{CoA}$ are in the range of bacterial metabolite levels ${ }^{[219]}$ and are similar for most $A T$ substrate pairs. Interestingly, the $\mathrm{K}_{\mathrm{m}}^{\mathrm{MMal}-\mathrm{CoA}}\left(\mathrm{K}_{\mathrm{m}}^{\mathrm{X}-\mathrm{CoA}}\right.$ for MMal-CoA) values for DEBS3 AT5 and PikAlll AT5 are ten- to twentyfold higher than $\mathrm{K}_{\mathrm{m}}^{\mathrm{X}-\mathrm{CoA}}$ for other systems tested (Tab.3.2). This either indicates an adaptation to higher cellular MMal-CoA levels or a control mechanism for regulating MMal uptake depending on its availability. The regulation of polyketide biosynthesis by AT-mediated transacylation would be particularly efficient, when a limited metabolic flux of MMal into the polyketide biosynthetic pathway upon shortage of MMal-CoA (possible by the high $\mathrm{K}_{\mathrm{m}}^{\mathrm{MMal}-\mathrm{CoA}}$ ) becomes overall rate limiting. We note that a similar regulation of polyketide biosynthesis would not be plausible with Mal-CoA, which is dedicated mainly to the central metabolism of any bacterial cell and regulated in concentration to its need as precursor in fatty acid biosynthesis. ${ }^{[220]}$

Our data further shows that Michaelis-Menten constants $\mathrm{K}_{\mathrm{m}}^{\mathrm{ACP}}$ vary moderately for the different ATs (Tab. 3.2). Considering molar concentrations within the FAS/PKS compartment of about $1 \mathrm{mM}$ (rough calculation based on a cylinder volume of dimensions taken from MAS-like PKS; i. e., radius $10 \mathrm{~nm}$, height $15 \mathrm{~nm} ; 2$ molecules) as well as freely diffusing domains within the multidomain compartment, all AT domains of typel systems operate at high (Pks7) to saturated (other systems tested) ACP conditions so that the pong step runs at maximum rate $\left(v_{\max }\right)$. 


\section{Results}
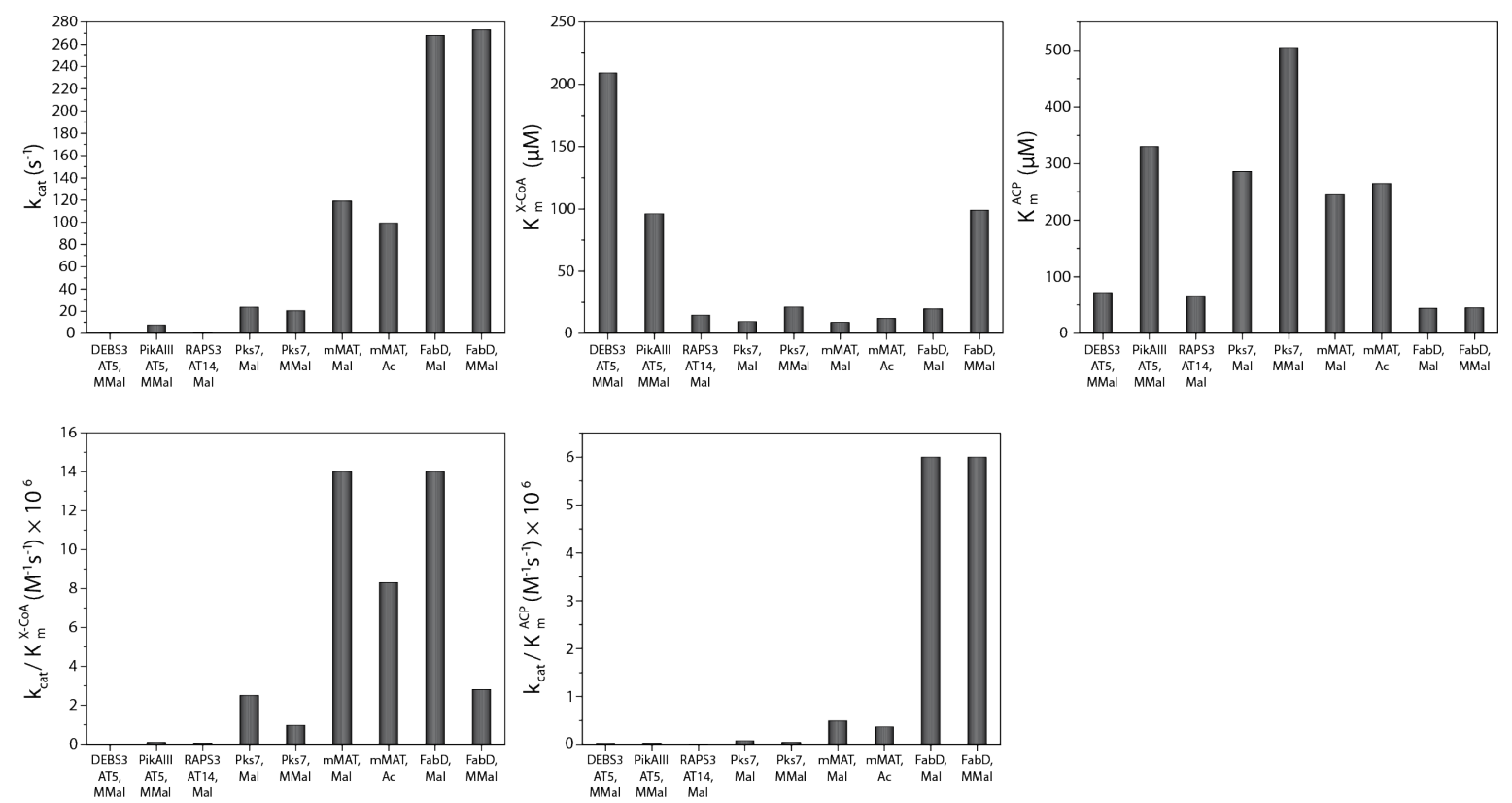

Figure 3.5: Plots of transacylation parameters. Turnover rate, Michaelis-Menten constants, and catalytic efficiencies determined for transacylation for the FAS and PKS systems. mMAT data from previous study. ${ }^{[9]}$

The analysis of the AT domain of the iterative Pks7 in transferring Mal and MMal gives further interesting insight into the AT-mediated transacylation. Both Michaelis-Menten constants $\mathrm{K}_{\mathrm{m}}^{\mathrm{X}-\mathrm{CoA}}$ and $\mathrm{K}_{\mathrm{m}}^{\mathrm{ACP}}$ differ for the two tested elongation substrates Mal-CoA and MMal-CoA (Tab. 3.2), which implies that the interaction of AT and ACP is modulated by the substrate to be transferred, similarly as recently suggested for E. coli type II FAS. ${ }^{[14]}$ Catalytic efficiencies differ for the two substrates, indicating a higher efficiency for Mal in both steps, the ATloading ping and the ACP-loading pong step. This data suggests a double selection for Mal over MMal to ensure the specific condensation with Mal in the organism. For transacylation titration curves, see Figure S8. We note that, based on the catalytic efficiency of the ping step, the Michaelis-Menten constants for the AT-mediated hydrolysis can be calculated (Tab. S6). 


\subsubsection{AT:ACP Interface Mutation Study}

Some of the molecular details to the ACP-mediated shuttling of substrates and intermediates to the catalytic domains in FASs and PKSs are being currently revealed. For example, interactions are guided by weak electrostatic interactions, ${ }^{[221]}$ and, as shown for the AT-ACP interaction in the E. coli typell system (FabD:AcpP interface), do not necessarily involve prevalent interfaces. ${ }^{14]}$ In order to help understanding this critical domain-domain interaction in type I complexes, the AT:ACP interface of DEBS3M5 was mutated and analyzed with the transacylation assay in enzyme kinetic detail. Three available AT:ACP complex structures $[125,126,159]$ were used for modeling the interaction (Fig. 3.6 and S9). Based on that, different bioinformatics tools were used to identify interfacial residues. Eventually two residues, A539 and R850, were selected that are likely involved in the AT-ACP interaction (residue numbering following UniProt entry Q03133). Overall, nine point mutations were introduced in the AT domain of the KS ${ }^{0}$-AT didomain construct of DEBS3M5; A539S, A539D, A539E, A539F, R850K, R850A, R850E, R850F, R850S. All mutants showed wild type-like properties during preparation (Tab.S7 and S8 and Fig. S10, S11, and S12).

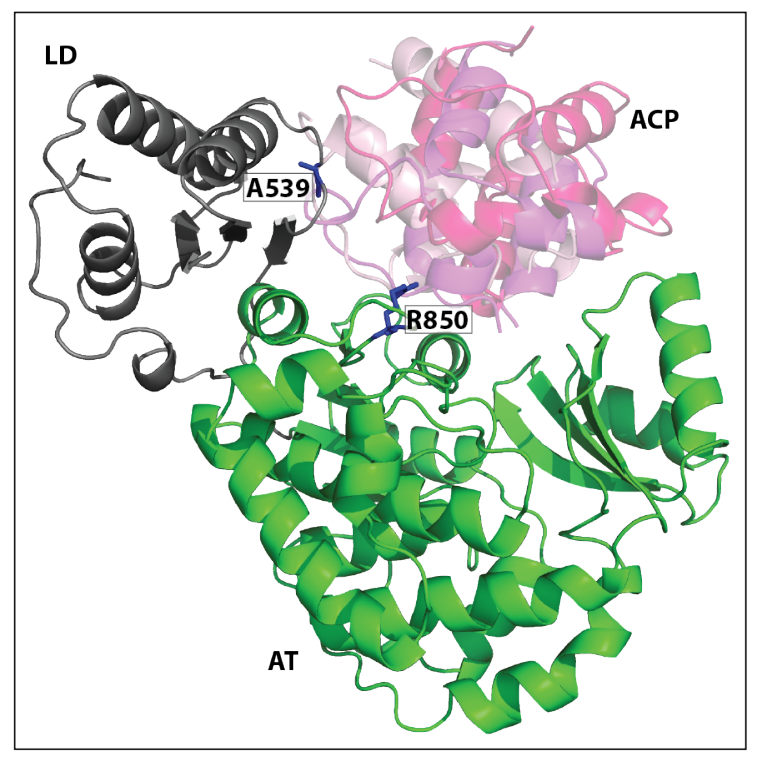

Figure 3.6: Cartoon representation of the modeled DEBS3M5 AT:ACP interface. AT domains

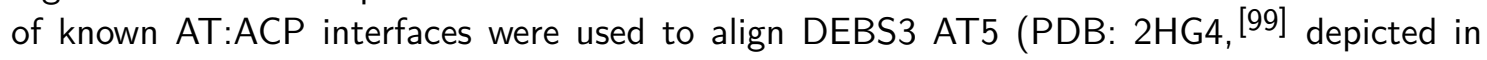
green) structurally. The LD in the KS-AT didomain substructure is depicted in gray. DSZS ACP (PDB: 5ZK4 ${ }^{[126]}$ ), VinL (PDB: 5CZD ${ }^{[125]}$ ), and hFAS ACP ${ }^{[159]}$ are depicted in light pink, hotpink, and magenta, respectively. Despite their differences, all models locate amino acids A539 and R850 (highlighted in blue) in the AT:ACP interfaces. Residue numbering following UniProt entry Q03133. 
In an initial screen, the mutants were tested in transacylation activity at fixed MMal-CoA and fixed ACP concentrations. We additionally screened the hydrolysis rates at fixed MMal-CoA concentrations. Since AT-mediated hydrolysis does not involve ACP, hydrolysis rates are able to report any non-local effect of the surface mutation. My initial screen revealed that some mutations affect both transacylation and hydrolysis, although the impact on hydrolysis was comparably lower than on transacylation (Fig. 3.7). This data shows that indeed some surface point mutations are invasive to structural and conformational properties that determine the enzymatic reaction. This phenomenon was pronounced for mutations introduced at R850, and not for A539, as depicted in Figure 3.7.

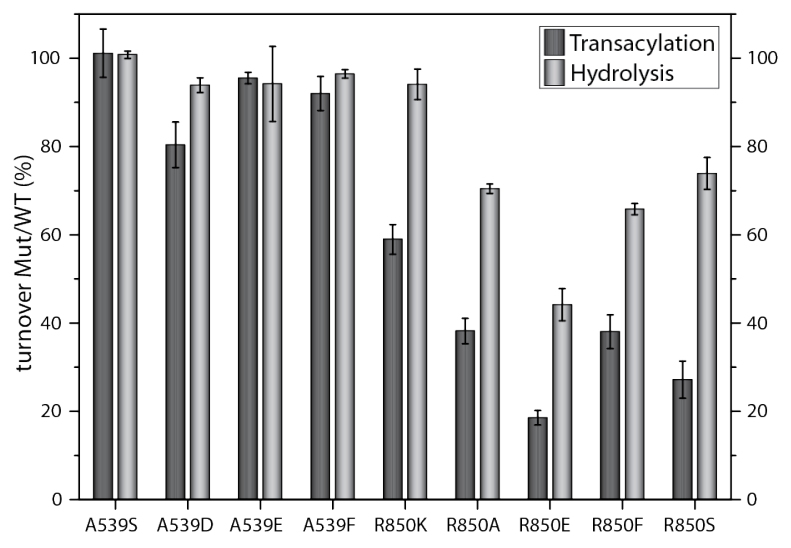

Figure 3.7: Transacylation (dark gray) and hydrolysis (light gray) screening of the different DEBS3M5 AT:ACP point mutants. The transacylation activity was measured in technical triplicates of one biological replicate; hydrolysis was measured in biological (A539E, R850K, R850E, R850S) or technical triplicates (A539S, A539D, A539F, R850A, R850F). The average activity of each mutant (Mut) is divided by wild type's (WT) activity and is given in \%. Error bars indicate the standard deviation of technical or biological triplicates. A539 mutants seem to have no influence on transacylation and hydrolysis, whereas R850 mutants decrease both transacylation and hydrolysis rates significantly.

Based on the initial screening, four mutations were chosen for full kinetic characterization to determine absolute kinetic constants; i.e., A539E, which showed nearly no effect in screening, and the mutations R850K, R850E, and R850S, which showed moderate to strong effects, preserving, inverting, and neutralizing the charge of the mutated residue. For protein quality control of proteins in biological replicates, see Table S8 and Figure S10, $\mathrm{S} 11$, and S12. We note that the ACP concentrations for the enzymatic analysis of mutant R850E were within a non-ideal range of $1-22 \times \mathrm{K}_{\mathrm{m}}^{\mathrm{ACP}}$ due to a low $\mathrm{K}_{\mathrm{m}}^{\mathrm{ACP}}$ value and the kinetic transacylation parameters for this mutant were just determined at lower confidence. 


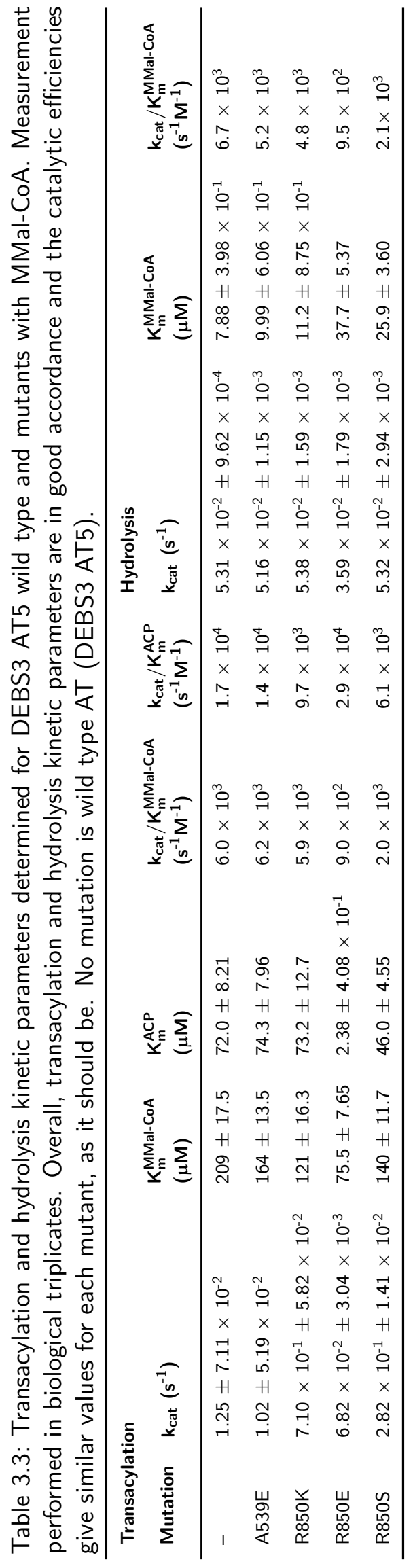




\section{Results}
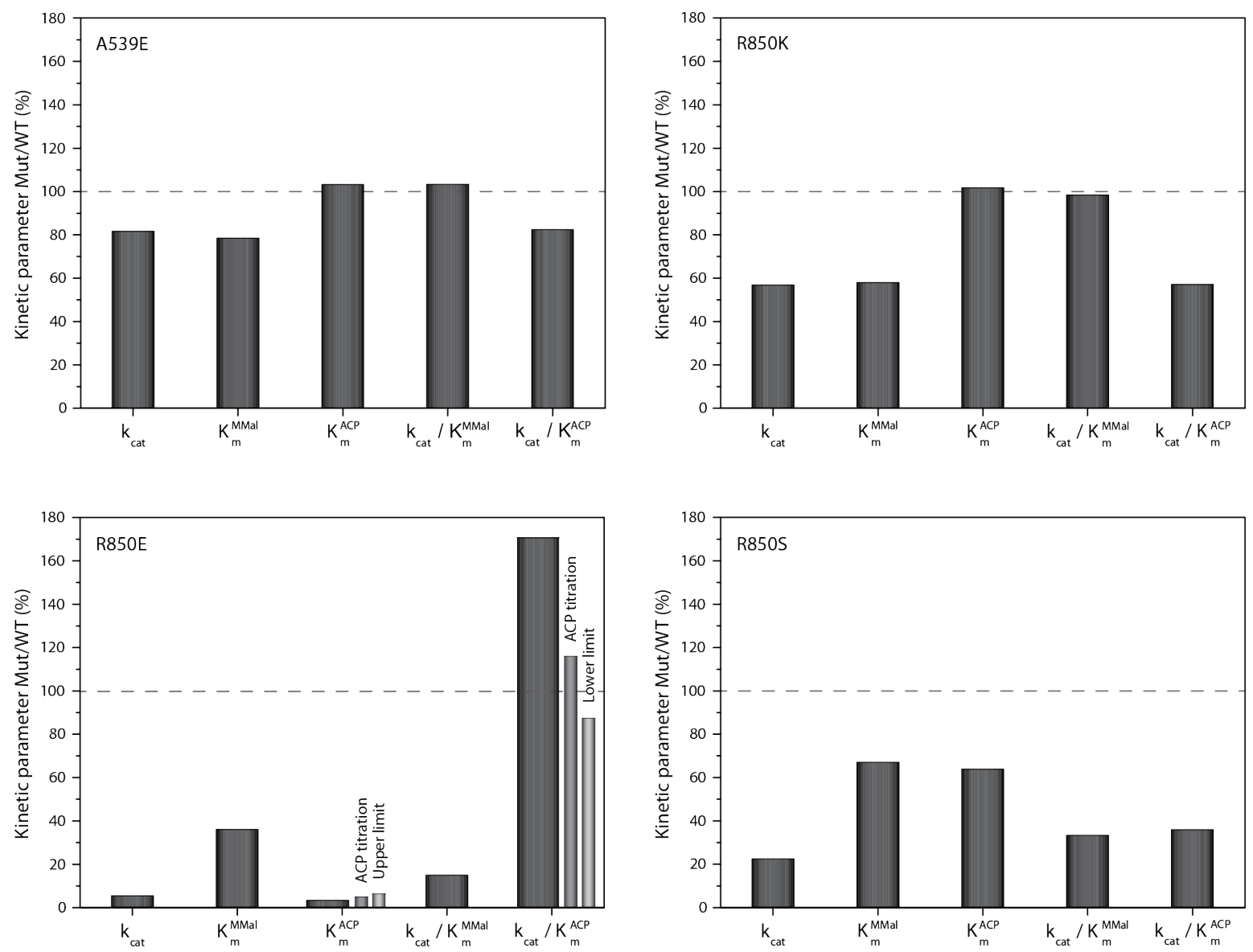

Figure 3.8: Ratio of kinetic constants determined for transacylation catalyzed by mutants (Mut) A539E, R850K, R850E, and R850S to wild type DEBS3M5 AT (WT) kinetic constants (in \%). R850E shows three ratios of $\mathrm{K}_{m}^{\mathrm{ACP}}$ Mut/WT corresponding to the different $\mathrm{K}_{\mathrm{m}}^{\mathrm{ACP}}$ received for this mutant: value determined via global fit (ratio shown in dark gray), determined via ACP titration without lowest ACP concentration (ratio shown in gray), and the upper limit of the value (ratio shown in light gray). Accordingly, this gives three corresponding ratios for the catalytic efficiency $k_{c a t} / K_{m}^{A C P}$. 
Trends in transacylation and hydrolysis turnover rates were in good accordance with the findings of the initial screening (Tab. 3.3 and Fig. 3.8). For hydrolysis and transacylation titration curves, see Figure S13 and S14. Mutant A539E showed comparable transacylation rates to the wild type protein ( $82 \%$ of wild type). Overall, kinetic characterization indicates that the site A539 is rather not involved in the AT-ACP and the AT-MMal-CoA interaction or mutations are not invasive to the AT-ACP interaction. The kinetics of the transacylation reaction is essentially unaltered compared to the wild type, as can also be seen in transition state energies for the ping step and pong step remaining at wild type level (Fig. 3.9). In contrast, R850 mutants were significantly compromised in transacylation activity (R850K with $57 \%$ of wild type, R850E 5.5\%, and R850S 23\%). vi Hydrolysis rates remained unaltered by the mutations A539E, R850K, and R850S, but dropped to $68 \%$ of wild type rates for mutation R850E. We note (again) that the transacylation rate always include the hydrolysis rate to some extent.

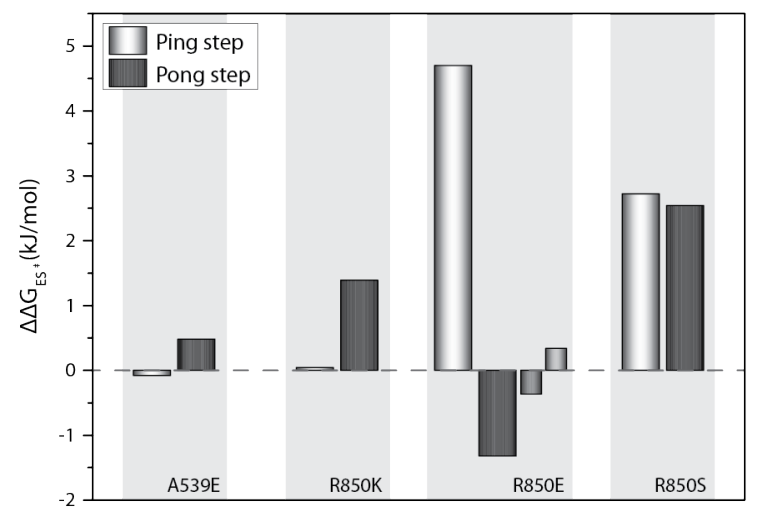

Figure 3.9: Difference in transition state energy (in $\mathrm{kJ} / \mathrm{mol}$ ) between mutants A539E, R850K, R850E, and R850S and wild type determined for the ping step (white bars) and the pong step (dark gray bars) of transacylation. Mutant R850E gives three values for $\mathrm{K}_{\mathrm{m}}^{\mathrm{ACP}}$ : one determined via global fit, one via ACP titration (gray), and the upper limit (light gray). Differences in transition state energy to wild type are shown in dark gray, gray, and light gray, respectively. $\Delta \Delta \mathrm{G}_{\mathrm{ES}^{\ddagger}}=-\mathrm{RT} \ln \left[\frac{\left(\mathrm{k}_{\mathrm{cat}} / \mathrm{K}_{\mathrm{m}}\right)^{\mathrm{Mut}}}{\left(\mathrm{k}_{\mathrm{cat}} / \mathrm{K}_{\mathrm{m}}\right)^{\mathrm{WT}}}\right]$ with the gas constant $\mathrm{R}, \mathrm{T}=298 \mathrm{~K}$ and the ratio of catalytic efficiencies between mutant (Mut) and wild type (WT).

${ }^{v i}$ For R850E, kinetic parameters of the pong step were only collected with lower confidence due to the low $\mathrm{K}_{\mathrm{m}}^{\mathrm{ACP}}$. For a reliable estimate on $\mathrm{K}_{\mathrm{m}}^{\mathrm{ACP}}$, we extracted data for saturated substrate MMal-CoA concentration titrated with $\mathrm{ACP}$ and received a $\mathrm{K}_{\mathrm{m}}^{\mathrm{ACP}}$ of $3.46 \mu \mathrm{M}$ and an upper boundary of $4.6 \mu \mathrm{M}$ (Fig. S15). 


\section{Results}

\subsection{Generation of a FAS-Based Chimeric Elongation Module}

Based on kinetic analysis of ATs, we selected ATs for construction of a FAS-based elongation module via domain swapping. The most important criterion was the strict specificity for a single elongation substrate. Only the three ATs from modular PKSs fulfill this requirement. For more variability, we aimed to design two elongation modules that selectively load Mal and MMal, respectively. Among the three elongation ATs from modular PKSs, only RAPS3 AT14 transfers Mal. For the MMal-specific AT, we had to decide between DEBS3 AT5 and PikAlll AT5. The advantage of the available crystal structure of the first outweighs the faster transacylation rates of the latter. Thus, in a first domain swapping attempt, we used RAPS3 AT14 and DEBS3 AT5.

\subsubsection{Chimeric mFAS with Swapped DEBS AT5 and RAPS AT14}

To identify proper domain boundaries for the AT swap, we used the crystal structures of mFAS and DEBS3M5 KS-AT (Fig. 3.10A and B) and created a structural alignment. Additionally, we created a sequence alignment to analyze and consider conserved areas and to transfer structural insights on RAPS3 AT14, for which no structural information is available. DEBS3M5 and RAPS3M14 KS-AT have high sequence identities of $31 \%$ and $30 \%$ with mFAS KS-AT, respectively. DEBS3M5 and RAPS3M14 KS-AT exhibit an even higher sequence identity of $48 \%$.

As described before, KS and AT are connected via an LD (see section 2.6.4). Both analyzed didomain structures are remarkably similar, although the overall structural alignment gives a high root-mean-square deviation (RMSD) of $4.824 \AA$ (721 atoms). Alignment of solely the KS and solely the AT domains reveals the high similarity of the single domains, giving an RMSD of $1.221 \AA$ (318 atoms) and $1.428 \AA$ (243 atoms), respectively (Fig. 3.10C and D). Besides, also the orientation of the AT towards the KS is almost the same. In contrast, the LD differs strongly in both structures. Structural alignment (not shown) results in a rotation of around $120^{\circ}$ and an RMSD of $10.111 \AA$ (41 atoms). The overall high similarity of the condensing part in both megasynthases is promising for the domain swapping approach. 


\section{Results}

A

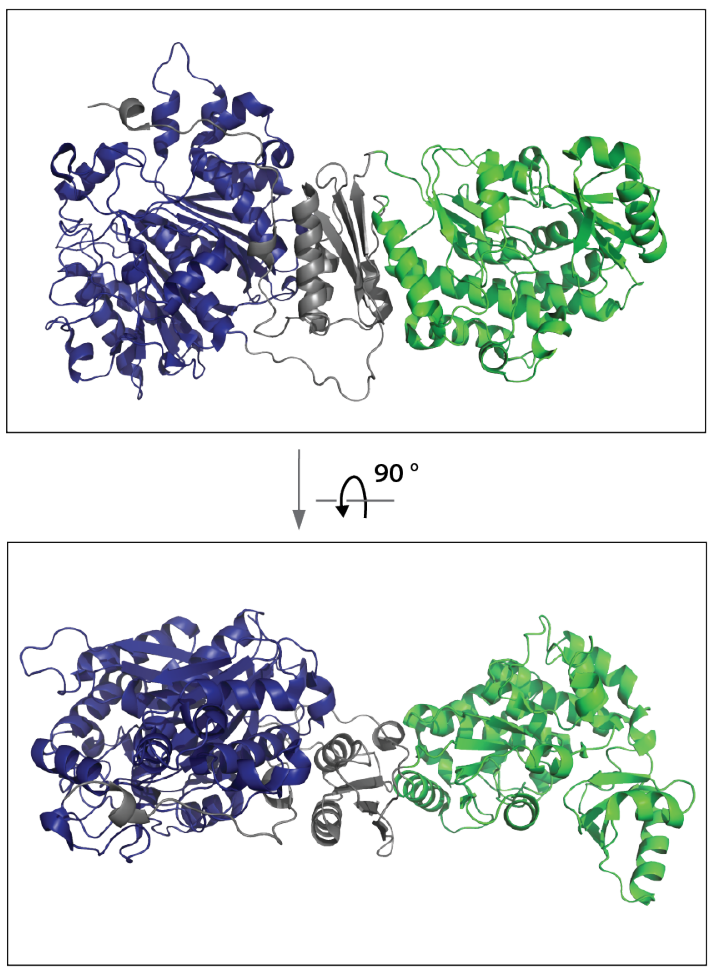

C

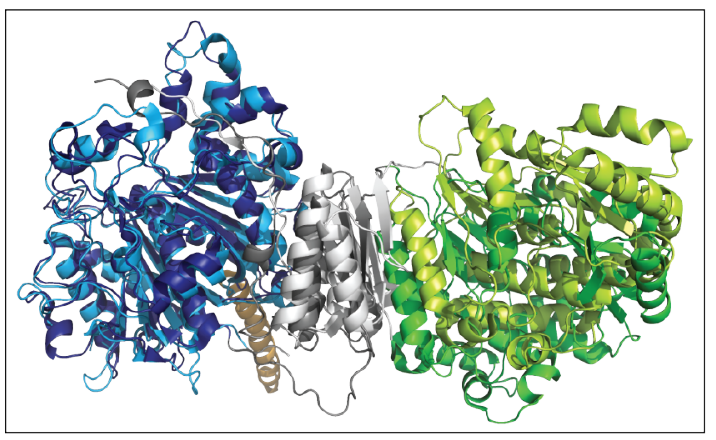

B

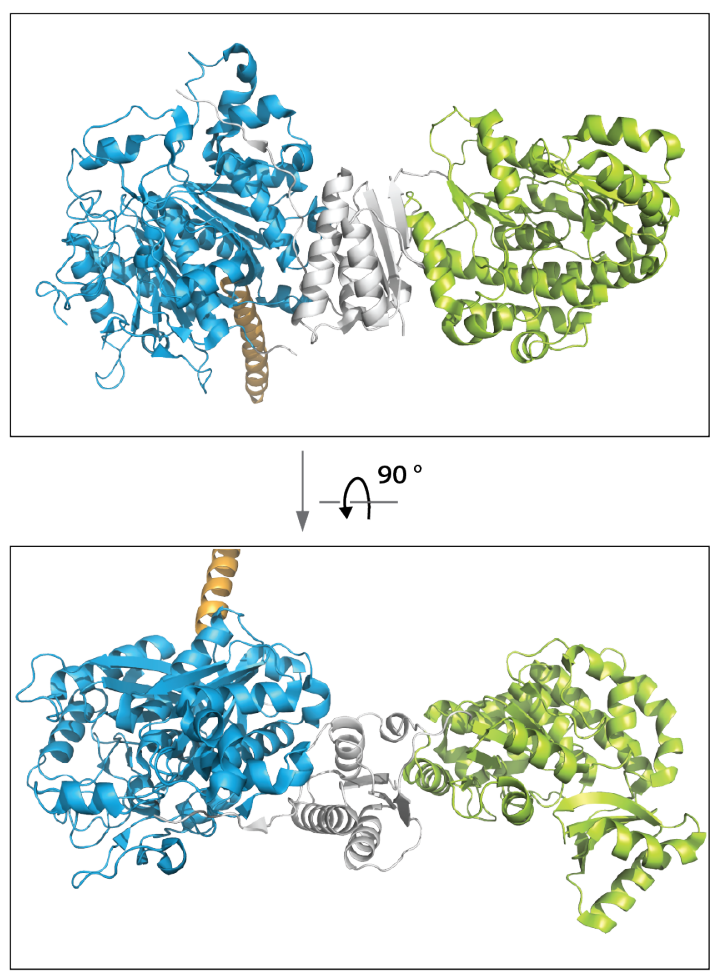

D

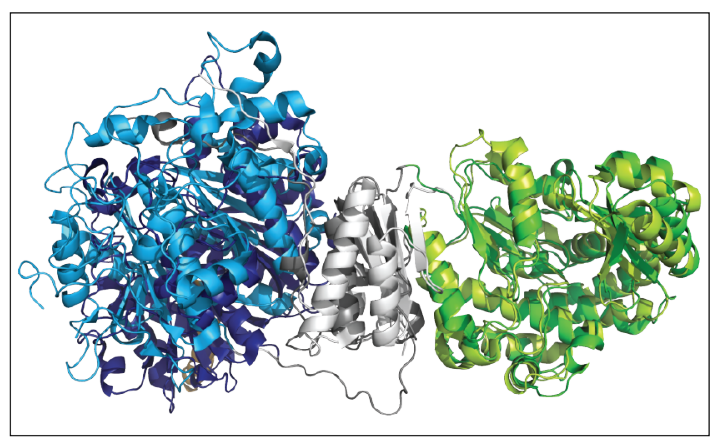

Figure 3.10: Structural similarity between the KS-AT didomain from mFAS (PDB: $5 M Y 0^{[9]}$ ) and DEBS3M5 (PDB: $2 \mathrm{HG}_{4}{ }^{[99]}$ ). (A) Cartoon representation of the KS-AT didomain from mFAS linked by the LD. KS, LD, and AT shown in dark blue, dark gray, and green, respectively. (B) Cartoon representation of the KS-AT didomain from DEBS3M5 linked by the LD. KS, LD, and AT depicted in light blue, light gray, and lemon, respectively. (C) Structural alignment of solely the KS domains reveals their high similarity (RMSD $=1.221 \AA$ ). mFAS and DEBS3M5 KS-AT shown in dark and bright colors, respectively. (D) Structural alignment of solely the AT domains shows their high resemblance $($ RMSD $=1.428 \AA$ ). mFAS and DEBS3M5 KS-AT shown in dark and bright colors, respectively. 


\section{Results}

Regarding the structures of the KS-AT didomains, one can identify two possible interfaces for an AT swap: The first approach exchanges solely the AT domain, creating an artificial LD:AT interface (boundaries between KAL and AT and between AT and PAL). The second strategy is to swap the AT domain with its LD, resulting in a new KS:LD interface (boundaries between $\mathrm{KS}$ and $\mathrm{KAL}$ and within $\mathrm{PAL}$ ). A recent study showed, that the interface between the $\mathrm{KS}$ and LD is better suited for AT swapping approaches. ${ }^{20]}$ However, every system has its own characteristic properties and might respond differently to changes in certain interfaces. Thus, we decided to design constructs with swapped AT and with swapped LD-AT, based on the structural alignment and the sequence alignment of the three KS-AT didomains (Fig. 3.11). For the AT swap, we chose the same domain boundaries based on both alignment types. For the LD-AT swap, the boundaries we chose based on the structure and sequence alignment differed considerably in the second domain boundary within the PAL (PKS PAL:mFAS PAL). Hence, it seemed reasonable to design two different constructs with modified boundaries - one following the structural alignment, one following the sequence alignment. 
A

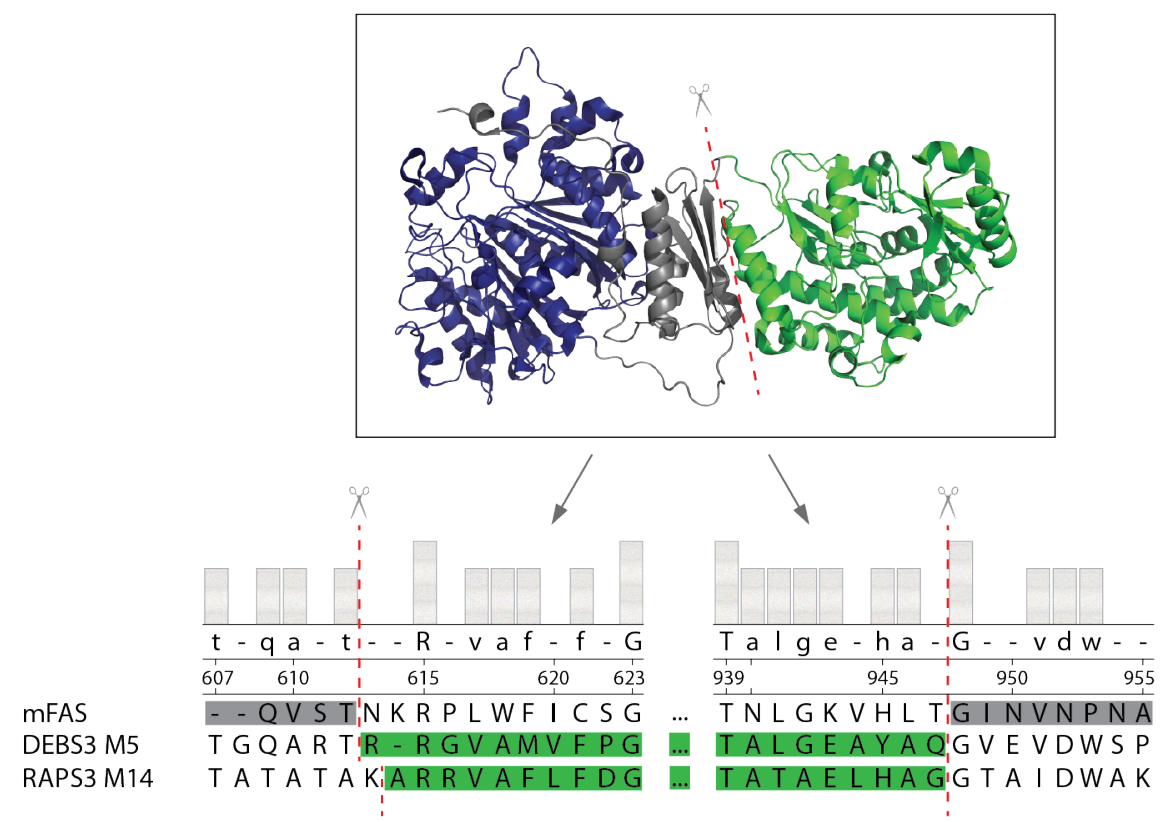

B

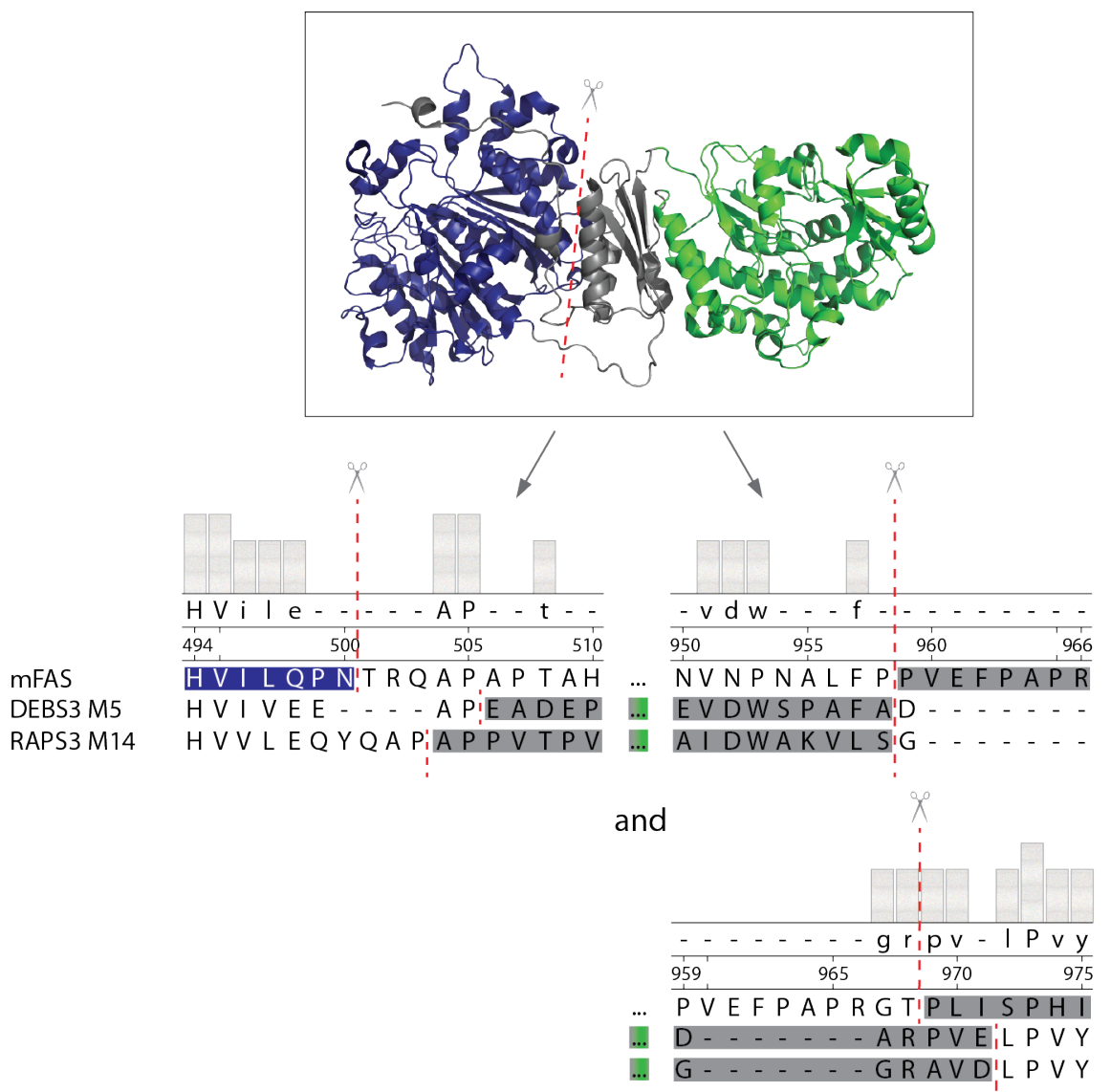

Figure 3.11: Domain boundaries for the AT swapping in mFAS. Cartoon representation of mFAS (PDB: $5 \mathrm{MYO}^{[9]}$ ) schematically shows the artificial interfaces introduced in domain swapping. KS, LD, and AT depicted in blue, gray, and green, respectively. Excerpt of alignment depicts the exact artificial domain boundaries. (A) Domain boundaries for the exchange of the AT domain in mFAS with DEBS3 AT5 and RAPS3 AT14. Primary sequences of KS-AT wild types show boundaries between KAL 
and AT as well as between AT and PAL. (B) Domain boundaries for the exchange of the LD-AT domain in mFAS with DEBS3 (LD-AT)5 and RAPS3 (LD-AT)14. Primary sequences of KS-AT wild types show boundaries between $\mathrm{KS}$ and $\mathrm{KAL}$ as well as within the PAL. Two alternative boundaries were chosen for the latter - the upper boundaries are based on the sequence alignment, the lower ones on the structure alignment.

Table 3.4: Test expression yields of chimeric mFAS with DEBS3 AT5 and RAPS3 AT14 after tandem affinity chromatography of $1 \mathrm{~L}$ expression culture.

\begin{tabular}{lccc}
\hline Domain Swapped & Pellet's Weight $(\mathbf{g})$ & Total Yield $(\boldsymbol{\mu g})$ & Yield $(\boldsymbol{\mu g})$ per g Pellet \\
\hline DEBS AT & 10.9 & 286 & 26.2 \\
DEBS (LD-AT)1 & 10.3 & 16.6 & 1.61 \\
DEBS (LD-AT)2 & 10.4 & 79.9 & 7.68 \\
RAPS AT & 12.0 & 56.4 & 4.70 \\
RAPS (LD-AT)1 & 10.8 & 14.0 & 1.30 \\
RAPS (LD-AT)2 & 15.2 & 86.6 & 5.70 \\
\hline
\end{tabular}

The chimeric constructs were expressed in E. coli and purified by tandem affinity chromatography. All proteins showed drastically reduced yields (Tab. 3.4) compared to the wild type mFAS (wild type yield around $1-2 \mathrm{mg} / \mathrm{L}^{[17,222]}$ ). Proteins were analyzed by SDS-PAGE (Fig. 3.12A). The first construct with swapped LD-AT from DEBS3 AT5 could not be isolated at all in full-length (exp. mass of $277 \mathrm{kDa}$ ), but showed some other protein bands most likely due to protein degradation. All other proteins were successfully isolated (exp. masses of 276-278 kDa) as indicated by the protein bands around $280 \mathrm{kDa}$. All elution fractions contained other bands of lower molecular weight proteins. Since purification via tandem affinity chromatography usually results in pure protein, ${ }^{[1,17]}$ these are most likely degraded proteins. The band of $170 \mathrm{kDa}$ could correspond to the protein without KS-AT, the band at $60 \mathrm{kDa}$ to KS-LD, and the band of $45 \mathrm{kDa}$ to KS or LD-AT domains. Western blot analysis could be used to give additional information. The construct with swapped DEBS3 AT5 showed an additional protein band of around $100 \mathrm{kDa}$ after the second affinity chromatography, which was not present in the elution fraction of the first (Fig. S16). This was definitely old protein bound to the second column and most probably a KS-AT construct. Therefore, the protein yield for this chimeric construct could not be determined precisely, but should lie within the double-digit $\mu g$ range. 
Even though the chimeras were only isolated once, I observe the following: Proteins with swapped LD-AT seem to demonstrate the value of structural information in domain swapping approaches. Constructs designed on the basis of the structural alignment gave higher yields than constructs with domain boundaries chosen based on the sequence alignment.

A

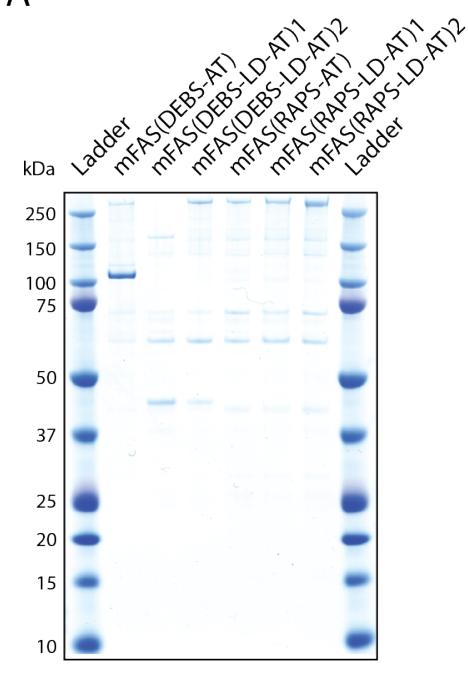

B

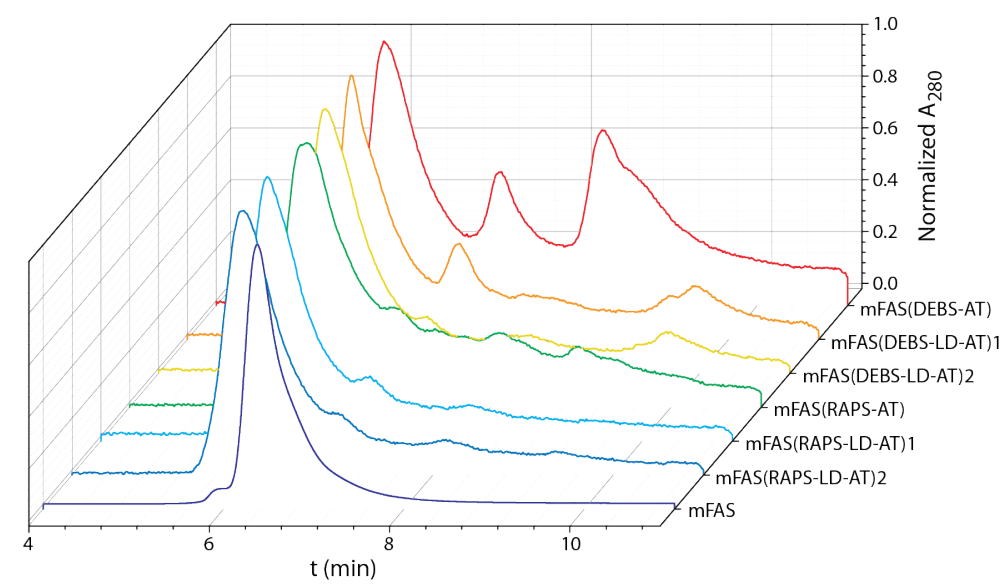

Figure 3.12: Analysis of chimeric mFAS constructs. (A) SDS-PAGE (NuPAGE Bis-Tris 4-12\%) of chimeric full-length mFAS. Elution fractions after tandem affinity chromatography. (B) Normalized size exclusion chromatogram of wild type mFAS and chimeric constructs.

Proteins were further analyzed regarding their oligomeric states via high performance liquid chromatography (HPLC)-SEC (Fig. 3.12B). The wild type mFAS solely formed dimeric species. The chimeric constructs, however, showed mainly higher oligomeric states and only partly dimeric states. Impurities of lower molecular weight proteins and degradation were observed in their chromatograms.

Overall, this first attempt to create a chimeric mFAS module illustrates the complexity of domain swapping and shows that fine-tuning might be needed to gain a functional protein in good yield and quality. Thus, we decided to focus on only one construct - the domain swapping with the AT domain from DEBS3M5, since structural information is available. Furthermore, we decided to reduce the complexity by working first on the didomain construct KS-AT, which is easier to modify, isolate, and analyze. After successful design of a stable chimeric didomain, this construct can be expanded to the full-length mFAS elongation module. 


\section{Results}

\subsubsection{Generation of mFAS/DEBS3M5 KS-AT Chimeras}

Analogously to the full-length chimeras, two chimeric KS-AT didomains were designed consisting of mFAS KS-LD with DEBS3 AT5 (FS37) and mFAS KS with DEBS3 (LD-AT)5 (FS38), respectively. Since the first LD-AT swap was not successful in full-length mFAS, only the second domain boundaries were used. It became obvious, that slight changes in domain boundaries can totally change the outcome of the chimera. Thus, we chose to design alternative constructs. The two mentioned chimeras (FS37 and FS38) served as starting point for

the design of six others. Following Yuzawa et al.'s strategy to design active chimeras, ${ }^{[20]}$ we modified the domain boundaries as follows, which is described schematically in Figure 3.13A:

1. One approach modifies the second boundary and keeps (only) the first part of the DEBS PAL (PAL1). In the AT only swap, this leads to a slightly shortened mFAS PAL and addition of the DEBS PAL1 (FS57). In the AT-LD swap, this involves a slightly lengthened mFAS PAL and a shortened DEBS PAL (FS60).

2. The second approach consists of modifying the first boundary. For the AT only swap, the mFAS KAL was slightly lengthened (FS58). For the LD-AT swap, the DEBS KAL was slightly lengthened (FS61).

3. The third approach is the combination of both (FS59 and FS62).

Sequence information of modified domain boundaries is shown in Figure 3.13B. 


\section{Results}

A

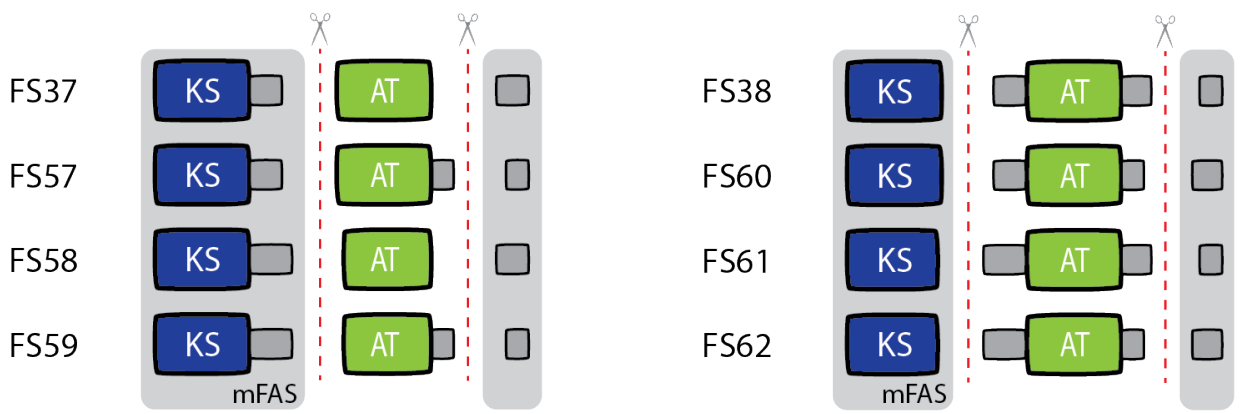

B
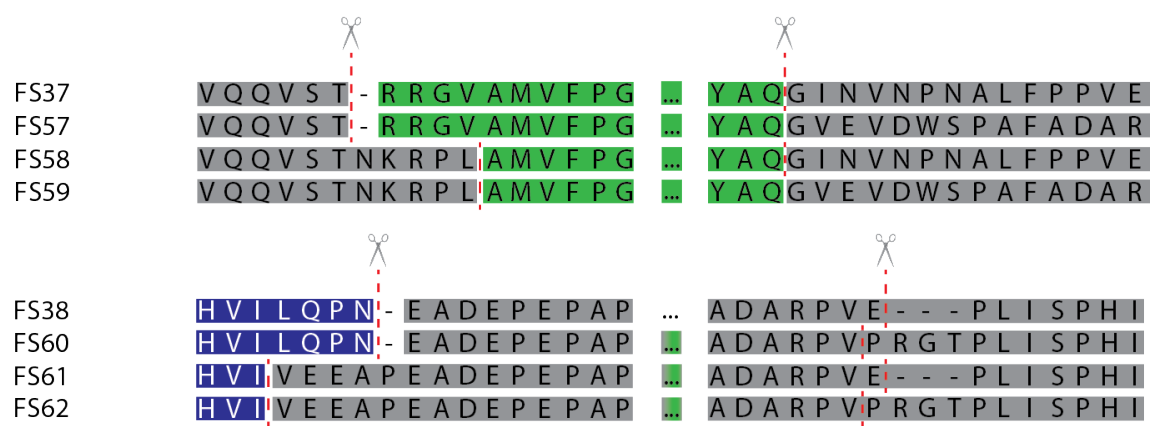

Figure 3.13: Design of mFAS/DEBS3M5 KS-AT chimeras. (A) Schematic representation of the KS-AT chimeras with modified domain boundaries. The linkers KAL and PAL are adapted in length to optimize the artificial interactions. (B) Domain boundaries in the KS-AT chimeras. Excerpt of the primary sequence of KS-AT chimeras shows the variation in linker regions.

Cloning, expression, purification, and analysis of chimeras were performed by Maren Berlinghof in the course of her master's thesis under my supervision. ${ }^{223]}$ All eight chimeras were successfully isolated, but yields were drastically reduced compared to wild type yields (Tab. 3.5, mFAS KS-AT $16 \mathrm{mg} / \mathrm{L}$, DEBS3M5 KS-AT $14 \mathrm{mg} / \mathrm{L}^{[1]}$ ). Analytical SDS-PAGE confirmed successful isolation of all chimeras (Fig. 3.14), but also showed some degraded proteins similar to the chimeric full-length mFAS. The band of $60 \mathrm{kDa}$ could correspond to KS-LD and the band of $45 \mathrm{kDa}$ could correspond to $\mathrm{KS}$. Judging from this test expression, domain swapping of AT with LD seemed to be more effective than solely swapping the AT domain, which is in agreement to Yuzawa et al.'s findings. ${ }^{20]}$ In case of the AT only swap, keeping the first part of PAL from the AT system did not improve expression yields and indicates that there are almost no important stabilizing interactions between AT and PAL. In case of LD-AT swap, the yield was significantly reduced by shortening the PAL to PAL1 from the AT system. This indicates that there are also important interactions between LD and PAL2. In case of single AT swapping, the modified domain boundaries between LD and AT seemed to be better suited, but conclusions cannot be drawn without uncertainty due to only single protein preparations and to the low significance of yields. In case of LD-AT swapping, the original boundaries between KS and LD seemed to be best suited. 


\section{Results}

Table 3.5: Test expression yields of KS-AT chimeras after tandem affinity chromatography of $1 \mathrm{~L}$ expression culture.

\begin{tabular}{lccc}
\hline Protein & Pellet's Weight $(\mathbf{g})$ & Total Yield $(\boldsymbol{\mu g})$ & Yield $(\boldsymbol{\mu g})$ per g Pellet \\
\hline FS37 & 8.11 & 73.3 & 9.04 \\
FS57 & 8.62 & 89.1 & 10.3 \\
FS58 & 7.60 & 131 & 17.2 \\
FS59 & 7.54 & 106 & 14.0 \\
\hline FS38 & 8.11 & 506 & 62.4 \\
FS60 & 6.82 & 101 & 14.8 \\
FS61 & 6.36 & 164 & 25.8 \\
FS62 & 7.58 & 171 & 22.6 \\
\hline
\end{tabular}

Without further purification, proteins were analyzed via TSA. Impurities may have affect the outcome, but trends in protein stability were still observed. Due to low yields, only the proteins FS37, FS38, FS58, and FS59 resulted in significant and analyzable melting curves (Fig. 3.14B). The fluorescence signal of all chimeras was already high from the start, indicating unfolded or degraded protein. ${ }^{[224]}$ Surprisingly, FS37 seemed to be significantly more stable than even the wild type proteins according to the determined melting temperatures. This can be explained by the fact that protein degradation may lead to stable fragments. ${ }^{225]}$ Interestingly, the other chimeric proteins seemed to reflect the wild type with higher portion of composition. FS58 and FS59 with solely AT exchanged consist mainly of mFAS parts. Both proteins showed similar melting behavior as the wild type mFAS KS-AT. FS38, on the other hand, with LD-AT from DEBS3M5 resembled its wild type behavior. More data on the melting behavior of the other chimeras would be preferable in order to evaluate if this is an actual consistent trend.

Furthermore, proteins were analyzed in their oligomeric states via HPLC-SEC (Fig. 3.14C and D). Due to low yields, only a limited amount of protein could be analyzed leading to a suboptimal signal-to-noise ratio for proteins FS57 and FS60-FS62. For these chimeras, mainly aggregates and higher oligomeric states were detected, besides the desired dimeric state. FS37 and FS38 showed aggregates, higher oligomeric, and monomeric states. From these two, only FS38 showed some dimeric species. Regarding SEC, only proteins FS58 and FS59 showed no aggregates and mainly dimeric species, as well as almost no higher oligomeric states. These two proteins seemed to be the only chimeras forming proper quaternary structures.

Overall, we observed a drastic decrease in expression yield. The introduction of artificial interfaces had serious effects on folding and formation of quaternary structures. Impurities may have contributed in our measurements. However, proteins were found to be quite stable. 
Fine-tuning might be needed in order to address these issues and improve protein-protein interactions. This requires a detailed understanding of both proteins. Thus, the KS-AT wild types were studied by mutagenesis.

A

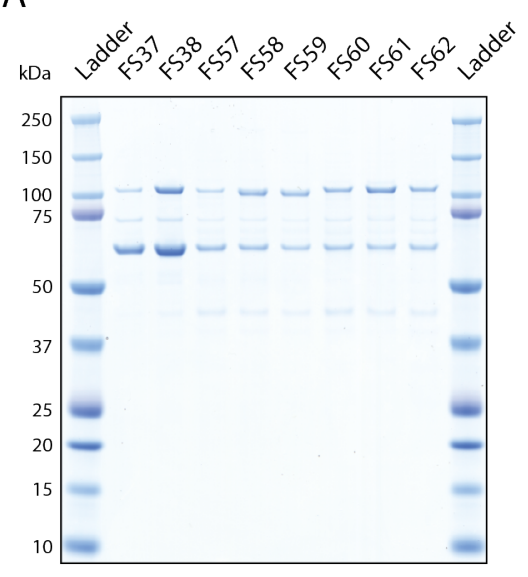

B

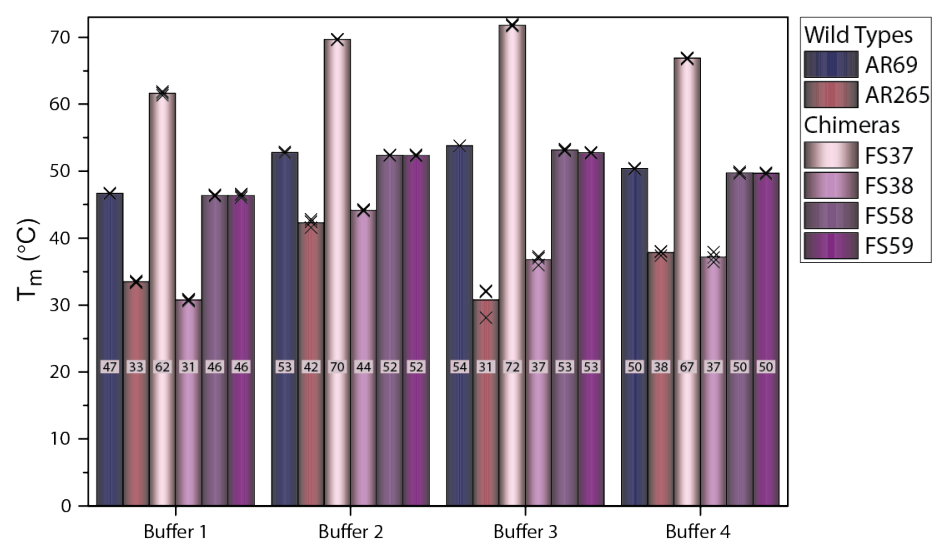

D

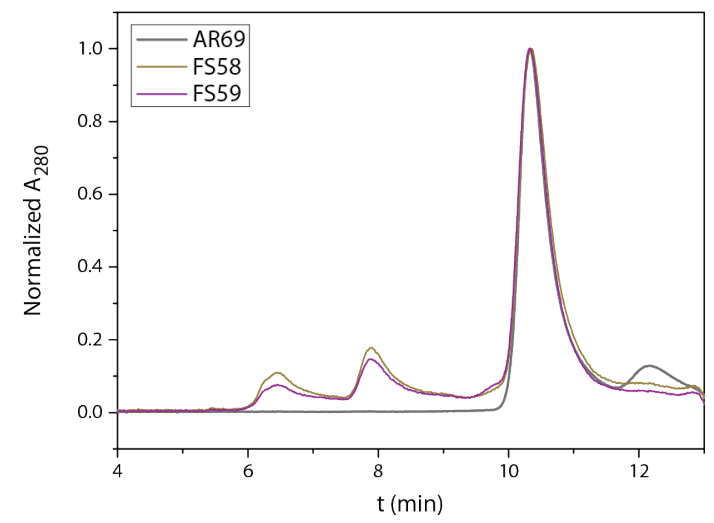

Figure 3.14: Analysis of KS-AT chimeras. (A) SDS-PAGE (NuPAGE Bis-Tris 4-12\%) of mFAS/DEBS3M5 chimeric KS-AT. Elution fractions after tandem affinity chromatography. (B) Average melting temperatures (in ${ }^{\circ} \mathrm{C}$ ) of mFAS and DEBS3M5 KS-AT wild types (AR69 and AR265) and of chimeric KS-AT constructs (FS37, FS38, FS58, FS59) in different buffers. Measured in triplicates. Crosses correspond to one biological replicate. Buffer 1: water, Buffer 2: Strep wash buffer pH 7.6, Buffer 3: Strep wash buffer $\mathrm{pH} 6.0$, Buffer 4: $40 \mathrm{mM}$ Tris, pH 7.6, $1 \mathrm{mM}$ EDTA, $10 \%$ glycerol. (C) and (D) Exemplary normalized size exclusion chromatogram of wild type AR69 and chimeric KS-AT constructs FS37, FS38, and FS57-FS62.

\subsubsection{Analysis of mFAS KS-AT Wild Type}

In order to identify hotspot residues which contribute to the formation of stable domain:domain interfaces in the KS-AT construct, single point mutations were introduced and their effects on protein's stability were analyzed. Expression yield, melting behavior, and oligomeric states served as readout. ${ }^{[17,20,225]}$ Different bioinformatics tools were used to predict interfacial hotspots using the mFAS KS-AT structure (chains A and/or D, PDB: 5MY0, see 


\section{Results}

section 5.1.14). Based on these predictions, the following residues were chosen as alanine mutation sites: D61 and N96 (residue numbering following UniProt entry P19096) located in the KS, interacting with the LD (Fig. 3.15). H425, R429, 1457, R468, Y470, V479, E481, and Q483 located in the LD and interacting with KS or AT. H804 and L805 in the AT domain, interacting with the LD. Predicted effects are summarized in Table 3.6. Dr. Karthik Paithankar performed the Rosetta calculations on the mFAS KS-AT wild type using chains A and D of the solved structure. [9]

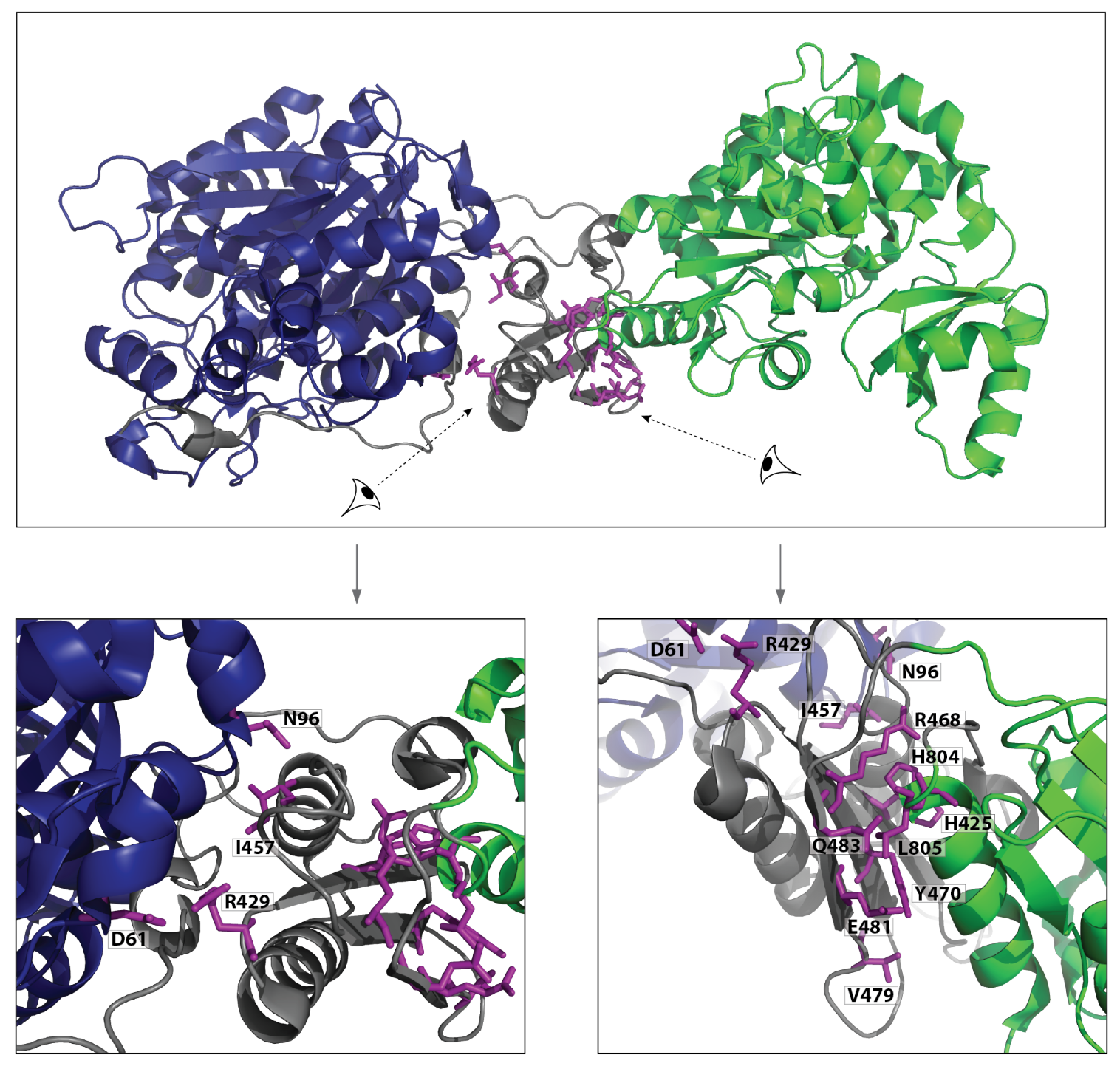

Figure 3.15: Sites for mutagenesis in mFAS KS-AT. Cartoon representation (PDB: 5MY0 ${ }^{[9]}$ ) with $\mathrm{KS}, \mathrm{LD}$, and AT depicted in blue, gray, and green, respectively. Interfacial residues between KS and LD as well as LD and AT chosen for mutagenesis are highlighted in purple. Residue numbering following UniProt entry P19096. 
Table 3.6: Results of bioinformatic analysis of mFAS KS-AT. Difference in the Gibbs free energy $(\Delta \Delta \mathrm{G})$ between mutant and wild type (in Rosetta energy unit (REU) and $\mathrm{kcal} / \mathrm{mol}$ ) predicted by different bioinformatics tools. For better comparison of results from different bioinformatics tools, values of mCSM are handled as described in section 5.1.14. SpotOn predicts mutations to be a Nullspot (not relevant for the interaction) or a Hotspot (relevant for the interaction). Residue numbering following UniProt entry P19096.

\begin{tabular}{|c|c|c|c|c|c|c|c|c|}
\hline \multirow{3}{*}{$\begin{array}{l}\text { Program } \\
\text { Chain } \\
\text { PAL }\end{array}$} & \multicolumn{4}{|c|}{ Rosetta } & \multirow{3}{*}{\multicolumn{2}{|c|}{\begin{tabular}{|c} 
Robetta \\
W/o \\
w/o
\end{tabular}}} & \multirow{3}{*}{$\begin{array}{c}\mathrm{mCSM} \\
\mathrm{w}\end{array}$} & \multirow{3}{*}{$\begin{array}{c}\text { SpotOn } \\
A \\
w / o\end{array}$} \\
\hline & \multicolumn{2}{|c|}{$A$} & \multicolumn{2}{|c|}{$\mathrm{D}$} & & & & \\
\hline & w & $w / o$ & w & $w / o$ & & & & \\
\hline Protein & \multicolumn{4}{|c|}{$\begin{array}{c}\Delta \Delta G \\
\text { (REU) }\end{array}$} & \multicolumn{3}{|c|}{$\begin{array}{c}\Delta \Delta \mathrm{G} \\
(\mathrm{kcal} / \mathrm{mol})\end{array}$} & $\begin{array}{l}\text { Hot/ } \\
\text { Null }\end{array}$ \\
\hline $\begin{array}{l}\text { FS39 } \\
\text { (D61A) }\end{array}$ & 7.34 & 4.59 & 6.63 & 3.84 & 1.52 & 1.98 & 1.05 & Null \\
\hline $\begin{array}{l}\text { FS40 } \\
(\mathrm{N} 96 \mathrm{~A})\end{array}$ & 5.71 & 5.71 & 6.20 & 6.20 & 3.26 & 2.11 & 0.181 & Null \\
\hline $\begin{array}{l}\text { FS41 } \\
\text { (R429A) }\end{array}$ & 3.34 & 3.64 & 3.60 & 3.92 & 3.02 & 1.04 & 0.263 & Null \\
\hline $\begin{array}{l}\text { FS42 } \\
(\text { I457A) }\end{array}$ & 4.86 & 3.10 & 5.19 & 3.44 & 1.61 & 2.17 & 0.424 & Hot \\
\hline $\begin{array}{l}\text { FS43 } \\
(\mathrm{R} 468 \mathrm{~A})\end{array}$ & 3.34 & 3.68 & 3.53 & 3.53 & 1.70 & 2.07 & 0.987 & Null \\
\hline $\begin{array}{l}\text { FS44 } \\
(Y 470 A)\end{array}$ & 2.11 & 2.11 & 3.51 & 3.51 & 2.75 & 4.28 & 0.537 & Hot \\
\hline $\begin{array}{l}\text { FS51 } \\
(\mathrm{H} 425 \mathrm{~A})\end{array}$ & 0.778 & 0.778 & 2.18 & 2.18 & 1.11 & 0.30 & 0.53 & Null \\
\hline $\begin{array}{l}\text { FS52 } \\
(\text { V479A) }\end{array}$ & 3.34 & 3.43 & 3.48 & 3.48 & 0.32 & 0.42 & 0.49 & Hot \\
\hline $\begin{array}{l}\text { FS53 } \\
(E 481 A)\end{array}$ & 3.17 & 3.17 & 4.16 & 4.16 & 0.59 & 0.09 & 0.68 & Hot \\
\hline $\begin{array}{l}\text { FS54 } \\
\text { (Q483A) }\end{array}$ & -4.62 & -4.62 & 3.22 & -2.28 & 0.96 & 0.18 & 0.159 & Hot \\
\hline $\begin{array}{l}\text { FS55 } \\
(\mathrm{H} 804 \mathrm{~A})\end{array}$ & 0.730 & 0.731 & 0.797 & 0.797 & 3.97 & 0.45 & 1.433 & Null \\
\hline $\begin{array}{l}\text { FS56 } \\
\text { (L805A) }\end{array}$ & -2.50 & -2.49 & 0.243 & -0.243 & 2.69 & 2.21 & 0.42 & Null \\
\hline
\end{tabular}


Maren Berlinghof cloned the mutants and isolated the wild type AR69 and mutants FS39FS44 as well as FS51-FS56 in biological triplicates during her master's thesis under my supervision. [223] Overall, introducing alanine mutations decreased expression yields (Tab. 3.7). Mutations at R468, Y470, and L805 (FS43, FS44, and FS56) showed only minor effects and gave wild type-like yields. Especially mutations introduced at D61 and 1457 (FS39 and FS42) seemed to strongly affect protein expressibility. All other proteins showed around $50 \%$ protein yield compared to the wild type. Overall, protein expression was clearly influenced, but yields of most proteins were still in a good range.

Table 3.7: Average expression yields of mFAS KS-AT wild type (AR69) and mutants (FS39FS44, FS51-FS56) after tandem affinity chromatography of $1 \mathrm{~L}$ expression culture. Yields determined from three independent expressions.

\begin{tabular}{lccc}
\hline Protein & Pellet's Weight $(\mathbf{g})$ & Total Yield $(\mathbf{m g})$ & Yield $(\mathbf{m g})$ per g Pellet \\
\hline AR69 & 9.17 & 15.7 & 1.71 \\
FS39 & 8.02 & 0.332 & 0.0414 \\
FS40 & 8.06 & 7.33 & 0.909 \\
FS41 & 7.73 & 6.05 & 0.783 \\
FS42 & 8.86 & 2.56 & 0.289 \\
FS43 & 9.11 & 12.2 & 1.34 \\
FS44 & 7.53 & 10.1 & 1.34 \\
FS51 & 8.23 & 7.74 & 0.940 \\
FS52 & 7.97 & 7.89 & 0.990 \\
FS53 & 7.44 & 8.51 & 1.14 \\
FS54 & 8.03 & 8.32 & 1.04 \\
FS55 & 7.25 & 8.46 & 1.17 \\
FS56 & 7.43 & 9.83 & 1.32 \\
\hline
\end{tabular}

SDS-PAGE analysis showed a single distinct protein band for full-length protein (Fig. 3.16). FS39 (D61A) showed an intense additional band of around $60 \mathrm{kDa}$, which was also found for FS42 (I457A) to a much lesser extent. Mutation of D61 (and 1457 to a lesser extent) seemed to destabilize the protein, leading to protein degradation, as observed in SDS-PAGE. A band of the same size was also observed in SDS-PAGE of chimeric KS-AT and chimeric full-length mFAS constructs and could correspond to KS-LD. The other mFAS KS-AT mutants showed wild type-like behavior in SDS-PAGE. This indicates that amino acids D61 and 1457 might be crucial for the KS-AT fold, whereas mutation of the other residues does not show drastic effects on protein stability. Interestingly, the same protein degradation was observed in chimeras when swapping solely the AT domain, preserving the natural KS-LD interaction. 


\section{Results}

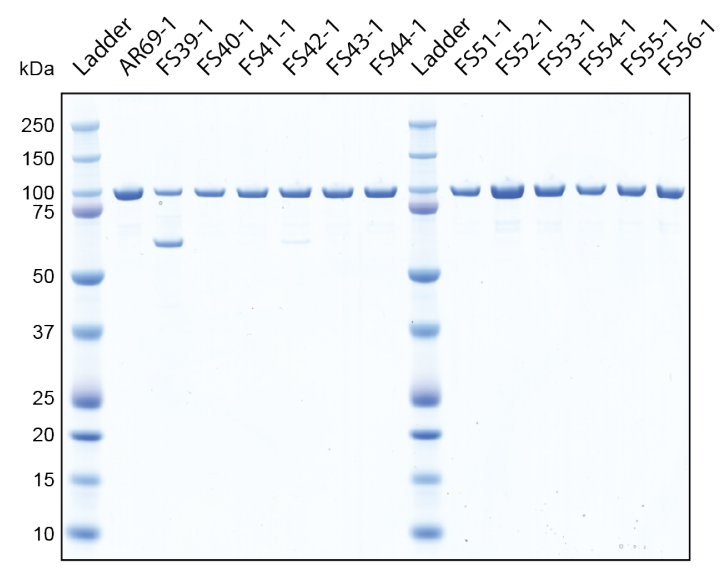

Figure 3.16: Exemplary SDS-PAGE analysis (NuPAGE Bis-Tris 4-12\%) of one biological replicate of mFAS KS-AT wild type (AR69) and mutants (FS39-FS44, FS51-FS56) of concentrated elution fractions after tandem affinity chromatography.
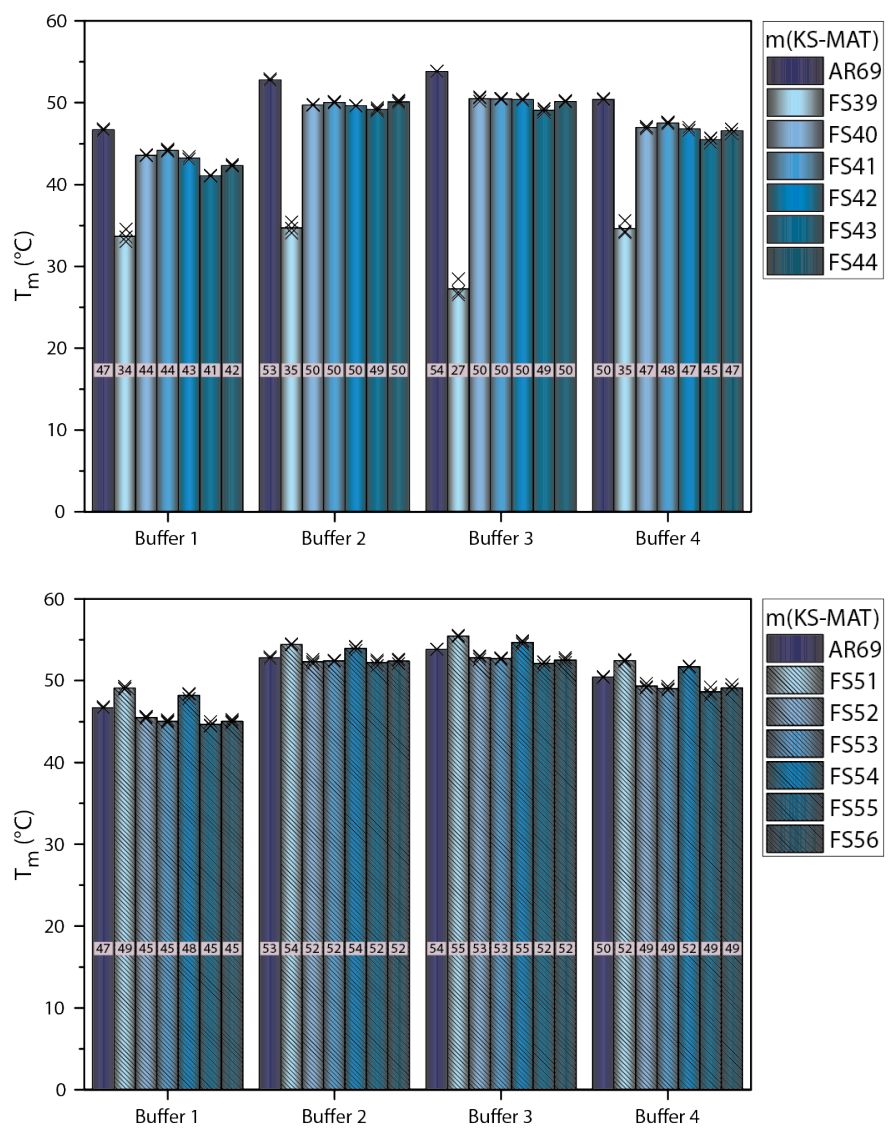

Figure 3.17: Average melting temperatures (in ${ }^{\circ} \mathrm{C}$ ) of mFAS KS-AT wild type (AR69) and mutants (FS39-FS44, FS51-FS56) in different buffers. Measured in triplicates. Crosses correspond to one biological replicate. Buffer 1: water, Buffer 2: Strep wash buffer pH 7.6, Buffer 3: Strep wash buffer pH 6.0, Buffer 4: $40 \mathrm{mM}$ Tris, pH 7.6, 1 mM EDTA, $10 \%$ glycerol. 
TSA further fortified the role of site D61. While all other mutants showed wild type-like melting behavior in all buffers tested, the melting temperatures were significantly decreased for mutant FS39 (D61A), indicating drastically reduced protein stability (Fig. 3.17). This different melting behavior could be (in parts) caused by degraded protein in the sample. However, in case of the KS-AT chimeras, protein degradation did not lower the melting temperature at all or at least not to this extent. Thus, the stability of the protein seemed to be significantly lowered by this mutation (D61A). Notably, FS42 (1457A) showed wild type-like behavior in the TSA.

Finally, SEC confirmed that all tested amino acids are quite robust to mutation, except for D61 (Fig. 3.18): Only mutant FS39 (D61A) showed different oligomeric states than the wild type, forming mainly higher oligomeric states and monomers, almost no dimeric species was found. In contrast, all other eleven proteins formed mainly the dimeric, partly the monomeric state, just like the wild type AR69. Notably, also FS42 (I457A) shows wild-type like behavior. Apparently the protein is overall quite stable and well folded. Like the SDS-PAGE showed, protein degradation occured only to a minor extent.

A

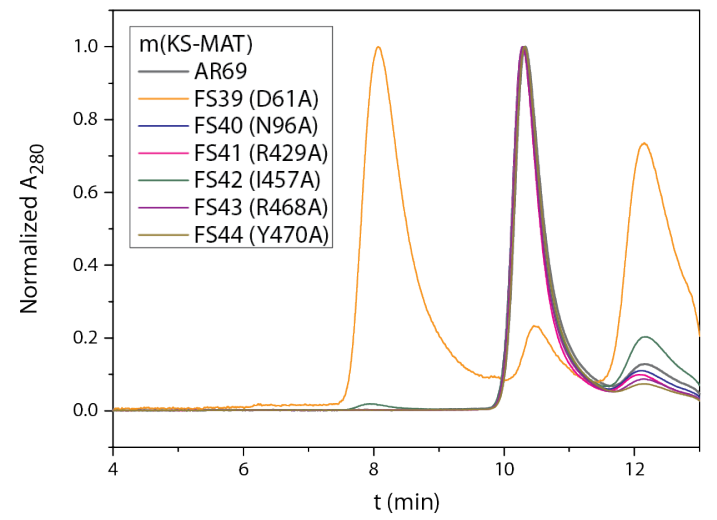

B

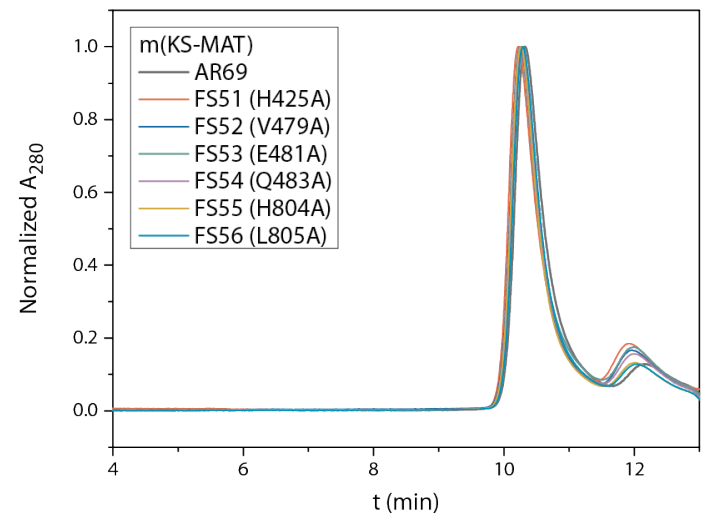

Figure 3.18: Exemplary normalized size exclusion chromatogram of mFAS KS-AT wild type (AR69) and mutants FS39-FS44 (A) and mutants FS51-FS56 (B).

All experiments showed that residue D61 is important for correct folding of mFAS KS-AT. This residue was selected for mutagenesis due to its involvement in the salt bridge between KS and LD domain (Fig. 3.19). Interestingly, mutation of its counterpart R429 to alanine (FS41) did not have the same destabilizing effect. 


\section{Results}

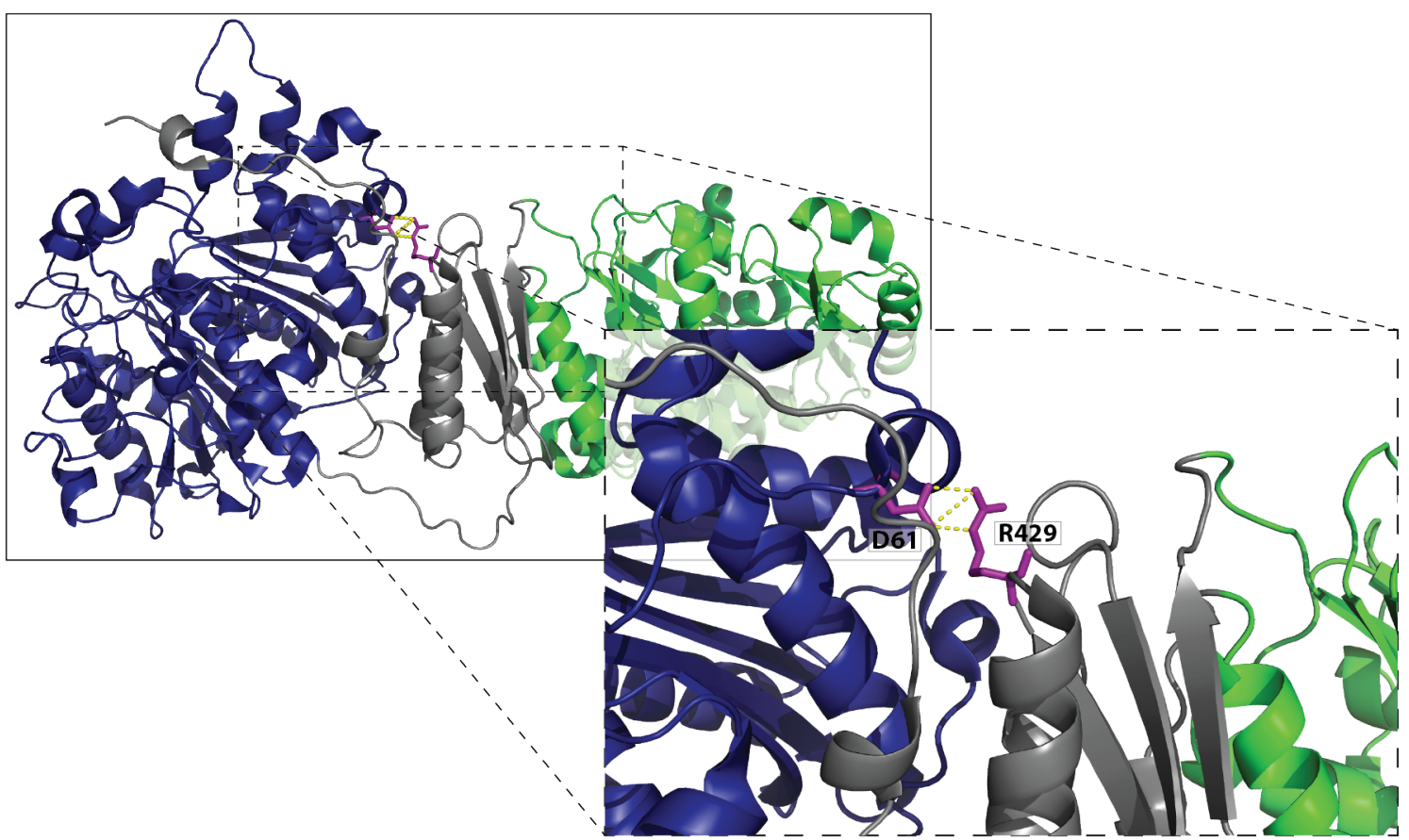

Figure 3.19: Cartoon representation of the interfacial salt bridge formed between D61 and R429 in mFAS KS-AT (PDB: 5MY0 ${ }^{[9]}$ ). KS, LD, and AT shown in blue, gray, and green, respectively. Residues D61 and R429 depicted as purple sticks. Salt bridge depicted in yellow and calculated using PyMOL. Residue numbering following UniProt entry P19096.

Overall, the mFAS KS-AT construct seemed to be quite robust to mutations in its interfaces and therefore is a promising candidate for AT swapping approaches. Only mutation D61A destabilized the didomain severely, which was reflected in drastically reduced yield and melting temperatures as well as changes in oligomeric states. Additionally, mutant 1457A showed some destabilizing effects, manifested in significantly reduced yields and minor protein degradation, but showed wild type-like behavior in TSA and SEC. Although some other mutants decreased the expression yield, proteins still seemed to be folded correctly and behaved like the wild type in TSA and SEC. Even mutation of R429, D61's counterpart in the salt bridge stabilizing the KS-LD interaction, led to stable protein. These results demonstrate the high robustness of mFAS KS-AT and prove that AT and LD-AT swapping should be possible within the mFAS. 


\section{Results}

\subsubsection{Analysis of DEBS3M5 KS-AT Wild Type}

Six different point mutations were chosen within DEBS3M5 KS-AT (Fig. 3.20). Effects of muations predicted by the different bioinformatics tools using the DEBS3M5 KS-AT structure (chains A and/or D, PDB: 2HG4) are summarized in Table 3.8. Dr. Karthik Paithankar performed the Rosetta calculations on the DEBS3M5 KS-AT wild type using chains A and D of the solved structure. [99]

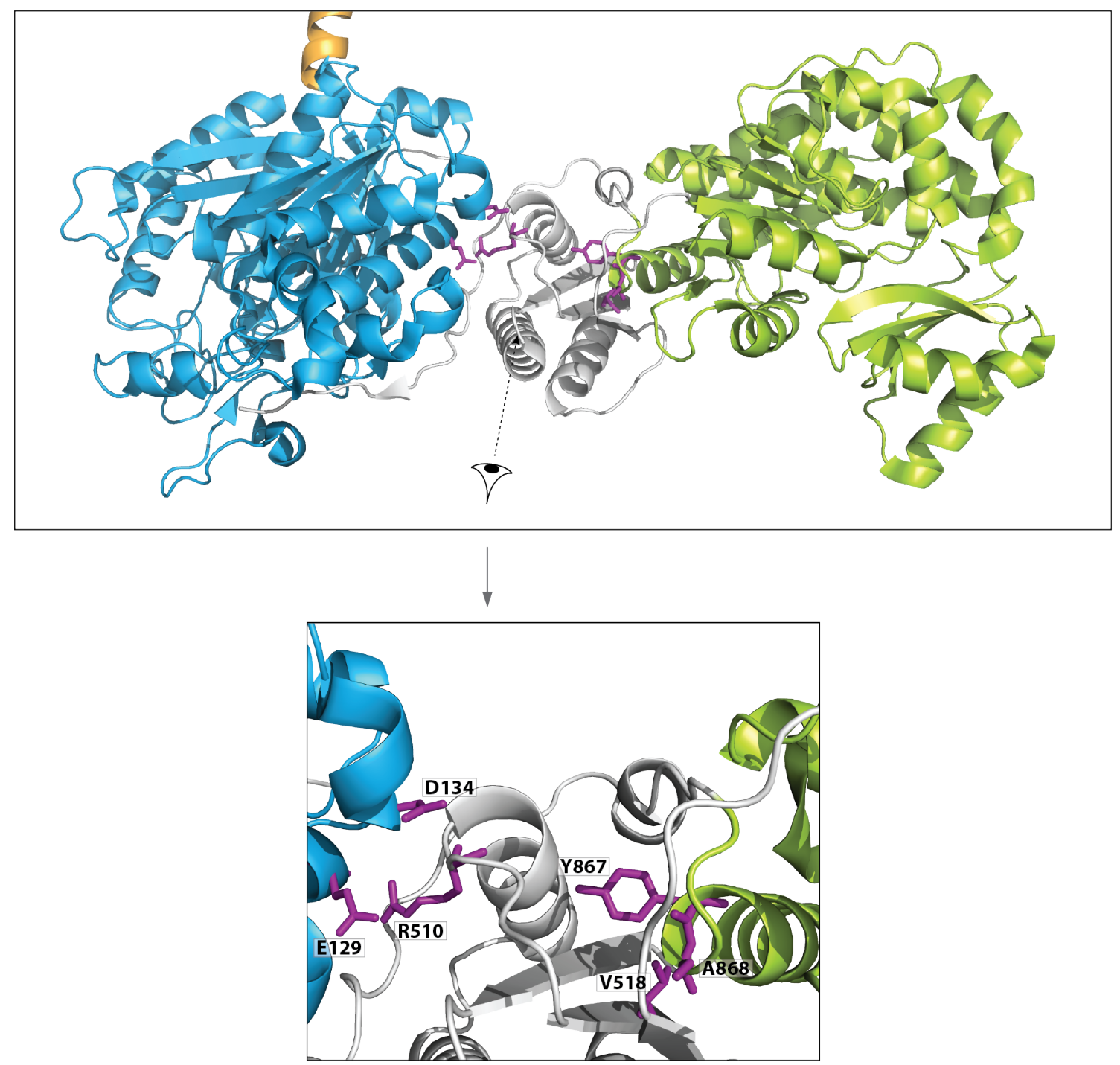

Figure 3.20: Sites for mutagenesis in DEBS3M5 KS-AT wild type. Cartoon representation (PDB: $2 \mathrm{HG}_{4}{ }^{[99]}$ ) with $\mathrm{KS}, \mathrm{LD}$, and AT depicted in light blue, light gray, and lemon, respectively. Interfacial residues between KS and LD as well as LD and AT chosen for mutagenesis are highlighted in purple. Residue numbering following UniProt entry Q03133. 
Table 3.8: Results of bioinformatic analysis of DEBS3M5 KS-AT. Difference in the Gibbs free energy $(\Delta \Delta \mathrm{G})$ between mutant and wild type (in Rosetta energy unit (REU) and $\mathrm{kcal} / \mathrm{mol}$ ) predicted by different bioinformatics tools. For better comparison of results from different bioinformatics tools, values of $\mathrm{mCSM}$ are handled as described in section 5.1.14. Mutations are predicted to be a Nullspot (not relevant for the interaction) or a Hotspot (relevant for the interaction) via SpotOn. Since Robetta and DrugScore are limited to alanine mutations, no prediction could be made for FS50 (A868R). n. a.: not available. Residue numbering following UniProt entry Q03133.

\begin{tabular}{|c|c|c|c|c|c|c|c|c|}
\hline \multirow{3}{*}{$\begin{array}{l}\text { Program } \\
\text { Chain } \\
\text { PAL }\end{array}$} & \multicolumn{4}{|c|}{ Rosetta } & \multirow{3}{*}{\multicolumn{2}{|c|}{$\begin{array}{r}\text { Robetta } \\
\mathrm{w} / \mathrm{o} \\
\end{array}$}} & \multirow{3}{*}{$\begin{array}{c}\mathbf{m C S M} \\
\mathrm{w}\end{array}$} & \multirow{3}{*}{$\begin{array}{c}\text { SpotOn } \\
A \\
w / o\end{array}$} \\
\hline & \multicolumn{2}{|c|}{ A } & \multicolumn{2}{|c|}{$\mathrm{D}$} & & & & \\
\hline & w & $w / o$ & w & $w / o$ & & & & \\
\hline Protein & \multicolumn{4}{|c|}{$\begin{array}{c}\Delta \Delta G \\
(R E U)\end{array}$} & \multicolumn{3}{|c|}{$\begin{array}{c}\Delta \Delta \mathrm{G} \\
(\mathrm{kcal} / \mathrm{mol})\end{array}$} & $\begin{array}{l}\text { Hot/ } \\
\text { Null }\end{array}$ \\
\hline $\begin{array}{l}\text { FS45 } \\
(\text { E129A) }\end{array}$ & 4.27 & 0.856 & 4.70 & 1.26 & 0.48 & -0.18 & 1.42 & Null \\
\hline $\begin{array}{l}\text { FS46 } \\
\text { (D134A) }\end{array}$ & 0.550 & 0.592 & 3.96 & 3.96 & -0.50 & 3.73 & 3.23 & Null \\
\hline $\begin{array}{l}\text { FS47 } \\
\text { (R510A) }\end{array}$ & 2.56 & 2.42 & 2.46 & 1.08 & 0.40 & 0.54 & 1.57 & Null \\
\hline $\begin{array}{l}\text { FS48 } \\
(\text { V518A) }\end{array}$ & 6.65 & 5.64 & 6.59 & 5.64 & 0.90 & 0.84 & 1.00 & Hot \\
\hline $\begin{array}{l}\text { FS49 } \\
(\text { Y867A) }\end{array}$ & 4.51 & 3.90 & 2.18 & 1.76 & 1.90 & 3.29 & 1.31 & Null \\
\hline $\begin{array}{l}\text { FS50 } \\
(\mathrm{A} 868 \mathrm{R})\end{array}$ & 100.5 & 78.4 & 17.3 & 18.0 & n. a. & n. a. & 3.24 & Null \\
\hline
\end{tabular}

Maren Berlinghof cloned the mutants and isolated the wild type AR265 and mutants FS45-

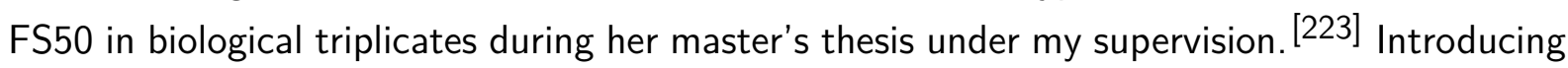
any point mutations decreased expression yields drastically for all sites (Tab. 3.9). While differences between mutant and wild type yields were very pronounced, all mutant yields were very low and did not show significant differences between each other. 
Table 3.9: Average expression yields of DEBS3M5 KS-AT wild type (AR265) and mutants (FS45-FS50) after tandem affinity chromatography of $1 \mathrm{~L}$ expression culture. Yields determined from three independent expressions.

\begin{tabular}{lccc}
\hline Protein & Pellet's Weight $(\mathbf{g})$ & Total Yield $(\mathbf{m g})$ & Yield $(\mathbf{m g})$ per g Pellet \\
\hline AR265 & 8.83 & 11.3 & 1.28 \\
FS45 & 8.67 & 0.0269 & 0.00310 \\
FS46 & 7.48 & 0.0224 & 0.00299 \\
FS47 & 7.73 & 0.0192 & 0.00248 \\
FS48 & 6.63 & 0.0637 & 0.00961 \\
FS49 & 9.92 & 0.0985 & 0.00993 \\
FS50 & 8.00 & 0.101 & 0.0126 \\
\hline
\end{tabular}

Despite the low expression yield, mutants FS45-FS48 were pure after tandem affinity chromatography (Fig. 3.21). Elution fractions of mutants FS49 and FS50 showed a band shift and also impurities of higher molecular weight proteins. These have already been observed in the cell lysate and most likely were E. coli proteins. Depending on protein handling, some replicates showed these impurities, others did not. Almost no protein degradation was observed. Due to the very low yields and impurities, further analysis of the proteins was difficult and gave no reliable results.

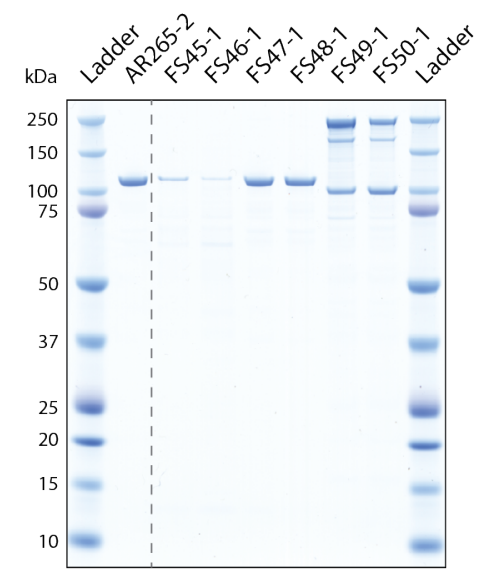

Figure 3.21: Analysis of DEBS3M5 KS-AT wild type (AR265) and mutants (FS45, FS48-FS50). Exemplary SDS-PAGE (NuPAGE Bis-Tris 4-12\%) of one biological replicate. Concentrated elution fractions after tandem affinity chromatography.

Since the expression yield is a measure for stability, ${ }^{[20,225]}$ the drastically reduced mutant yields compared to the wild type indicate that single point mutations introduced at interfaces within the DEBS3M5 KS-AT didomain led to severe protein destabilization. In contrast to the mFAS, DEBS3M5 was not robust at all to mutagenesis and seemed to be unsuitable for AT 
domain swapping approaches. This might be the reason why the design of mFAS/DEBS3M5 chimeras was not successful. In the following, our results were used to correlate the outcome of experiments to the prediction of the different bioinformatics tools.

\subsubsection{Correlation of Experimental and in silico Data}

We compared the experimental data on mFAS and DEBS3M5 KS-AT wild type with the prediction of the different bioinformatics tools to see which of these tools are helpful or whether they are useful at all in engineering approaches of complex interfaces and to see which of our analysis methods can serve as reliable readout.

This section will solely focus on the bioinformatics tool Rosetta. Within the set of tools used in this study, it is the one which uses the most complex algorithm to predict effects of mutations on the stability of proteins. I expected the predictions of this elaborate tool to be more reliable than the simpler web-based programs. Correlation of the experimental output yields, melting temperatures, and oligomeric states confirmed this expectation: Overall, only predictions of Rosetta showed correlations to experimental output, which will be shown in the following. Predictions of the other bioinformatics tools showed no or only partly correlation tendencies. This is exemplarily shown in Figure S17. Some bioinformatics tools (Robetta, DrugScore, SpotOn) could only be used without the PAL. Thus, we performed Rosetta calculations additionally without PAL to see whether this impairs the correlations. Furthermore, we wanted to analyze effects of different chains on the prediction. All bioinformatics tools were used for analysis of chain A. We performed analysis with Rosetta additionally for chain $D$, which shows the largest and second largest RMSD to chain A in DEBS3M5 KS-AT (PDB: $2 \mathrm{HG} 4,{ }^{[99]} 0.852 \AA$ ) and in mFAS KS-AT (PDB: 5MY0, ${ }^{[9]} 0.480 \AA ̊$ ), respectively.

\section{Correlation of Experimental and in silico Data for mFAS KS-AT Wild Type}

Overall, the predictions for chains A and D gave different values for the change in Gibbs free energy (Tab. 3.6). However, the discrepancy between different mutations is similar and, thus, similar correlation tendencies should occur for chains A and D. Regarding predictions with and without PAL for chain $A$ and chain $D$, most mutants remained unaffected. Solely mutants D61A and 1457A showed a significant shift in predicted energy change: Without PAL, these mutants were predicted to effect stability to a lesser extent than with $P A L$, indicating that sites D61 and 1457 interact with this linker. This should clearly influence correlation tendencies.

Expression yields served as first readout for effects on protein stability. ${ }^{[20,225]}$ To see, whether there is a correlation, the expression yield was plotted against the predicted change in Gibbs 
free energy (Fig. 3.22A). Positive changes correspond to destabilizing effects, while negative $\Delta \Delta \mathrm{G}$ values indicate stabilizing effects. Mutations with higher change in the Gibbs free energy are expected to influence the stability to a higher degree. Most proteins showed wild typelike expression levels, which can be explained by small changes in the Gibbs free energy of \pm 3.5 Rosetta energy units (REU). For higher energy changes, there was a tendency towards lower expression yields. Overall, predictions without PAL showed a similar correlation trend (Fig. 3.22B), although the predicted values differed slightly. However, the predictions of the two mutants with the lowest yields (D61A and 1457A) were significantly shifted towards lower energy changes, impairing the correlation. This can be explained by interactions of the sites D61 and 1457 with the PAL. Experimental and in silico analysis showed their importance in the didomain. Mutation of D61 led to severe destabilization, mutation of 1457 showed partly destabilization. Chain D with and without PAL (Fig. 3.22C and D) showed similar predictions and, hence, similar correlation tendencies as chain $\mathrm{A}$.

A

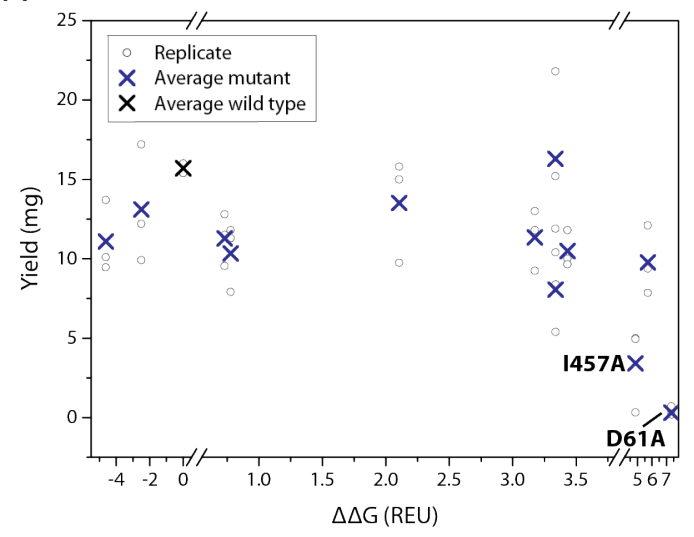

C

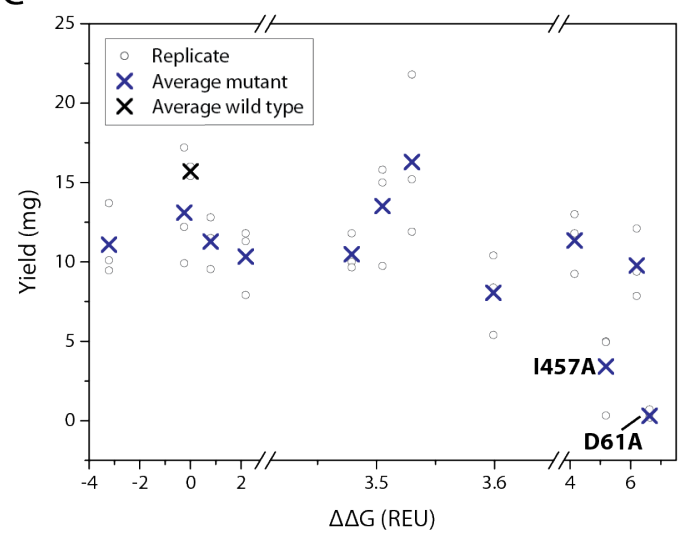

B

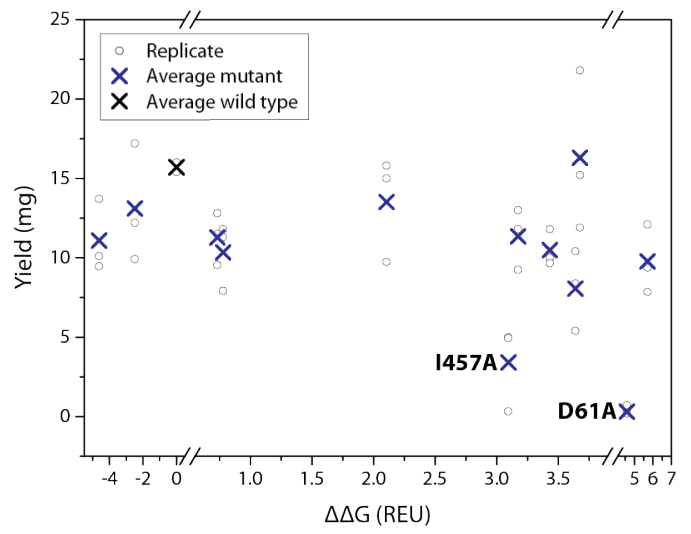

D

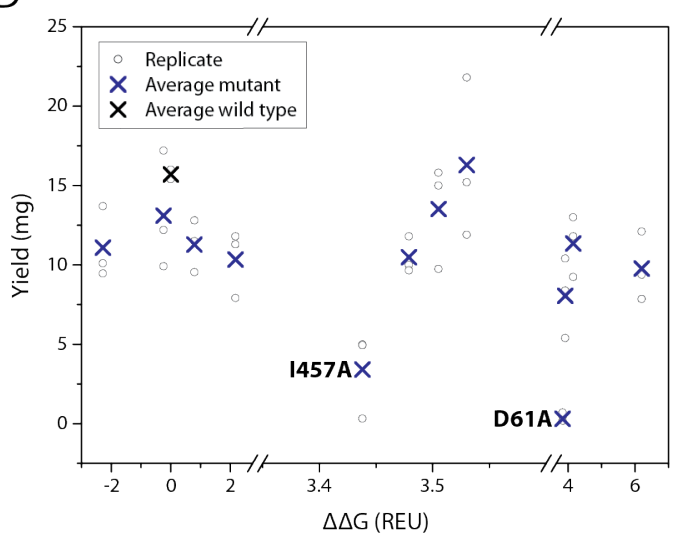

Figure 3.22: Yields (in $\mathrm{mg}$ per $1 \mathrm{~L}$ expression culture) of mFAS KS-AT wild type and mutants correlated to changes in the Gibbs free energy ( $\triangle \Delta G$ in REU) predicted by Rosetta. (A) Chain A with PAL. (B) Chain A without PAL. (C) Chain D with PAL. (D) Chain D without PAL. Black circles indicate the yields of biological replicates. Average yields of biological triplicates of wild type and mutants are indicated by black and blue crosses, respectively. Residues D61 and 1457 are labeled for clarity. Please mind the scales of the different $\mathrm{x}$-axis sectors. 
Melting temperatures served as second experimental readout. Differences in melting temperatures $\left(\Delta T_{m}=T_{m}\right.$ (Wild Type)- $T_{m}$ (Mutant) ) were plotted against changes in the Gibbs free energy (Fig. 3.23A). Negative energy changes have stabilizing effects and are expected to increase melting temperatures. $\Delta T_{m}$ should be positive and corresponding mutants should be located in the second quadrant. Positive energy changes should be destabilizing and should lower melting temperatures leading to a negative $\Delta T_{m}$. Mutants should be located in the fourth quadrant. Most mutants behaved as expected and there is a clear correlation between the difference in melting temperature and the predicted energy change. Overall, predictions without PAL (Fig. 3.23B) with shifted predictions for mutants D61A and I457A impaired this correlation due to their interaction with PAL. Correlations with predictions for chain $\mathrm{D}$ with and without PAL are shown in Figure 3.23C and D.

A

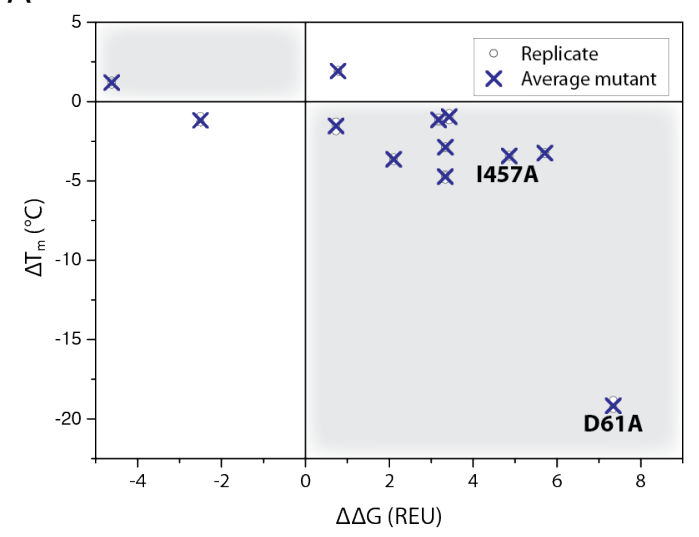

C

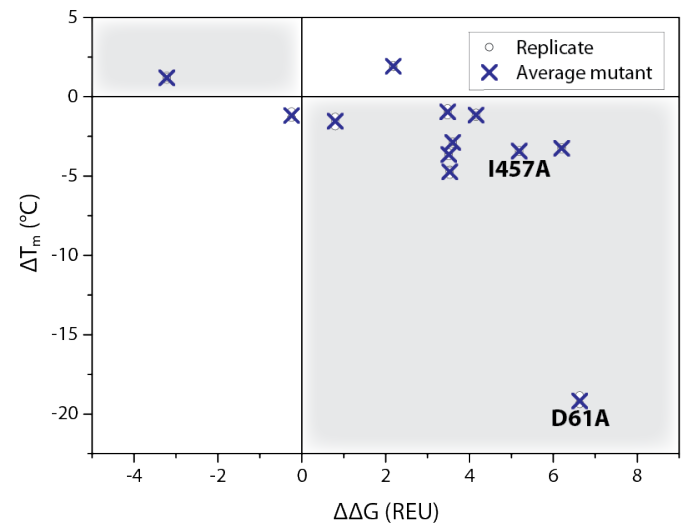

B

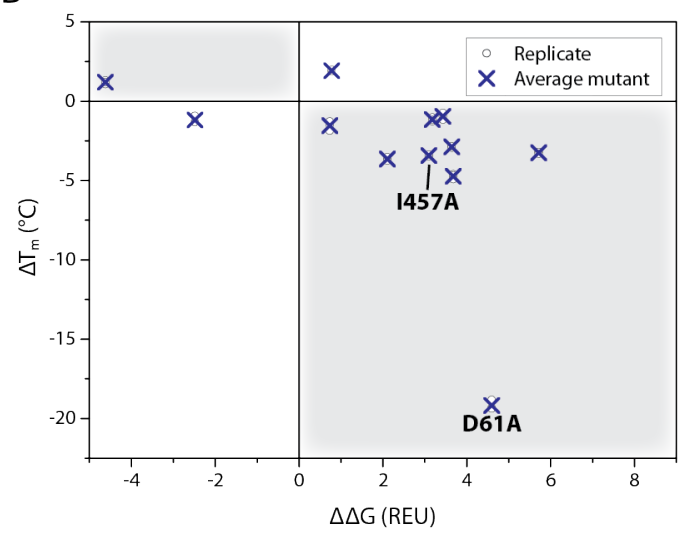

D

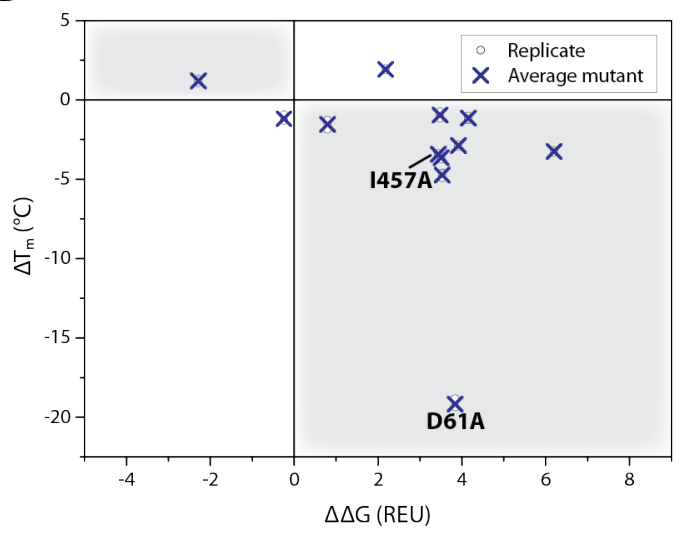

Figure 3.23: Changes in melting temperatures $\left(\Delta T_{m}\right.$ in $\left.{ }^{\circ} \mathrm{C}\right)$ of mFAS KS-AT mutants correlated to changes in the Gibbs free energy $(\triangle \Delta G$ in REU) predicted by Rosetta. (A) Chain A with PAL. (B) Chain A without PAL. (C) Chain D with PAL. (D) Chain D without PAL. Gray areas indicate quadrants, which would show a correlation between experimental and predicted output. Black circles indicate the melting temperatures of biological replicates. Average of biological triplicates is indicated by blue crosses. Residues D61 and 1457 are labeled for clarity. 
Oligomeric states served as third readout. The mFAS KS-AT wild type showed mainly dimeric and partly monomeric species (Fig. 3.18). Most of the mutants showed a similar behavior. In order to evaluate correlation, the ratio of dimer to monomer was plotted against changes in the Gibbs free energy (Fig. 3.24A). Only mutant D61A showed mainly higher oligomeric species. These results were not correlated, but the average ratio of higher oligomeric states to the sum of dimeric and monomeric species was 0.9 . This indicates strong differences compared to the wild type. Overall, the ratio of dimeric to monomeric species of most mutants is very similar to the wild type and is even increased. Similar to the correlation of expression yields, this ratio dropped for high positive energy changes and a correlation tendency emerged. This trend was impaired for correlation without the PAL (Fig. 3.24B) due to D61's and 1457's interactions with the linker. Correlations with predictions for chain D with and without PAL are shown in Figure 3.24C and D.

A

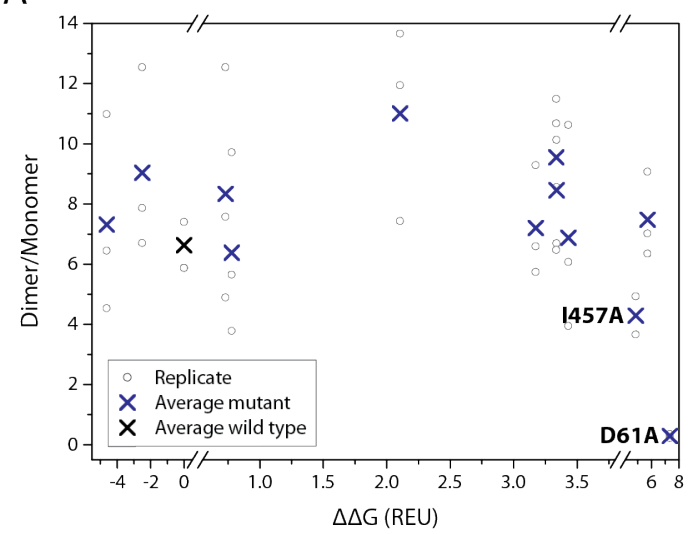

C

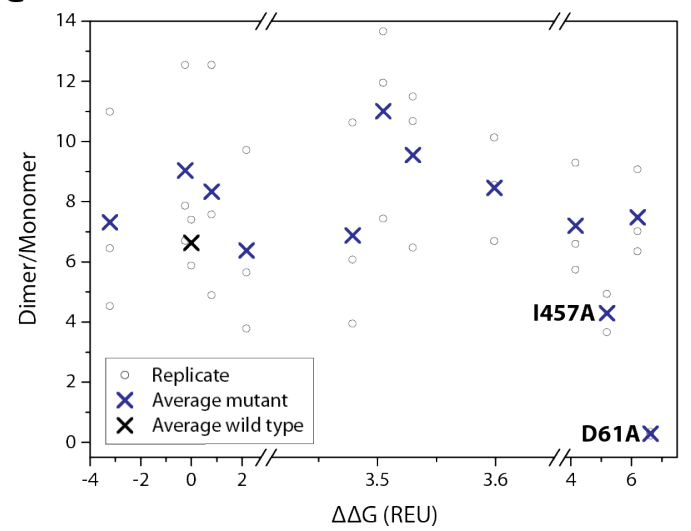

B

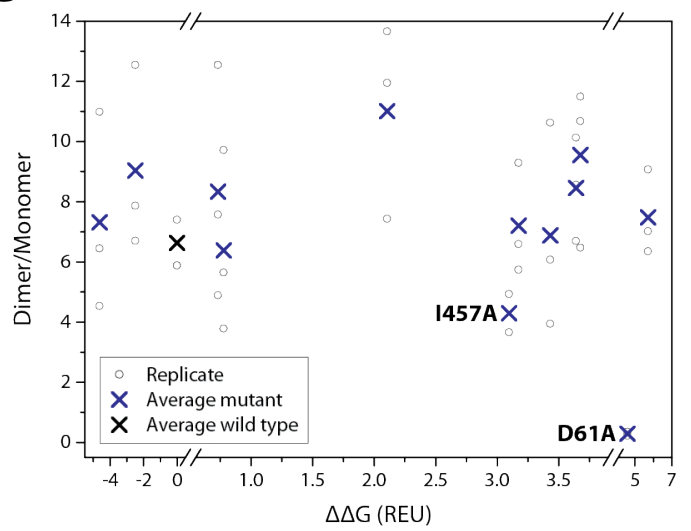

D

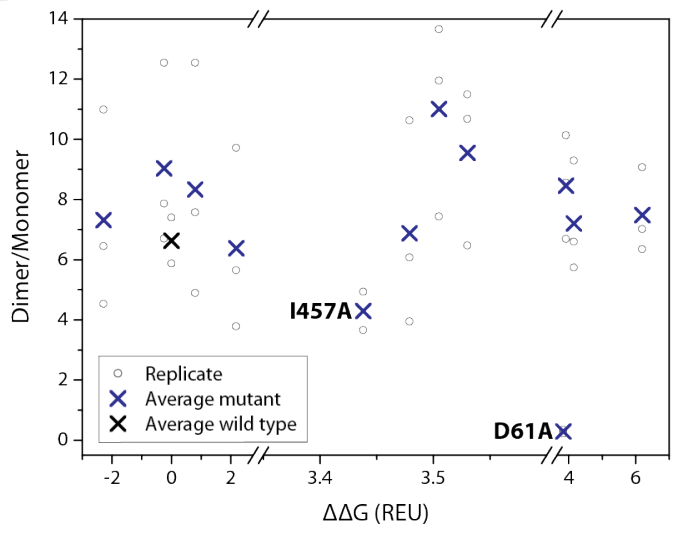

Figure 3.24: Ratio of monomeric to dimeric species of mFAS KS-AT wild type and mutants correlated to changes in the Gibbs free energy ( $\triangle \Delta G$ in REU) predicted by Rosetta. (A) Chain $A$ with PAL. (B) Chain A without PAL. (C) Chain D with PAL. (D) Chain D without PAL. Positive and negative $\Delta \Delta G$ correspond to destabilizing and stabilizing effects, respectively. Black circles indicate the yields of biological replicates. Average yields of biological triplicates of wild type and mutants are indicated by black and blue crosses, respectively. Residues D61 and 1457 are labeled for clarity. Please mind the scales of the different $\mathrm{x}$-axis sectors. 


\section{Results}

Overall, experimental results were found to correlate with the predicted effects irrespectively of which chain was used for the prediction. As expected, predictions without PAL influenced solely residues interacting with the linker. Since the number of those residues is limited, correlation tendencies were still observed. Both experimental and in silico data showed that residues D61 and 1457 are important for protein stability. Each experimental output used for estimation of protein stability was overall suited. All three methods clearly identified mutant D61A as highly destabilizing.

\section{Correlation of Experimental and in silico Data for DEBS3M5 KS-AT Wild Type}

For the DEBS3M5 KS-AT, only six mutants were created, leading to less data points for correlation. Overall, predictions in energy change for chain $A$ and $D$ are quite similar (Tab. 3.8). Mutants D134A and Y867A gave slightly different values. Highly remarkable are the predicted strong effect of mutant A868R as well as the high discrepancy between the predicted values for the two chains. Overall, chains $A$ and $D$ are expected to show different correlation tendencies. Calculations without the PAL lowered only the predicted effect of mutant E129A, which indicates that the site E129 is interacting with this linker. 


\section{Results}

All mutations introduced led to a severe drop in expression yields. No correlation between the predicted changes in the Gibbs free energy was observed (Fig. 3.25): Mutants predicted to have higher destabilizing effects showed rather higher yields than mutants with lower predicted effects. This might be caused by the drastically low yields, which make a precise determination of these effects impossible. Mutations introduced at the interfaces might destabilize the protein to an extent that the experimental readout "expression yield" is insufficiently sensitive towards the subtle differences between mutants and delivers no distinguishable results.

A

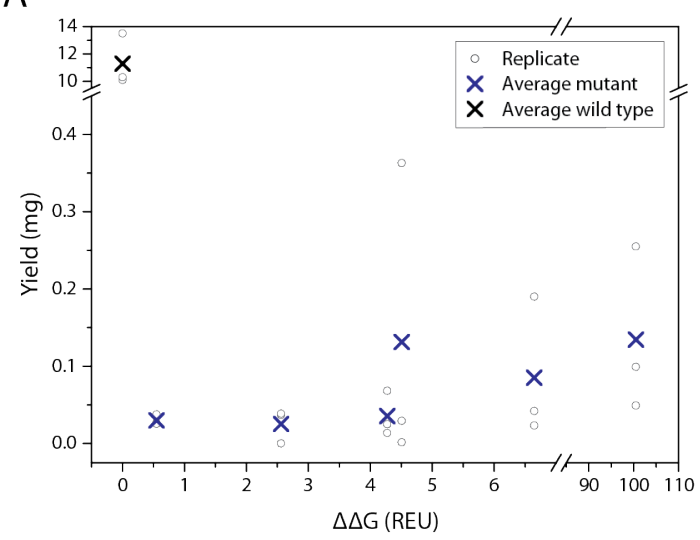

C

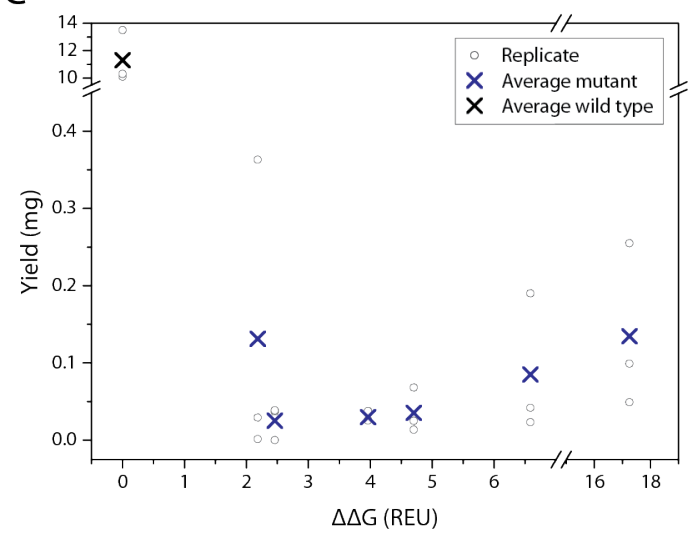

B

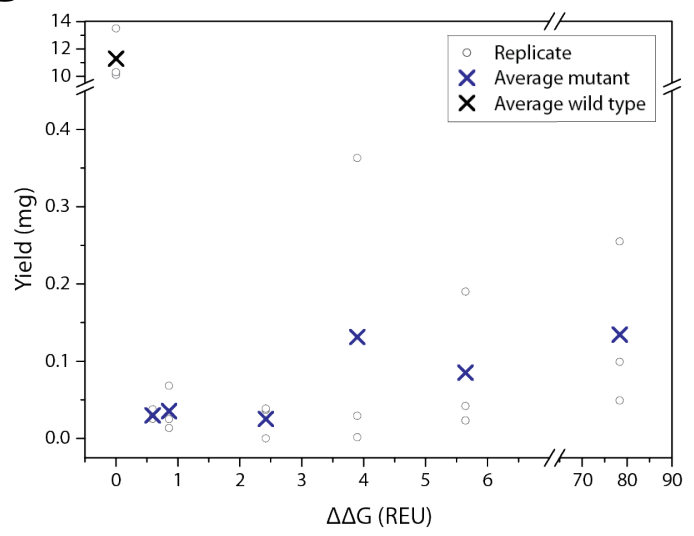

D

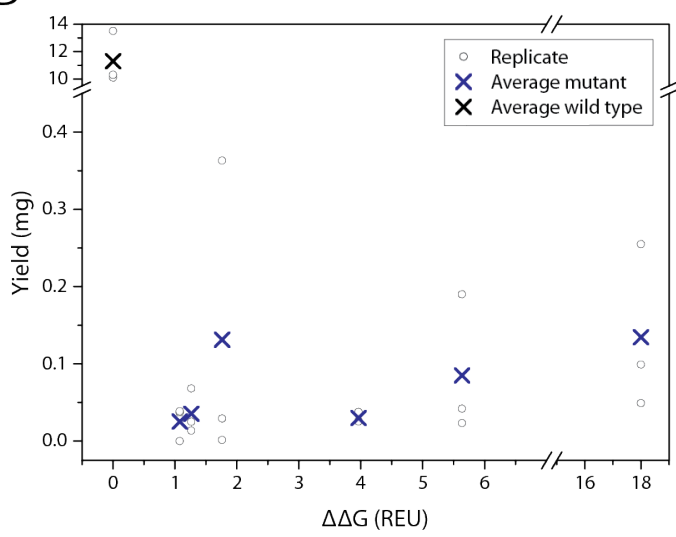

Figure 3.25: Yields (in mg per $1 \mathrm{~L}$ expression culture) of DEBS3M5 KS-AT wild type and mutants correlated to changes in the Gibbs free energy ( $\Delta \Delta G$ in REU) predicted by Rosetta. (A) Chain A with PAL. (B) Chain A without PAL. (C) Chain D with PAL. (D) Chain D without PAL. Positive and negative $\Delta \Delta G$ correspond to destabilizing and stabilizing effects, respectively. Black circles indicate the yields of biological replicates. Average yields of biological triplicates of wild type and mutants are indicated by black and blue crosses, respectively. Please mind the scales of the different $x$ - and $y$-axis sectors. 


\subsubsection{Generation of mFAS/FabD KS-AT Chimeras}

Since the AT domain from DEBS3M5 was not suited for domain swapping, we designed new chimeras with the AT (FabD) from E. coli FAS, which shows a specificity for elongation substrates (Mal, MMal) over the priming substrate Ac (section 3.3.6 and reference ${ }^{[216]}$ ). FabD and mMAT are overall similar: They share a sequence identity of $23 \%$ and their structural alignment gave an RMSD of $2.823 \AA$. In conformity with the DEBS3M5 chimera design, we created two chimeras with slightly different domain boundaries (Fig. 3.26).

$\begin{array}{ll}\text { AR69 } & \text { mFAS(KS-LD-AT) } \\ \text { FS116 } & \text { mFAS(KS-LD)-FabD } \\ \text { FS117 } & \text { mFAS(KS-LD)-FabD } \\ \text { AR90 } & \text { FabD }\end{array}$

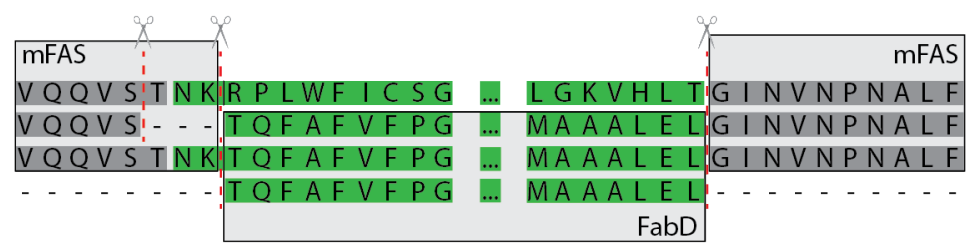

Figure 3.26: Domain boundaries for AT swapping with FabD. Two alternative boundaries were chosen for the first interface (mFAS(KAL):FabD), leading to the two chimeras FS116 and FS117.

Table 3.10: Test expression yields of KS-AT chimeras after tandem affinity chromatography of $1 \mathrm{~L}$ expression culture.

\begin{tabular}{lccc}
\hline Protein & Pellet's Weight $(\mathrm{g})$ & Total Yield $(\boldsymbol{\mu g})$ & Yield $(\boldsymbol{\mu g})$ per g Pellet \\
\hline FS116 & 21.5 & 828 & 38.5 \\
FS117 & 24.5 & 973 & 39.7 \\
\hline
\end{tabular}

Test expression of both proteins resulted in comparable yields (Tab. 3.10). SDS-PAGE analysis of the concentrated elution fractions showed mainly full-length protein (FS116: $94 \mathrm{kDa}$, FS117: $95 \mathrm{kDa}$ ), but also degraded proteins (Fig. 3.27A). Proteins were further analyzed by TSA. Both chimeras showed high start fluorescences, which indicates unfolded protein. ${ }^{\text {[224] }}$ Melting temperatures were significantly decreased compared to the wild type mFAS KS-AT and E. coli FabD (Fig. 3.27B). HPLC analysis showed no defined dimeric species and rather higher oligomeric states (Fig. 3.27C). Since no mFAS KS-AT wild type was available, the DEBS3M5 KS-AT served as reference. All these results pointed out poor stability of the mFAS/FabD chimeras. Nevertheless, FabD's activity in the chimeric didomain was analyzed using the established AT activity assay (Fig. 3.27D). As expected, FabD preferred loading the elongation substrates Mal and MMal. Although turnover rates were significantly decreased to about $30 \%$ of the wild type activity, the absolute numbers of 25 to 30 molecules/s were still outstandingly high compared to PKS systems. 


\section{Results}

A

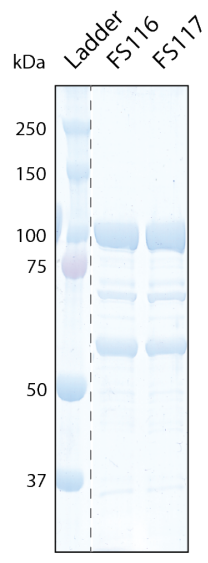

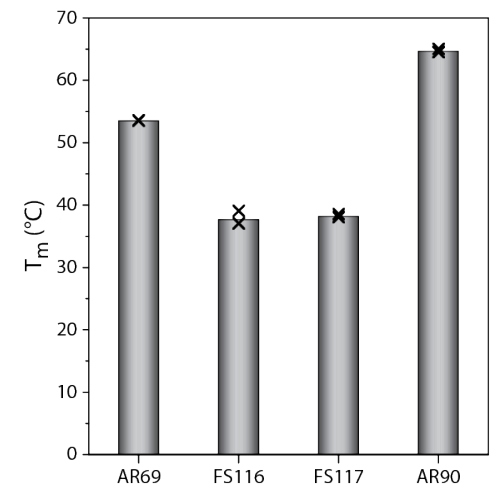

C

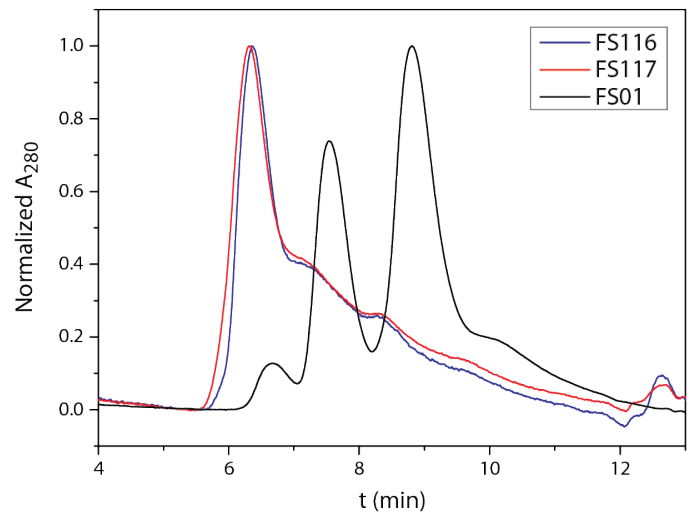

$\mathrm{D}$

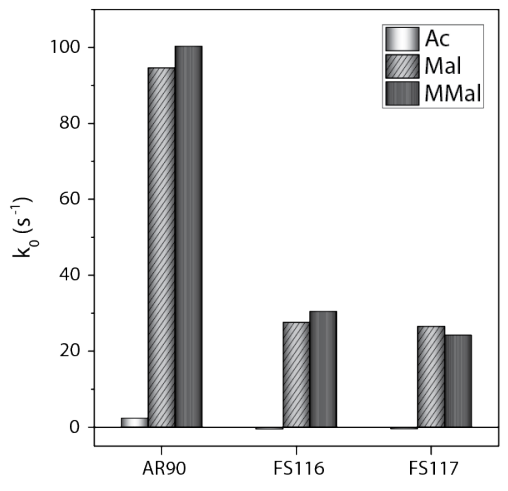

Figure 3.27: Analysis of KS-AT chimeras. (A) SDS-PAGE (10\% polyacrylamide) of mFAS/FabD chimeric KS-AT FS116 and FS117. Concentrated elution fractions after tandem affinity chromatography. (B) Average melting temperatures (in ${ }^{\circ} \mathrm{C}$ ) of mFAS KS-AT and E. coli FabD wild types (AR69 and AR90) and of chimeric KS-AT constructs (FS116, FS117) in storage buffer (Strep wash buffer pH 7.6). Measured in triplicates. Crosses correspond to one technical replicate (FS116, FS117) or one biological replicate (AR69, AR90). (C) Normalized size exclusion chromatogram of FS116 (blue) and FS117 (red) with DEBS3M5 KS-AT wild type FS01 (black) as reference. (D) Substrate screening of AT activity assay. Loading of substrates Ac (white), Mal (light gray, hatched), and MMal (dark gray) onto E. coli ACP mediated by wild type FabD (AR90) and by mFAS/FabD KS-AT chimeras.

Overall, analysis of proteins revealed their high potential in domain swapping approaches. Even though the chimeras suffered from artificial interfaces, leading to less stable and degraded protein, the transferase activity was still high. For our application as elongation module, interfaces could be optimized. Furthermore, I expect the integration of the type II ACP in the type I system to be challenging. The design of a suitable linker will be key for productive domain-ACP interactions. 


\section{Results}

\subsubsection{Generation of mFAS/DEBS3M6 KS-AT Chimeras}

Additionally to the new hybrids with FabD, we designed new chimeras with the AT domain from DEBS3M6, which is expected to be highly substrate specific for MMal-CoA. Overall, the first domain boundaries used seemed to be most promising, according to structural and sequential alignments, despite the low success with domain swapping of DEBS3 AT5. The same domain boundaries were used in a study of my colleague Mirko Joppe for domain swapping of mFAS AT in DEBS3M6. He showed that the exchange of solely the AT worked better than the exchange of LD-AT. Based on this data, we designed mFAS/DEBS3M6 KS-AT chimeras.

$\begin{array}{ll}\text { AR69 } & \text { mFAS(KS-LD-AT) } \\ \text { FS37 } & \text { mFAS(KS-LD)-DEBS3(AT)5 } \\ \text { FS118 } & \text { mFAS(KS-LD)-DEBS3(AT)6 } \\ \text { FS119 } & \text { mFAS(KS-LD)-DEBS3(AT)6 } \\ \text { AR265 } & \text { DEBS3(KS-LD-AT)5 } \\ \text { FS138 } & \text { DEBS3(KS-LD-AT)6 }\end{array}$

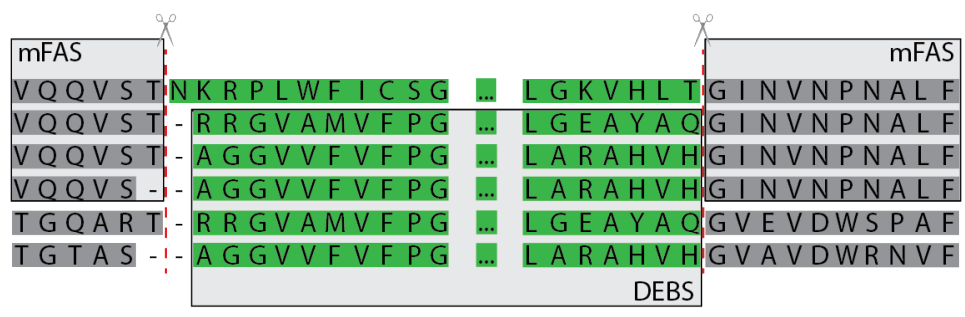

Figure 3.28: Domain boundaries for AT swapping with DEBS AT6. Two alternative boundaries were chosen for the first interface ( $m F A S(K A L)$ :DEBS3M6(AT)), leading to two chimeras FS118 and FS119.

We created a sequence alignment in order to find the corresponding domain boundaries in DEBS3M6 (Fig. 3.28). For one construct (FS118), we used the exact same domain boundaries as for swapping with DEBS3 AT5 (FS37). Since the wild type DEBS3M6 KS-AT does not contain the amino acid $\mathrm{T}$ at the $\mathrm{C}$-terminus of the $\mathrm{LD}$, we created a second chimera without this residue (FS119).

Table 3.11: Test expression yields of KS-AT chimeras after tandem affinity chromatography of $1 \mathrm{~L}$ expression culture.

\begin{tabular}{lccc}
\hline Protein & Pellet's Weight $(\mathbf{g})$ & Total Yield $(\boldsymbol{\mu g})$ & Yield $(\boldsymbol{\mu g})$ per g Pellet \\
\hline FS118 & 17.4 & 1270 & 73.0 \\
FS119 & 22.3 & 356 & 16.0 \\
\hline
\end{tabular}


Test expression of both proteins showed significantly higher yields for FS118, which contains the original domain boundaries for AT swapping (Tab.3.11). SDS-PAGE analysis of the concentrated elution fractions of FS118 and FS119 showed mainly full-length protein ( $95 \mathrm{kDa})$, but also degraded proteins (Fig. 3.29A). Proteins were further analyzed by TSA (Fig. 3.29B). FS118 showed low start fluorescence and gave a melting temperature of $41^{\circ} \mathrm{C}$ in storage buffer, which is significantly decreased compared to wild type mFAS KS-AT but comparable to wild type DEBS3M5 KS-AT (see section 3.4.2). FS119, on the other hand, showed high start fluorescence, which indicates unfolded protein, ${ }^{[224]}$ but gave a similar melting temperature of $40^{\circ} \mathrm{C}$. HPLC analysis of both constructs (Fig. 3.29C) fortified this finding. FS118 showed defined monomeric, dimeric, and higher oligomeric states (like the DEBS3M5 KS-AT wild type), whereas FS119 showed no defined dimeric species like the mFAS/FabD chimeric constructs FS116 and FS117 (see section 3.4.6). Overall, these results indicated that FS118 is a stable chimera and has great potential for its application in an mFAS assembly line, whereas FS119 is in general poorly stable and not suited. Even though proteins were not isolated in replicates, these preliminary results point towards the sensitivity of chimeras towards minimal changes (difference in one amino acid) in the artificial domain-domain interaction.

A

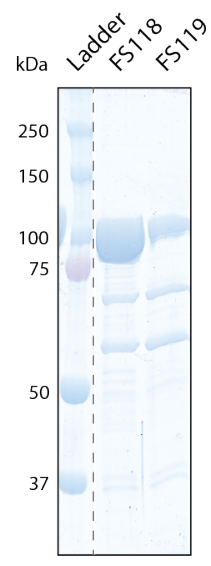

B

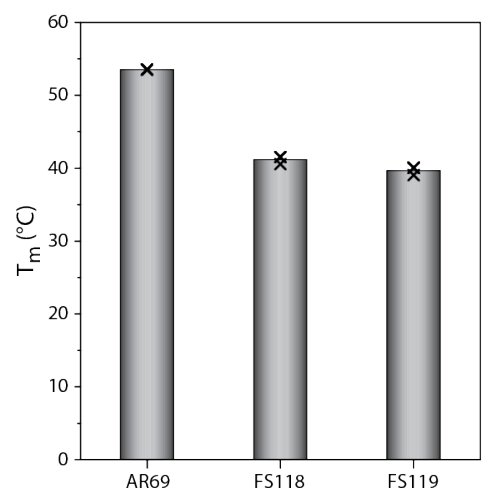

C

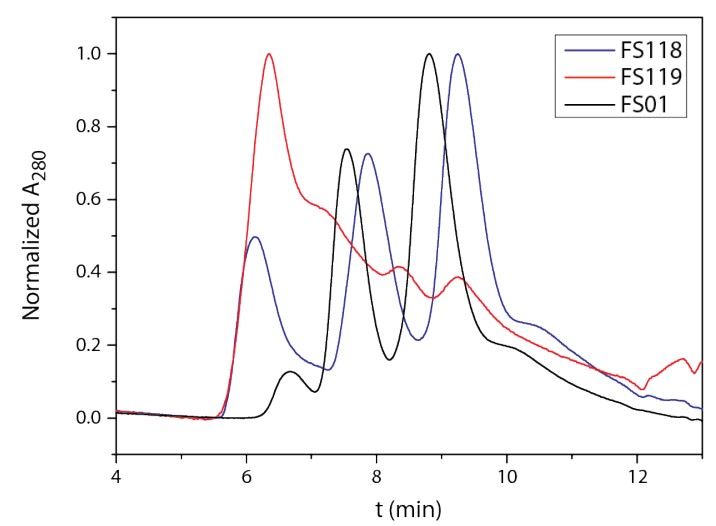

Figure 3.29: Analysis of chimeric KS-AT. (A) SDS-PAGE (10\% polyacrylamide) of mFAS/DEBS3M6 chimeric KS-AT FS118 and FS119. Concentrated elution fractions after tandem affinity chromatography. (B) Average melting temperatures (in ${ }^{\circ} \mathrm{C}$ ) of mFAS KS-AT wild type (AR69) and of chimeric KS-AT constructs (FS118, FS119) in storage buffer (Strep wash buffer $\mathrm{pH}$ 7.6). Measured in triplicates. Crosses correspond to one technical replicate. (C) Normalized size exclusion chromatogram of FS118 (blue) and FS119 (red) with DEBS3M5 KS-AT wild type FS01 (black) as reference. 


\subsubsection{Analysis of mFAS/DEBS3M6 KS-AT Chimeras}

Based on the promising results on expression and stability of the chimeric mFAS/DEBS3M6 KS-AT FS118, we started the master's thesis project of Alicia Just. In this project, we wanted to compare the AT activity of the chimera with the AT activity of the wild type system (FS138, DEBS3M6 KS-AT). Additionally to FS118, consisting of mFAS KS and DEBS3M6 AT domain, we designed the corresponding "inverse" chimera, consisting of DEBS3M6 KS and mFAS AT domain (FS139). The AT activity of the wild type mFAS KS-AT was characterized in kinetic detail before. ${ }^{[9]}$ Analysis of the three KS-AT constructs in their AT activity should give insights into the compatibility of mFAS/DEBS3M6 in domain swapping approaches.

Table 3.12: Average expression yields of KS-AT chimeras after tandem affinity chromatography of $1 \mathrm{~L}$ expression culture. Average of eight, five, and seven expressions for FS118, FS138, and FS139, respectively.

\begin{tabular}{lccc}
\hline Protein & Pellet's Weight $(\mathbf{g})$ & Total Yield $(\mathbf{m g})$ & Yield $(\mathbf{m g})$ per g Pellet \\
\hline FS118 & 9.14 & 0.913 & 0.0999 \\
FS138 & 8.77 & 4.96 & 0.566 \\
FS139 & 9.61 & 0.568 & 0.0568 \\
\hline
\end{tabular}

Alicia Just expressed all proteins as biological replicates under my supervision. ${ }^{[26]}$ Yields of both chimeras were drastically reduced compared to wild type yields, but still sufficient for AT characterization (Tab. 3.12). SDS-PAGE analysis showed mainly full-length protein of 95$98 \mathrm{kDa}$ (Fig. 3.30A), but revealed some degraded protein for the chimeras of around 70 and $60 \mathrm{kDa}$. TSA showed slightly decreased melting temperatures for both chimeras in storage buffer (FS118: $41^{\circ} \mathrm{C}, \mathrm{FS} 138: 43^{\circ} \mathrm{C}, \mathrm{FS} 139$ : $40^{\circ} \mathrm{C}$, Fig. 3.30B and S18). The wild type FS138 showed mainly dimeric species, whereas both chimeras formed monomers, dimers, and different higher oligomers in different ratios for biological replicates (Fig. 3.30C).

Table 3.13: Average expression yields of ACPs after affinity chromatography of $1 \mathrm{~L}$ expression culture. Yields determined from twelve independent expressions.

\begin{tabular}{lccc}
\hline Protein & Pellet's Weight $(\mathbf{g})$ & Total Yield $(\mathbf{m g})$ & Yield $(\mathbf{m g})$ per g Pellet \\
\hline mFAS ACP & 8.61 & 24.7 & 2.87 \\
DEBS3M6 ACP & 6.60 & 20.0 & 3.03 \\
\hline
\end{tabular}




\section{Results}

For AT characterization, mFAS and DEBS3M6 ACP were isolated in high yields (Tab. 3.13). ACP was further purified via SEC, resulting in highly pure protein (Fig. 3.30A). Different expression batches were pooled and full phosphopantetheinylation was confirmed by MS analysis (Tab. S9 and Fig. S19).

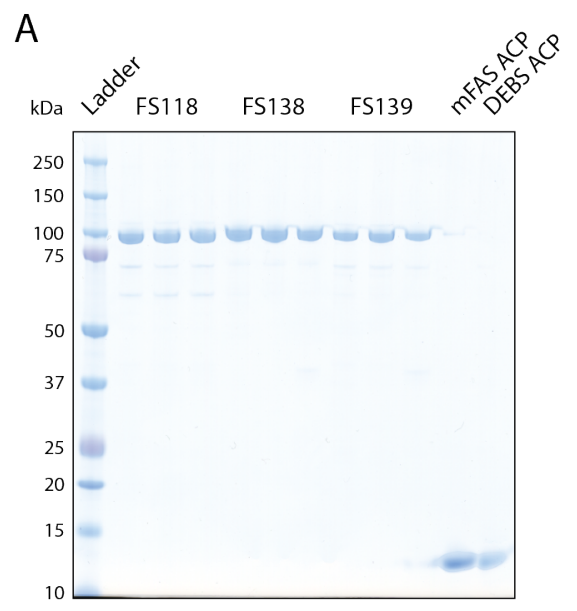

B

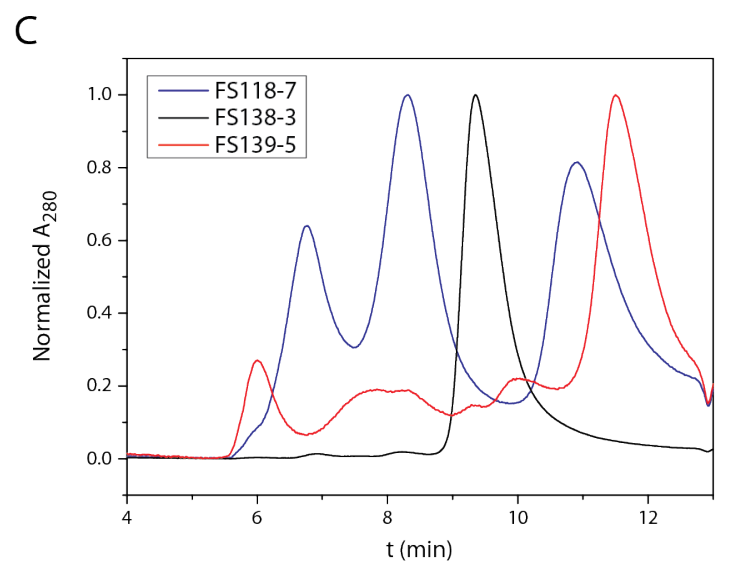

Figure 3.30: Analysis of chimeric KS-AT. (A) SDS-PAGE (NuPAGE Bis-Tris 4-12\%) of mFAS/DEBS3M6 chimeric KS-AT FS118 and FS139 and of wild type DEBS3M6 KS-AT FS138. Concentrated elution fractions after tandem affinity chromatography. (B) Average melting temperatures (in ${ }^{\circ} \mathrm{C}$ ) of mFAS and DEBS3M6 KS-AT wild types (AR69 and FS138) and of chimeric KS-AT constructs (FS118, FS139) in storage buffer (Strep wash buffer pH 7.6). Measured in triplicates. Crosses correspond to one technical (AR69 and FS118) and one biological replicate (FS138 and FS139). (C) Exemplary normalized size exclusion chromatograms of chimeras FS118 (blue) and FS139 (red) and DEBS3M6 KS-AT wild type FS138 (black).

In a first substrate screening, the loading of Ac, Mal, and MMal onto mFAS and DEBS3M6 ACP mediated by FS118, FS138, and FS139 was tested at fixed substrate concentrations (XCoA and ACP) of $100 \mu \mathrm{M}$ (Fig. 3.31A-C). As expected, the wild type FS138 loaded mainly MMal onto the native DEBS3M6 ACP, but was also able to load MMal onto the non-native mFAS ACP with slightly reduced turnover rates (Fig. 3.31B). The chimera FS118 (containing DEBS3M6 AT) also exhibited a loading preference of MMal and showed similar transacylation 
rates for MMal onto the DEBS3M6 ACP as the wild type and even higher rates for loading onto the mFAS ACP (Fig. 3.31A). The chimera FS139 (containing mFAS AT) overall showed the highest turnover rates, as expected, and loaded all three substrates onto its native ACP with higher rates for Ac and Mal than for MMal, like the wild type mFAS KS-AT (Fig. 3.31C). [9] This chimera was also able to load the three substrates onto the non-native DEBS3M6 ACP with only slightly reduced rates.

A

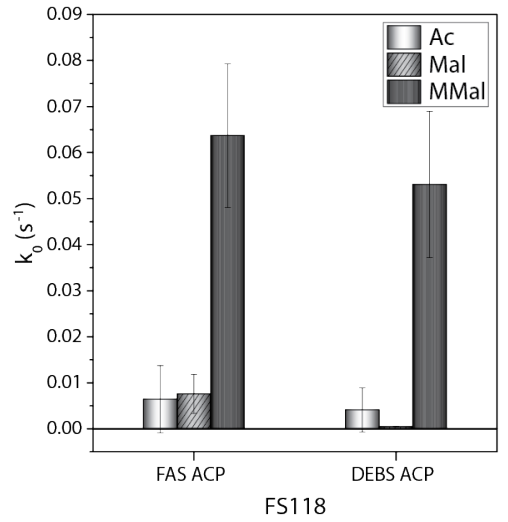

B

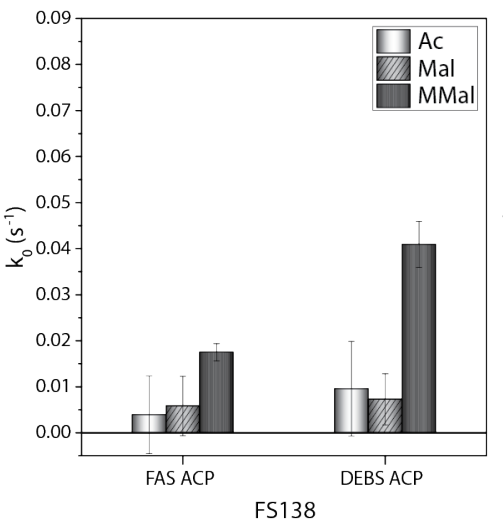

C

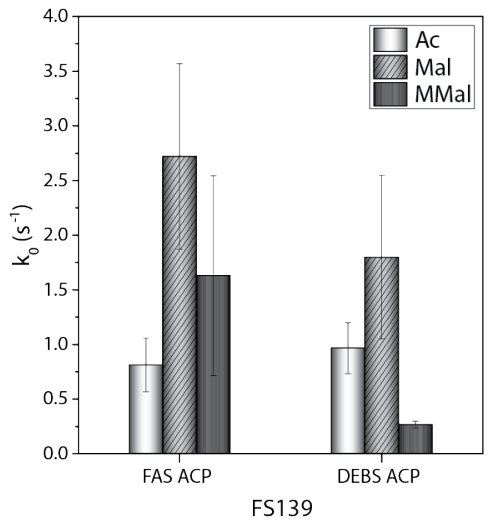

Figure 3.31: Transacylation substrate screening of KS-AT chimera FS118 (A), DEBS3M6 KSAT wild type FS138 (B), and KS-AT chimera FS139 (C). Substrates Ac (white), Mal (light gray, hatched), and MMal (dark gray) as well as mFAS and DEBS3M6 ACP were used at fixed concentrations of $100 \mu \mathrm{M}$. ATs measured in biological and technical triplicates. Bars show the average, error bars the standard deviation of each replicate.

Based on these first results, DEBS3M6 AT activity was analyzed in the chimeric construct FS118 and the wild type FS138 with its native substrates MMal-CoA and DEBS3M6 ACP (Fig. 3.32A and B). Biological triplicates were measured in technical triplicates and gave similar titration curves and kinetic parameters (Tab. S10 and Fig. S20 and S21). Kinetic parameters determined via global Michaelis-Menten fit for the average of each replicate are given in Table 3.14. Due to the relatively high value of $\mathrm{K}_{\mathrm{m}}^{\mathrm{MMal}-\mathrm{CoA}}$ for FS138, the ACP titration range was not ideal and the parameters are defective. Nevertheless, the data reveals that DEBS3M6 AT is relatively slow compared to other modular PKSs and features low catalytic efficiencies in ping and pong step. Overall, all kinetic parameters are decreased in the chimeric protein (turnover rate $70 \%$ of wild type and Michaelis-Menten constants $\mathrm{K}_{\mathrm{m}}^{\mathrm{MMal}-\mathrm{CoA}} 40 \%$ and $\mathrm{K}_{\mathrm{m}}^{\mathrm{ACP}} 37 \%$ of wild type parameters), leading to relatively similar catalytic efficiencies. Transition state energies are lowered by $1.3 \mathrm{~kJ} / \mathrm{mol}$ and $1.4 \mathrm{~kJ} / \mathrm{mol}$ during ping and pong step, respectively, in the chimeric protein compared to the wild type. This might simply be a consequence of the large calculation errors of the wild type kinetic parameters caused by a high value of $\mathrm{K}_{\mathrm{m}}^{\mathrm{ACP}}$. Overall, the data shows that we successfully designed a functional chimera, which shows only minor loss in AT activity compared to the wild type. 


\section{Results}

A

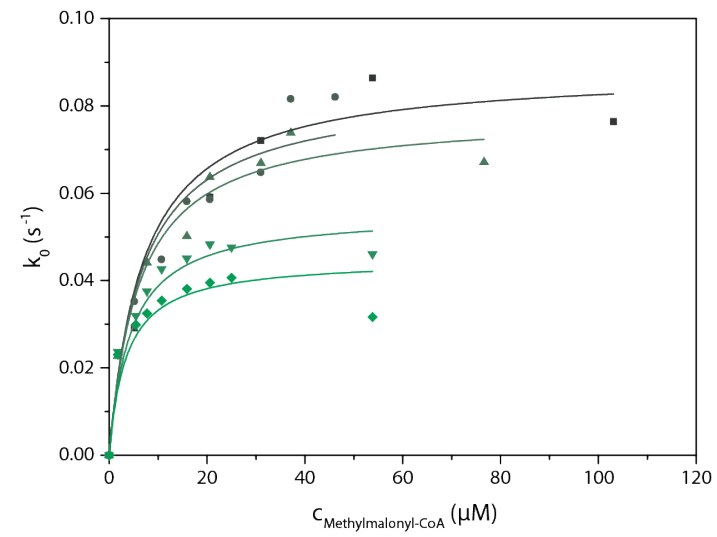

B

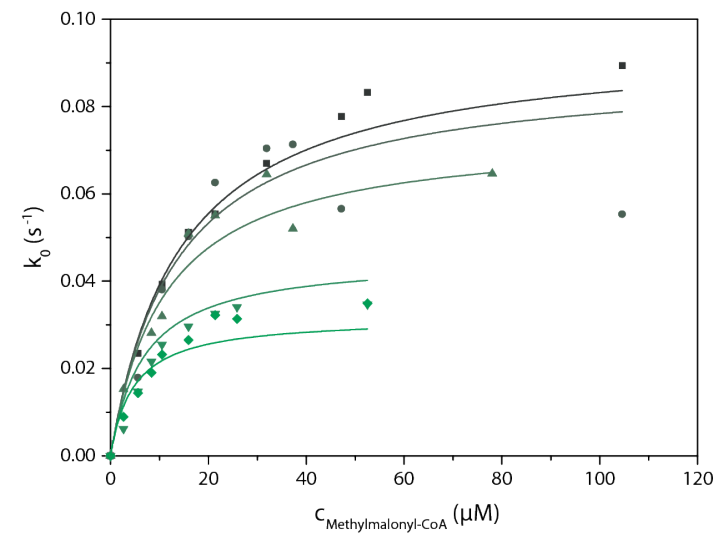

Figure 3.32: Titration curves of transacylation mediated by DEBS3M6 AT. Global fits of average measurements determined for biological triplicates measured in technical triplicates. (A) Chimera FS118. (B) Wild type FS138.

Table 3.14: Average parameters of transacylation of MMal-CoA onto DEBS3M6 ACP mediated by DEBS3M6 AT chimera (FS118) and wild type (FS138). Determined for biological triplicates measured in technical triplicates.

\begin{tabular}{|c|c|c|c|c|c|}
\hline Protein & $\begin{array}{l}k_{\text {cat }} \\
\left(\mathrm{s}^{-1}\right)\end{array}$ & $\begin{array}{c}\mathrm{K}_{\mathrm{m}}^{\mathrm{MMal}-\mathrm{CoA}} \\
(\mu \mathrm{M})\end{array}$ & $\begin{array}{l}\mathrm{K}_{\mathrm{m}}^{\mathrm{ACP}} \\
(\mu \mathrm{M})\end{array}$ & $\begin{array}{c}\mathrm{k}_{\text {cat }} / \mathrm{K}_{\mathrm{m}}^{\mathrm{MMal}-\mathrm{CoA}} \\
\left(\mathrm{M}^{-1} \mathrm{~s}^{-1}\right)\end{array}$ & $\begin{array}{c}\mathrm{k}_{\text {cat }} / \mathrm{K}_{\mathrm{m}}^{\mathrm{ACP}} \\
\left(\mathrm{M}^{-1} \mathrm{~s}^{-1}\right)\end{array}$ \\
\hline$F$ & $1.13 \times 10^{-1} \pm$ & . & 105 & $1.3 \times 10^{4}$ & $1.1 \times 10^{3}$ \\
\hline FS138 & $1.64 \times 10^{-1} \pm 2.28 \times 10^{-2}$ & $24.6 \pm 4.91$ & $257 \pm 66.2$ & $6.7 \times 10^{3}$ & $6.4 \times 10^{2}$ \\
\hline
\end{tabular}

We were not only interested in the functional, but also in the structural differences of wild type and chimera. In the course of her master's thesis, Alicia Just tried to solve the structures of FS118, FS138, and FS139 via X-ray crystallography under supervision of Dr. Apirat Chaikuad, Dr. Karthik Paithankar, and me. Unfortunately, in our numerous crystallization experiments, we were not able to obtain any protein crystals. ${ }^{226]}$ As further KS-AT crystallization experiments of master student Matthias Zeug showed, phosphate seems to form salt crystals and should be avoided in further experiments. 


\section{Discussion}

Parts of this chapter have been previously accepted for publication in: F. Stegemann, M. Grininger, "Transacylation Kinetics in Fatty Acid and Polyketide Synthases and its Sensitivity to Point Mutations", ChemCatChem (2021). [1] For individual contributions, copyright, and Creative Commons license, please see the statement of personal contributions.

\subsection{AT Activity Assay}

The core of the present thesis is the established AT activity assay, which is a powerful tool to analyze ATs in enzymatic detail. Our work demonstrates that this assay is extremely useful, especially for comparative studies on related proteins and for mutation studies. Using this assay, we were able to determine absolute kinetic parameters for both AT-mediated reaction steps (ping and pong) and to gain detailed mechanistic insights into functional differences of proteins from different classes or of different protein mutants.

This elaborate assay was established based on earlier work ${ }^{[133,211]}$ and was optimized during the course of my doctoral studies. Previous studies on AT activity performed single titrations for both substrates (X-CoA and ACP), titrating only one substrate while keeping the other substrate at saturated conditions. ${ }^{[14,133,211,227]}$ Separate Michaelis-Menten fits of both titrations gave access to all absolute kinetic parameters. This approach has the following disadvantage: ATs with a high value of $\mathrm{K}_{\mathrm{m}}$ cannot be analyzed by this assay, simply because substrate saturation cannot be reached. In our assay, we wanted to circumvent this problem and came up with the following solution: We simultaneously titrated both substrates by titrating the acyl substrate to a series of fixed ACP concentrations. During my master's thesis and in previous studies, ${ }^{[9,228]}$ these titration curves were fitted separately, leading to apparent kinetic parameters for each ACP concentration. These apparent kinetic parameters themselves were then fitted in double-reciprocal plots, giving access to global kinetic parameters. This approach has the following disadvantage: The absolute kinetic parameters are rather error-prone, since the data is not weighted equally. In the double-reciprocal fits, there is an antiproportional relationship between weight and ACP concentration, meaning that kinetic parameters determined for low ACP concentrations have higher weight than those determined for high ACP concentrations. This is particularly problematic, since the first are much more error-prone than the latter. In the present study, this problem was solved by globally fitting all data from all ACP concentrations via a global Michaelis-Menten fit (Eq. (3.14)). [212] This way, all data is weighted equally. Furthermore, this method is very robust to measurement errors and outliers and directly grants access to absolute kinetic parameters. 


\section{Discussion}

Overall, the AT activity assay is very valuable. However, some aspects need to be considered using this assay. It requires thorough handling of samples and is sensitive to even minimal handling differences. Usage of the same substrate batch (ACP and acyl substrate) for each system and for all mutants in our measurements reduced the variation caused by differences in substrate quality. Correct measurement requires careful inspection of raw data, ensuring the analyzed time span falls within the range of initial reaction velocity $v_{0}$. This is usually guaranteed for substrate consumption below $10 \%$. [129] Thus, for each measurement, substrate consumption was always examined. Additionally, dust and bubbles disturb proper measurement and working in a clean environment is essential. Wells were thoroughly inspected after careful and slow pipetting. Raw data was immediately inspected for outliers and artifacts and measurements were repeated, if necessary. Overall, the AT activity assay provides a large set of kinetic parameters, describing the AT-mediated reactions, with reasonable effort in the assay itself. Preceding preparation of stock solutions and high-quality protein, however, is quite time-consuming (biological triplicates of ATs, high amounts of high-quality ACPs) and acyl substrates are expensive. Adjustment of the originally 96-well format to the 384-well format downsizes the reaction volume to a fifth and by this reduces resources significantly (reduced usage of acyl substrates, enzymes, AT, and ACP, less protein preparations, more wells measured simultaneously).

Extensive usage of the AT activity assay in the present work also revealed general limitations. Kinetic parameters can only be determined precisely for systems with Michaelis-Menten constants lying within a certain range. For reliable values, the substrate (X-CoA and ACP) concentration range ideally should be varied within $0.2-5 \times \mathrm{K}_{\mathrm{m}}$. For extreme values, this range cannot be covered.

1. For exceptionally low Michaelis-Menten constants, low substrate (X-CoA or ACP) concentrations have to be used. This is problematic, since the substrate concentration needs to be significantly higher than the enzyme concentration (at least ten times). ${ }^{[129]}$ Decrease of the enzyme concentration is not infinitely possible, since poor signal-to-noise ratios impede proper data analysis.

2. On the other hand, exceptionally high Michaelis-Menten constants require high substrate (X-CoA or ACP) concentrations. This can lead to experimental problems, e. g. caused by substrate inhibition effects. Often the vast amount of ACP can simply not be provided and too high concentrated stock solutions can cause instability of ACP.

Nevertheless, our study demonstrates that even for systems with extreme Michaelis-Menten constants, kinetic parameters can still be estimated using this assay. 


\subsection{Kinetic Analysis of Acyl Transferases}

Using this assay, we characterized different ATs in their transacylation and hydrolysis activity. The initial substrate screening is a suitable and fast method to gain first insights into substrate tolerance and relative transfer velocities of different systems. Even though the absolute maximum velocities differ, the screening shows the same velocity trends: ATs from modular PKSs feature slower transacylation rates than those from iterative PKSs, which are slower than those from FASs (Fig. 3.4A and B). Hydrolysis mediated by FASs is significantly slower than hydrolysis mediated by PKSs.

Unfortunately, we were not able to express the standalone DEBS1 AT0 for analysis. The priming AT with C-terminal MBP fusion did not show any significant transacylation activity in numerous test measurements (Fig. 3.4C). Nevertheless, the hydrolysis for the native and the non-native priming substrates Prop and Ac were measurable. This indicates that the C-terminal MBP impedes productive AT-ACP interactions, but does not or at least not completely inhibit the acyl substrate loading onto the AT domain. AVES1 AT0, on the other hand, showed a significantly higher transacylation than hydrolysis rate already in the substrate screening (Fig. 3.4D). This system was chosen as a reference priming AT to DEBS and was not analyzed further due to the inactivity of the latter. In the following, only the elongation ATs will be compared regarding their activity.

Although limitations occured in hydrolysis measurements for many systems due to extreme Michaelis-Menten constants, the maximal turnover rate for all PKS systems was determined at least for the native substrate (Tab.3.1 and Fig. S7). All PKS systems feature similar hydrolysis rates, which is also in accordance with previous studies on other DEBS and PikA modules. ${ }^{[132,133]}$ Hydrolysis of FabD was not measurable.

Transacylation showed the highest turnover rates and efficiencies in both steps, ping and pong, for the FAS systems (Tab.3.2). The iterative PKS is faster and more efficient in both steps than the modular PKSs. Although only few kinetic studies on transacylation exist, the determined kinetic parameters are in good accordance with Michaelis-Menten constants and overall turnover rates determined for PKS systems. ${ }^{[133,143,206]}$ For FabD, there are numerous studies available, giving various and partly divergent kinetic parameters. ${ }^{14,211,216,227]}$ These studies determined even higher transacylation rates of up to 2000 molecules per second. Buffers with different phosphate concentrations and additives, $\mathrm{pH}$ values, and temperatures may influence the outcome of the parameters. ${ }^{[206]}$ Presence and absence of purification tags may also contribute to differences. Nevertheless, FabD was the fastest system in our setup with a turnover rate of almost 300 molecules per second (Tab. 3.2). The Michaelis-Menten constants we de- 


\section{Discussion}

termined are similar to the ones published by Misson et al. ${ }^{[14]}$ In the context of our study on FAS's and PKS's ATs, the kinetic parameters of the systems are comparable, since the measurements were performed under the same conditions. The only difference is that $E$. coli ACP contains solely a C-terminal His-tag for purification, whereas all other ACPs contain an additional N-terminal Strepll-tag. This additional tag could potentially affect the AT-ACP interaction, but would explain lower turnover rates for the other systems. 


\subsection{Implications of Kinetic Properties on the Acyl Transferase Reaction Mechanism by Comparing Hydrolysis and Transacylation}

Our enzyme kinetic analyses reveal that hydrolysis mediated by ATs of PKS systems is just one to two orders of magnitude slower than transacylation (Tab. 3.1 and 3.2), which is in accordance with previous studies. ${ }^{[133,143]}$ This rate difference is much less than for FAS systems for which four orders of magnitude difference were recorded. [9] We observe AT-mediated hydrolytic activity for all substrates - if native or non-native - transferred onto the ACP during transacylation and in most cases hydrolysis is fastest for the native substrates (Fig. 3.4). The purpose of the relatively high hydrolysis rates for PKSs remains elusive, but our data rules out any quality control by hydrolysis, as e.g. reported for some aminoacyl-tRNA synthetases in which hydrolytic active sites evolved in addition to their catalytic active sites. ${ }^{[13]}$

Comparing AT-mediated transacylation and AT-mediated hydrolysis can give insight into the substrate specificity of enzymes.

1. The PikAll AT5 is the only enzyme from our set of proteins for which we recorded a higher hydrolysis rate for the non-native substrate, although at low rate, speaking also in the specific case of PikAIII AT5 against a proofreading function. Different specificities for hydrolysis and transacylation are interesting from a mechanistic point of view, because they can just arise after the substrates have been loaded to the enzyme (after the ping step). Data implies that PikAllI AT5 can forward the native moiety MMal to the ACPloading pong step, while redirecting the non-native moiety Mal into hydrolysis. In this regard, PikAllI AT5 seems capable of differentiating between the two substrates in the pong step.

2. In case of the other modular PKSs (DEBS3M5 and RAPS3M14), the absence of hydrolytic activity for non-native substrates, with respect to the detection limit of the assay, indicates a more stringent selection for substrates in the ping step.

3. We note that, based on the catalytic efficiency of the ping step, we can calculate the Michaelis-Menten constants for the AT-mediated hydrolysis (Tab. S6). This is possible, because the efficiencies for the ping step in transacylation and for hydrolysis are identical (Eq. (3.18) and (3.24)). We observe decreasing $\mathrm{K}_{\mathrm{m}}^{\mathrm{X}-\mathrm{CoA}}$ values for hydrolysis from modular PKSs to the iterative PKS to the FAS system. This trend, as well as the trend in catalytic efficiencies (increased for iterative proteins), is overall similar to the ping step of transacylation. 


\subsection{Implications of Kinetic Properties on FAS/PKS}

\section{Function and Evolution}

A. Bar-Even and E. Noor et al. ${ }^{[229]}$ have mined enzyme databases for a systematic study on kinetic parameters of enzymes and substrates. As one of their key findings, they revealed that enzyme substrate pairs associated with primary metabolism tend to have higher turnover numbers than pairs of secondary metabolism and also average $k_{c a t} / K_{m}$ values are higher in primary than in secondary metabolism. We observe a very similar trend in our study on the transacylation function in FASs and PKSs; i. e., high turnover rates by FabD ${ }^{[14,216,227]}$ and MAT of FASs ${ }^{[9,230]}$ and low turnover rates by ATs of modular PKSs (Tab. 3.2). The lower turnover rates and catalytic efficiencies can be explained by serving the purpose of PKSs in providing sufficient efficiency to produce molecules at a low basal level or when necessary without draining vital metabolic pools (particularly in case of using Mal-CoA as elongation substrate). In contrast, the MAT domain of FAS needs to be fast and was under selection pressure for providing high rates for the high metabolic fluxes that are required by the central metabolic fatty acid biosynthesis. In conclusion, we observe in our dataset that the AT domains have evolved differently, serving the different purposes in enzyme function in the metabolic context. [231]

In previous studies, the lab of S. Smith and we described MAT from rat and mouse FAS as fast, substrate polyspecific, and plastic, ${ }^{[9,212,230]}$ which are typical properties of primordial enzymes. We speculate that in MAT primordial enzyme properties kept preserved due to its role in fatty acid synthesis and due to the KS-mediated condensation step ensuring the fidelity of the fatty acid biosynthesis. ${ }^{212]}$ Compared to MAT of FAS, the ATs of modular PKSs appear more specialized. Although ATs of modular PKSs with substrate tolerance towards mainly non-native elongation substrates have been reported, $[132,141,143,232]$ they have the ability to selectively transfer the native elongation substrates Mal and MMal, as we show in this study. Here, we note that we have not tested the transacylation of the native substrate ethylmalonyl-CoA.

Given that AT domains are integral part of monomodular iterative PKSs and modules of modular PKSs, their course of evolution is likely closely connected to the evolution of PKSs in general. Current models on the evolution of PKSs consider modular PKSs as successors of simpler systems that have likely been (also) iterative PKSs (and FASs or their precur-

sor). ${ }^{[6,42,44,78,233]}$ Accordingly, it is tempting to speculate that a primordial AT enzyme, to which MAT of FAS is still reminiscent in functional properties, has developed into more selective and slower modular AT domains (as they appear from the dataset of this study) as part of PKS evolution. 


\subsection{Impact of Interface Mutations on Transacylation Kinetics}

The critical domain-domain interaction between AT and ACP was further analyzed in a mutation study. Like for the wild type ATs, the initial substrate screening gave a first reliable insight into the effects of the mutations on reaction kinetics and revealed that the residue A539 is most likely not involved in the AT-ACP interaction (Fig. 3.7). Detailed kinetic analysis of mutant A539E confirmed this finding featuring wild type-like kinetic parameters (Tab. 3.3). In contrast to A539E, mutations in R850 led to intricate effects on both the AT-loading ping and the ACP-loading pong step.

1. The mutation R850K, preserving the positive charge, has almost no influence on the ATMMal-CoA interaction, as indicated by the ping step remaining unaltered in catalytic efficiency and transition state energy compared to wild type DEBS3M5 AT (Fig. 3.9). However, the pong step drops in efficiency and overall reduces turnover rates for transacylation ( $57 \%$ of wild type). The $\mathrm{K}_{\mathrm{m}}^{\mathrm{ACP}}$ of the pong step is similar to wild type AT, which indicates unchanged AT-X:ACP complex stability. Since the catalytic rate of the pong step $\left(k_{4}\right)$ is decreased, $K_{m}^{A C P}$ can just remain at wild type level when compensated by an increase of ratio $k_{-3} / k_{3}$ (see appendix "Mathematical Examination of Changes in Transacylation Kinetic Parameters Caused by Point Mutations"), indicating that the main effect of the mutation R850K is decelerating the formation $\left(k_{3}\right)$ and/or accelerating the (non-productive) dissociation of AT-X:ACP (k-3) (Fig. 4.1).

2. The mutant R850E inverses the charge from positive to negative and we expected severe changes in the kinetics of the transacylation reaction. Indeed, we observed lowest turnover rates within our set of mutants (Tab. 3.3). We note that the kinetic parameters of the pong step could only be collected with lower confidence due to the low $\mathrm{K}_{\mathrm{m}}^{\mathrm{ACP}}$. For a reliable estimate on $\mathrm{K}_{\mathrm{m}}^{\mathrm{ACP}}$, we therefore extracted data for saturated substrate MMalCoA concentration titrated with $\mathrm{ACP}$ and received a $\mathrm{K}_{\mathrm{m}}^{\mathrm{ACP}}$ of $3.46 \mu \mathrm{M}$ and an upper boundary of $4.6 \mu \mathrm{M}$ (Fig. S15). Given this data, we conclude that the pong step remains essentially unaltered in catalytic efficiency and transition state energy. In contrast, the ping step is severely affected by this mutation and responsible for the overall decrease of turnover rates. Since the catalytic rate of the ping step $\left(k_{2}\right)$ is contributing to $\mathrm{K}_{\mathrm{m}}^{\mathrm{ACP}}$, the drop of $\mathrm{K}_{\mathrm{m}}^{\mathrm{ACP}}$ is "just" a direct consequence of the slow ping step. Under this assumption, the kinetics of the pong step would be entirely unaffected in spite of exchanging the positively charged arginine with the negatively charged glutamate (Fig. 4.1). In conclusion, this mutant, designed for a severe impact on the domaindomain interplay during the pong step, mainly (or entirely) takes effect on the initial ping step, which demonstrates the intricate effect surface mutations can have. 


\section{Discussion}

3. In the mutant R850S, both ping step and pong step are affected by the amino acid exchange, as indicated by changed catalytic efficiencies and transition state energies compared to wild type DEBS3M5 AT, and effects cumulate to a decrease in turnover rates to $23 \%$ compared to wild type (Tab. 3.3 and Fig. 3.9). The decrease in the catalytic rates of the ping step $\left(k_{2}\right)$ and the pong step $\left(k_{4}\right)$ accounts for the increased stabilities of the complexes AT:X-CoA and AT-X:ACP (lowered $K_{m}^{X-C o A}$ and $K_{m}^{A C P}$ values), but ratios $k_{-} 1 / k_{1}$ and $k_{-3} / k_{3}$ may contribute here (Fig. 4.1). Overall, the effect of mutation R850S is not constrained to the pong step, but also invasive to AT-X formation; i. e., not showing the specific properties expected of an AT:ACP interface mutation.

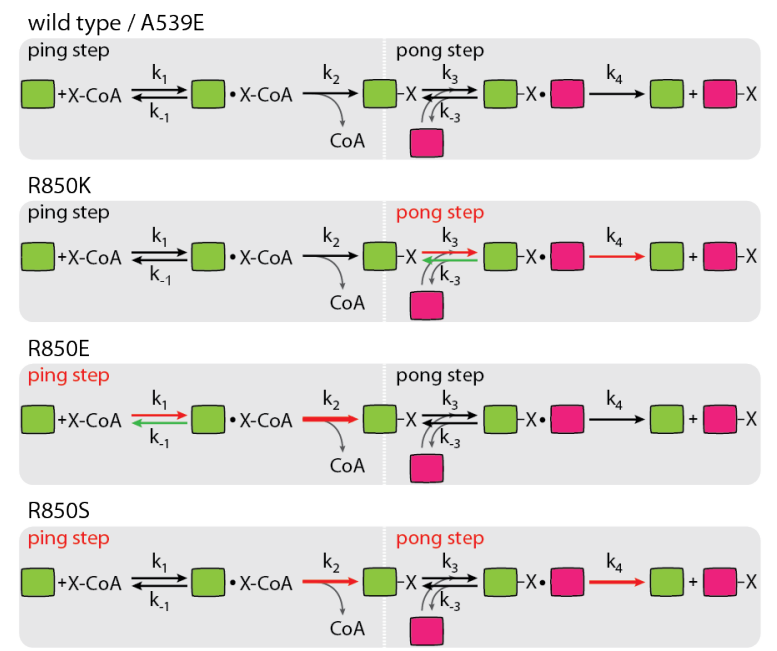

Figure 4.1: Plasticity of transacylation kinetics. Fluxes through the transacylation pathway in DEBS3M5 AT wild type and mutants. Green and red arrows indicate accelerated and decelerated reactions, respectively. The thicker the arrow, the more pronounced the effect. Only the most probable effects according to our interpretation are shown. For the mutant R850S, no statements can be given about changes in the kinetic constants $k_{1}, k_{-1}, k_{3}$, and $k_{-3}$ due to complex effects not covered by our mathematical examination (see appendix). Headers "ping step" and "pong step" are in red when decelerated. Domains AT and ACP are depicted as green and pink boxes, respectively. 


\section{Discussion}

\subsection{Implications of Interface Mutation Study on PKS Engineering}

Electrostatic networks have previously been shown to dominate interactions of ACP with catalytic domains. ${ }^{[14,221]}$ Accordingly, we assumed that R850 with its cationic guanidinium headgroup is likely responsible for a such a key electrostatic interaction at the AT:ACP interface. Further support for R850 being involved in AT-ACP interaction was received from $\mathrm{X}$-ray data in which residue R850 is poorly resolved in electron density in the KS-AT didomain structure (Fig. S22), ${ }^{[99]}$ indicating that R850 is not part of an electrostatic network of AT, but involved in AT-ACP interaction. Our kinetic data reflects the importance of the positive charge by the rather moderate effect of $\mathrm{R} 850 \mathrm{~K}$ mutation on the overall rate of transacylation compared to mutations R850S and R850E erasing and inverting the charge at this amino acid position, respectively. However, with help of the quantitative data, we likewise revealed that the mutations are not constrained to the AT-ACP interplay, but able to change kinetics of the entire transacylation reaction sequence (AT-loading ping step and ACP-loading pong step).

The incorporation of non-native elongation substrates to polyketides has previously been achieved by exchanging AT domains; i. e., replacing the native domain with a domain of interest. $[133,168,234]$ While such strategies can be successful in delivering the desired compound, the chimeric PKSs inherently suffer from non-cognate (permanent) interfaces in the KS-AT didomain as well as non-cognate (transient) interfaces during substrate shuttling (AT:ACP), which generally leads to compromised activity. ${ }^{[20]}$ Engineering domain-domain interplay can be useful when non-cognate domains are to be integrated in chimeric PKSs. Beyond the boundaries of the DEBS3M5 AT that has served as a model system in this study, the intricate impact of mutations on the catalytic properties suggests that simple engineering strategies based on local effects of amino acid exchanges are prone to fail. For example, engineering interfaces for stabilizing enzyme-substrate complexes (low $\mathrm{K}_{\mathrm{m}}$ values) will not necessarily lead to high turnover rates and high catalytic efficiencies. However, the design of chimeric PKSs that perform product synthesis at high efficiency can then be successful, when including detailed enzyme kinetic analysis, ideally complemented by computational methods (as recently demonstrated with FAS ${ }^{[235]}$ ) that eventually allows setting up highly effective enzyme kinetic processes. 


\subsection{Towards the Generation of a Functional Chimeric mFAS Module}

In order to convert the iterative mFAS into an assembly line, several engineering aspects need to be considered (Fig. 2.10).

1. The design of the loading function: One needs to ensure, that the ATs of priming and elongation modules are highly specific for their substrates and discriminate against the respective other substrate so that there is no crosstalk between priming and elongation.

2. Design of an elongation module: Proper scaffold design needs to ensure intramodular communication between the different domains and processing of the substrate throughout the whole module (chain elongation and processing).

3. Design of covalent and/or non-covalent linkers between the modules: Proper scaffold design needs to enable intermodular communication (chain translocation), favoring the vectorial over the iterative working mode.

Previous studies have shown that domain swapping approaches often result in unstable and inactive proteins or proteins with reduced activity. ${ }^{[6,20,206]}$ Problems may occur regarding all three engineering aspects. In theory, I would expect that the implementation of a new loading function and design of a chimeric elongation module work best with similar systems. KS-AT structures from mFAS and DEBS3M5 revealed a high similarity. ${ }^{[9,99]}$ Additionally, DEBS3 AT5 met the criteria for donor ATs. It showed a high specificity for MMal, discriminating against priming substrate $A c$ and elongation substrate $\mathrm{Mal}$, with sufficient transacylation rates of 1.3 molecules/s (75 molecules/min; Tab. 3.2). Thus, within our set of characterized ATs, this domain seemed to be ideally suited for swapping into mFAS to create an elongation module. To increase chances of successful chimera design and to ideally create two FAS-based modules specifically loading different elongation substrates, we additionally used the Mal-loading RAPS3 AT14, which shows a high sequence identity to mFAS and DEBS3M5 AT.

Even though structural information on mFAS and DEBS3M5 KS-AT as well as sequence alignments were used to find proper domain boundaries (Fig. 3.11), problems in scaffold design occured. Chimeric FAS constructs with DEBS3 (LD-)AT5 and RAPS3 (LD-)AT14 gave drastically low yields and showed protein degradation (Tab. 3.4 and Fig. 3.12). Although proteins were only isolated in single replicates, these preliminary results point towards the following trends. For the LD-AT swap, we designed two different chimeras - one following the sequence alignment, the other following the structure alignment. In both chimeras (with DEBS and with RAPS), the proteins with domain boundaries chosen based on structure alignment resulted in significantly higher yields than the proteins with domain 
boundaries based on the sequence alignment. Additionally, these constructs gave higher yields than the constructs with only the AT swapped. This might imply that the LD-AT swap creating an artificial KS:LD interface is better suited for domain swapping than the AT swap leading to an artificial LD:AT interface, which would be in accordance with a previous study. [20]

These initial results confirmed that the design of a chimeric elongation module is quite challenging. We decided to first focus on the proper design of the loading function. For this, we reduced the complexity of the chimera by working on the KS-AT subconstruct to establish suitable artificial domain:domain interfaces. In the following, we focused on the DEBS3M5 construct, since structural information is beneficial to identify problems in the chimeric constructs. We designed eight different KS-AT chimeras with adapted domain boundaries after Yuzawa et al.'s strategy to design active chimeras (Fig. 3.13). [20] They describe the boundaries of KAL-AT-PAL1 as universally applicable domain boundaries for AT swapping in most type I PKS modules. Expression and analysis of the chimeric didomains showed that these cannot be applied to mFAS (Tab.3.5 and Fig. 3.14). Modified domain boundaries did not significantly improve protein stability and led to rather lower yields. In general, the constructs with swapped LD-AT resulted in higher expression yields than constructs with swapped AT, which was already observed in test expression of the full-length mFAS. TSA and SEC analysis showed that the artificial interfaces introduced in the didomain have serious effects on protein stability, folding, and formation of quaternary structures.

These rather disappointing results made us question the suitability of both wild types for the construction of chimeras. To understand the native interactions within the wild type and to estimate their robustness, we decided to analyze the KS-AT wild types via a mutation study. We used different bioinformatics tools to identify important residues in the KS:LD and LD:AT interfaces. 12 mutants were analyzed in the wild type mFAS KS-AT and revealed an exceptionally high robustness of this protein towards mutations (Tab.3.7 and Fig. 3.16, 3.17, and 3.18). The residue D61 was found to be crucial for the KS:LD interaction in mFAS and alanine mutant D61A showed a drastical loss in stability compared to the wild type. Mutant 1457A, located in the KS:LD interface, showed only minor destabilization effects, while all other mutants behaved like the wild type. Structural analysis revealed D61's role in forming a salt bridge between the KS and LD. Electrostatic interactions have been shown to influence protein folding and stability. ${ }^{[236,237]}$ To date, there is a controversy whether close-range electrostatic interactions like salt bridges are stabilizing or destabilizing proteins. ${ }^{236]}$ However, our analysis reveals that only mutation of D61 and not mutation of its counterpart R429 cause drastic destabilization. This demonstrates that D61 has to be part of a complex electrostatic network not limited to the salt bridge, which dominates the intrinsic stability of the protein fold, just like shown above for protein-protein interactions. 


\section{Discussion}

Overall, these results demonstrate a high robustness of the mFAS KS-AT fold and imply that mFAS is in general highly suited for AT swapping approaches. DEBS3M5 KS-AT wild type, on the other hand, was very sensitive to any point mutations. All mutations introduced in the KS:LD and LD:AT interfaces led to drastically destabilized proteins (Tab.3.9 and Fig. 3.21). This analysis revealed that this protein is not robust at all and not well suited for AT swapping. Differences in the robustness of the KS-AT fold of mFAS and DEBS towards mutations might again be explained by their evolutionary stage. Our findings support the above-mentioned hypothesis that in mFAS primordial enzyme properties were preserved which is reflected in its high robustness (shown in the present thesis for the KS-AT fold) and plasticity (shown for the AT in ref ${ }^{[9]}$ ). [238]

Based on that, we decided to use different ATs for swapping into mFAS. Like our analysis of FabD showed, it is a highly efficient enzyme discriminating against the mFAS priming substrate Ac (Tab. 3.2 and Fig. 3.4). We designed two chimeras (differing in length by three amino acids), which behaved very similar in protein expression and analysis (Tab.3.10 Fig. 3.27). Although the chimeras showed destabilization, the AT activity substrate screening still showed approximately a third of wild type FabD activity in transferring Mal and MMal. As seen in the SDS-PAGE gel, the analyzed protein samples contained different species of degraded proteins that have been dragged along in the tandem affinity chromatography (Fig. 3.27A). Since FabD does not contain a Strep-tag, the samples should not include full-length FabD. Indeed, no band of the size of solely FabD was observed in the gel. The band of $60 \mathrm{kDa}$ could correspond to KS-LD, stemming from purification of heterodimers formed between full-length and truncated protein chains. Thus, I expect the degraded proteins to not contribute to the measured AT activity. Taking degraded protein of around 30\% in the sample (as judged from SDS-PAGE, Fig. 3.27A) into account, one could assume around $43 \%$ wild type activity of the pure chimera in the substrate screening, which is outstandingly high for destabilized proteins. This illustrates the high potential of FabD in domain swapping approaches.

In parallel, we designed a chimeric KS-AT construct with DEBS3M6 AT. My colleague Mirko Joppe showed that the mMAT swap into DEBS3M6 works better than the m(LD-MAT) swap. Our mutation study of the mFAS KS-AT wild type supports this finding. The results of the mFAS/DEBS3M5 chimera were not further considered. Due to the inherent fragility of the DEBS3M5 KS-AT wild type, chimeras were highly instable and gave drastically low yields which do not allow any conclusions regarding the general swapping strategy in mFAS. Thus, we designed two constructs for the AT only swap, which differed in length by only one amino acid (Fig. 3.28). According to test expression, this small variation in the domain boundary had a huge impact on the protein yield, which was significantly lower for the shorter construct (Tab. 3.11). Both proteins showed some degradation, but the longer construct showed defined 
oligomeric states in SEC, which was not the case for the shorter chimera (Fig. 3.29). The stable chimera contains the original domain boundaries, but results in twentyfold increased expression yield compared to the mFAS/DEBS3M5 KS-AT chimera. This illustrates that evolutionary close ATs from the same PKS can feature fundamentally different properties and that even small changes in domain boundaries can have a huge impact on the stability of chimeras.

Based on these promising results on protein stability, we wanted to analyze the chimera in its AT activity. For comparison, we also cloned and expressed the wild type DEBS3M6 (KS-LD-AT) and the "inverse" chimera DEBS3M6(KS-LD)-m(MAT). The initial substrate screening showed comparable transacylation rates for the DEBS3M6 AT-containing chimera and DEBS3M6 wild type onto the corresponding ACP domain (Fig. 3.31A and B). Transacylation onto the mFAS ACP was also observed for both constructs with even higher rates for the chimeric protein. This could be explained by the active mFAS KS in the chimera contributing to the rate. On the other hand, this could be caused by the large measurement errors or it could be that only the apparent transfer rates differ, caused by differences in Michaelis-Menten constants. Apparent and absolute $\mathrm{k}_{\text {cat }}$ could still be similar, which can only be determined in full kinetic analysis. As expected, the "inverse", mMAT-containing chimera featured overall higher transacylation rates onto the native ACP (Fig. 3.31C). Transfer rates onto the non-cognate DEBS3M6 ACP were in a similar range. This data shows that usage of both ACPs, cognate to $\mathrm{KS}$ or cognate to AT, might be possible in the full-length mFAS module. Tolerance of non-cognate ACPs was also reported for PKS's ATs before, ${ }^{[20,142]}$ whereas another study showed drastically decreased transacylation activity of DEBS2M3 AT with DEBS3M6 ACP. [133] A study on DEBS2M3 KS showed that some ACPs from different modules are comparable substrates to ACP3, while others are not. [239] This could also apply to $\mathrm{mFAS} \mathrm{KS}$.

A detailed kinetic analysis was performed for DEBS3M6 KS-AT wild type and m(KSLD)-DEBS3M6(AT) chimera with the substrates MMal and DEBS3M6 ACP (Tab.3.14 and Fig. 3.32). Transacylation rate of the chimera was still at $70 \%$ wild type rate, which represents a huge success. Interestingly, both Michaelis-Menten constants are lowered in the chimeric construct. This means that for ping and pong step, the ratio of non-productive dissociation/formation of the enzyme-substrate complex is lowered (accelerating the formation and/or decelerating the non-productive dissociation). Self-acylation of mFAS KS with MMal (as demonstrated for the $\mathrm{m}(\mathrm{KS}-\mathrm{AT}-\mathrm{ACP}$ ) construct) may contribute to kinetic parameters. 


\section{Discussion}

Comparing kinetic parameters of DEBS3M5 and M6, transacylation rates in M6 were ten times slower than in M5 (Tab. 3.14 and 3.2). These slower transacylation rates are realistic, since the full-length DEBS was described with an overall slow production rate of 0.5 molecules $/ \mathrm{min}$. [16] The Michaelis-Menten constant $\mathrm{K}_{\mathrm{m}}^{\mathrm{MMal}-\mathrm{CoA}}$ for $\mathrm{M} 6$ is in the range for bacterial metabolite level, ${ }^{[219]}$ but tenfold higher in M5. The data on M6 indicates a control mechanism for regulating MMal uptake in M5. The Michalis-Menten constant $\mathrm{K}_{\mathrm{m}}^{\mathrm{ACP}}$, on the other hand, is 3.5 times higher in M6 than in M5. This moderate variation was also oberserved for other modular PKSs and the pong step should still run at maximum rate (saturated ACP conditions). Overall, kinetic parameters determined for M6 are in good accordance with parameters determined for M3. [133] This study showed that ATs of the same PKS may differ significantly in their kinetics.

Unfortunately, we were not able to solve the structures of chimeras and DEBSM6 KS-AT wild type. It would be very interesting to evaluate whether structural differences occur and if so, whether they can explain the variation in activity between the chimera and wild type. Furthermore, it would be helpful to compare structures of DEBS3M5 and M6 KS-AT, especially since the same domain boundaries were suitable for the design of chimeras with M6 but not with M5.

To understand differences in the success of chimera design with different donor ATs in more detail, I calculated the interfacial contact potential of the available wild type structures using PyMOL. Electrostatic interactions are important for the stability of proteins. ${ }^{236,237]}$ In this discussion, non-specific interactions like hydrophobic interactions are not included.

The contact potential of mFAS and DEBS3M5 in their KS:LD and LD:AT interfaces are shown in Figure 4.2 and 4.3. For both systems, the contact between LD and AT domain seems to be tighter than the one between KS and LD. While a wide cleft can be seen between the KS and LD which seems to be mainly held together by only some selective interactions, the LD-AT contact seems to hold more close interactions across a wider area. This may point out that LD-AT swaps are in general more suited than solely AT swaps. Comparing both interfaces from both systems, it becomes obvious that the main regions of interaction do not fit to the respective other system (repelling interactions). There are more contacts of high electrostatic potential involved in the domain-domain interactions of DEBS3M5 than in the ones of mFAS. This may explain the finding that DEBS3M5 is not robust to any point mutation. 


\section{Discussion}

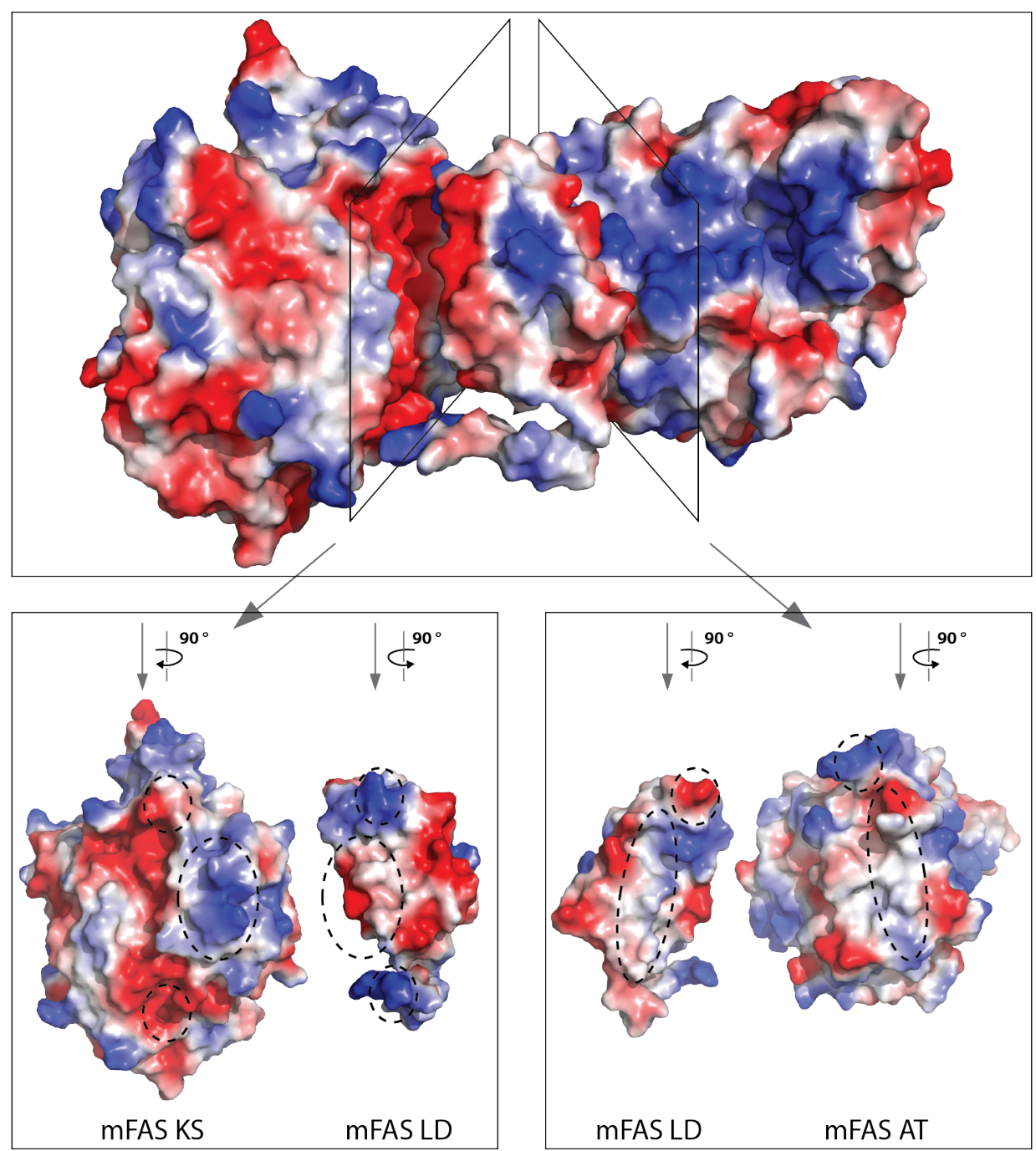

Figure 4.2: Contact potential of mFAS KS-AT wild type (PDB: $\left.5 M M^{[9]}\right)$. KS:LD and LD:AT interfaces are shown on the bottom. Red indicates negative potential, blue indicates positive potential. White indicates a neutral potential. Regions of interactions are highlighted.

To analyze differences in domain swapping with DEBS3M5 and DEBS3M6 AT, I tried to identify the residues in DEBS3M5 disturbing the $\mathrm{m}(\mathrm{LD})$ :DEBS3M5(AT) interface. Especially the upper interaction region of negative contact potential in DEBS3 AT5 (depicted in red) is problematic concerning the interaction with mFAS LD (Fig. 4.3). The lower region of negative contact potential may impair the interaction as well. I identified residue D814 in the upper region and residue D840 as well as the backbone formed by residue A857 in the lower region. Sequence alignment of DEBS3M5 and M6 showed the conservation of sites D840 (D2302 in M6) and A857 (A2314 in M6), but D814 of M5 is replaced by A2281 in M6. This residue variation could explain the significantly different behavior in domain swapping of DEBS3M5 and M6 AT. Introducing the mutation D814A in the mFAS/DEBS3M5 chimera could confirm or disprove this hypothesis. 


\section{Discussion}

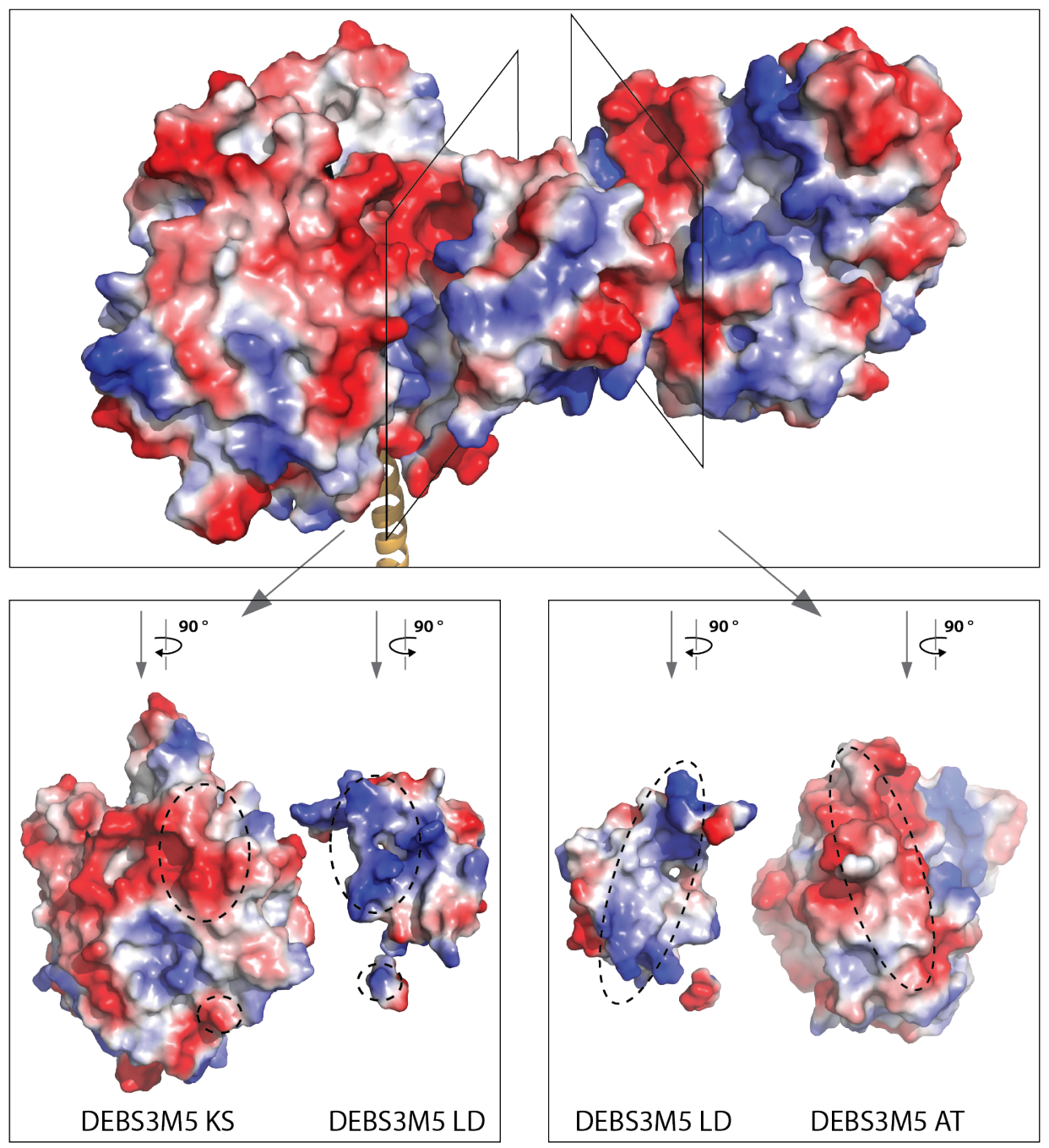

Figure 4.3: Contact potential of DEBS3M5 KS-AT wild type (PDB: $2 \mathrm{HG} 4{ }^{[99]}$ ). KS:LD and LD:AT interfaces are shown on the bottom. Red and blue indicate negative and positive potentials, respectively. White indicates a neutral potential. Regions of interactions are highlighted. 


\section{Discussion}

FabD/mFAS chimeras showed destabilizing effects, but nonetheless AT activity (Fig. 3.27). Since FabD features oustandingly high transferase efficiencies (Tab. 3.2), these chimeras have a high potential for usage in an mFAS-based assembly line. Even though FabD and mMAT are overall similar (Fig. 4.4A), regarding the interfacial contact regions, it became obvious that mFAS LD and FabD are not compatible (Fig. 4.4B). Especially the lower region of FabD featuring the negative potential might hamper the interaction with LD leading to a loss of stability. This may be a good starting point for mutagenesis in order to improve the artificial domain-domain interaction. For introducing FabD in an mFAS module, the design of a proper AT-ACP linker is key to success.
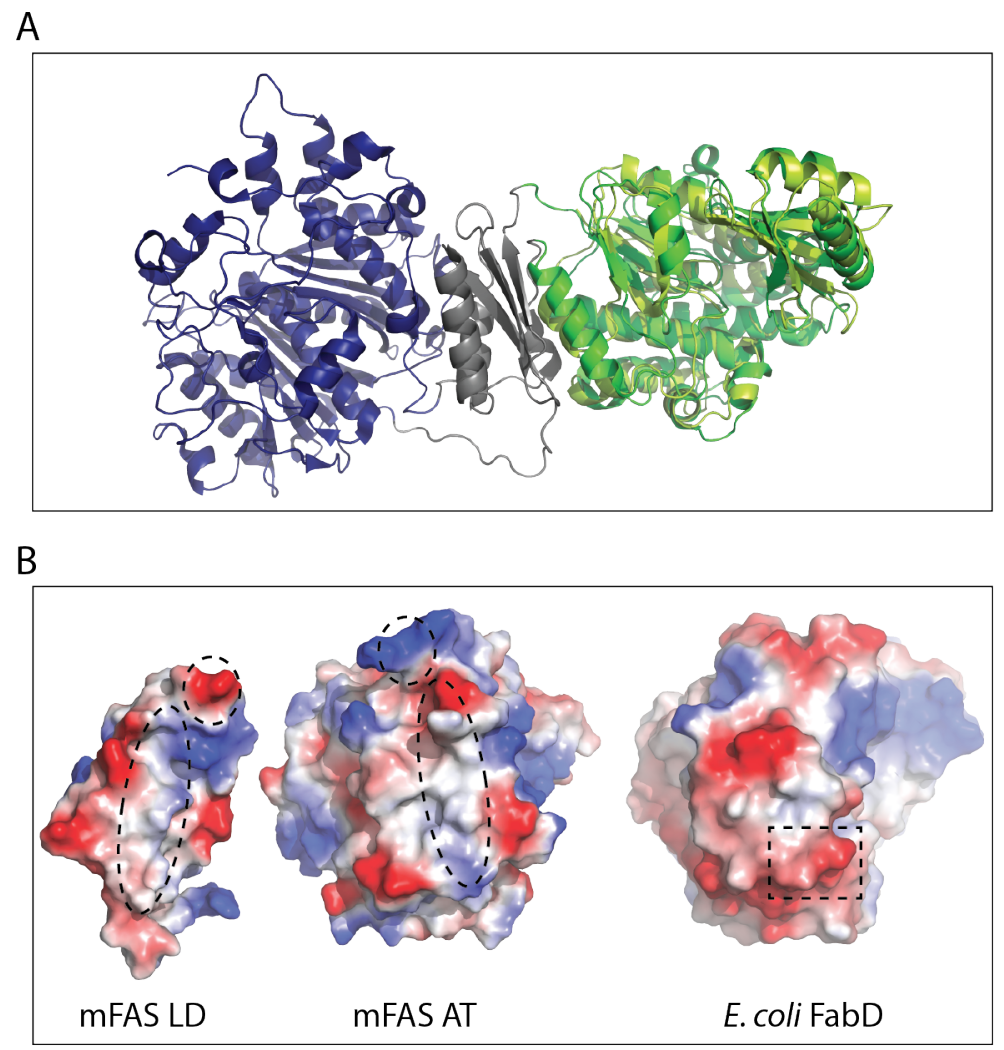

Figure 4.4: Analysis of FabD (A) Cartoon representation of mFAS KS-AT (PDB: 5MY0 ${ }^{[9]}$ ) and E. coli FabD (PDB: 1MLA ${ }^{[136]}$ ). Alignment of mMAT (green) with E. coli FabD (lemon) demonstrates their structural similarity $(\mathrm{RMSD}=2.823 \AA)$. mFAS KS and LD shown in blue and gray, respectively. (B) Contact potential of mFAS LD and AT and FabD. Regions of LD:AT interactions are highlighted in mFAS. Region of repelling interaction is highlighted in FabD. Red and blue indicate negative and positive potentials, respectively. White indicates neutral potential. 


\subsection{Experimental Outlook: Implementation of Chimeras in mFAS Modules}

At the end of my doctoral studies, Lynn Buyachuihan and I tried in a collaborative project to implement the active chimeric mFAS/DEBS3M6 KS-AT in an elongation module. To reduce complexity, we decided to use the minimal elongation module consisting of KS-AT-ACP. Activity of stable chimeras could then be analyzed in a minimal assembly line by following the production of a TAL derivative. The wild type full-length mFAS produces around $0.10 \mathrm{TAL} / \mathrm{s}$ (6.4 TAL/min), whereas the $\mathrm{m}(\mathrm{KS}-\mathrm{MAT}-\mathrm{ACP})$ construct is faster with around $0.28 \mathrm{TAL} / \mathrm{s}$ (16.5 TAL/min). ${ }^{[17]}$ Given the slow transacylation rate of the chimeric KS-AT of around $0.11 \mathrm{MMal} / \mathrm{s}$ (6.6 MMal/min; Tab.3.14), the transacylation reaction will probably limit the overall TAL derivative production rate significantly (maximal rate possible is around $0.055 \mathrm{TAL}$ derivative $/ \mathrm{s}=3.3 \mathrm{TAL}$ derivative $/ \mathrm{min}$ ).

Nevertheless, to see if the chimera can be installed in the elongation module (scaffold design), we designed two FAS modules with DEBS3M6 AT, based on the active chimera FS118 - one containing mFAS ACP, the other one containing DEBS3M6 ACP. Since the slow DEBS3M6 AT might not be well suited for efficient product formation, we decided to create further chimeric modules with different elongation substrate-specific ATs. Functional analysis of the modular venemycin PKS revealed relatively high turnover rates, which contains Mal-transferring ATs. ${ }^{[206]}$ Another study found FAS-like PKSs in sacoglossans and showed the substrate specificity for MMal of ATs from Elysia chlorotica PKS1 (EcPKS1). ${ }^{240]}$ With its close evolutionary relationship to FASs, we expected its AT to be faster and more efficient than those from modular PKSs. Thus, we designed minimal FAS modules with swapped AT and swapped LD-AT domains from VemG, VemH, and EcPKS1 with the same domain boundaries as in FS37/FS118 (AT swapping) and FS38 (LD-AT swapping).

In a first approach, we decided to use the ACP from the PKSs and not from mFAS in the elongation module. This is especially promising for the venemycin chimeric constructs, since VemG and VemH naturally contain only the minimal set of domains. In doing so, only one artificial interface is installed in the elongation module. We tested their expression and isolation via tandem affinity chromatography. Our results showed that the stable chimeric subconstruct cannot be easily installed in the elongation module. Both mFAS/DEBS3M6 constructs showed low expression yields. Only one of the in total nine different elongation modules, mFAS(KS)EcPKS1(LD-AT-ACP) (FS172), showed good stability and quality in our initial experiments (proper scaffold design). 


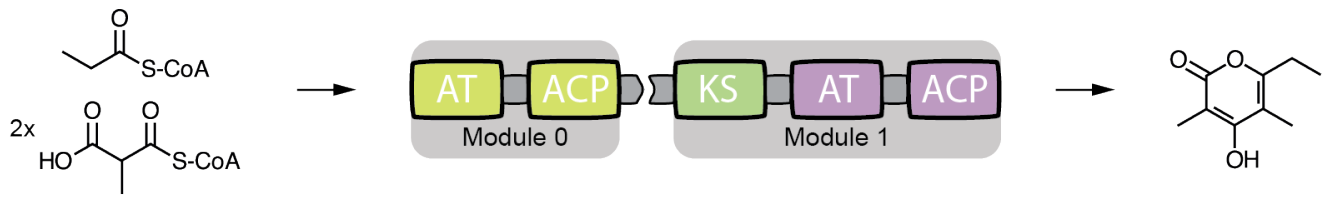

Figure 4.5: Assembly line consisting of a Prop-loading priming module (Module 0 ) and the MMal-loading chimeric elongation module (Module 1). Module 0 (depicted in yellow) is the DEBS priming module. The elongation module consists of mFAS KS (green) and of AT-ACP from EcPKS1 (purple). Modules are non-covalently linked using DEBS3M4/5 DDs.

This module with the MMal-loading AT from EcPKS1 was analyzed with an established priming module ${ }^{[209]}$ in a minimal assembly line (Fig.4.5). After loading of one priming and two elongation substrates, spontaneous cyclization/lactonization occurs leading to the release of a TAL derivative, which can be followed and quantified by absorption at $298 \mathrm{~nm}$. Our initial experiments showed that we successfully eliminated the loading of the priming substrate Prop by the chimeric elongation module, but only minor TAL derivative production rates were detected. Different control experiments proved that the non-native substrates Prop and MMal are tolerated. All three above-mentioned engineering aspects could be cause of the low production rates. Further experiments on different parts of the assemby line will be needed to distinguish between the mentioned possibilities and to identify the cause of the slow production rate.

1. To analyze if the AT domain itself is limiting the TAL derivative production rate, the wild type EcPKS1 and chimera KS-AT should be cloned, isolated, and analyzed in the AT activity assay with mFAS and EcPKS1 ACP. Even the substrate screening is sufficient for first insights into transfer rates.

2. An intramodular communication problem could arise through the non-cognate (permanent) $m(K S)$ :EcPKS1(AT) interface as well as through the non-cognate (transient) $m(K S)$ :EcPKS1(ACP) interface. Experiments on a chimeric KS-AT construct as well as exchange of the EcPKS1 ACP domain by the mFAS ACP could be performed.

3. Intermodular communication problems could be caused by an additional Strepl-tag upstream to the DD5, which might impede the formation of the coiled-coil structure to link the priming and elongation module. Previous studies showed the acceptance of the N-terminal His-tag downstream to DD4. $[163,199,209]$ To ensure proper linkage of the modules, the purification tag could be removed, the DDs could be replaced by another pair of non-covalent linkers (SYNZIPs), ${ }^{[163]}$ or the two modules could be fused together on one polypeptide chain.

a) Removal of the purification tag: Previous experiments showed problems with an alternative purification strategy via His-tag affinity, ion exchange chromatography, 


\section{Discussion}

and SEC. Thus, the Strepl-tag needs to be removed after isolation, adding another purification step to the protocol.

b) Replacement of DDs: Since a previous study has demonstrated the efficient linkage of two modules via the DDs, ${ }^{[209]}$ I would not expect them to be the bottleneck in intermodular module communication. However, different non-covalent linkers like SYNZIPS could be tested.

c) Fusion of modules: This approach has the advantage that the two modules are forced to interact with each other. Nevertheless, there are two big disadvantages: First, the size of the fusion construct is quite problematic and even lower (or zero) yields would be received. Second, by fusing the two modules we will no longer be able to perform different control measurements with only one of the two modules. We would no longer be able to distinguish between different reactions and to trace back experimental problems to their root.

Overall, we made tremendous effort to realize the idea of an mFAS-based elongation module. We followed different approaches and used different strategies to tackle various problems. Even though we did not achieve this challenging aim yet, we have taken essential steps towards an mFAS-based assembly line. Self-priming by the chimeric elongation module was significantly suppressed and minor amounts of the expected TAL derivative were produced. I will discuss the different strategies in the following. 


\subsection{Strategies for Creating Functional Chimeras}

Our initial strategy for the design of a functional FAS module was to use deeply characterized ATs for domain swapping in full-length mFAS. Key steps of this strategy are

1. identification of suitable donor ATs and

2. comparison of stability and activity of wild type and chimera.

A suitable donor AT is necessarily specific with respect to the elongation substrate, ideally efficient, and related to the acceptor system. Following this strategy, we made use of our extensive kinetic study on ATs. Based on structural information, we designed chimeras with DEBS3M5 and RAPS3M14 AT, which unfortunately all showed poor stability (Tab. 3.4 and Fig. 3.12).

After the limited success of the initial chimeric full-length mFAS, we chose to modify the strategy by reducing the complexity of the protein to the essential KS-AT subconstruct. This construct is much easier to modify and shows significantly higher yields. Thus, effects on protein stability can be identified more sensitively by analyzing differences in protein yields. After establishing proper artificial domain boundaries in the subconstruct, the chimera can in principle be implemented in the full-length module. The first experiments confirmed that domain swapping is challenging and fine-tuning might be needed. Thus, we decided to investigate numerous domain boundaries with focus on the DEBS3M5 AT, since structural information can support finding suitable interfaces. However, using the less complex subconstruct did not improve domain swapping success rates. We were not able to gain stable chimeras (Tab. 3.5 and Fig. 3.14). Thus, we had to take a step back and analyze the wild types to evaluate their compatibility for domain swapping. After detailed analysis via a mutation study to improve the domain boundaries, we finally came to the conclusion that the donor AT is not suited for domain swapping into mFAS due to its fragility towards mutations.

After this time-consuming and elaborate approach, we decided to create chimeric KS-AT constructs using known substrate-specific ATs as donor without their prior analysis. To save time, the donor AT was analyzed in kinetic detail after the design of stable chimeras in both wild type and chimera in parallel (Tab.3.14). Following this strategy, we were able to create a stable and functional chimeric KS-AT with DEBS3 AT6 which showed only minor loss of transacylation activity, but overall low kinetic rates. Unfortunately, the donor AT itself features low transacylation activity. Thus, the active chimera was of limited value and not suited for the efficient biosynthesis of designer products. 


\section{Discussion}

Nevertheless, we tried to implement this active chimera as proof of principle in a minimal assembly line consisting only of a priming and an elongation module. We combined this experiment with the strategy of directly creating minimal FAS-modules with other donor ATs. We used the original domain boundaries for AT and LD-AT swap and chose AT domains from 1. venemycin synthase and from 2. EcPKS1.

1. We decided for two ATs from the venemycin synthase due to product rates of 0.6 molecules/s (36 molecules/min), which is quite high for modular PKSs. ${ }^{206]}$ The venemycin PKS contains only two elongation modules. Assuming that transacylation is the slowest process in biosynthesis, transacylation rates have to be at least around 1.2 molecules/s (72 molecules/min).

2. EcPKS1, a sacoglossan PKS, is evolutionary close to FASs. ${ }^{240]}$ No information on production rates is available, but our kinetic analysis and fundamental findings from $A$. Bar-Even and E. Noor et al. ${ }^{[22]}$ suggest that this close relationship might accompany with higher turnover rates. The chance of successful design of a stable chimera might even be increased by the similarity of the systems.

Indeed, this strategy yielded a stable chimera with EcPKS1 (FS172) (proper scaffold design of elongation module). Three of the eight other chimeras were only expressible with low yields (among them two mFAS/DEBS3M6 chimeras), the others were not isolated at all (poor scaffold design). Among the three expressible chimeras, there was also one chimera which differed only in one amino acid in the KAL (one amino acid less than in FS172). This, again, points towards the fragility of proper domain boundaries in chimeras. In order to evaluate the design of the loading function, the stable chimera was tested in a minimal assembly line consisting of a priming and the chimeric elongation module. The low activity in producing the TAL derivative in this setup can be caused by all three engineering aspects (design of the loading function, of the elongation module, of intermodular linkers) and one cannot clearly distinguish between them. I assume that the problem is most likely caused by the installation of the loading function. Analysis of the elongation module showed good stability and we made use of an established priming module with established intermodular linkers in our setup. The additional purification tag might impede the linkage. To get to the bottom of this, kinetic analysis of the AT activity in the wild type and the chimera is essential. 


\section{Discussion}

All three strategies are quite elaborate and demonstrate that problems can occur in each step of the assembly line design. Problems on later stages without prior establishment of the earlier steps often require to take a step back in order to identify the root of the problem. In my opinion, the best approach would be the stepwise increase of complexity - starting from analysis of donor ATs in robustness and activity, vii over creating and investigating chimeric subconstructs prior to installation in elongation modules to the final assembly line. This strategy has the huge advantage that the cause of each problem can be identified more easily and each problem can be tackled separately. Changes on early levels are less time-consuming and resources are not wasted on ATs which are simply not suited for our approach. In fact, the last strategy of directly creating a chimeric elongation module was most successful, but only because it was build on the extensive groundwork of the others. However, it forced us to go back to the most basic level of wild type characterization.

viiEven the initial substrate screening is able to provide valuable information and allows to draw conclusions on relative activity, while saving time and cost compared to full kinetic analysis. 


\section{Discussion}

\subsection{Bioinformatics Tools in Chimeric Protein Design}

We used different simple bioinformatics tools for the identification of interfacial residues and for estimation of mutational effects on the protein's energy. As expected, Rosetta, being the most elaborate tool among these, gave the most reliable output. Clear correlation between predictions and experimental data on the mFAS KS-AT wild type was recognizable for chain A and chain D (Fig. 3.22, 3.23, and 3.24). We chose both chains as they featured a high RMSD, but both had the highest quality in the structural solution (PDB: $5 \mathrm{MY0}{ }^{[9]}$ ). In retrospect, it would have been useful to analyze chain C (the chain with the lowest quality) as well, using Rosetta. For DEBS3M5 KS-AT, on the other hand, no correlation tendencies were observed (Fig. 3.25). This can be explained by the poor quality of the structure, manifesting itself in a low $R_{\text {free }}$ value and high percentage of Ramachandran, sidechain, and real-space R-value Z-score (RSRZ) outliers (PDB: $2 \mathrm{HG}_{4}{ }^{[99]}$ ). Rosetta analysis of mFAS KS-AT chains without PAL were especially helpful, as we identified two residues interacting with the linker simply by comparing the predicted differences in energy with and without PAL (Tab. 3.6). Residue D61 was predicted to interact with PAL to a greater extent than 1457. And indeed, our experimental data supports this prediction. Overall, the importance of D61's interaction in the KS-AT can explain the stability problems in LD-AT swapping approaches in/with mFAS.

The tools with simpler algorithms than Rosetta (Robetta, Drug Score, mCSM, SpotOn) showed no correlation tendencies at all (Fig. S17). This discrepancy cannot be explained by program limitations to the usage of structures without PAL (Robetta, DrugScore, SpotOn) as the corresponding Rosetta predictions still showed clear correlation tendencies in mFAS KS-AT. Thus, our study indicates limitations of the programs and their algorithms themselves.

In conclusion, the simpler bioinformatics tools tested were not helpful for in silico analysis of the wild type. Rosetta, on the other hand, was shown to be useful for stability predictions of mutations under the premise that high quality structural information is available. Our protein engineering attempts have shown that the characterization of wild types may be key for successful strategies. Suitable in silico tools like Rosetta can save a lot of time by reliably predicting stability and robustness of the proteins involved. Furthermore, Rosetta's application in more complex in silico approaches on the chimeric level would be conceivable, since this method is not limited to alanine mutations. Proper modeling of chimeras might be combined with subsequent Rosetta calculations to estimate their stability. Promising candidates could then be experimentally analyzed regarding their stability and activity, finally paving the way to functional chimeras. 


\subsection{Conclusion and Outlook}

FASs and PKSs are evolutionary closely related enzymes. The AT domains of both systems are key players in their biosynthesis and responsible for loading acyl substrates onto the ACP domains of these multidomain complexes. The set of AT domains we focused on in this thesis comprises high transacylation rates of 15 molecules/s for iterative PKSs and up to several hundred molecules per second for type I and II FASs for both priming and elongation substrates. The tolerance and polyspecificity of ATs of iterative PKSs/FASs towards natural and non-natural substrates was already reported in previous studies. ${ }^{[9,230,241]}$ In contrast, the ATs of modular PKSs appear to have significantly decreased turnover rates (0.7-7 molecules/s) and substrate tolerance and to be adjusted to their function in selecting the specific elongation substrates for the synthesis of intricate secondary metabolites. Confirming the findings of previous studies, the specificity of AT domains analyzed in this study is in general not assured by hydrolytic proofreading. ${ }^{[133,143]}$

In addition to a basic characterization of AT kinetic properties, we showed that mutations at the interface between AT and ACP can lead to complex kinetic outputs, particularly when affecting the residues involved in the electrostatic network that guides and tunes enzyme-substrate interactions. We showed that replacing an arginine that is likely involved in a key electrostatic interaction at the AT:ACP interface by lysine, serine, or glutamate (R850K, R850S, R850E) leads to a severe drop in enzyme-substrate complex stability, catalytic efficiencies, and turnover rates. The impact of mutations can be explained by changing conformational properties that include those of active site and/or binding tunnel distant to the point mutation. The broadly changing kinetic parameters upon single mutations point towards a plasticity in the interaction of AT with acyl-CoA and ACP, as recently suggested for the AT-ACP interaction in the E. coli typell FAS (FabD and AcpP). [14]

Our findings on the kinetic properties of AT domains are highly relevant for AT-centered PKS engineering approaches. The present study can provide guidance in PKS engineering for different strategies to modify the product spectrum. In cases where AT domains are engineered for loading non-natural elongation substrates, the promiscuous ATs of iterative systems may be best suited. Particularly, the MAT domain of the mammalian FAS seems ideal for such approaches, as it appears in functional properties and phylogeny as evolutionary close to a FAS/PKS common ancestor. As a primordial-like enzyme with polyspecificity and rapid turnover rates, it can be regarded as better suited to acquire novel or altered functions by mutation than ATs from modular PKSs. ${ }^{238,242]}$ In other cases where AT domains are engineered to achieve the transacylation of a particular acyl compound of interest, two approaches are essentially possible: the introduction of specific mutations in the 


\section{Discussion}

AT binding site or the swap-in of an entire AT domain natively transferring the compound of interest. ${ }^{[75]}$ Both approaches have been performed successfully (for recent examples, see references $\left.{ }^{[20,243]}\right)$. While the first approach might be hampered by partially unspecific loading of substrates, the latter suffers from protein instability and loss of activity. We recommend harnessing the intrinsic specificities of AT domains from the pool of modular PKSs whenever possible, because those domains will likely provide the required selectivity.

Following this strategy, we tried to design a functional chimeric mFAS-based elongation module. Analysis of the wild type mFAS KS-AT revealed an outstandingly high robustness of the fold. Showing this characteristic of a primordial enzyme, mFAS was proven to be highly suited for domain swapping approaches. In our study, we identified one amino acid as essential binding hotspot in the KS-AT, which seems to be part of an electrostatic network stabilizing the fold. Based on this, we were able to identify proper domain boundaries in mFAS for an AT swap. This lays the foundation for the design of a stable and active chimeric elongation module.

In further experiments we tried to swap different substrate-specific ATs from other systems in mFAS to create stable and active chimeras. The ATs - even different ATs from the same PKS - behaved differently in these experiments using the same domain boundaries. In contrast to a previous study in which universally applicable domain boundaries for AT swapping in type I PKS modules were published, ${ }^{[20]}$ we found that every particular system behaves differently and small variations in domain boundaries can have a huge impact on protein stability and activity.

Many of our chimeric constructs showed poor stability. Eventually, we designed a stable chimera using DEBS3 AT6 which showed 70\% wild type donor AT activity. Unfortunately, the donor AT itself features slow transacylation rates and is of limited value for our application. As proof of principle, we implemented this chimera in a minimal elongation module, but received only low expression yields. Using the established domain boundaries, we were able to create another chimeric elongation module with EcPKS1 AT which featured high protein quality. Installing this stable chimeric elongation module in a minimal assembly line proved that priming by the elongation module was successfully suppressed. However, only minor product rates were detected.

Several parts of the assembly line might cause these limitations. The AT itself could feature slow transacylation rates, the artificial intramodular interactions in the chimeric elongation module might impede chain elongation, or the artificial intermodular interaction between priming and elongation module might hamper chain translocation. Identifying the root of the problem is key for optimizing the chimeric assembly line. Among other experiments, we will 


\section{Discussion}

have to take a step back and analyze the wild type AT. Analysis of the chimeric subconstruct KS-AT in the AT activity assay would provide information regarding the transacylation ability. The non-covalent intermodular linkers could be exchanged or replaced by a covalent linkage just like in modular PKSs. Overall, these findings strongly emphasize the value of detailed analysis and kinetic characterization of wild type donor ATs and chimeric subconstructs prior to installation in even a simple assembly line. For successful engineering approaches, detailed knowledge on the wild types should be considered in the design of chimeric assembly lines, ideally complemented by in silico approaches. Rosetta and the initial AT substrate screening could be used to estimate the robustness of the protein fold and the AT activity, respectively. This approach could enable selecting promising candidates for AT swapping in mFAS. 


\section{Experimental Procedures}

Parts of this chapter have been previously accepted for publication in: F. Stegemann, M. Grininger, "Transacylation Kinetics in Fatty Acid and Polyketide Synthases and its Sensitivity to Point Mutations", Chem CatChem (2021). [1] For individual contributions, copyright, and Creative Commons license, please see the statement of personal contributions.

\subsection{Material and Methods}

\subsubsection{Molecular Cloning}

Genes encoding for FAS and PKS constructs were cloned into pET-22b vector (Novagen), containing a pBR322 origin, ampicillin resistance, and lacl gene, which controls expression via the T7 polymerase system. Vectors encode an optional N-terminal Strep-tag and C-terminal His(6/8)-tag. Vectors were linearized by polymerase chain reaction (PCR) or enzymatic restriction. Phusion ${ }^{\circledR}$ High-Fidelity DNA Polymerase (NEB) and CloneAmp ${ }^{T M}$ $\mathrm{HiFi}$ PCR Premix (Clontech) were used to amplify genes of interest from template DNA by PCR with specific primers, generating complementary overhangs. Melting temperatures were calculated using oligocalc basic. ${ }^{[244]}$ Genes were inserted into the linearized vector via ligation independent cloning using the In-Fusion ${ }^{\circledR}$ HD Cloning Kit (Takara Bio). Multiple gene fragments were assembled by a MegaPrimer PCR to a single insert. For large PCR products ( $>5,000 \mathrm{bp}$ ), template DNA was digested with Dpnl (NEB). DNA was extracted from agarose gels using the NucleoSpin $3^{\circledR}$ Gel and PCR Clean-up (Marcherey-Nage) or the Wizard SV Gel and PCR Clean-Up System (Promega).

Cloning products were transformed in E.coli Stellar ${ }^{\mathrm{TM}}$ competent cells (Clontech) by heat shock. Cells were cultivated on liquid broth (LB)-agar (Lennox; Carl Roth) plates supplemented with $100 \mu \mathrm{g} / \mathrm{mL}$ ampicillin and $1 \%$ glucose at $37^{\circ} \mathrm{C}$ overnight or at room temperature over the weekend. Plates were stored in the dark up to several weeks at $4{ }^{\circ} \mathrm{C}$. Single colonies were used to inoculate $2-5 \mathrm{~mL}$ LB-media (Lennox; Carl-Roth) containing $100 \mu \mathrm{g} / \mathrm{mL}$ ampicillin and $1 \%$ glucose. Cells were cultivated at $37^{\circ} \mathrm{C}$ overnight or at $20^{\circ} \mathrm{C}$ over the weekend. Plasmids were isolated using the PureYield ${ }^{\text {TM }}$ Plasmid Miniprep System (Promega) and the GeneJETTM Plasmid Miniprep Kit (Thermo Fisher Scientific). Plasmids were controlled by Sanger sequencing (Microsynth Seqlab).

An excerpt of vectors used in this study is listed in the appendix. Plasmids pAR and pMJD were kindly provided by Dr. Alexander Rittner and Mirko Joppe. 


\subsubsection{Protein Expression}

Constructs were transformed in E. coli BL21-Gold (DE3) competent cells (Agilent Technologies) by heat shock. Constructs containing an ACP were co-transformed with a pETcoco vector (Novagen) encoding the 4'-PPT Sfp from B. subtilis or with a pCDF-1b vector (Novagen) encoding the 4'-PPT Npt from Streptomyces platensis for in vivo phosphopantetheinylation of the ACP. For mFAS ACP, a bicistronic vector encoding the ACP and Sfp from B. subtilis was used. Cells were cultivated on LB-agar (Lennox; Carl Roth) plates supplemented with $1 \%$ glucose and the respective selection antibiotica: $100 \mu \mathrm{g} / \mathrm{mL}$ ampicillin for single transformations, $50 \mu \mathrm{g} / \mathrm{mL}$ ampicillin and $17 \mu \mathrm{g} / \mathrm{mL}$ chloramphenicol for co-transformation with the pETcoco vector, and $50 \mu \mathrm{g} / \mathrm{mL}$ ampicillin and $25 \mu \mathrm{g} / \mathrm{mL}$ spectinomycin for co-transformations with the $\mathrm{pCDF}$ vector. Plates were incubated at $37^{\circ} \mathrm{C}$ overnight or at room temperature over the weekend and were stored at $4{ }^{\circ} \mathrm{C}$ up to several days, subsequently.

$20 \mathrm{~mL}$ LB-media (Lennox; Carl Roth) supplemented with the respective antibiotics and $1 \%$ glucose was inoculated with a randomly picked single clone. The pre-culture was incubated at $130-180 \mathrm{rpm}$ and at $37^{\circ} \mathrm{C}$ overnight or at $20^{\circ} \mathrm{C}$ over the weekend. $1 \mathrm{~L}$ terrific broth (TB)media was inoculated with the pre-culture and incubated at $37^{\circ} \mathrm{C}$ and $130-180 \mathrm{rpm}$ until an $\mathrm{OD}_{600}$ of 0.6-0.8 was reached. The culture was cooled down for $15 \mathrm{~min}$ at $4{ }^{\circ} \mathrm{C}$, prior to expression induction with $0.25 \mathrm{mM}$ isopropyl $\beta$-D-1-thiogalactopyranoside (IPTG). Expression was carried out at $20^{\circ}$ and $130-180 \mathrm{rpm}$ overnight. Cells were harvested by centrifugation at $4,000 \mathrm{rpm}$ for $20 \mathrm{~min}$. Cell pellets were immediately purified or stored at $-80^{\circ} \mathrm{C}$ for several days.

\subsubsection{Purification of AT Constructs and mFAS Modules}

Cell pellets were resuspended in corresponding $\mathrm{Ni}$ Buffer $\left(450 \mathrm{mM} \mathrm{NaCl}, 50 \mathrm{mM} \mathrm{NaH} \mathrm{PO}_{4}\right.$, $10 \mathrm{mM}$ imidazole, $20 \% \mathrm{v} / \mathrm{v}$ glycerol, $\mathrm{pH}$ 7.6) containing DNAse I and $1 \mathrm{mM}$ EDTA. Cells were mechanically disrupted at 1,000 bar using a French pressure cell press and centrifuged at $50,000 \mathrm{rcf}$ and $4{ }^{\circ} \mathrm{C}$ for $1 \mathrm{~h}$. After centrifugation, $2 \mathrm{mM} \mathrm{MgCl} 2$ were added. Constructs were

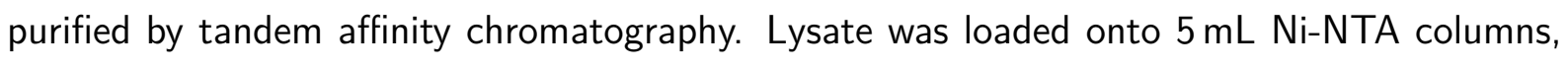
unbound protein was washed off with 5 column volumes (CV) Ni Buffer. Bound protein was eluted with 2.5 CV Ni Buffer containing $300 \mathrm{mM}$ imidazole. Subsequently, Ni elution fractions were loaded onto $5 \mathrm{~mL}$ Strep-Tactin columns (Iba), unbound protein was washed off with $6 \mathrm{CV}$ Strep Buffer ( $500 \mathrm{mM} \mathrm{NaCl}, 50 \mathrm{mM} \mathrm{NaH}_{2} \mathrm{PO}_{4}, 20 \% \mathrm{v} / \mathrm{v}$ glycerol, pH 7.6). Bound protein was eluted with $2.5 \mathrm{CV}$ Strep Buffer containing $2.5 \mathrm{mM}$ desthiobiotin. Elution fractions were analyzed by SDS-PAGE. Proteins were concentrated using Amicon Ultra Centrifugal Filters (Merck Millipore), flash frozen in liquid nitrogen, and stored in aliquots at $-80^{\circ} \mathrm{C}$. 


\section{Experimental Procedures}

\subsubsection{Purification of $\mathrm{ACPS}$}

Cell pellets were resuspended in corresponding $\mathrm{Ni}$ Buffer $\left(200 \mathrm{mM} \mathrm{NaCl}, 50 \mathrm{mM} \mathrm{NaH} \mathrm{PO}_{4}\right.$, $20 \mathrm{mM}$ imidazole, $10 \% \mathrm{v} / \mathrm{v}$ glycerol, $\mathrm{pH} 7.4$ ) containing DNAse I and $1 \mathrm{mM}$ EDTA. Cells were mechanically disrupted at 1,000 bar using a French pressure cell press and centrifuged at $50,000 \mathrm{rcf}$ and $4{ }^{\circ} \mathrm{C}$ for $1 \mathrm{~h}$. After centrifugation, $2 \mathrm{mM} \mathrm{MgCl} 2$ were added. E. coli ACP was activated in vitro with Sfp prior to purification. All ACPs were purified by Ni-affinity chromatography. Lysate was loaded onto $5 \mathrm{~mL}$ Ni-NTA columns, unbound protein was washed off with $5 \mathrm{CV}$ Ni Buffer. Bound protein was eluted with 2.5 CV Ni Buffer containing $300 \mathrm{mM}$ imidazole. ACPs were further purified by SEC using HiLoad 16/600 Superdex 200 pg column (200 mM NaCl, $50 \mathrm{mM} \mathrm{NaH} \mathrm{PO}_{4}, 10 \%$ v/v glycerol, pH 7.4, filtered and degased). The column was calibrated with the Gel Filtration LMW and HMW Calibration Kits (GE Healthcare). Elution fractions were analyzed by SDS-PAGE. ACP was pooled after SEC to create one stock of each ACP as substrate used for all measurements of the AT activity assay. This should minimize errors in assay resulting from inconsistent protein expression/quality. Proteins were concentrated using Amicon Ultra Centrifugal Filters (Merck Millipore), flash frozen in liquid nitrogen, and stored in aliquots at $-80^{\circ} \mathrm{C}$. Complete kinetic characterization of AT domains requires high yield and high quality of the corresponding ACPs. Expression resulted in insufficient yields of most ACPs. Using codon-optimized sequences for expression in E. coli, the holo-ACP yields increased significantly.

\subsubsection{Protein Concentration}

Protein concentration was determined from the absorbance at $280 \mathrm{~nm}$ using a NanoDrop 2000c (Thermo Fisher Scientific). Extinction coefficient and absorbance of $1 \mathrm{~g} / \mathrm{L}$ at $280 \mathrm{~nm}$ $(10 \mathrm{~mm})$ were calculated from the primary sequence with CLC Main Workbench (QIAGEN Bioinformatics). Values are given in tables 5.1 to 5.5 for E.coli ACP with, for all other proteins without the $\mathrm{N}$-terminal methionine. 
Table 5.1: Absorbance of $1 \mathrm{~g} / \mathrm{L}$ wild type proteins at $280 \mathrm{~nm}(10 \mathrm{~mm})$.

\begin{tabular}{ccc}
\hline Protein & Species & Abs. \\
\hline \multirow{7}{*}{ ACP } & mouse & 0.475 \\
& E. coli & 0.131 \\
& AVES1M0 & 0.650 \\
& DEBS1M0 & 1.367 \\
& DEBS3M5 & 0.842 \\
& DEBS3M6 & 0.469 \\
& PikAllIM5 & 0.857 \\
& RAPS3M14 & 0.917 \\
& Pks7 & 1.500 \\
\hline & E. coli & 1.171 \\
& AVES1M0 & 1.187 \\
& DEBS1M0 & 1.456 \\
KS(0)_)AT & DEBS3M5 & 1.021 \\
& DEBS3M6 & 0.994 \\
& PikAllIM5 & 1.124 \\
& RAPS3M14 & 1.208 \\
& Pks7 & 1.266 \\
\hline
\end{tabular}

Table 5.2: Absorbance of $1 \mathrm{~g} / \mathrm{L}$ DEBS3M5 AT interface mutants at $280 \mathrm{~nm}(10 \mathrm{~mm})$.

\begin{tabular}{ccc} 
Construct & Mutation & Abs. \\
\hline \multirow{4}{*}{$\mathrm{KS}^{0}$-AT } & A539S & 1.021 \\
& A539D & 1.021 \\
& A539E & 1.021 \\
& R539F & 1.021 \\
& R850K & 1.022 \\
& R850E & 1.022 \\
& R850F & 1.022 \\
& R850S & 1.022 \\
\hline
\end{tabular}




\section{Experimental Procedures}

Table 5.3: Absorbance of $1 \mathrm{~g} / \mathrm{L} \mathrm{mFAS} \mathrm{KS-AT} \mathrm{mutants} \mathrm{at} 280 \mathrm{~nm}(10 \mathrm{~mm})$.

\begin{tabular}{ccc}
\hline Construct & Mutation & Abs. \\
\hline & D61A & 1.051 \\
N96A & 1.051 \\
& R429A & 1.052 \\
& I457A & 1.051 \\
& R468A & 1.052 \\
mFAS (KS $\left.{ }^{0}-\mathrm{AT}\right)$ & Y470A & 1.036 \\
& H425A & 1.052 \\
& V479A & 1.051 \\
& E481A & 1.051 \\
& Q483A & 1.051 \\
& H804A & 1.051 \\
& L805A & 1.051 \\
\hline
\end{tabular}

Table 5.4: Absorbance of $1 \mathrm{~g} / \mathrm{L}$ DEBS3M5 KS-AT mutants at $280 \mathrm{~nm}(10 \mathrm{~mm})$.

\begin{tabular}{ccc}
\hline Construct & Mutation & Abs. \\
\hline & E129A & 1.022 \\
& D134A & 1.021 \\
DEBS3M5 (KS $\left.{ }^{0}-A T\right)$ & R510A & 1.022 \\
& V518A & 1.021 \\
& Y867A & 1.009 \\
& A868R & 1.020 \\
\hline
\end{tabular}


Table 5.5: Absorbance of $1 \mathrm{~g} / \mathrm{L}$ chimeric proteins at $280 \mathrm{~nm}(10 \mathrm{~mm})$.

\begin{tabular}{cccc}
\hline Construct & Protein number & Swapped domain & Abs. \\
\hline & FS37 & DEBS3AT5 & 0.900 \\
& FS38 & DEBS3(LD-AT)5 & 0.993 \\
& FS57 & DEBS3(AT)5 & 0.960 \\
& FS58 & DEBS3(AT)5 & 0.899 \\
& FS59 & DEBS3(AT)5 & 0.959 \\
KS-AT & FS60 & DEBS3(LD-AT)5 & 0.990 \\
& FS61 & DEBS3(LD-AT)5 & 0.992 \\
& FS62 & DEBS3(LD-AT)5 & 0.989 \\
& FS116 & FabD & 0.942 \\
& FS117 & FabD & 0.939 \\
& FS118 & DEBS3(LD-AT)6 & 0.894 \\
KS-AT-ACP & FS119 & DEBS3(LD-AT)6 & 0.895 \\
\hline \multirow{6}{*}{ FS172 } & EcPKS1(LD-AT-ACP) & 0.963 \\
\hline \multirow{6}{*}{ FAS } & FS31 & DEBS3(AT)5 & 0.840 \\
& FS32 & DEBS3(LD-AT)5 & 0.871 \\
& FS33 & DEBS3(LD-AT)5 & 0.873 \\
& FS34 & RAPS3(AT)14 & 0.838 \\
& FS35 & RAPS3(LD-AT)14 & 0.919 \\
& FS36 & RAPS3(LD-AT)14 & 0.921 \\
\hline & & & \\
& & &
\end{tabular}

\subsubsection{SDS-PAGE Analysis}

Proteins were analyzed in their quality by SDS-PAGE. After incubation with Laemmli buffer at $95^{\circ} \mathrm{C}$ for $5 \mathrm{~min}$, a $10 \mu \mathrm{L}$ sample of about $0.05 \mathrm{mg} / \mathrm{mL}$ protein dissolved in water was loaded. Self-cast $10 \%$ (for $30-300 \mathrm{kDa}$ proteins), $15 \%$ (for $10-30 \mathrm{kDa}$ proteins), and NuPAGETM 4-12\% Bis-Tris Protein Gels (Invitrogen) were used. Gel electrophoresis was performed in Tris/Glyine/SDS running buffer (Bio-Rad) or NuPAGETM MES SDS running buffer (Invitrogen) at $200 \mathrm{~V}$ for $45-60 \mathrm{~min}$. InstantBlue ${ }^{\mathrm{TM}}$ Coomassie Protein Stain (Expedeon) was used for staining. 


\section{Experimental Procedures}

\subsubsection{Mass Spectrometric Analysis of ACPs}

Full phosphopantetheinylation of ACPs was controlled by MS. Proteins were precipitated and resolved in sterile water. An ultimate 3000 RSLC system (Dionex) coupled to an impact II (Bruker, for non-codon optimized ACPs) or to a micrOTOF-Q II (Bruker, for codon optimized ACPs) equipped with an electrospray ionization source was used to perform HPLC-MS of ACPs. For chromatographic separation an RP-C3 column (Zorbax 300-SB, $300 \AA$, $150 \mathrm{~mm} \times$ $3.0 \mathrm{~mm} \times 3.5 \mu \mathrm{m}$, Agilent) was used with a mobile phase system consisting of solvent $A$ (water with $0.1 \%(\mathrm{v} / \mathrm{v})$ formic acid) and solvent $B$ (acetonitrile with $0.1 \%(\mathrm{v} / \mathrm{v})$ formic acid). After equilibration with $15 \%$ solvent $B$ and a flow of $0.6 \mathrm{~mL} / \mathrm{min}$ for $1.5 \mathrm{~min}$, the concentration of solvent $B$ was linearly increased to $65 \%$ over $22.5 \mathrm{~min}$. This was followed by a linear increase of solvent $B$ to $95 \%$ over 4 min prior to re-equilibration of $15 \%$ solvent $B$ for $2 \mathrm{~min}$. Proteins were detected by absorbance at $280 \mathrm{~nm}$. ACPs were found to elute at $3.5-16 \mathrm{~min}$. MS data was acquired in positive mode in the range of $50-2000 \mathrm{~m} / \mathrm{z}$ and analyzed using DataAnalysis 4.3 software (Bruker Dalton $\mathrm{GmbH}$ ).

\subsubsection{CoA-488 Assay}

The CoA-488 assay was used as fast in-house method for analysis of ACP activation. ACP was incubated with 0.5 equivalents purified Sfp, 5 equivalents CoA-488 (NEB), and 5,000 equivalents $\mathrm{MgCl}_{2}$ protected from light at $37^{\circ} \mathrm{C}$ and $350 \mathrm{rpm}$ for $1 \mathrm{~h}$. Laemmli buffer was added and the sample was incubated at $95^{\circ} \mathrm{C}$ for $5 \mathrm{~min} .10 \mu \mathrm{L}$ sample was loaded onto a NuPAGETM Bis-Tris 4-12\% Protein Gel (Invitrogen). Electrophoresis was carried out in NuPAGETM MES SDS running buffer (Invitrogen) at $200 \mathrm{~V}$ for $1 \mathrm{~h}$. In-gel fluorecence was detected at the Fusion SL Fluorescence Imaging System (VilberLourmat) at $488 \mathrm{~nm}$, prior to gel staining with InstantBlue ${ }^{T M}$ Coomassie Protein Stain (Expedeon).

\subsubsection{HPLC-SEC Analysis}

Quality and oligomeric state of all AT wild types and chimeric proteins were analyzed by HPLCSEC ( $500 \mathrm{mM} \mathrm{NaCl}, 50 \mathrm{mM} \mathrm{NaH}_{2} \mathrm{PO}_{4}, 5 \% \mathrm{v} / \mathrm{v}$ glycerol, $\left.\mathrm{pH} 7.6\right)$ at room temperature with a flow of $0.3 \mathrm{ml} / \mathrm{min}$. An UltiMate 3000 RSLC (Dionex) equipped with an UV-vis array detector was used with SEC columns bioZen $1.8 \mu \mathrm{m}$ SEC-3, Yarra-SEC-X150, and Yarra-SEC-X300. Proteins were detected by absorbance at $280 \mathrm{~nm}$. Buffers and samples were filtered through $0.1 \mu \mathrm{m}$. $8 \mu \mathrm{L}$ protein sample was injected. Columns were calibrated with the Gel Filtration LMW and HMW Calibration Kits (GE Healthcare). 


\subsubsection{Thermal Shift Assay}

TSA was performed to analyze all constructs in their stability using a CFX96 Touch Real-Time PCR Detection System (BioRad) with excitation and emission wavelength set to 450-490 and $560-580 \mathrm{~nm}$, respectively. Fluorescence of the dye SYPRO Orange was measured from 5 to $95^{\circ} \mathrm{C}$ with $0.5^{\circ} \mathrm{C}$ steps per minute in white Multiplate ${ }^{\mathrm{TM}}$ 96-well PCR Plates (BioRad). Melting curves of all wild type AT constructs and ACPs were measured in storage buffer and AT activity assay buffer. DEBS3M5 AT:ACP mutants were measured in storage buffer. mFAS and DEBS3M5 KS-AT wild types and mutants were measured in four different buffers. Chimeric constructs were analyzed in four different buffers or in storage buffer. Analysis was performed using OriginPro 9.1.

\subsubsection{AT Activity Assay}

The AT activity assay was adapted from references. ${ }^{[9,133,211]}$ It couples formation of free $\mathrm{CoA}$ with the reduction of nicotinamide adenine dinucleotide $\left(\mathrm{NAD}^{+}\right)$, which can then be followed fluorimetrically, allowing the quantification of transferase activity. The acyl transfer is measured in presence and absence of ACP, giving access to transacylation and hydrolysis rates, respectively. Kinetic hydrolysis parameters are calculated by titration of X-CoA. To determine absolute kinetic transacylation parameters, $\mathrm{X}-\mathrm{CoA}$ is titrated to a series of fixed ACP concentrations.

The AT activity assay buffer $\left(50 \mathrm{mM} \mathrm{NaH} \mathrm{PO}_{4}, 1 \mathrm{mM}\right.$ EDTA, $1 \mathrm{mM}$ DTT added directly before usage, $10 \% \mathrm{v} / \mathrm{v}$ glycerol, $\mathrm{pH} 7.6$ ) was used to set up all other solutions for this assay. For all proteins and substrates, fresh aliquots were used. For each system, the same batch of substrates (ACP and acyl-CoAs) was used. Four master mixes in $4 \times$ concentration were prepared. For the acyltransferase solutions the proteins were diluted to $0.1-400 \mathrm{nM}$ final assay concentration with assay buffer supplemented with $0.1 \mathrm{mg} / \mathrm{mL}$ BSA. The ACP solution was prepared by diluting highly pure and concentrated ACP to $2.5-530 \mu \mathrm{M}$ final concentration with the assay buffer. Substrate acyl-CoA solution was also prepared with the assay buffer in different concentrations ranging from $1 \mu \mathrm{M}$ to $1 \mathrm{mM}$ final concentration. The read out solution contained $2 \mathrm{mM} \alpha$-ketoglutaric acid, $0.4 \mathrm{mM} \mathrm{NAD}^{+}, 0.4 \mathrm{mM}$ TPP and 5 to $15 \mathrm{mU} / 100 \mu \mathrm{L} \alpha \mathrm{KGDH}$ as final concentrations. All solutions were pre-heated/incubated at $25^{\circ} \mathrm{C}$ for at least $5 \mathrm{~min}$. Assays were performed in 96-well f-bottom microtiter plates (Greiner Bio-one). For transacylation, $25 \mu \mathrm{L}$ of each solution was added, ACP was added via the dispenser to initiate the reaction. For hydrolysis, assay buffer was used to compensate the volume of ACP. For background measurements the AT solution was replaced by $0.1 \mathrm{mg} / \mathrm{mL}$ BSA in assay buffer. NADH fluorescence was monitored for $5 \mathrm{~min}$ at $25^{\circ} \mathrm{C}$, taking equidistant measurements every $20 \mathrm{~s}$ using a ClarioStar microplate reader (BMG labtech). The following 


\section{Experimental Procedures}

settings were used: excitation: $348-20 \mathrm{~nm}$, emission: $476-20 \mathrm{~nm}$, gain: 1900 , focal height: $5.2 \mathrm{~mm}$, flashes: 70 , orbital averaging: $4 \mathrm{~mm}$. For kinetic characterization of the chimeras FS118 and FS139 as well as the wild type FS139, the assay was performed in 384 Well Small Volume ${ }^{\mathrm{TM}}$ HiBase Microplates (Greiner Bio-One) with a total volume of $20 \mu \mathrm{L}$ and the following settings: excitation: $348-20 \mathrm{~nm}$, emission: $476-20 \mathrm{~nm}$, gain: 1500 , focal height: $5.2 \mathrm{~mm}$, flashes: 17 .

To meet Michaelis-Menten requirements, the substrate concentration was always kept significantly higher than the enzyme concentration, at least ten times. ${ }^{[129]}$ For hydrolysis and for each ACP curve in transacylation measurements, initial experiments were performed to estimate the (apparent) $\mathrm{K}_{\mathrm{m}}^{\mathrm{X}-\mathrm{CoA}}$. Based on these, the acyl-CoA substrate concentration was varied to calculate kinetic parameters: $(0.2 ; 0.3 ; 0.5 ; 0.75 ; 1.25 ; 2 ; 3 ; 5) \times \mathrm{K}_{\mathrm{m}}^{\mathrm{X}-\mathrm{CoA}}$, app. In order to ensure that the measurement is performed in the initial quasi-linear range of substrate consumption, the product concentration has to be significantly lower than the substrate concentration. [129] The increase during measurement was always linear and substrate consumption was ensured to be below $10 \%$ for the majority of assays performed. Some measurements showed significantly higher substrate consumption. In such cases, those measurements were inlcuded, as long as the increase was shown to be linear and the Michaelis-Menten fit as well as the kinetic parameters do not change considerably. If not mentioned otherwise, transacylation was performed in biological triplicates. Hydrolysis was performed in technical triplicates of biological triplicates or technical/biological triplicates. Background values were subtracted from the average of technical/biological measurement values. Occasional dust or air bubbles caused strong outliers. These were omitted for further analysis after careful analysis. The time span of 40-120 and 20-120 s was analyzed for transacylation and hydrolysis, respectively. For high substrate consumption, this time span was adapted. In order to convert relative fluoresence units (RFU) into concentration, an NADH calibration curve was measured (Fig. S23). Hydrolysis kinetic parameters were calculated by Michaelis-Menten plots using OriginPro 9.1. Transacylation parameters were calculated by global Michaelis-Menten fits using OriginPro 9.1.

\subsubsection{TAL Assay}

For quantification of TAL derivative production, the TAL assay was performed in glass cuvettes Suprasil type no. 117.104-QS $10 \mathrm{~mm}$ (Hellma) at NanoDrop 2000c (Thermo Fisher Scientific) in cuvette mode, following the absorbance at $298 \mathrm{~nm}$ and $25^{\circ} \mathrm{C}$. Priming and elongation module as well as priming (Prop or Ac) and elongation substrate (MMal) were pipetted together in assay buffer ( $400 \mathrm{mM} \mathrm{NaPi}, 4 \%$ glycerol, $5 \mathrm{mM}$ TCEP, pH 7.2). Reaction was started with elongation substrate. Final concentrations were 0 or $100 \mu \mathrm{M}$ substrates, $0-4 \mu \mathrm{M}$ priming module, 1-3 $\mu \mathrm{M}$ elongation module. The molar extinction coefficient at $298 \mathrm{~nm} \epsilon=2540 \mathrm{M}^{-1} \mathrm{~cm}^{-1}$ for TAL was used for conversion of absorbance into concentration. [245] 


\subsubsection{Design of DEBS3M5 AT:ACP |nterface Mutations}

Since the AT:ACP interface of DEBS3M5 and the structure of its ACP is not known, SwissModel was used to model the ACP domain. [246] The sequence was obtained from the Uniprot database (Q03133, residues 1392-1467). As structural template served the ACP from type I modular PKS from Mycobacterium ulcerans solved by nuclear magnetic resonance (NMR) spectroscopy (PDB: $5 \mathrm{HVC}$, identity percentage: $47 \%$, similarity: 0.40 ). Global model quality estimation (GMQE) by SwissModel was 0.73 , and the quality model energy analysis (QMEAN) was -1.93. Subsequently, three AT:ACP interfaces were used as template for structural alignment of the modeled $\mathrm{ACP}$ and the structure of AT domain (PDB: $2 \mathrm{HG} 4{ }^{[99]}$ ). The in silico docking study on human FAS as well as crystal structures of the AT:ACP complex of vicenistatin PKS VinK:VinL and of disorazole synthase (DSZS) served as templates. $[125,126,159]$ Based on these three alignments, PISA was used to identify hotspots in the AT:ACP interface. ${ }^{[247]}$ All three models gave the amino acids A539 and R850 as possible interfacial hotspots.

\subsubsection{Bioinformatics Tools}

Different bioinformatics tools were used to predict hotspots in the mFAS and DEBS3M5 wild type KS:LD and LD:AT interfaces, namely Prodigy, ${ }^{248]}$ Rosetta, ${ }^{249]}$ Robetta, [250] DrugScore, ${ }^{[251]} \mathrm{mCSM},{ }^{[252]}$ and SpotOn. ${ }^{253]}$ These programs use different algorithms to predict interactions between chains or effects of mutations on protein stability based on structural information (PDBs: 5MY0 and 2HG4). For this purpose, some programs require splitting of crystal structures into chains for the three domains KS, LD, and AT. For these programs the PAL (Prodigy, Robetta, DrugScore, SpotOn) was neglected, which potentially can influence the predictions, since interactions with the PAL are not considered.

The program Prodigy was used to identify interactions within the two interfaces. This program gives a list of interacting residues of two separate chains with the classification in weak and strong interactions. This list of predicted interactions was analyzed in the crystal structure. Potential interesting residues were further analyzed with the other bioinformatics tools. 


\section{Experimental Procedures}

The program mCSM performs a random mutation scan on a single residue, resulting in a list of all possible amino acid mutations with predicted changes in Gibbs free energy $\Delta \Delta \mathrm{G}=\Delta \mathrm{G}(\mathrm{WT})-\Delta \mathrm{G}(\mathrm{Mut})$ in $\mathrm{kcal} / \mathrm{mol}$, with the Gibbs free energy of wild type (WT) and mutant (Mut). For destabilizing effects of the mutation introduced, the predicted $\Delta \Delta \mathrm{G}$ is negative, for stabilizing effects, the predicted $\Delta \Delta \mathrm{G}$ is positive. All other programs define the change in Gibbs free energy as $\Delta \Delta \mathrm{G}=\Delta \mathrm{G}$ (Mut) $-\Delta \mathrm{G}$ (WT). For better comparability, the sign was changed for mCSM predictions. The higher (positive) the change in Gibbs free energy, the more destabilizing a mutation.

The program Rosetta performs a random point mutation scan on all residues. Similar to mCSM the output is a list of all possible amino acid mutations with predicted changes in Gibbs free energy in REU, which is a generic energy unit.

The programs Robetta and DrugScore are limited to an alanine scan. Predictions are made for alanine mutation of all residues. Both tools give a list of mutations with specific predicted changes in Gibbs free energy in $\mathrm{kcal} / \mathrm{mol}$. The program DrugScore provides additional information on degree of buriedness and identifies possible saltbridges.

The program SpotOn is analyzing two chains in their interfacial contacts, classifying them in so-called Nullspots and Hotspots. Based on alanine mutations, the change in Gibbs free energy is predicted. Nullspots are defined as mutations with predicted changes $<2 \mathrm{kcal} / \mathrm{mol}$, hotspots as mutations with predicted changes $\geq 2 \mathrm{kcal} / \mathrm{mol}$. A list of predicted Nullspots and Hotspots is provided without specific $\Delta \Delta \mathrm{G}$ values.

Informations of all programs were compared. Those mutations were introduced, which were most likely to have a huge impact on proteins stability according to the different tools. 


\subsection{Synthesis of 2-Methylbutyryl-CoA}<smiles>CCC(C)C(=O)O</smiles>
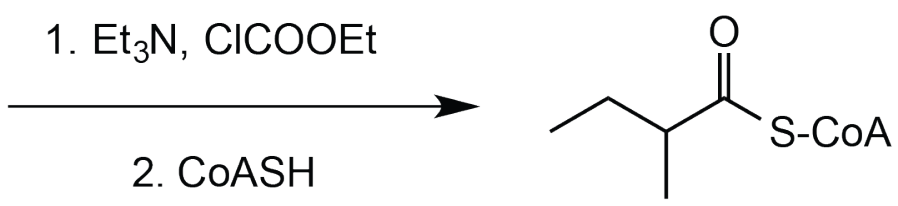

2-Methylbutyric acid $(34.5 \mathrm{mg}, 98 \%, 0.33 \mathrm{mmol}, 6.6$ equiv.) was dissolved in THF $(2 \mathrm{~mL})$ and cooled to $0{ }^{\circ} \mathrm{C}$. $\mathrm{Et}_{3} \mathrm{~N}(40 \mu \mathrm{L})$ and ethyl chloroformate $(27 \mu \mathrm{L}, 0.28 \mathrm{mmol}, 5.6$ equiv. $)$ were added and the solution was stirred for 45 minutes at $0{ }^{\circ} \mathrm{C}$. The reaction was centrifuged for 4 minutes at $5,000 \times \mathrm{g}$ for removal of insoluble salts. $1.5 \mathrm{~mL}$ of supernatant were added to $\mathrm{CoA} \mathrm{Li} 3\left(40.1 \mathrm{mg}, 0.051 \mathrm{mmol}, 1.0\right.$ equiv.) and $\mathrm{NaHCO}_{3}(32.1 \mathrm{mg}, 0.38 \mathrm{mmol}$, 7.6 equiv.) dissolved in water $(2 \mathrm{~mL})$. After stirring for 1 hour at room temperature, the solvents were removed using a SpeedVac. The raw product was dissolved in water $(2 \mathrm{~mL})$ and purified by preparative HPLC. Solvents were removed by lyophilization overnight, yielding 2-methylbutyryl-CoA $(38.0 \mathrm{mg}, 0.045 \mathrm{mmol}, 88 \%)$ as white powder. Synthesis adopted from references. ${ }^{[254,255]}$

${ }^{1} \mathrm{H}-\mathrm{NMR}\left(500 \mathrm{MHz}, \mathrm{MeOH}-\mathrm{d}_{4}\right) \delta(\mathrm{ppm})=8.63(\mathrm{~s}, 1 \mathrm{H}), 8.42(\mathrm{~s}, 1 \mathrm{H}), 6.20\left(\mathrm{~d},{ }^{3} \mathrm{~J}=5.00 \mathrm{~Hz}\right.$, $1 \mathrm{H})$, 4.93-4.85 (m, 2H), 4.60-4.59 (m, $1 \mathrm{H}), 4.33-4.23(\mathrm{~m}, 2 \mathrm{H}), 4.01(\mathrm{~s}, 1 \mathrm{H}), 3.91-3.85(\mathrm{~m}$, $1 \mathrm{H}), 3.66-3.61(\mathrm{~m}, 1 \mathrm{H}), 3.44\left(\mathrm{t},{ }^{3} \mathrm{~J}=5.00 \mathrm{~Hz}, 2 \mathrm{H}\right), 3.32\left(\mathrm{t},{ }^{3} \mathrm{~J}=5.00 \mathrm{~Hz}, 2 \mathrm{H}\right), 2.98\left(\mathrm{t},{ }^{3} \mathrm{~J}\right.$ $=5.00 \mathrm{~Hz}, 2 \mathrm{H}), 2.68-2.61(\mathrm{~m}, 1 \mathrm{H}), 2.42(\mathrm{t}, 3 \mathrm{~J}=5.00 \mathrm{~Hz}, 2 \mathrm{H}), 1.64-1.55(\mathrm{~m}, 1 \mathrm{H}), 1.48-1.38$ $(\mathrm{m}, 1 \mathrm{H}), 1.11-1.08(\mathrm{~m}, 3 \mathrm{H}), 0.95-0.93(\mathrm{~m}, 3 \mathrm{H}), 0.84-0.81(\mathrm{~m}, 6 \mathrm{H})$.

MALDI-MS (m/z): $[\mathrm{M}+\mathrm{H}]^{+}$calc. for $\mathrm{C}_{26} \mathrm{H}_{45} \mathrm{~N}_{7} \mathrm{O}_{17} \mathrm{P}_{3} \mathrm{~S}$ : 852.18000, found: 852.18009. 


\section{References}

[1] Stegemann, F. \& Grininger, M. Transacylation Kinetics in Fatty Acid and Polyketide Synthases and its Sensitivity to Point Mutations. ChemCatChem (2021, accepted article). URL https://doi.org/10.1002/cctc. 202002077.

[2] Nussbaum, F. V., Brands, M., Hinzen, B., Weigand, S. \& Dieter, H. Antibacterial Natural Products in Medicinal Chemistry - Exodus or Revival? Angewandte Chemie International Edition 45, 5072-5129 (2006).

[3] Smith, S. \& Tsai, S.-C. The Type I Fatty Acid and Polyketide Synthases: A Tale of Two Megasynthases. Natural Product Reports 24, 1041-1072 (2007).

[4] Staunton, J. \& Weissman, K. J. Polyketide biosynthesis: A millennium review. Natural Product Reports 18, 380-416 (2001).

[5] Hertweck, C. The biosynthetic logic of polyketide diversity. Angewandte Chemie International Edition 48, 4688-4716 (2009).

[6] Nivina, A., Yuet, K. P., Hsu, J. \& Khosla, C. Evolution and Diversity of Assembly-Line Polyketide Synthases. Chemical Reviews 119, 12524-12547 (2019).

[7] Maier, T., Leibundgut, M., Boehringer, D. \& Ban, N. Structure and function of eukaryotic fatty acid synthases. Quarterly Reviews of Biophysics 43, 373-422 (2010).

[8] Brignole, E. J., Smith, S. \& Asturias, F. J. Conformational flexibility of metazoan fatty acid synthase enables catalysis. Nature Structural and Molecular Biology 16, 190-197 (2009).

[9] Rittner, A., Paithankar, K. S., Huu, K. V. \& Grininger, M. Characterization of the Polyspecific Transferase of Murine Type I Fatty Acid Synthase (FAS) and Implications for Polyketide Synthase (PKS) Engineering. ACS Chemical Biology 13, 723-732 (2018).

[10] Schweizer, E. Microbial Type I Fatty Acid Synthases (FAS): Major Players in a Network of Cellular FAS Systems. Microbiology and Molecular Biology Reviews 68, 501-517 (2004).

[11] Tehlivets, O., Scheuringer, K. \& Kohlwein, S. D. Fatty acid synthesis and elongation in yeast. Biochimica et Biophysica Acta - Molecular and Cell Biology of Lipids 1771, 255-270 (2007).

[12] Erdbrügger, P. \& Fröhlich, F. The role of very long chain fatty acids in yeast physiology and human diseases. Biological Chemistry 402, 25-38 (2020). 
[13] Fersht, A. Structure and mechanism in protein science. A guide to enzyme catalysis and protein (W. H. Freeman and Company, New York, 1999), 2nd print edn.

[14] Misson, L. E. et al. Interfacial plasticity facilitates high reaction rate of E. coli FAS malonyl-CoA:ACP transacylase, FabD. Proceedings of the National Academy of Sciences of the United States of America 117, 24224-24233 (2020).

[15] Chang, S. I. \& Hammes, G. G. Structure and Mechanism of Action of a Multifunctional Enzyme: Fatty Acid Synthase. Accounts of Chemical Research 23, 363-369 (1990).

[16] Pieper, R., Ebert-Khosla, S., Cane, D. \& Khosla, C. Erythromycin biosynthesis: Kinetic studies on a fully active modular polyketide synthase using natural and unnatural substrates. Biochemistry 35, 2054-2060 (1996).

[17] Rittner, A., Paithankar, K. S., Drexler, D. J., Himmler, A. \& Grininger, M. Probing the modularity of megasynthases by rational engineering of a fatty acid synthase Type I. Protein Science 28, 414-428 (2019).

[18] Weissman, K. J. \& Leadlay, P. F. Combinatorial biosynthesis of polyketides. Nature Reviews Microbiology 3, 925-936 (2005).

[19] Rangan, V. S. \& Smith, S. Alteration of the substrate specificity of the malonylCoA/acetyl- CoA:acyl carrier protein S-acyltransferase domain of the multifunctional fatty acid synthase by mutation of a single arginine residue. Journal of Biological Chemistry 272, 11975-11978 (1997).

[20] Yuzawa, S. et al. Comprehensive in vitro analysis of acyltransferase domain exchanges in modular polyketide synthases and its application for short-chain ketone production. ACS Synthetic Biology 6, 139-147 (2017).

[21] Newman, D. J. \& Cragg, G. M. Natural Products as Sources of New Drugs from 1981 to 2014. Journal of Natural Products 79, 629-661 (2016).

[22] Wani, M. C., Taylor, H. L., Wall, M. E., Coggon, P. \& McPhail, A. T. Plant antitumor agents. VI. Isolation and structure of taxol, a novel antileukemic and antitumor agent from Taxus brevifolia. J. Am. Chem. Soc. 93, 2325-2327 (1971).

[23] Schwecke, T. et al. The biosynthetic gene cluster for the polyketide immunosuppressant rapamycin. Proceedings of the National Academy of Sciences of the United States of America 92, 7839-7843 (1995).

[24] Ansdell, V. E., Boosey, C. M., Geddes, A. M. \& Morgan, H. V. Malaria in Birmingham 1968-73. British Medical Journal 2, 206-208 (1974). 
[25] Wright, G. D. Unlocking the potential of natural products in drug discovery. Microbial Biotechnology 12, 55-57 (2019).

[26] Cortes, J., Haydock, S. F., Roberts, G. A., Bevitt, D. J. \& Leadlay, P. F. An unusually large multifunctional polypeptide in the erythromycin-producing polyketide synthase of Saccharopolyspora erythraea. Nature 348, 176-178 (1990).

[27] Brockmann, H. \& Henkel, W. Pikromycin, ein bitter schmeckendes Antibioticum aus Actinomyceten. Chemische Berichte 84, 284-288 (1951).

[28] Margalith, P. \& Pagani, H. Rifomycin. XIV. Production of rifomycin B. Applied Microbiology 9, 325-334 (1961).

[29] Civjan, N. (ed.) Natural Products in Chemical Biology (Wiley, Hoboken, NJ, 2012).

[30] Spellberg, B. et al. The epidemic of antibiotic-resistant infections: A call to action for the medical community from the infectious diseases society of America. Clinical Infectious Diseases 46, 155-164 (2008).

[31] Peregrín-Alvarez, J. M., Tsoka, S. \& Ouzounis, C. A. The phylogenetic extent of metabolic enzymes and pathways. Genome Research 13, 422-427 (2003).

[32] Peregrín-Alvarez, J. M., Sanford, C. \& Parkinson, J. The conservation and evolutionary modularity of metabolism. Genome Biology 10: R63 (2009).

[33] Dias, D. A., Urban, S. \& Roessner, U. A Historical overview of natural products in drug discovery. Metabolites 2, 303-336 (2012).

[34] Smith, S. The animal fatty acid synthase: one gene, one polypeptide, seven enzymes. The FASEB Journal 8, 1248-1259 (1994).

[35] Ibarguren, M., López, D. J. \& Escribá, P. V. The effect of natural and synthetic fatty acids on membrane structure, microdomain organization, cellular functions and human health. Biochimica et Biophysica Acta (BBA) - Biomembranes 1838, 1518 - 1528 (2014).

[36] Dibrova, D. V., Galperin, M. Y. \& Mulkidjanian, A. Y. Phylogenomic reconstruction of archaeal fatty acid metabolism. Environmental Microbiology 16, 907-918 (2014).

[37] Beld, J., Lee, D. J. \& Burkart, M. D. Fatty acid biosynthesis revisited: structure elucidation and metabolic engineering. Molecular BioSystems 11, 38-59 (2015).

[38] Herbst, D. A., Townsend, C. A. \& Maier, T. The architectures of iterative type I PKS and FAS. Natural Product Reports 35, 1046-1069 (2018). 
[39] White, S. W., Zheng, J., Zhang, Y. M. \& Rock, C. O. The structural biology of type II fatty acid biosynthesis. Annual Review of Biochemistry 74, 791-831 (2005).

[40] Hiltunen, J. K. et al. Mitochondrial fatty acid synthesis and respiration. Biochimica et Biophysica Acta - Bioenergetics 1797, 1195-1202 (2010).

[41] Heil, C. S., Wehrheim, S. S., Paithankar, K. S. \& Grininger, M. Fatty Acid Biosynthesis: Chain-Length Regulation and Control. ChemBioChem 20, 2298-2321 (2019).

[42] Jenke-Kodama, H., Sandmann, A., Müller, R. \& Dittmann, E. Evolutionary implications of bacterial polyketide synthases. Molecular Biology and Evolution 22, 2027-2039 (2005).

[43] Hopwood, D. A. Genetic contributions to understanding polyketide synthases. Chemical Reviews 97, 2465-2497 (1997).

[44] Wang, B., Guo, F., Huang, C. \& Zhao, H. Unraveling the iterative type I polyketide synthases hidden in Streptomyces. Proceedings of the National Academy of Sciences of the United States of America 117, 8449-8454 (2020).

[45] Helfrich, E. J. N. \& Piel, J. Biosynthesis of polyketides by trans-AT polyketide synthases. Natural Product Reports 33, 231-316 (2016).

[46] Broadhurst, R. W., Nietlispach, D., Wheatcroft, M. P., Leadlay, P. F. \& Weissman, K. J. The Structure of Dockin Domains in Modular Polyketide Synthases. Chemistry \& Biology 10, 723-731 (2003).

[47] Dorival, J. et al. Characterization of Intersubunit Communication in the Virginiamycin trans-Acyl Transferase Polyketide Synthase. Journal of the American Chemical Society 138, 4155-4167 (2016).

[48] Funa, N. et al. A new pathway for polyketide synthesis in microorganisms. Nature 400, 897-899 (1999).

[49] Paitan, Y., Alon, G., Orr, E., Ron, E. Z. \& Rosenberg, E. The first gene in the biosynthesis of the polyketide antibiotic TA of Myxococcus xanthus codes for a unique PKS module coupled to a peptide synthetase. Journal of Molecular Biology 286, 465474 (1999).

[50] Shen, B. Polyketide biosynthesis beyond the type I, II and III polyketide synthase paradigms. Current Opinion in Chemical Biology 7, 285-295 (2003).

[51] Wakil, S. J. Fatty acid synthase, a proficient multifunctional enzyme. Biochemistry 28, 4523-4530 (1989). 
[52] Joshi, V. \& Wakil, S. J. Studies on the mechanism of fatty acid synthesis. XXVI. Purification and properties of malonyl-coenzyme A-acyl carrier protein transacylase of Escherichia coli. Archives of Biochemistry and Biophysics 143, 493-505 (1971).

[53] McCarthy, A. D. \& Grahame Hardie, D. Evidence that the acyl-O-esters are intermediates in the catalysis. The mechanism of rabbit mammary fatty acid synthase. FEBS Letters 150, 181-184 (1982).

[54] Majerus, P. W., Alberts, A. W. \& Vagelos, P. R. Acyl Carrier Protein, IV. the Identification of 4'-Phosphopantetheine As the Prosthetic Group of the Acyl Carrier Protein. Proceedings of the National Academy of Sciences 53, 410-417 (1965).

[55] Elovson, J. \& Vagelos, P. R. Acyl carrier protein. X. Acyl carrier protein synthetase. Journal of Biological Chemistry 243, 3603-3611 (1968).

[56] Smith, S., Witkowski, A. \& Joshi, A. K. Structural and functional organization of the animal fatty acid synthase. Progress in Lipid Research 42, 289-317 (2003).

[57] Crosby, J. \& Crump, M. P. The structural role of the carrier protein - Active controller or passive carrier. Natural Product Reports 29, 1111-1137 (2012).

[58] Arnstadt, K., Lynen, F. \& Schindlbeck, G. Zum Mechanismus der Kondensationsreaktion der Fettsäurebiosynthese. European Journal of Biochemistry 55, 561-571 (1975).

[59] Witkowski, A., Joshi, A. K. \& Smith, S. Characterization of the interthiol acyltranferase reaction catalyzed by the $\beta$-ketoacyl synthase domain of the animal fatty acid synthase. Biochemistry 41, 16338-16344 (1997).

[60] Witkowski, A., Joshi, A. K. \& Smith, S. Mechanism of the $\beta$-ketoacyl synthase reaction catalyzed by the animal fatty acid synthase. Biochemistry 41, 10877-10887 (2002).

[61] Price, A. C., Zhang, Y. M., Rock, C. O. \& White, S. W. Structure of $\beta$-ketoacyl-[acyl carrier protein] reductase from Escherichia coli: Negative cooperativity and its structural basis. Biochemistry 40, 12772-12781 (2001).

[62] Price, A. C., Zhang, Y. M., Rock, C. O. \& White, S. W. Cofactor-induced conformational rearrangements establish a catalytically competent active site and a proton relay conduit in FabG. Structure 12, 417-428 (2004).

[63] Medina, F. E., Neves, R. P., Ramos, M. J. \& Fernandes, P. A. A QM/MM study of the reaction mechanism of human $\beta$-ketoacyl reductase. Physical chemistry chemical physics 19, 347-355 (2016). 
[64] Leesong, M., Henderson, B. S., Gillig, J. R., Schwab, J. M. \& Smith, J. L. Structure of a dehydratase-isomerase from the bacterial pathway for biosynthesis of unsaturated fatty acids: Two catalytic activities in one active site. Structure 4, 253-264 (1996).

[65] Zhang, L. et al. Crystal structure of FabZ-ACP complex reveals a dynamic seesawlike catalytic mechanism of dehydratase in fatty acid biosynthesis. Cell Research 26, 1330-1344 (2016).

[66] Medina, F. E., Neves, R. P., Ramos, M. J. \& Fernandes, P. A. QM/MM Study of the Reaction Mechanism of the Dehydratase Domain from Mammalian Fatty Acid Synthase. ACS Catalysis 8, 10267-10278 (2018).

[67] Heath, R. J. \& Rock, C. O. Enoyl-acyl carrier protein reductase (fabl) plays a determinant role in completing cycles of fatty acid elongation in Escherichia coli. Journal of Biological Chemistry 270, 26538-26542 (1995).

[68] Sumper, M., Riepertinger, C., Lynen, F. \& Oesterhelt, D. Die Synthese verschiedener Carbonsäuren durch den Multienzymkomplex der Fettsäuresynthese aus Hefe und die Erklärung ihrer Bildung. European Journal of Biochemistry 10, 377-387 (1969).

[69] Maier, T., Jenni, S. \& Ban, N. Architecture of Mammalian Fatty Acid Synthase at 4.5 Å Resolution. Science 311, 1258-1262 (2006).

[70] Pollard, M. R., Anderson, L., Fan, C., Hawkins, D. J. \& Davies, H. M. A specific acyl-ACP thioesterase implicated in medium-chain fatty acid production in immature cotyledons of Umbellularia californica. Archives of Biochemistry and Biophysics 284, 306-312 (1991).

[71] Lu, Y. J. et al. Acyl-Phosphates Initiate Membrane Phospholipid Synthesis in GramPositive Pathogens. Molecular Cell 23, 765-772 (2006).

[72] Van Vranken, J. G. et al. ACP Acylation Is an Acetyl-CoA-Dependent Modification Required for Electron Transport Chain Assembly. Molecular Cell 71, 567-580 (2018).

[73] McDaniel, R., Ebert-Khosla, S., Hopwood, D. A. \& Khosla, C. Engineered biosynthesis of novel polyketides. Science 262, 1546-1550 (1993).

[74] Keatinge-Clay, A. T. The structures of type I polyketide synthases. Natural Product Reports 29, 1050-1073 (2012).

[75] Klaus, M. \& Grininger, M. Engineering strategies for rational polyketide synthase design. Natural Product Reports 35, 1070-1081 (2018). 
[76] Lowry, B., Li, X., Robbins, T., Cane, D. E. \& Khosla, C. A turnstile mechanism for the controlled growth of biosynthetic intermediates on assembly line polyketide synthases. ACS Central Science 2, 14-20 (2016).

[77] Kroken, S., Glass, N. L., Taylor, J. W., Yoder, O. C. \& Turgeon, B. G. Phylogenomic analysis of type I polyketide synthase genes in pathogenic and saprobic ascomycetes. Proceedings of the National Academy of Sciences of the United States of America 100, 15670-15675 (2003).

[78] Jenke-Kodama, H. \& Dittmann, E. Evolution of metabolic diversity: Insights from microbial polyketide synthases. Phytochemistry 70, 1858-1866 (2009).

[79] Ridley, C. P., Ho, Y. L. \& Khosla, C. Evolution of polyketide synthases in bacteria. Proceedings of the National Academy of Sciences of the United States of America 105, 4595-4600 (2008).

[80] Piel, J., Hui, D., Fusetani, N. \& Matsunaga, S. Targeting modular polyketide synthases with iteratively acting acyltransferases from metagenomes of uncultured bacterial consortia. Environmental Microbiology 6, 921-927 (2004).

[81] Lynen, F. Nobel Lecture - The pathway from "activated acetic acid" to the terpenes and fatty acids. http://biotools.nubic.northwestern.edu/OligoCalc.html (1964). NobelPrize.org. Nobel Media AB 2021. accessed 06.05.2021.

[82] Jenni, S. et al. Structure of Fungal Fatty Acid Synthase and Implications for Iterative Substrate Shuttling. Science 316, 254-261 (2007).

[83] Johansson, P. et al. Multimeric Options for the Auto-Activation of the Saccharomyces cerevisiae FAS Type I Megasynthase. Structure 17, 1063-1074 (2009).

[84] Boehringer, D., Ban, N. \& Leibundgut, M. 7.5-Å cryo-EM structure of the mycobacterial fatty acid synthase. Journal of Molecular Biology 425, 841-849 (2013).

[85] Maier, T., Leibundgut, M. \& Ban, N. The Crystal Structure of a Mammalian Fatty Acid Synthase. Science 321, 1315-1322 (2008).

[86] Grininger, M. Perspectives on the evolution, assembly and conformational dynamics of fatty acid synthase type I (FAS I) systems. Current Opinion in Structural Biology 25, 49-56 (2014).

[87] Weissman, K. J. Uncovering the structures of modular polyketide synthases. Natural Product Reports 32, 436-453 (2015). 
[88] Tsai, S. C. et al. Crystal structure of the macrocycle-forming thioesterase domain of the erythromycin polyketide synthase: Versatility from a unique substrate channel. Proceedings of the National Academy of Sciences of the United States of America 98, 14808-14813 (2001).

[89] Weissman, K. J. The structural biology of biosynthetic megaenzymes. Nature Chemical Biology 11, 660-670 (2015).

[90] Keatinge-Clay, A. T. \& Stroud, R. M. The Structure of a Ketoreductase Determines the Organization of the $\beta$-Carbon Processing Enzymes of Modular Polyketide Synthases. Structure 14, 737-748 (2006).

[91] Zheng, J., Fage, C. D., Demeler, B., Hoffman, D. W. \& Keatinge-Clay, A. T. The missing linker: A dimerization motif located within polyketide synthase modules. ACS Chemical Biology 8, 1263-1270 (2013).

[92] Gogos, A., Mu, H. \& Shapiro, L. Putative enoyl reductase domain of polyketide synthase. (PDB: 1PQW) (2003). URL https://www.rcsb.org/structure/1pqw.

[93] Keatinge-Clay, A. Crystal Structure of the Erythromycin Polyketide Synthase Dehydratase. Journal of Molecular Biology 384, 941-953 (2008).

[94] Akey, D. L. et al. Crystal Structures of Dehydratase Domains from the Curacin Polyketide Biosynthetic Pathway. Structure 18, 94-105 (2010).

[95] Tsai, S. C., Lu, H., Cane, D. E., Khosla, C. \& Stroud, R. M. Insights into channel architecture and substrate specificity from crystal structures of two macrocycle-forming thioesterases of modular polyketide synthases. Biochemistry 41, 12598-12606 (2002).

[96] Alekseyev, V. Y., Liu, C. W., Cane, D. E., Puglisi, J. D. \& Khosla, C. Solution structure and proposed domain domain recognition interface of an acyl carrier protein domain from a modular polyketide synthase. Protein Science 16, 2093-2107 (2007).

[97] Tran, L., Broadhurst, R. W., Tosin, M., Cavalli, A. \& Weissman, K. J. Insights into protein-protein and enzyme-substrate interactions in modular polyketide synthases. Chemistry and Biology 17, 705-716 (2010).

[98] Busche, A. et al. Characterization of molecular interactions between ACP and halogenase domains in the curacin A polyketide synthase. ACS Chemical Biology 7, 378-386 (2012).

[99] Tang, Y., Kim, C.-Y., Mathews, I. I., Cane, D. E. \& Khosla, C. The 2.7-Å crystal structure of a 194-kDa homodimeric fragment of the 6-deoxyerythronolide B synthase. PNAS 103, 11124-11129 (2006). 
[100] Tang, Y., Chen, A. Y., Kim, C. Y., Cane, D. E. \& Khosla, C. Structural and Mechanistic Analysis of Protein Interactions in Module 3 of the 6-Deoxyerythronolide B Synthase. Chemistry and Biology 14, 931-943 (2007).

[101] Zheng, J., Gay, D. C., Demeler, B., White, M. A. \& Keatinge-Clay, A. T. Divergence of multimodular polyketide synthases revealed by a didomain structure. Nature Chemical Biology 8, 615-621 (2012).

[102] Płoskoń, E. et al. A mammalian type I fatty acid synthase acyl carrier protein domain does not sequester acyl chains. Journal of Biological Chemistry 283, 518-528 (2008).

[103] Pappenberger, G. et al. Structure of the Human Fatty Acid Synthase KS-MAT Didomain as a Framework for Inhibitor Design. Journal of Molecular Biology 397, 508-519 (2010).

[104] Chakravarty, B., Gu, Z., Chirala, S. S., Wakil, S. J. \& Quiocho, F. A. Human fatty acid synthase: Structure and substrate selectivity of the thioesterase domain. Proceedings of the National Academy of Sciences of the United States of America 101, 15567-15572 (2004).

[105] Edwards, A. L., Matsui, T., Weiss, T. M. \& Khosla, C. Architectures of whole-module and bimodular proteins from the 6-deoxyerythronolide B synthase. Journal of Molecular Biology 426, 2229-2245 (2014).

[106] Dutta, S. et al. Structure of a modular polyketide synthase. Nature 510, 512-517 (2014).

[107] Whicher, J. R. et al. Structural rearrangements of a polyketide synthase module during its catalytic cycle. Nature 510, 560-564 (2014).

[108] Rittner, A. \& Grininger, M. Modular polyketide synthases (PKSs): A new model fits all? ChemBioChem 15, 2489-2493 (2014).

[109] D'Imprima, E. et al. Protein denaturation at the air-water interface and how to prevent it. elife 8, e42747 (2019).

[110] Herbst, D. A., Jakob, R. P., Zähringer, F. \& Maier, T. Mycocerosic acid synthase exemplifies the architecture of reducing polyketide synthases. Nature 531, 533-537 (2016).

[111] Gay, D., You, Y. O., Keatinge-Clay, A. \& Cane, D. E. Structure and stereospecificity of the dehydratase domain from the terminal module of the rifamycin polyketide synthase. Biochemistry 52, 8916-8928 (2013). 
[112] Fiers, W. D., Dodge, G. J., Sherman, D. H., Smith, J. L. \& Aldrich, C. C. Vinylogous Dehydration by a Polyketide Dehydratase Domain in Curacin Biosynthesis. Journal of the American Chemical Society 138, 16024-16036 (2016).

[113] Faille, A. et al. Insights into Substrate Modification by Dehydratases from Type I Polyketide Synthases. Journal of Molecular Biology 429, 1554-1569 (2017).

[114] Jakob, R., Herbst, D., Muller, R. \& Maier, T. Crystal Structures of Dehydratase Domains from trans-AT Polyketide Biosynthetic Pathway. (PDBs: 5J60, 5IL5, 5IL6, 5HU7, 5HQW) (2017). URL https://www.rcsb.org/structure/5J60.

[115] Keatinge-Clay, A. T. The Structural Relationship between Iterative and Modular PKSs. Cell Chemical Biology 23, 540-542 (2016).

[116] Ames, B. D. et al. Crystal structure and biochemical studies of the trans-acting polyketide enoyl reductase LovC from lovastatin biosynthesis. Proceedings of the National Academy of Sciences of the United States of America 109, 11144-11149 (2012).

[117] Khare, D. et al. Structural Basis for Cyclopropanation by a Unique Enoyl-Acyl Carrier Protein Reductase. Structure 23, 2213-2223 (2015).

[118] Wang, J. et al. Structural basis for the biosynthesis of lovastatin. Nature Communications 12: 867 (2021).

[119] Richter, C. D., Nietlispach, D., Broadhurst, R. W. \& Weissman, K. J. Multienzyme docking in hybrid megasynthetases. Nature Chemical Biology 4, 75-81 (2008).

[120] Buchholz, T. J. et al. Structural basis for binding specificity between subclasses of modular polyketide synthase docking domains. ACS Chemical Biology 4, 41-52 (2009).

[121] Whicher, J. R. et al. Cyanobacterial polyketide synthase docking domains: A tool for engineering natural product biosynthesis. Chemistry and Biology 20, 1340-1351 (2013).

[122] Reed, M. A. et al. The type I rat fatty acid synthase ACP shows structural homology and analogous biochemical properties to type II ACPs. Organic and Biomolecular Chemistry 1, 463-471 (2003).

[123] Andrec, M., Blake Hill, R. \& Prestegard, J. H. Amide exchange rates in Escherichia coli acyl carrier protein: Correlation with protein structure and dynamics. Protein Science 4, 983-993 (1995).

[124] Weissman, K. J., Hong, H., Popovic, B. \& Meersman, F. Evidence for a ProteinProtein Interaction Motif on an Acyl Carrier Protein Domain from a Modular Polyketide Synthase. Chemistry and Biology 13, 625-636 (2006). 
[125] Miyanaga, A., Iwasawa, S., Shinohara, Y., Kudo, F. \& Eguchi, T. Structure-based analysis of the molecular interactions between acyltransferase and acyl carrier protein in vicenistatin biosynthesis. Proceedings of the National Academy of Sciences of the United States of America 113, 1802-1807 (2016).

[126] Miyanaga, A. et al. Structural Basis of Protein-Protein Interactions between a transActing Acyltransferase and Acyl Carrier Protein in Polyketide Disorazole Biosynthesis. Journal of the American Chemical Society 140, 7970-7978 (2018).

[127] Cleland, W. W. The Kinetics of Enzyme-Catalyzed Reactions with Two or More Substrates or Products. I. Nomenclature and Rate Equations. Biochimica et Biophysica Acta 67, 104-137 (1963).

[128] Cleland, W. W. The Kinetics of Enzyme-Catalyzed Reactions with Two or More Substrates or Products. III. Prediction of Initial Velocity and Inhibition Patterns by Inspection. Biochimica et Biophysica Acta 67, 188-196 (1963).

[129] Copeland, R. A. Enzymes - A practical Introduction to Structure, Mechanism, and Data Analysis (Wiley-VCH, New York/ Chichester/Weinheim/ Brisbane/ Singapore/ Toronto, 2000), second edn.

[130] Bunkoczi, G. et al. Structural Basis for Different Specificities of Acyltransferases Associated with the Human Cytosolic and Mitochondrial Fatty Acid Synthases. Chemistry and Biology 16, 667-675 (2009).

[131] Cognet, J. A. \& Hammes, G. G. Elementary Steps in the Reaction Mechanism of Chicken Liver Fatty Acid Synthase: Acetylation-Deacetylation. Biochemistry 22, 30023007 (1983).

[132] Bonnett, S. A. et al. Acyl-CoA subunit selectivity in the pikromycin polyketide synthase PikAIV: Steady-state kinetics and active-site occupancy analysis by FTICR-MS. Chemistry and Biology 18, 1075-1081 (2011).

[133] Dunn, B. J., Cane, D. E. \& Khosla, C. Mechanism and Specificity of an Acyltransferase Domain from a Modular Polyketide Synthase. Biochemistry 52, 1839-1841 (2013).

[134] Paiva, P., Sousa, S. F., Ramos, M. J. \& Fernandes, P. A. Understanding the Catalytic Machinery and the Reaction Pathway of the Malonyl-Acetyl Transferase Domain of Human Fatty Acid Synthase. ACS Catalysis 8, 4860-4872 (2018).

[135] Paiva, P., Sousa, S. F., Fernandes, P. A. \& João Ramos, M. Human Fatty Acid Synthase: A Computational Study of the Transfer of the Acyl Moieties from MAT to the ACP Domain. ChemCatChem 11, 3853-3864 (2019). 
[136] Serre, L., Verbree, E. C., Dauter, Z., Stuitje, A. R. \& Derewenda, Z. S. The Escherichia coli malonyl-CoA:acyl carrier protein transacylase at $1.5-\AA$ resolution: Crystal structure of a fatty acid synthase component. Journal of Biological Chemistry 270, 12961-12964 (1995).

[137] Keatinge-Clay, A. T. et al. Catalysis, specificity, and ACP docking site of Streptomyces coelicolor malonyl-CoA:ACP Transacylase. Structure 11, 147-154 (2003).

[138] Smith, S. \& Stern, A. The Effect of Aromatic CoA Esters on Fatty Acid Synthetase: Biosynthesis of $\omega$-Phenyl Fatty Acids. Archives of Biochemistry and Biophysics 222, 259-265 (1983).

[139] Liou, G. F., Lau, J., Cane, D. E. \& Khosla, C. Quantitative analysis of loading and extender acyltransferases of modular polyketide synthases. Biochemistry 42, 200-207 (2003).

[140] Wang, F. et al. Structural and functional analysis of the loading acyltransferase from avermectin modular polyketide synthase. ACS Chemical Biology 10, 1017-1025 (2015).

[141] Koryakina, I., McArthur, J. B., Draelos, M. M. \& Williams, G. J. Promiscuity of a modular polyketide synthase towards natural and non-natural extender units. Organic \& Biomolecular Chemistry 11, 4449-4458 (2013).

[142] Dunn, B. J., Watts, K. R., Robbins, T., Cane, D. E. \& Khosla, C. Comparative Analysis of the Substrate Specificity of trans- versus cis-Acyltransferases of Assembly Line Polyketide Synthases. Biochemistry 53, 3796-3806 (2014).

[143] Geyer, K., Sundaram, S., Sušnik, P., Koert, U. \& Erb, T. J. Understanding Substrate Selectivity of Phoslactomycin Polyketide Synthase by Using Reconstituted in Vitro Systems. ChemBioChem 21, 2080 - 2085 (2020).

[144] Haydock, S. F. et al. Divergent sequence motifs correlated with the substrate specificity of (methyl)malonyl-CoA:acyl carrier protein transacylase domains in modular polyketide synthases. FEBS Letters 374, 246-248 (1995).

[145] Yadav, G., Gokhale, R. S. \& Mohanty, D. Computational approach for prediction of domain organization and substrate specificity of modular polyketide synthases. Journal of Molecular Biology 328, 335-363 (2003).

[146] Nardini, M. \& Dijkstra, B. W. $\alpha / \beta$ hydrolase fold enzymes: The family keeps growing. Current Opinion in Structural Biology 9, 732-737 (1999). 
[147] Reeves, C. D. et al. Alteration of the substrate specificity of a modular polyketide synthase acyltransferase domain through site-specific mutations. Biochemistry 40, 1546415470 (2001).

[148] Del Vecchio, F. et al. Active-site residue, domain and module swaps in modular polyketide synthases. Journal of Industrial Microbiology and Biotechnology 30, 489-494 (2003).

[149] Lau, J., Fu, H., Cane, D. E. \& Khosla, C. Dissecting the role of acyltransferase domains of modular polyketide synthases in the choice and stereochemical fate of extender units. Biochemistry 38, 1643-1651 (1999).

[150] Heath, R. J. \& Rock, C. O. The Claisen condensation in biology. Natural Product Reports 19, 581-596 (2002).

[151] Huang, W. et al. Crystal structure of $\beta$-ketoacyl-acyl carrier protein synthase II from E.coli reveals the molecular architecture of condensing enzymes. EMBO Journal 17, 1183-1191 (1998).

[152] Von Wettstein-Knowles, P., Olsen, J. G., McGuire, K. A. \& Henriksen, A. Fatty acid synthesis: Role of active site histidines and lysine in Cys-His-His-type $\beta$-ketoacyl-acyl carrier protein synthases. FEBS Journal 273, 695-710 (2006).

[153] Blaquiere, N., Shore, D. G., Rousseaux, S. \& Fagnou, K. Decarboxylative ketone aldol reactions: Development and mechanistic evaluation under metal-free conditions. Journal of Organic Chemistry 74, 6190-6198 (2009).

[154] Cui, W., Liang, Y., Tian, W., Ji, M. \& Ma, X. Regulating effect of $\beta$-ketoacyl synthase domain of fatty acid synthase on fatty acyl chain length in de novo fatty acid synthesis. Biochimica et Biophysica Acta - Molecular and Cell Biology of Lipids 1861, 149-155 (2016).

[155] Yalpani, M., Willecke, K. \& Lynen, F. Triacetic Acid Lactone, a Derailment Product of Fatty Acid Biosynthesis. European Journal of Biochemistry 8, 495-502 (1969).

[156] Seyama, Y., Otsuka, H., Kawaguchi, A. \& Yamakawa, T. Fatty acid synthetase from the harderian gland of Guinea pig: Biosynthesis of methyl-branched fatty acids. Journal of Biochemistry 90, 789-798 (1981).

[157] Dewulf, J. P. et al. The synthesis of branched-chain fatty acids is limited by enzymatic decarboxylation of ethyl- and methylmalonyl-CoA. Biochemical Journal 476, 2427-2447 (2019). 
[158] Khosla, C., Gokhale, R. S., Jacobsen, J. R. \& Cane, D. E. Tolerance and Specificity of Polyketide Synthases. Annual Review of Biochemistry 68, 219-253 (1999).

[159] Viegas, M. F., Neves, R. P., Ramos, M. J. \& Fernandes, P. A. Modeling of Human Fatty Acid Synthase and in Silico Docking of Acyl Carrier Protein Domain and Its Partner Catalytic Domains. Journal of Physical Chemistry B 122, 77-85 (2018).

[160] Milligan, J. C. et al. Molecular basis for interactions between an acyl carrier protein and a ketosynthase. Nature Chemical Biology 15, 669-671 (2019).

[161] Mindrebo, J. T. et al. Gating mechanism of elongating $\beta$-ketoacyl-ACP synthases. Nature Communications 11: 1727 (2020).

[162] Kapur, S., Chen, A. Y., Cane, D. E. \& Khosla, C. Molecular recognition between ketosynthase and acyl carrier protein domains of the 6-deoxyerythronolide B synthase. Proceedings of the National Academy of Sciences of the United States of America 107, 22066-22071 (2010).

[163] Klaus, M., D'Souza, A. D., Nivina, A., Khosla, C. \& Grininger, M. Engineering of Chimeric Polyketide Synthases Using SYNZIP Docking Domains. ACS Chemical Biology 14, 426-433 (2019).

[164] Peng, H., Ishida, K., Sugimoto, Y., Jenke-Kodama, H. \& Hertweck, C. Emulating evolutionary processes to morph aureothin-type modular polyketide synthases and associated oxygenases. Nature Communications 10: 3918 (2019).

[165] Zhang, L. et al. Characterization of Giant Modular PKSs Provides Insight into Genetic Mechanism for Structural Diversification of Aminopolyol Polyketides. Angewandte Chemie - International Edition 56, 1740-1745 (2017).

[166] Vander Wood, D. A. \& Keatinge-Clay, A. T. The modules of trans-acyltransferase assembly lines redefined with a central acyl carrier protein. Proteins: Structure, Function and Bioinformatics 86, 664-675 (2018).

[167] Keatinge-Clay, A. T. Polyketide Synthase Modules Redefined. Angewandte Chemie International Edition 56, 4658-4660 (2017).

[168] Weissman, K. J. Genetic engineering of modular PKSs: from combinatorial biosynthesis to synthetic biology. Natural Product Reports 33, 203-230 (2016).

[169] Woodward, R. B. et al. Asymmetric Total Synthesis of Erythromycin. 1. Synthesis of an Erythronolide A Seco Acid Derivative via Asymmetric Induction. Journal of the American Chemical Society 103, 3210-3213 (1981). 
[170] Woodward, R. B. et al. Asymmetric Total Synthesis of Erythromyin. 3. Total Synthesis of Erythromycin. Journal of the American Chemical Society 103, 3215-3217 (1981).

[171] Woodward, R. B. et al. Asymmetric Total Synthesis of Erythromycin. 2. Synthesis of an Erythronolide A Lactone System. Journal of the American Chemical Society 103, 3213-3215 (1981).

[172] Malpartida, D. \& Hopwood, D. A. Molecular cloning of the whole biosynthetic pathways of a Streptomyces antibiotic and its expression in a heterologous host. Nature 309, 462464 (1984).

[173] Bartel, P. L. et al. Biosynthesis of anthraquinones by interspecies cloning of actinorhodin biosynthesis genes in streptomycetes: Clarification of actinorhodin gene functions. Journal of Bacteriology 172, 4816-4826 (1990).

[174] McDaniel, R., Kao, C. M., Hwang, S. J. \& Khosla, C. Engineered intermodular and intramodular polyketide synthase fusions. Chemistry and Biology 4, 667-674 (1997).

[175] Donadio, S., Staver, M. J., McAlpine, J. B., Swanson, S. J. \& Katz, L. Modular organization of genes required for complex polyketide biosynthesis. Science 252, 675679 (1991).

[176] Carreras, C. W. \& Santi, D. V. Engineering of modular polyketide synthases to produce novel polyketides. Current Opinion in Biotechnology 9, 403-411 (1998).

[177] Verdine, G. L. The combinatorial chemistry of nature. Nature 384, 11-13 (1996).

[178] Menzella, H. G. et al. Combinatorial polyketide biosynthesis by de novo design and rearrangement of modular polyketide synthase genes. Nature Biotechnology 23, 11711176 (2005).

[179] Kao, C. M., Khosla, C., Luo, G., Cane, D. E. \& Katz, L. Engineered biosynthesis of a triketide lactone from an incomplete modular polyketide synthase. Journal of the American Chemical Society 116, 11612-11613 (1994).

[180] Cortes, J. et al. Repositioning of a domain in a modular polyketide synthase to promote specific chain cleavage. Science 268, 1487-1489 (1995).

[181] Donadio, S., McAlpine, J. B., Sheldon, P. J., Jackson, M. \& Katz, L. An erythromycin analog produced by reprogramming of polyketide synthesis. Proceedings of the National Academy of Sciences of the United States of America 90, 7119-7123 (1993).

[182] Jacobsen, J. R., Hutchinson, C. R., Cane, D. E. \& Khosla, C. Precursor-directed biosynthesis of erythromycin analogs by an engineered polyketide synthase. Science 277, 367-369 (1997). 
[183] Oliynyk, M., Brown, M. J., Cortes, J., Staunton, J. \& Leadlay, P. F. A hybrid modular polyketide synthase obtained by domain swapping. Chemistry and Biology 3, 833-839 (1996).

[184] Kuhstoss, S., Huber, M., Turner, J. R., Paschal, J. W. \& Rao, R. N. Production of a novel polyketide through the construction of a hybrid polyketide synthase. Gene 183, 231-236 (1996).

[185] Liu, L., Thamchaipenet, A., Fu, H., Betlach, M. \& Ashley, G. Biosynthesis of 2nor-6-deoxyerythronolide $B$ by rationally designed domain substitution. Journal of the American Chemical Society 119, 10553-10554 (1997).

[186] McDaniel, R. et al. Gain-of-Function Mutagenesis of a Modular Polyketide Synthase. Journal of the American Chemical Society 119, 4309-4310 (1997).

[187] Bravo-Rodriguez, K. et al. Substrate Flexibility of a Mutated Acyltransferase Domain and Implications for Polyketide Biosynthesis. Chemistry and Biology 22, 1425-1430 (2015).

[188] Murphy, A. C., Hong, H., Vance, S., Broadhurst, R. W. \& Leadlay, P. F. Broadening substrate specificity of a chain-extending ketosynthase through a single active-site mutation. Chemical Communications 52, 8373-8376 (2016).

[189] Xue, Q., Ashley, G., Hutchinson, C. R. \& Santi, D. V. A multiplasmid approach to preparing large libraries of polyketides. Proceedings of the National Academy of Sciences of the United States of America 96, 11740-11745 (1999).

[190] Kim, B. S., Sherman, D. H. \& Reynolds, K. A. An efficient method for creation and functional analysis of libraries of hybrid type I polyketide synthases. Protein Engineering, Design and Selection 17, 277-284 (2004).

[191] Long, P. F. et al. Engineering specificity of starter unit selection by the erythromycinproducing polyketide synthase. Molecular Microbiology 43, 1215-1225 (2002).

[192] Rowe, C. J. et al. Engineering a polyketide with a longer chain by insertion of an extra module into the erythromycin-producing polyketide synthase. Chemistry and Biology $\mathbf{8}$, 475-485 (2001).

[193] Martin, C. J. et al. Heterologous expression in Saccharopolyspora erythraea of a pentaketide synthase derived from the spinosyn polyketide synthase. Organic and Biomolecular Chemistry 1, 4144-4147 (2003). 
[194] Gaisser, S. et al. Direct production of ivermectin-like drugs after domain exchange in the avermectin polyketide synthase of Streptomyces avermitilis ATCC31272. Organic and Biomolecular Chemistry 1, 2840-2847 (2003).

[195] Reeves, C. D. et al. Production of Hybrid 16-Membered Macrolides by Expressing Combinations of Polyketide Synthase Genes in Engineered Streptomyces fradiae Hosts. Chemistry and Biology 11, 1465-1472 (2004).

[196] Barajas, J. F., Blake-Hedges, J. M., Bailey, C. B., Curran, S. \& Keasling, J. D. Engineered polyketides: Synergy between protein and host level engineering. Synthetic and Systems Biotechnology 2, 147-166 (2017).

[197] Mcdaniel, R. et al. Multiple genetic modifications of the erythromycin polyketide synthase to produce a library of novel "unnatural" natural products. Proceedings of the National Academy of Sciences of the United States of America 96, 1846-1851 (1999).

[198] Kushnir, S. et al. Minimally invasive mutagenesis gives rise to a biosynthetic polyketide library. Angewandte Chemie - International Edition 51, 10664-10669 (2012).

[199] Klaus, M. et al. Protein-protein interactions, not substrate recognition, dominate the turnover of chimeric assembly line polyketide synthases. Journal of Biological Chemistry 291, 16404-16415 (2016).

[200] Holzbaur, I. E. et al. Molecular basis of Celmer's rules: Role of the ketosynthase domain in epimerisation and demonstration that ketoreductase domains can have altered product specificity with unnatural substrates. Chemistry and Biology 8, 329-340 (2001).

[201] Kellenberger, L. et al. A polylinker approach to reductive loop swaps in modular polyketide synthases. Chembiochem : a European journal of chemical biology 9, 2740-2749 (2008).

[202] Sugimoto, Y., Ding, L., Ishida, K. \& Hertweck, C. Rational design of modular polyketide synthases: Morphing the aureothin pathway into a luteoreticulin assembly line. Angewandte Chemie - International Edition 53, 1560-1564 (2014).

[203] Awakawa, T. et al. Reprogramming of the antimycin NRPS-PKS assembly lines inspired by gene evolution. Nature Communications 9: 3534 (2018).

[204] Chemler, J. A. et al. Evolution of Efficient Modular Polyketide Synthases by Homologous Recombination. Journal of the American Chemical Society 137, 10603-10609 (2015).

[205] Wlodek, A. et al. Diversity oriented biosynthesis via accelerated evolution of modular gene clusters. Nature Communications 8: 1206 (2017). 
[206] Miyazawa, T., Hirsch, M., Zhang, Z. \& Keatinge-clay, A. T. An in vitro platform for engineering and harnessing modular polyketide synthases. Nature Communications 11: 80 (2020).

[207] Reinke, A. W., Grant, R. A. \& Keating, A. E. A synthetic coiled-coil interactome provides heterospecific modules for molecular engineering. Journal of the American Chemical Society 132, 6025-6031 (2010).

[208] Thompson, K. E., Bashor, C. J., Lim, W. A. \& Keating, A. E. Synzip protein interaction toolbox: In vitro and in vivo specifications of heterospecific coiled-coil interaction domains. ACS Synthetic Biology 1, 118-129 (2012).

[209] Klaus, M., Buyachuihan, L. \& Grininger, M. Ketosynthase Domain Constrains the Design of Polyketide Synthases. ACS Chemical Biology 15, 2422-2432 (2020).

[210] Stegemann, F. Fluorescence Studies on Dynamic Domains of the Mammalian Fatty Acid Synthase and Different Polyketide Synthases. Master's thesis, Goethe-University Frankfurt (2016).

[211] Molnos, J., Gardiner, R., Dale, G. E. \& Lange, R. A continuous coupled enzyme assay for bacterial malonyl-CoA:acyl carrier protein transacylase (FabD). Analytical Biochemistry 319, 171-176 (2003).

[212] Rittner, A., Paithankar, K. S., Himmler, A. \& Grininger, M. Type I fatty acid synthase trapped in the octanoyl-bound state. Protein Science 29, 589-605 (2020).

[213] Dimroth, P., Rlingelmann, E. \& Lynen, F. 6-Methylsalicylic Acid Synthetase from Penicillium patulum Some Catalytic Properties of the Enzyme and Its Relation to Fatty Acid Synthetase. European Journal of Biochemistry 68, 591-596 (1976).

[214] Oliynyk, M. et al. Complete genome sequence of the erythromycin-producing bacterium Saccharopolyspora erythraea NRRL23338. Nature Biotechnology 25, 447-453 (2007).

[215] Moriguchi, T., Kezuka, Y., Nonaka, T., Ebizuka, Y. \& Fujii, I. Hidden function of catalytic domain in 6-methylsalicylic acid synthase for product release. Journal of Biological Chemistry 285, 15637-15643 (2010).

[216] Marcella, A. M. \& Barb, A. W. The R117A variant of the Escherichia coli transacylase FabD synthesizes novel acyl-(acyl carrier proteins). Applied Microbiology and Biotechnology 101, 8431-8441 (2017).

[217] Liew, C. W. et al. Crystal structure of the acyltransferase domain of the iterative polyketide synthase in enediyne biosynthesis. Journal of Biological Chemistry 287, 23203-23215 (2012). 
[218] Wang, Y.-Y. et al. Biochemical Characterization of a Malonyl-Specific Acyltransferase Domain of FK506 Biosynthetic Polyketide Synthase. Protein \& Peptide Letters 22, 2-7 (2015).

[219] Takamura, Y. \& Nomura, G. Changes in the intracellular concentration of acetyl-CoA and malonyl-CoA in relation to the carbon and energy metabolism of Escherichia coli K12. Journal of general microbiology 134, 2249-2253 (1988).

[220] Davis, M. S., Solbiati, J. \& Cronan, J. E. Overproduction of acetyl-CoA carboxylase activity increases the rate of fatty acid biosynthesis in Escherichia coli. Journal of Biological Chemistry 275, 28593-28598 (2000).

[221] Rossini, E., Gajewski, J., Klaus, M., Hummer, G. \& Grininger, M. Analysis and engineering of substrate shuttling by the acyl carrier protein (ACP) in fatty acid synthases (FASs). Chemical Communications 54, 11606-11609 (2018).

[222] Heil, C. S., Rittner, A., Goebel, B., Beyer, D. \& Grininger, M. Site-Specific Labelling of Multidomain Proteins by Amber Codon Suppression. Scientific Reports 8: 14864 (2018).

[223] Berlinghof, M. Analyse von Domänen-Domänen-Interaktionen in Megasynthasen. Master's thesis, Goethe-University Frankfurt (2018).

[224] Chari, A. et al. ProteoPlex: Stability optimization of macromolecular complexes by sparse-matrix screening of chemical space. Nature Methods 12, 859-865 (2015).

[225] Mayer, S., Rüdiger, S., Ang, H. C., Joerger, A. C. \& Fersht, A. R. Correlation of Levels of Folded Recombinant p53 in Escherichia coli with Thermodynamic Stability in Vitro. Journal of Molecular Biology 372, 268-276 (2007).

[226] Just, A. Analyse von Fettsäure- und Polyketidsynthase-Hybriden in Struktur und Funktion. Master's thesis, Goethe-University Frankfurt (2020).

[227] Szafranska, A. E., Hitchman, T. S., Cox, R. J., Crosby, J. \& Simpson, T. J. Kinetic and mechanistic analysis of the malonyl CoA:ACP transacylase from Streptomyces coelicolor indicates a single catalytically competent serine nucleophile at the active site. Biochemistry 41, 1421-1427 (2002).

[228] Lowe, P. N. \& Rhodes, S. Purification and characterization of [acyl-carrier-protein] acetyltransferase from Escherichia coli. The Biochemical journal 250, 789-796 (1988).

[229] Bar-Even, A. et al. The moderately efficient enzyme: Evolutionary and physicochemical trends shaping enzyme parameters. Biochemistry 50, 4402-4410 (2011). 
[230] Rangan, V. S. \& Smith, S. Expression in Escherichia coli and refolding of the malonyl/acetyltransferase domain of the multifunctional animal fatty acid synthase. Journal of Biological Chemistry 271, 31749-31755 (1996).

[231] Albery, W. J. \& Knowles, J. R. Evolution of Enzyme Function and the Development of Catalytic Efficiency. Biochemistry 15, 5631-5640 (1976).

[232] Lowry, B. et al. In vitro reconstitution and analysis of the 6-deoxyerythronolide B synthase. Journal of the American Chemical Society 135, 16809-16812 (2013).

[233] Grininger, M. The role of the iterative modules in polyketide synthase evolution. Proceedings of the National Academy of Sciences of the United States of America 117, 8680-8682 (2020).

[234] Musiol-Kroll, E. M. \& Wohlleben, W. Acyltransferases as tools for polyketide synthase engineering. Antibiotics 7: 62 (2018).

[235] Gajewski, J. et al. Engineering fatty acid synthases for directed polyketide production. Nature Chemical Biology 13, 363-365 (2017).

[236] Kumar, S. \& Nussinov, R. Close-range electrostatic interactions in proteins. ChemBioChem 3, 604-617 (2002).

[237] Zhou, H. X. \& Pang, X. Electrostatic Interactions in Protein Structure, Folding, Binding, and Condensation. Chemical Reviews 118, 1691-1741 (2018).

[238] Khersonsky, O. \& Tawfik, D. S. Enzyme promiscuity: A mechanistic and evolutionary perspective. Annual Review of Biochemistry 79, 471-505 (2010).

[239] Kim, C. Y. et al. Reconstituting modular activity from separated domains of 6deoxyerythronolide B synthase. Biochemistry 43, 13892-13898 (2004).

[240] Torres, J. P., Lin, Z., Winter, J. M., Krug, P. J. \& Schmidt, E. W. Animal biosynthesis of complex polyketides in a photosynthetic partnership. Nature Communications 11: 2882 (2020).

[241] Richardson, M. T., Pohl, N. L., Kealey, J. T. \& Khosla, C. Tolerance and specificity of recombinant 6-methylsalicylic acid synthase. Metabolic Engineering 1, 180-187 (1999).

[242] Jensen, R. A. Enzyme recruitment in evolution of new function. Annual review of microbiology 30, 409-425 (1976).

[243] Kalkreuter, E., Crowetipton, J. M., Lowell, A. N., Sherman, D. H. \& Williams, G. J. Engineering the Substrate Specificity of a Modular Polyketide Synthase for Installation 


\section{References}

of Consecutive Non-Natural Extender Units. Journal of the American Chemical Society 141, 1961-1969 (2019).

[244] Kibbe, W. A. Oligocalc calculator version 3.27. http://biotools.nubic. northwestern.edu/OligoCalc.html.

[245] Xie, D. et al. Microbial synthesis of triacetic acid lactone. Biotechnology and Bioengineering 93, 727-736 (2006).

[246] Waterhouse, A. et al. SWISS-MODEL: Homology modelling of protein structures and complexes. Nucleic Acids Research 46, W296-W303 (2018).

[247] Krissinel, E. \& Henrick, K. Inference of macromolecular assemblies from crystalline state. Journal of Molecular Biology 273, 774-797 (2007).

[248] Xue, L. C., Rodrigues, J. P., Kastritis, P. L., Bonvin, A. M. \& Vangone, A. PRODIGY: A web server for predicting the binding affinity of protein-protein complexes. Bioinformatics 32, 3676-3678 (2016).

[249] Barlow, K. A. et al. Flex ddG: Rosetta Ensemble-Based Estimation of Changes in Protein-Protein Binding Affinity upon Mutation. Journal of Physical Chemistry B 122, 5389-5399 (2018).

[250] Kortemme, T., Kim, D. E. \& Baker, D. Computational alanine scanning of proteinprotein interfaces. Science Signaling 2004, pl2-pl2 (2004).

[251] Dittrich, J., Schmidt, D., Pfleger, C. \& Gohlke, H. Converging a Knowledge-Based Scoring Function: DrugScore 2018. Journal of Chemical Information and Modeling 59, 509-521 (2019).

[252] Pires, D. E., Ascher, D. B. \& Blundell, T. L. MCSM: Predicting the effects of mutations in proteins using graph-based signatures. Bioinformatics 30, 335-342 (2014).

[253] Moreira, I. S. et al. SpotOn: High Accuracy Identification of Protein-Protein Interface Hot-Spots. Scientific Reports 7: 8007 (2017).

[254] Valenzano, C. R. et al. Stereospecificity of the dehydratase domain of the erythromycin polyketide synthase. Journal of the American Chemical Society 132, 14697-14699 (2010).

[255] Peter, D. M. et al. Screening and Engineering the Synthetic Potential of Carboxylating Reductases from Central Metabolism and Polyketide Biosynthesis. Angewandte Chemie - International Edition 54, 13457-13461 (2015). 


\section{References}

[256] Fahim, A. Modulation einer AT:ACP-Interaktionsfläche der 6-Desoxyerythronolid BSynthase. Bachelor's thesis, Goethe-University Frankfurt (2019). 


\section{Supplementary Information}

\section{Exemplary Plasmid Map}

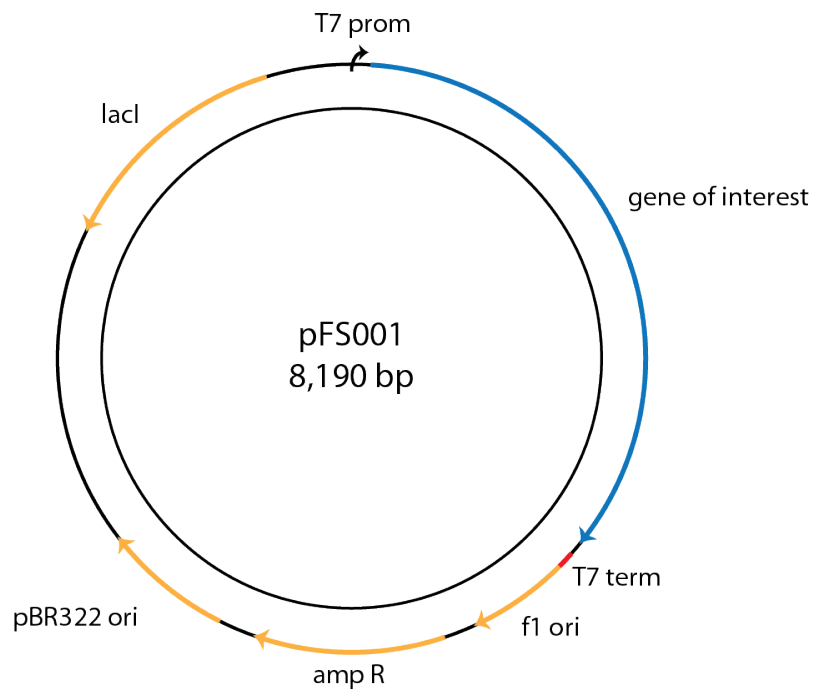

Plasmid map of pFS001 representing all pET22b vector constructs. Vectors contain an $\mathrm{f} 1$ and a pBR322 origin, an ampicillin resistance, the gene of interest encoding the respective protein, and the gene lacl, which controls protein expression via the T7 polymerase system. 


\section{Excerpt of Plasmids}

Plasmids pAR and pMJD were kindly provided by Dr. Alexander Rittner and Mirko Joppe. The gene encoding for EcPKS1 was kindly provided by Eric Schmidt Lab (University of Utah). The gene encoding for Npt from Streptomyces platensis was kindly provided by Kyra Geyer, who is part of the Tobias Erb Lab (MPI Marburg).

List of plasmids for wildtype AT characterization.

\begin{tabular}{ll}
\hline$\#$ & Name \\
\hline- & Sfp_Bsub_pETcoco \\
pAR090 & Strepl_Ecoli(FabD)_H8_pET22b \\
pAR225 & Strepl_Pks7(KS(C161G)-AT)_H8_pET22b \\
pAR318 & Strepl_AVES1(ACP)0_H8_pET22b \\
pAR319 & Strepl_AVES1(AT)0_H8_pET22b \\
pAR331 & Strepl_DEBS3(ACP)5_H8_pET22b \\
pAR332 & Strepl_PikAIII(ACP)5_H8_pET22b \\
pAR333 & Strepl_RAPS3(ACP)14_H8_pET22b \\
pAR357 & Sfp_Bsub_pCDF-1b \\
\hline pFS001 & Strepl_DEBS3(KS(C233G)-AT)5_H8_pET22b \\
pFS004 & Strepl_PikAIII(KS(C243G)-AT)5_H8_pET22b \\
pFS007 & Strepl_RAPS(KS(C226G)-AT)14_H8_pET22b \\
pFS011 & Ecoli(ACP)_H8_pET22b \\
pFS025 & Strepl_DEBS1(ACP)0_H8_pET22b \\
pFS026 & Strepl_Pks7(ACP)_H8_pET22b \\
pFS030 & Strepl_DEBS1(AT)0_MBP_H8_pET22b \\
pFS120 & Strepl_DEBS3(ACP)5_COP_H8_pET22b \\
pFS121 & Strepl_PikAIII(ACP)5_COP_H8_pET22b \\
pFS122 & Strepl_RAPS3(ACP)14_COP_H8_pET22b \\
pFS123 & Strepl_Pks7(ACP)_COP_H8_pET22b \\
pFS124 & Strepl_Ecoli(ACP)_COP_H8_pET22b \\
pFS125 & Strepl_DEBS1(ACP)0_COP_H8_pET22b \\
pFS126 & Strepl_AVES1(ACP)0_COP_H8_pET22b \\
pFS129 & Strepl_DEBS3(KS(C233G)-AT(A539S))5_H8_pET22b \\
pFS130 & Strepl_DEBS3(KS(C233G)-AT(A539D))5_H8_pET22b \\
pFS131 & Strepl_DEBS3(KS(C233G)-AT(A539E))5_H8_pET22b \\
pFS132 & Strepl_DEBS3(KS(C233G)-AT(A539F))5_H8_pET22b \\
pFS133 & Strepl_DEBS3(KS(C233G)-AT(R850K))5_H8_pET22b \\
pFS134 & Strepl_DEBS3(KS(C233G)-AT(R850A))5_H8_pET22b \\
pFS135 & Strepl_DEBS3(KS(C233G)-AT(R850E))5_H8_pET22b \\
pFS136 & Strepl_DEBS3(KS(C233G)-AT(R850F))5_H8_pET22b \\
pFS137 & Strepl_DEBS3(KS(C233G)-AT(R850F))5_H8_pET22b \\
\hline &
\end{tabular}


List of plasmids for construction of FAS chimeras.

\begin{tabular}{|c|c|}
\hline$\#$ & Name \\
\hline pAR069 & Strepl_m(KS-LD-AT)_H8_pET22b \\
\hline pAR265 & Strepl_DEBS3(KS-LD-AT)5_H8_pET22b \\
\hline pAR352B & StreplI_m(ACP)_H8_RBS_Sfp_Bsub_pET22b \\
\hline pFS031 & Strepl_m(KS-LD)-DEBS3(AT)5-m(DH-ER-KR-ACP)_H8_pET22b \\
\hline pFS032 & Strepl_m(KS)-DEBS3(LD-AT)5-m(DH-ER-KR-ACP)_H8_pET22b \\
\hline pFS033 & Strepl_m(KS)-DEBS3(LD-AT)5-m(DH-ER-KR-ACP)_H8_pET22b \\
\hline pFS034 & Strepl_m(KS-LD)-RAPS3(AT)14-m(DH-ER-KR-ACP)_H8_pET22b \\
\hline pFS035 & Strepl_m(KS)-RAPS3(LD-AT)14-m(DH-ER-KR-ACP)_H8_pET22b \\
\hline pFS036 & Strepl_m(KS-LD)-RAPS3(LD-AT)14-m(DH-ER-KR-ACP)_H8_pET22b \\
\hline pFS037 & Strepl_m(KS-LD)-DEBS3(AT)5_H8_pET22b \\
\hline pFS038 & Strepl_m(KS)-DEBS3(LD-AT)5_H8_pET22b \\
\hline pFS039 & Strepl_m(KS(D61A)-LD-AT)_H8_pET22b \\
\hline pFS040 & Strepl_m(KS(N96A)-LD-AT)_H8_pET22b \\
\hline pFS041 & Strepl_m(KS-LD(R429A)-AT)_H8_pET22b \\
\hline pFS042 & Strepl_m(KS-LD(I457A)-AT)_H8_pET22b \\
\hline pFS043 & Strepl_m(KS-LD(R468A)-AT)_H8_pET22b \\
\hline pFS044 & Strepl_m(KS-LD(Y470A)-AT)_H8_pET22b \\
\hline pFS045 & Strepl_DEBS3(KS(E129A)-LD-AT)5_H8_pET22b \\
\hline pFS046 & Strepl_DEBS3(KS(D134A)-LD-AT)5_H8_pET22b \\
\hline pFS047 & Strepl_DEBS3(KS-LD(R510A)-AT)5_H8_pET22b \\
\hline pFS048 & Strepl_DEBS3(KS-LD(V518A)-AT)5_H8_pET22b \\
\hline pFS049 & Strepl_DEBS3(KS-LD-AT(Y867A))5_H8_pET22b \\
\hline pFS050 & Strepl_DEBS3(KS-LD-AT(A868R))5_H8_pET22b \\
\hline pFS051 & Strepl_m(KS-LD(H425A)-AT)_H8_pET22b \\
\hline pFS052 & Strepl_m(KS-LD(V479A)-AT)_H8_pET22b \\
\hline pFS053 & Strepl_m(KS-LD(E481A)-AT)_H8_pET22b \\
\hline pFS054 & Strepl_m(KS-LD(Q483A)-AT)_H8_pET22b \\
\hline pFS055 & Strepl_m(KS-LD-AT(H804A))_H8_pET22b \\
\hline pFS056 & Strepl_m(KS-LD-AT(L805A))_H8_pET22b \\
\hline pFS057 & Strepl_m(KS-LD)-DEBS3(AT)5_H8_pET22b \\
\hline pFS058 & Strepl_m(KS-LD)-DEBS3(AT)5_H8_pET22b \\
\hline pFS059 & Strepl_m(KS-LD)-DEBS3(AT)5_H8_pET22b \\
\hline pFS060 & Strepl_m(KS)-DEBS3(LD-AT)5_H8_pET22b \\
\hline pFS061 & Strepl_m(KS)-DEBS3(LD-AT)5_H8_pET22b \\
\hline pFS062 & Strepl_m(KS)-DEBS3(LD-AT)5_H8_pET22b \\
\hline pFS116 & Strepl_m(KS-LD)-Ecoli(FabD)_H8_pET22b \\
\hline pFS117 & Strepl_m(KS-LD)-Ecoli(FabD)_H8_pET22b \\
\hline pFS118 & Strepl_m(KS-LD)-DEBS3(AT)6_H8_pET22b \\
\hline pFS119 & Strepl_m(KS-LD)-DEBS3(AT)6_H8_pET22b \\
\hline pFS138 & Strepl_DEBS3(KS-LD-AT)6_H8_pET22b \\
\hline pFS139 & Strepl_DEBS3(KS-LD)6-m(AT)_H8_pET22b \\
\hline pFS163 & Strepl_DEBS(DD)5-m(KS-LD)-VemG(AT-ACP)-SZ3_H6_pET22b \\
\hline pFS164 & Strepl_DEBS(DD)5-m(KS)-VemG(LD-AT-ACP)-SZ3_H6_pET22b \\
\hline pFS165 & Strepl_DEBS(DD)5-m(KS-LD)-VemH(AT-ACP)-SZ3_H6_pET22b \\
\hline pFS166 & Strepl_DEBS(DD)5-m(KS)-VemH(LD-AT-ACP)-SZ3_H6_pET22b \\
\hline pFS167 & Strepl_DEBS(DD)5-m(KS-LD)-DEBS3(AT)6-m(L-ACP)-SZ3_H6_pET22b \\
\hline pFS168 & Strepl_DEBS(DD)5-m(KS-LD)-DEBS3(AT)6-m(L)-DEBS3(ACP)6-SZ3_H6_pET22b \\
\hline pFS170 & Strepl_DEBS(DD)5-m(KS-LD)-EcPKS1(AT-L-ACP)-SZ3_H6_pET22b \\
\hline pFS171 & Strepl_DEBS(DD)5-m(KS)-EcPKS1(LD-AT-L-ACP)-SZ3_H6_pET22b \\
\hline pFS172 & Strepl_DEBS(DD)5-m(KS)-EcPKS1(LD-AT-L-ACP)-SZ3_H6_pET22b \\
\hline pFS173 & Strepl_DEBS(DD)5-EcPKS1(KS-LD-AT-L-ACP)-SZ3_H6_pET22b \\
\hline pMJD091 & Npt_Splat_pCDF-1b \\
\hline pMJD094 & H6_DEBS3(ACP)6_pET28a(+) \\
\hline
\end{tabular}




\section{Amino Acid Sequences of Chimeras}

FS031 Strepl-m(KS-LD)-DEBS3(AT)5-m(DH-ER-KR-ACP)-H8

MSAWSHPQFEKGGGSGGGSGGSAWSHPQFEKAAAEEVVIAGMSGKLPESENLQEFWANLIGGVDMVTDDD RRWKAGLYGLPKRSGKLKDLSKFDASFFGVHPKQAHTMDPQLRLLLEVSYEAIVDGGINPASLRGTNTGVW VGVSGSEASEALSRDPETLLGYSMVGCQRAMMANRLSFFFDFKGPSIALDTACSSSLLALQNAYQAIRSGEC PAALVGGINLLLKPNTSVQFMKLGMLSPDGTCRSFDDSGSGYCRSEAVVAVLLTKKSLARRVYATILNAGTN TDGSKEQGVTFPSGEVQEQLICSLYQPAGLAPESLEYIEAHGTGTKVGDPQELNGITRSLCAFRQAPLLIGST KSNMGHPEPASGLAALTKVLLSLEHGVWAPNLHFHNPNPEIPALLDGRLQVVDRPLPVRGGNVGINSFGFGG SNVHVILQPNTRQAPAPTAHAALPHLLHASGRTLEAVQDLLEQGRQHSQDLAFVSMLNDIAATPTAAMPFR GYTVLGVEGRVQEVQQVSTRRGVAMVFPGQGAQWQGMARDLLRESQVFADSIRDCERALAPHVDW SLTDLLSGARPLDRVDVVQPALFAVMVSLAALWRSHGVEPAAVVGHSQGEIAAAHVAGALTLEDAA KLVAVRSRVLRRLGGQGGMASFGLGTEQAAERIGRFAGALSIASVNGPRSVVVAGESGPLDELIAEC EAEGITARRIPVDYASHSPQVESLREELLTELAGISPVSADVALYSTTTGQPIDTATMDTAYWYANL REQVRFQDATRQLAEAGFDAFVEVSPHPVLTVGIEATLDSALPADAGACVVGTLRRDRGGLADFH

TALGEAYAQGINVNPNALFPPVEFPAPRGTPLISPHIKWDHSQTWDVPVAEDFPNGSSSSSATVYSIDASPE SPDHYLVDHCIDGRVIFPGTGYLCLVWKTLARSLGLSLEETPVVFENVSFHQATILPKTGTVALEVRLLEASH AFEVSDTGNLIVSGKVYLWEDPNSKLFDHPEVPTPPESASVSRLTQGEVYKELRLRGYDYGPQFQGICEATL EGEQGKLLWKDNWVTFMDTMLQVSILGSSQQSLQLPTRVTAIYIDPATHRQKVYRLKEDTQVADVTTSRC LGITVSGGIHISRLQTTATSRRQQEQLVPTLEKFVFTPHMEAECLSESTALQKELQLCKGLARALQTKATQQ GLKAAMLGQEDPPQHGLPRLLAAACQLQLNGNLQLELGEALAQERLLLPEDPLISGLLNSQALKACVDTALE NLSTLKMKVAEVLAGEGHLYSRIPALLNTQPMLQLEYTATDRHPQALKDVQTKLQQHDVAQGQWNPSDPA PSSLGALDLLVCNCALATLGDPALALDNMVAALKEGGFLLVHTVLKGHALGETLACLPSEVQPAPSLLSQEE WESLFSRKALHLVGLKRSFYGTALFLCRRAIPQEKPIFLSVEDTSFQWVDSLKSTLATSSSQPVWLTAMDCP TSGVVGLVNCLRKEPGGHRIRCILLSNLSNTSHAPKLDPGSPELQQVLKHDLVMNVYRDGAWGAFRHFQLE QDKPKEQTAHAFVNVLTRGDLASIRWVSSPLKHTQPSSSGAQLCTVYYASLNFRDIMLATGKLSPDAIPGK WASRDCMLGMEFSGRDRCGRRVMGLVPAEGLATSVLLSSDFLWDVPSSWTLEEAASVPVVYTTAYYSLVV RGRIQRGETVLIHSGSGGVGQAAISIALSLGCRVFTTVGSAEKRAYLQARFPQLDDTSFANSRDTSFEQHVLL HTGGKGVDLVLNSLAEEKLQASVRCLAQHGRFLEIGKFDLSNNHPLGMAIFLKNVTFHGILLDALFEEANDS WREVAALLKAGIRDGVVKPLKCTVFPKAQVEDAFRYMAQGKHIGKVLVQVREEEPEAVLPGAQPTLISAISK TFCPAHKSYIITGGLGGFGLELARWLVLRGAQRLVLTSRSGIRTGYQAKHIREWRRQGIQVLVSTSNVSSLEGA RALIAEATKLGPVGGVFNLAMVLRDAMLENQTPELFQDVNKPKYNGTLNLDRATREACPELDYFVAFSSVS CGRGNAGQTNYGFANSTMERICEQRRHDGLPGLAVQWGAIGDVGIVLEAMGTNDTVIGGTLPQRISSCMEV LDLFLNQPHAVLSSFVLAEKKAVAHGDGDTQRDLVKAVAHILGIRDLAGINLDSTLADLGLDSLMGVEVRQIL EREHDLVLPMREVRQLTLRKLQEMSSKTDSATDTTAPKSRSDTSLKQNQLNLSTLLVNPEGPTLTQLNSVQ SSERPLFLVHPIEGSTTVFHSLAAKLSVPTYGLQCTQAAPLDSIPNLAAYYIDCIKQVQPEGPYRIAGYSFGAC VAFEMCSQLQAQQGPAPTHNNLFLFDGSHTYVLAYTQSYRAKMTPGCEAEAEAEALCFFIKQFLDVEHSKV LEALLPLKSLEDRVAASVDLITKSHHSLDRRELSFAAVSFYHKLRAADQYKPKAKYHGNVTLLRAKTGGTY GEDLGADYNLSQVCDGKVSVHIIEGDHRTLLEGSGLESIINIIHSSLAEPRVSVREGLEHHHHHHHH 
FS032 Strepl-m(KS)-DEBS3(LD-AT)5-m(DH-ER-KR-ACP)-H8

MSAWSHPQFEKGGGSGGGSGGSAWSHPQFEKAAAEEVVIAGMSGKLPESENLQEFWANLIGGVDMVTDDD RRWKAGLYGLPKRSGKLKDLSKFDASFFGVHPKQAHTMDPQLRLLLEVSYEAIVDGGINPASLRGTNTGVW VGVSGSEASEALSRDPETLLGYSMVGCQRAMMANRLSFFFDFKGPSIALDTACSSSLLALQNAYQAIRSGEC PAALVGGINLLLKPNTSVQFMKLGMLSPDGTCRSFDDSGSGYCRSEAVVAVLLTKKSLARRVYATILNAGTN TDGSKEQGVTFPSGEVQEQLICSLYQPAGLAPESLEYIEAHGTGTKVGDPQELNGITRSLCAFRQAPLLIGST KSNMGHPEPASGLAALTKVLLSLEHGVWAPNLHFHNPNPEIPALLDGRLQVVDRPLPVRGGNVGINSFGFGG SNVHVILQPNEADEPEPAPDSGPVPLVLSGRDEQAMRAQAGRLADHLAREPRNSLRDTGFTLATRR SAWEHRAVVVGDRDDALAGLRAVADGRIADRTATGQARTRRGVAMVFPGQGAQWQGMARDLLR ESQVFADSIRDCERALAPHVDWSLTDLLSGARPLDRVDVVQPALFAVMVSLAALWRSHGVEPAAV VGHSQGEIAAAHVAGALTLEDAAKLVAVRSRVLRRLGGQGGMASFGLGTEQAAERIGRFAGALSIAS VNGPRSVVVAGESGPLDELIAECEAEGITARRIPVDYASHSPQVESLREELLTELAGISPVSADVALYS TTTGQPIDTATMDTAYWYANLREQVRFQDATRQLAEAGFDAFVEVSPHPVLTVGIEATLDSALPA DAGACVVGTLRRDRGGLADFHTALGEAYAQGVEVDWSPAFAPVEFPAPRGTPLISPHIKWDHSQTWD VPVAEDFPNGSSSSSATVYSIDASPESPDHYLVDHCIDGRVIFPGTGYLCLVWKTLARSLGLSLEETPVVFEN VSFHQATILPKTGTVALEVRLLEASHAFEVSDTGNLIVSGKVYLWEDPNSKLFDHPEVPTPPESASVSRLTQ GEVYKELRLRGYDYGPQFQGICEATLEGEQGKLLWKDNWVTFMDTMLQVSILGSSQQSLQLPTRVTAIYID PATHRQKVYRLKEDTQVADVTTSRCLGITVSGGIHISRLQTTATSRRQQEQLVPTLEKFVFTPHMEAECLSE STALQKELQLCKGLARALQTKATQQGLKAAMLGQEDPPQHGLPRLLAAACQLQLNGNLQLELGEALAQER LLLPEDPLISGLLNSQALKACVDTALENLSTLKMKVAEVLAGEGHLYSRIPALLNTQPMLQLEYTATDRHPQ ALKDVQTKLQQHDVAQGQWNPSDPAPSSLGALDLLVCNCALATLGDPALALDNMVAALKEGGFLLVHTVL KGHALGETLACLPSEVQPAPSLLSQEEWESLFSRKALHLVGLKRSFYGTALFLCRRAIPQEKPIFLSVEDTSFQ WVDSLKSTLATSSSQPVWLTAMDCPTSGVVGLVNCLRKEPGGHRIRCILLSNLSNTSHAPKLDPGSPELQQV LKHDLVMNVYRDGAWGAFRHFQLEQDKPKEQTAHAFVNVLTRGDLASIRWVSSPLKHTQPSSSGAQLCTV YYASLNFRDIMLATGKLSPDAIPGKWASRDCMLGMEFSGRDRCGRRVMGLVPAEGLATSVLLSSDFLWDVP SSWTLEEAASVPVVYTTAYYSLVVRGRIQRGETVLIHSGSGGVGQAAISIALSLGCRVFTTVGSAEKRAYLQA RFPQLDDTSFANSRDTSFEQHVLLHTGGKGVDLVLNSLAEEKLQASVRCLAQHGRFLEIGKFDLSNNHPLG MAIFLKNVTFHGILLDALFEEANDSWREVAALLKAGIRDGVVKPLKCTVFPKAQVEDAFRYMAQGKHIGKV LVQVREEEPEAVLPGAQPTLISAISKTFCPAHKSYIITGGLGGFGLELARWLVLRGAQRLVLTSRSGIRTGYQA KHIREWRRQGIQVLVSTSNVSSLEGARALIAEATKLGPVGGVFNLAMVLRDAMLENQTPELFQDVNKPKYN GTLNLDRATREACPELDYFVAFSSVSCGRGNAGQTNYGFANSTMERICEQRRHDGLPGLAVQWGAIGDVGI VLEAMGTNDTVIGGTLPQRISSCMEVLDLFLNQPHAVLSSFVLAEKKAVAHGDGDTQRDLVKAVAHILGIRDL AGINLDSTLADLGLDSLMGVEVRQILEREHDLVLPMREVRQLTLRKLQEMSSKTDSATDTTAPKSRSDTSLK QNQLNLSTLLVNPEGPTLTQLNSVQSSERPLFLVHPIEGSTTVFHSLAAKLSVPTYGLQCTQAAPLDSIPNLA AYYIDCIKQVQPEGPYRIAGYSFGACVAFEMCSQLQAQQGPAPTHNNLFLFDGSHTYVLAYTQSYRAKMTP GCEAEAEAEALCFFIKQFLDVEHSKVLEALLPLKSLEDRVAASVDLITKSHHSLDRRELSFAAVSFYHKLRAA DQYKPKAKYHGNVTLLRAKTGGTYGEDLGADYNLSQVCDGKVSVHIIEGDHRTLLEGSGLESIINIIHSSLAE PRVSVREGLEHHHHHHHH 
FS033 Strepl-m(KS)-DEBS3(LD-AT)5-m(DH-ER-KR-ACP)-H8

MSAWSHPQFEKGGGSGGGSGGSAWSHPQFEKAAAEEVVIAGMSGKLPESENLQEFWANLIGGVDMVTDDD RRWKAGLYGLPKRSGKLKDLSKFDASFFGVHPKQAHTMDPQLRLLLEVSYEAIVDGGINPASLRGTNTGVW VGVSGSEASEALSRDPETLLGYSMVGCQRAMMANRLSFFFDFKGPSIALDTACSSSLLALQNAYQAIRSGEC PAALVGGINLLLKPNTSVQFMKLGMLSPDGTCRSFDDSGSGYCRSEAVVAVLLTKKSLARRVYATILNAGTN TDGSKEQGVTFPSGEVQEQLICSLYQPAGLAPESLEYIEAHGTGTKVGDPQELNGITRSLCAFRQAPLLIGST KSNMGHPEPASGLAALTKVLLSLEHGVWAPNLHFHNPNPEIPALLDGRLQVVDRPLPVRGGNVGINSFGFGG SNVHVILQPNEADEPEPAPDSGPVPLVLSGRDEQAMRAQAGRLADHLAREPRNSLRDTGFTLATRR SAWEHRAVVVGDRDDALAGLRAVADGRIADRTATGQARTRRGVAMVFPGQGAQWQGMARDLLR ESQVFADSIRDCERALAPHVDWSLTDLLSGARPLDRVDVVQPALFAVMVSLAALWRSHGVEPAAV VGHSQGEIAAAHVAGALTLEDAAKLVAVRSRVLRRLGGQGGMASFGLGTEQAAERIGRFAGALSIAS VNGPRSVVVAGESGPLDELIAECEAEGITARRIPVDYASHSPQVESLREELLTELAGISPVSADVALYS TTTGQPIDTATMDTAYWYANLREQVRFQDATRQLAEAGFDAFVEVSPHPVLTVGIEATLDSALPA DAGACVVGTLRRDRGGLADFHTALGEAYAQGVEVDWSPAFADARPVEPLISPHIKWDHSQTWDVPV AEDFPNGSSSSSATVYSIDASPESPDHYLVDHCIDGRVIFPGTGYLCLVWKTLARSLGLSLEETPVVFENVSF HQATILPKTGTVALEVRLLEASHAFEVSDTGNLIVSGKVYLWEDPNSKLFDHPEVPTPPESASVSRLTQGEV YKELRLRGYDYGPQFQGICEATLEGEQGKLLWKDNWVTFMDTMLQVSILGSSQQSLQLPTRVTAIYIDPAT HRQKVYRLKEDTQVADVTTSRCLGITVSGGIHISRLQTTATSRRQQEQLVPTLEKFVFTPHMEAECLSESTA LQKELQLCKGLARALQTKATQQGLKAAMLGQEDPPQHGLPRLLAAACQLQLNGNLQLELGEALAQERLLL PEDPLISGLLNSQALKACVDTALENLSTLKMKVAEVLAGEGHLYSRIPALLNTQPMLQLEYTATDRHPQALK KDVQTKLQQHDVAQGQWNPSDPAPSSLGALDLLVCNCALATLGDPALALDNMVAALKEGGFLLVHTVLKG HALGETLACLPSEVQPAPSLLSQEEWESLFSRKALHLVGLKRSFYGTALFLCRRAIPQEKPIFLSVEDTSFQW VDSLKSTLATSSSQPVWLTAMDCPTSGVVGLVNCLRKEPGGHRIRCILLSNLSNTSHAPKLDPGSPELQQVL KHDLVMNVYRDGAWGAFRHFQLEQDKPKEQTAHAFVNVLTRGDLASIRWVSSPLKHTQPSSSGAQLCTVY YASLNFRDIMLATGKLSPDAIPGKWASRDCMLGMEFSGRDRCGRRVMGLVPAEGLATSVLLSSDFLWDVPS SWTLEEAASVPVVYTTAYYSLVVRGRIQRGETVLIHSGSGGVGQAAISIALSLGCRVFTTVGSAEKRAYLQAR FPQLDDTSFANSRDTSFEQHVLLHTGGKGVDLVLNSLAEEKLQASVRCLAQHGRFLEIGKFDLSNNHPLGM AIFLKNVTFHGILLDALFEEANDSWREVAALLKAGIRDGVVKPLKCTVFPKAQVEDAFRYMAQGKHIGKVLV QVREEEPEAVLPGAQPTLISAISKTFCPAHKSYIITGGLGGFGLELARWLVLRGAQRLVLTSRSGIRTGYQAKHI REWRRQGIQVLVSTSNVSSLEGARALIAEATKLGPVGGVFNLAMVLRDAMLENQTPELFQDVNKPKYNGTL NLDRATREACPELDYFVAFSSVSCGRGNAGQTNYGFANSTMERICEQRRHDGLPGLAVQWGAIGDVGIVLEA MGTNDTVIGGTLPQRISSCMEVLDLFLNQPHAVLSSFVLAEKKAVAHGDGDTQRDLVKAVAHILGIRDLAGIN LDSTLADLGLDSLMGVEVRQILEREHDLVLPMREVRQLTLRKLQEMSSKTDSATDTTAPKSRSDTSLKQNQ LNLSTLLVNPEGPTLTQLNSVQSSERPLFLVHPIEGSTTVFHSLAAKLSVPTYGLQCTQAAPLDSIPNLAAYY IDCIKQVQPEGPYRIAGYSFGACVAFEMCSQLQAQQGPAPTHNNLFLFDGSHTYVLAYTQSYRAKMTPGCE AEAEAEALCFFIKQFLDVEHSKVLEALLPLKSLEDRVAASVDLITKSHHSLDRRELSFAAVSFYHKLRAADQY KPKAKYHGNVTLLRAKTGGTYGEDLGADYNLSQVCDGKVSVHIIEGDHRTLLEGSGLESIINIIHSSLAEPRV SVREGLEHHHHHHHH 
FS034 Strepl-m(KS-LD)-RAPS3(AT)14-m(DH-ER-KR-ACP)-H8

MSAWSHPQFEKGGGSGGGSGGSAWSHPQFEKAAAEEVVIAGMSGKLPESENLQEFWANLIGGVDMVTDDD RRWKAGLYGLPKRSGKLKDLSKFDASFFGVHPKQAHTMDPQLRLLLEVSYEAIVDGGINPASLRGTNTGVW VGVSGSEASEALSRDPETLLGYSMVGCQRAMMANRLSFFFDFKGPSIALDTACSSSLLALQNAYQAIRSGEC PAALVGGINLLLKPNTSVQFMKLGMLSPDGTCRSFDDSGSGYCRSEAVVAVLLTKKSLARRVYATILNAGTN TDGSKEQGVTFPSGEVQEQLICSLYQPAGLAPESLEYIEAHGTGTKVGDPQELNGITRSLCAFRQAPLLIGST KSNMGHPEPASGLAALTKVLLSLEHGVWAPNLHFHNPNPEIPALLDGRLQVVDRPLPVRGGNVGINSFGFGG SNVHVILQPNTRQAPAPTAHAALPHLLHASGRTLEAVQDLLEQGRQHSQDLAFVSMLNDIAATPTAAMPFR GYTVLGVEGRVQEVQQVSTARRVAFLFDGQGTQRLGMGKELYDSYPAFARAWDTVSAGFDKHLDH SLTDVCFGEGGSTTAGLVDDTLYAQAGIFAMEAALFGLLEDWGVRPDFVAGHSIGEATAAYASGML SLENVTTLIVARGRALRTTPPGAMVALRAGEEEVREFLSRTGAALDLAAVNSPEAVVVSGEPEPVA DFEAAWTASGREARKLKVRHAFHSRHVEAVLDEFRTALESLKFRAPALPVVSTVTGRLIDQDEMG TPEYWLRQVRRPVRFQDAVRELAEQGVGTFVEVGPSGALASAGVECLGGDASFHAVLRPRSPEDV CLMTAIAELHAGGINVNPNALFPPVEFPAPRGTPLISPHIKWDHSQTWDVPVAEDFPNGSSSSSATVYSID ASPESPDHYLVDHCIDGRVIFPGTGYLCLVWKTLARSLGLSLEETPVVFENVSFHQATILPKTGTVALEVRLL EASHAFEVSDTGNLIVSGKVYLWEDPNSKLFDHPEVPTPPESASVSRLTQGEVYKELRLRGYDYGPQFQGIC EATLEGEQGKLLWKDNWVTFMDTMLQVSILGSSQQSLQLPTRVTAIYIDPATHRQKVYRLKEDTQVADVT TSRCLGITVSGGIHISRLQTTATSRRQQEQLVPTLEKFVFTPHMEAECLSESTALQKELQLCKGLARALQTK ATQQGLKAAMLGQEDPPQHGLPRLLAAACQLQLNGNLQLELGEALAQERLLLPEDPLISGLLNSQALKACV DTALENLSTLKMKVAEVLAGEGHLYSRIPALLNTQPMLQLEYTATDRHPQALKDVQTKLQQHDVAQGQWN PSDPAPSSLGALDLLVCNCALATLGDPALALDNMVAALKEGGFLLVHTVLKGHALGETLACLPSEVQPAPSLL SQEEWESLFSRKALHLVGLKRSFYGTALFLCRRAIPQEKPIFLSVEDTSFQWVDSLKSTLATSSSQPVWLTA MDCPTSGVVGLVNCLRKEPGGHRIRCILLSNLSNTSHAPKLDPGSPELQQVLKHDLVMNVYRDGAWGAFRH FQLEQDKPKEQTAHAFVNVLTRGDLASIRWVSSPLKHTQPSSSGAQLCTVYYASLNFRDIMLATGKLSPDAI PGKWASRDCMLGMEFSGRDRCGRRVMGLVPAEGLATSVLLSSDFLWDVPSSWTLEEAASVPVVYTTAYYS LVVRGRIQRGETVLIHSGSGGVGQAAISIALSLGCRVFTTVGSAEKRAYLQARFPQLDDTSFANSRDTSFEQH VLLHTGGKGVDLVLNSLAEEKLQASVRCLAQHGRFLEIGKFDLSNNHPLGMAIFLKNVTFHGILLDALFEEAN DSWREVAALLKAGIRDGVVKPLKCTVFPKAQVEDAFRYMAQGKHIGKVLVQVREEEPEAVLPGAQPTLISAI SKTFCPAHKSYIITGGLGGFGLELARWLVLRGAQRLVLTSRSGIRTGYQAKHIREWRRQGIQVLVSTSNVSSLE GARALIAEATKLGPVGGVFNLAMVLRDAMLENQTPELFQDVNKPKYNGTLNLDRATREACPELDYFVAFSS VSCGRGNAGQTNYGFANSTMERICEQRRHDGLPGLAVQWGAIGDVGIVLEAMGTNDTVIGGTLPQRISSCM EVLDLFLNQPHAVLSSFVLAEKKAVAHGDGDTQRDLVKAVAHILGIRDLAGINLDSTLADLGLDSLMGVEVRQ ILEREHDLVLPMREVRQLTLRKLQEMSSKTDSATDTTAPKSRSDTSLKQNQLNLSTLLVNPEGPTLTQLNSV QSSERPLFLVHPIEGSTTVFHSLAAKLSVPTYGLQCTQAAPLDSIPNLAAYYIDCIKQVQPEGPYRIAGYSFGA CVAFEMCSQLQAQQGPAPTHNNLFLFDGSHTYVLAYTQSYRAKMTPGCEAEAEAEALCFFIKQFLDVEHSK VLEALLPLKSLEDRVAASVDLITKSHHSLDRRELSFAAVSFYHKLRAADQYKPKAKYHGNVTLLRAKTGGTY GEDLGADYNLSQVCDGKVSVHIIEGDHRTLLEGSGLESIINIIHSSLAEPRVSVREGLEHHHHHHHH 
FS035 Strepl-m(KS)-RAPS3(LD-AT)14-m(DH-ER-KR-ACP)-H8

MSAWSHPQFEKGGGSGGGSGGSAWSHPQFEKAAAEEVVIAGMSGKLPESENLQEFWANLIGGVDMVTDDD RRWKAGLYGLPKRSGKLKDLSKFDASFFGVHPKQAHTMDPQLRLLLEVSYEAIVDGGINPASLRGTNTGVW VGVSGSEASEALSRDPETLLGYSMVGCQRAMMANRLSFFFDFKGPSIALDTACSSSLLALQNAYQAIRSGEC PAALVGGINLLLKPNTSVQFMKLGMLSPDGTCRSFDDSGSGYCRSEAVVAVLLTKKSLARRVYATILNAGTN TDGSKEQGVTFPSGEVQEQLICSLYQPAGLAPESLEYIEAHGTGTKVGDPQELNGITRSLCAFRQAPLLIGST KSNMGHPEPASGLAALTKVLLSLEHGVWAPNLHFHNPNPEIPALLDGRLQVVDRPLPVRGGNVGINSFGFGG SNVHVILQPNAPPVTPVTPVTPNEPGPLPWVLSAQSPKALREQAGRLYASLAGDSEWNSLDIGYSL ATTRSDFAHRAVAVGSGREDFLRALSKLADGAPWPGLTTATATAKARRVAFLFDGQGTQRLGMGK ELYDSYPAFARAWDTVSAGFDKHLDHSLTDVCFGEGGSTTAGLVDDTLYAQAGIFAMEAALFGLLE DWGVRPDFVAGHSIGEATAAYASGMLSLENVTTLIVARGRALRTTPPGAMVALRAGEEEVREFLSR TGAALDLAAVNSPEAVVVSGEPEPVADFEAAWTASGREARKLKVRHAFHSRHVEAVLDEFRTALE SLKFRAPALPVVSTVTGRLIDQDEMGTPEYWLRQVRRPVRFQDAVRELAEQGVGTFVEVGPSGA LASAGVECLGGDASFHAVLRPRSPEDVCLMTAIAELHAGGTAIDWAKVLSPVEFPAPRGTPLISPHIK WDHSQTWDVPVAEDFPNGSSSSSATVYSIDASPESPDHYLVDHCIDGRVIFPGTGYLCLVWKTLARSLGLSL EETPVVFENVSFHQATILPKTGTVALEVRLLEASHAFEVSDTGNLIVSGKVYLWEDPNSKLFDHPEVPTPPE SASVSRLTQGEVYKELRLRGYDYGPQFQGICEATLEGEQGKLLWKDNWVTFMDTMLQVSILGSSQQSLQLP TRVTAIYIDPATHRQKVYRLKEDTQVADVTTSRCLGITVSGGIHISRLQTTATSRRQQEQLVPTLEKFVFTPH MEAECLSESTALQKELQLCKGLARALQTKATQQGLKAAMLGQEDPPQHGLPRLLAAACQLQLNGNLQLEL GEALAQERLLLPEDPLISGLLNSQALKACVDTALENLSTLKMKVAEVLAGEGHLYSRIPALLNTQPMLQLEYT ATDRHPQALKDVQTKLQQHDVAQGQWNPSDPAPSSLGALDLLVCNCALATLGDPALALDNMVAALKEGG FLLVHTVLKGHALGETLACLPSEVQPAPSLLSQEEWESLFSRKALHLVGLKRSFYGTALFLCRRAIPQEKPIFL SVEDTSFQWVDSLKSTLATSSSQPVWLTAMDCPTSGVVGLVNCLRKEPGGHRIRCILLSNLSNTSHAPKLDP GSPELQQVLKHDLVMNVYRDGAWGAFRHFQLEQDKPKEQTAHAFVNVLTRGDLASIRWVSSPLKHTQPSS SGAQLCTVYYASLNFRDIMLATGKLSPDAIPGKWASRDCMLGMEFSGRDRCGRRVMGLVPAEGLATSVLLS SDFLWDVPSSWTLEEAASVPVVYTTAYYSLVVRGRIQRGETVLIHSGSGGVGQAAISIALSLGCRVFTTVGSA EKRAYLQARFPQLDDTSFANSRDTSFEQHVLLHTGGKGVDLVLNSLAEEKLQASVRCLAQHGRFLEIGKFDL SNNHPLGMAIFLKNVTFHGILLDALFEEANDSWREVAALLKAGIRDGVVKPLKCTVFPKAQVEDAFRYMAQ GKHIGKVLVQVREEEPEAVLPGAQPTLISAISKTFCPAHKSYIITGGLGGFGLELARWLVLRGAQRLVLTSRSGI RTGYQAKHIREWRRQGIQVLVSTSNVSSLEGARALIAEATKLGPVGGVFNLAMVLRDAMLENQTPELFQDV NKPKYNGTLNLDRATREACPELDYFVAFSSVSCGRGNAGQTNYGFANSTMERICEQRRHDGLPGLAVQWG AIGDVGIVLEAMGTNDTVIGGTLPQRISSCMEVLDLFLNQPHAVLSSFVLAEKKAVAHGDGDTQRDLVKAVA HILGIRDLAGINLDSTLADLGLDSLMGVEVRQILEREHDLVLPMREVRQLTLRKLQEMSSKTDSATDTTAPKS RSDTSLKQNQLNLSTLLVNPEGPTLTQLNSVQSSERPLFLVHPIEGSTTVFHSLAAKLSVPTYGLQCTQAAP LDSIPNLAAYYIDCIKQVQPEGPYRIAGYSFGACVAFEMCSQLQAQQGPAPTHNNLFLFDGSHTYVLAYTQS YRAKMTPGCEAEAEAEALCFFIKQFLDVEHSKVLEALLPLKSLEDRVAASVDLITKSHHSLDRRELSFAAVSF YHKLRAADQYKPKAKYHGNVTLLRAKTGGTYGEDLGADYNLSQVCDGKVSVHIIEGDHRTLLEGSGLESII NIIHSSLAEPRVSVREGLEHHHHHHHH 
FS036 Strepl-m(KS-LD)-RAPS3(LD-AT)14-m(DH-ER-KR-ACP)-H8

MSAWSHPQFEKGGGSGGGSGGSAWSHPQFEKAAAEEVVIAGMSGKLPESENLQEFWANLIGGVDMVTDDD RRWKAGLYGLPKRSGKLKDLSKFDASFFGVHPKQAHTMDPQLRLLLEVSYEAIVDGGINPASLRGTNTGVW VGVSGSEASEALSRDPETLLGYSMVGCQRAMMANRLSFFFDFKGPSIALDTACSSSLLALQNAYQAIRSGEC PAALVGGINLLLKPNTSVQFMKLGMLSPDGTCRSFDDSGSGYCRSEAVVAVLLTKKSLARRVYATILNAGTN TDGSKEQGVTFPSGEVQEQLICSLYQPAGLAPESLEYIEAHGTGTKVGDPQELNGITRSLCAFRQAPLLIGST KSNMGHPEPASGLAALTKVLLSLEHGVWAPNLHFHNPNPEIPALLDGRLQVVDRPLPVRGGNVGINSFGFGG SNVHVILQPNAPPVTPVTPVTPNEPGPLPWVLSAQSPKALREQAGRLYASLAGDSEWNSLDIGYSL ATTRSDFAHRAVAVGSGREDFLRALSKLADGAPWPGLTTATATAKARRVAFLFDGQGTQRLGMGK ELYDSYPAFARAWDTVSAGFDKHLDHSLTDVCFGEGGSTTAGLVDDTLYAQAGIFAMEAALFGLLE DWGVRPDFVAGHSIGEATAAYASGMLSLENVTTLIVARGRALRTTPPGAMVALRAGEEEVREFLSR TGAALDLAAVNSPEAVVVSGEPEPVADFEAAWTASGREARKLKVRHAFHSRHVEAVLDEFRTALE SLKFRAPALPVVSTVTGRLIDQDEMGTPEYWLRQVRRPVRFQDAVRELAEQGVGTFVEVGPSGA LASAGVECLGGDASFHAVLRPRSPEDVCLMTAIAELHAGGTAIDWAKVLSGGRAVDPLISPHIKWDH SQTWDVPVAEDFPNGSSSSSATVYSIDASPESPDHYLVDHCIDGRVIFPGTGYLCLVWKTLARSLGLSLEETP VVFENVSFHQATILPKTGTVALEVRLLEASHAFEVSDTGNLIVSGKVYLWEDPNSKLFDHPEVPTPPESASV SRLTQGEVYKELRLRGYDYGPQFQGICEATLEGEQGKLLWKDNWVTFMDTMLQVSILGSSQQSLQLPTRV TAIYIDPATHRQKVYRLKEDTQVADVTTSRCLGITVSGGIHISRLQTTATSRRQQEQLVPTLEKFVFTPHMEA ECLSESTALQKELQLCKGLARALQTKATQQGLKAAMLGQEDPPQHGLPRLLAAACQLQLNGNLQLELGEA LAQERLLLPEDPLISGLLNSQALKACVDTALENLSTLKMKVAEVLAGEGHLYSRIPALLNTQPMLQLEYTATD RHPQALKDVQTKLQQHDVAQGQWNPSDPAPSSLGALDLLVCNCALATLGDPALALDNMVAALKEGGFLL VHTVLKGHALGETLACLPSEVQPAPSLLSQEEWESLFSRKALHLVGLKRSFYGTALFLCRRAIPQEKPIFLSVE DTSFQWVDSLKSTLATSSSQPVWLTAMDCPTSGVVGLVNCLRKEPGGHRIRCILLSNLSNTSHAPKLDPGSP ELQQVLKHDLVMNVYRDGAWGAFRHFQLEQDKPKEQTAHAFVNVLTRGDLASIRWVSSPLKHTQPSSSGA QLCTVYYASLNFRDIMLATGKLSPDAIPGKWASRDCMLGMEFSGRDRCGRRVMGLVPAEGLATSVLLSSDF LWDVPSSWTLEEAASVPVVYTTAYYSLVVRGRIQRGETVLIHSGSGGVGQAAISIALSLGCRVFTTVGSAEKR AYLQARFPQLDDTSFANSRDTSFEQHVLLHTGGKGVDLVLNSLAEEKLQASVRCLAQHGRFLEIGKFDLSNN HPLGMAIFLKNVTFHGILLDALFEEANDSWREVAALLKAGIRDGVVKPLKCTVFPKAQVEDAFRYMAQGKH IGKVLVQVREEEPEAVLPGAQPTLISAISKTFCPAHKSYIITGGLGGFGLELARWLVLRGAQRLVLTSRSGIRTG YQAKHIREWRRQGIQVLVSTSNVSSLEGARALIAEATKLGPVGGVFNLAMVLRDAMLENQTPELFQDVNKP KYNGTLNLDRATREACPELDYFVAFSSVSCGRGNAGQTNYGFANSTMERICEQRRHDGLPGLAVQWGAIG DVGIVLEAMGTNDTVIGGTLPQRISSCMEVLDLFLNQPHAVLSSFVLAEKKAVAHGDGDTQRDLVKAVAHIL GIRDLAGINLDSTLADLGLDSLMGVEVRQILEREHDLVLPMREVRQLTLRKLQEMSSKTDSATDTTAPKSRS DTSLKQNQLNLSTLLVNPEGPTLTQLNSVQSSERPLFLVHPIEGSTTVFHSLAAKLSVPTYGLQCTQAAPLD SIPNLAAYYIDCIKQVQPEGPYRIAGYSFGACVAFEMCSQLQAQQGPAPTHNNLFLFDGSHTYVLAYTQSYR AKMTPGCEAEAEAEALCFFIKQFLDVEHSKVLEALLPLKSLEDRVAASVDLITKSHHSLDRRELSFAAVSFYH KLRAADQYKPKAKYHGNVTLLRAKTGGTYGEDLGADYNLSQVCDGKVSVHIIEGDHRTLLEGSGLESIINII HSSLAEPRVSVREGLEHHHHHHHH 
FS037 Strepl-m(KS-LD)-DEBS3(AT)5-H8

MSAWSHPQFEKGGGSGGGSGGSAWSHPQFEKGAGSEEVVIAGMSGKLPESENLQEFWANLIGGVDMVTDDD RRWKAGLYGLPKRSGKLKDLSKFDASFFGVHPKQAHTMDPQLRLLLEVSYEAIVDGGINPASLRGTNTGVW VGVSGSEASEALSRDPETLLGYSMVGCQRAMMANRLSFFFDFKGPSIALDTACSSSLLALQNAYQAIRSGEC PAALVGGINLLLKPNTSVQFMKLGMLSPDGTCRSFDDSGSGYCRSEAVVAVLLTKKSLARRVYATILNAGTN TDGSKEQGVTFPSGEVQEQLICSLYQPAGLAPESLEYIEAHGTGTKVGDPQELNGITRSLCAFRQAPLLIGST KSNMGHPEPASGLAALTKVLLSLEHGVWAPNLHFHNPNPEIPALLDGRLQVVDRPLPVRGGNVGINSFGFGG SNVHVILQPNTRQAPAPTAHAALPHLLHASGRTLEAVQDLLEQGRQHSQDLAFVSMLNDIAATPTAAMPFR GYTVLGVEGRVQEVQQVSTRRGVAMVFPGQGAQWQGMARDLLRESQVFADSIRDCERALAPHVDW SLTDLLSGARPLDRVDVVQPALFAVMVSLAALWRSHGVEPAAVVGHSQGEIAAAHVAGALTLEDAA KLVAVRSRVLRRLGGQGGMASFGLGTEQAAERIGRFAGALSIASVNGPRSVVVAGESGPLDELIAEC EAEGITARRIPVDYASHSPQVESLREELLTELAGISPVSADVALYSTTTGQPIDTATMDTAYWYANL REQVRFQDATRQLAEAGFDAFVEVSPHPVLTVGIEATLDSALPADAGACVVGTLRRDRGGLADFH TALGEAYAQGINVNPNALFPPVEFPAPRGTPLISPHIKWDHSQTWDVPVAEDFPNGSGSPSAHHHHHHHH

FS038 Strepl-m(KS)-DEBS3(LD-AT)5-H8

MSAWSHPQFEKGGGSGGGSGGSAWSHPQFEKGAGSEEVVIAGMSGKLPESENLQEFWANLIGGVDMVTDDD RRWKAGLYGLPKRSGKLKDLSKFDASFFGVHPKQAHTMDPQLRLLLEVSYEAIVDGGINPASLRGTNTGVW VGVSGSEASEALSRDPETLLGYSMVGCQRAMMANRLSFFFDFKGPSIALDTACSSSLLALQNAYQAIRSGEC PAALVGGINLLLKPNTSVQFMKLGMLSPDGTCRSFDDSGSGYCRSEAVVAVLLTKKSLARRVYATILNAGTN TDGSKEQGVTFPSGEVQEQLICSLYQPAGLAPESLEYIEAHGTGTKVGDPQELNGITRSLCAFRQAPLLIGST KSNMGHPEPASGLAALTKVLLSLEHGVWAPNLHFHNPNPEIPALLDGRLQVVDRPLPVRGGNVGINSFGFGG SNVHVILQPNEADEPEPAPDSGPVPLVLSGRDEQAMRAQAGRLADHLAREPRNSLRDTGFTLATRR SAWEHRAVVVGDRDDALAGLRAVADGRIADRTATGQARTRRGVAMVFPGQGAQWQGMARDLLR ESQVFADSIRDCERALAPHVDWSLTDLLSGARPLDRVDVVQPALFAVMVSLAALWRSHGVEPAAV VGHSQGEIAAAHVAGALTLEDAAKLVAVRSRVLRRLGGQGGMASFGLGTEQAAERIGRFAGALSIAS VNGPRSVVVAGESGPLDELIAECEAEGITARRIPVDYASHSPQVESLREELLTELAGISPVSADVALYS TTTGQPIDTATMDTAYWYANLREQVRFQDATRQLAEAGFDAFVEVSPHPVLTVGIEATLDSALPA DAGACVVGTLRRDRGGLADFHTALGEAYAQGVEVDWSPAFADARPVEPLISPHIKWDHSQTWDVPV AEDFPNGSGSPSAHHHHHHHH

FS057 Strepl-m(KS-LD)-DEBS3(AT)5-H8

MSAWSHPQFEKGGGSGGGSGGSAWSHPQFEKGAGSEEVVIAGMSGKLPESENLQEFWANLIGGVDMVTDDD RRWKAGLYGLPKRSGKLKDLSKFDASFFGVHPKQAHTMDPQLRLLLEVSYEAIVDGGINPASLRGTNTGVW VGVSGSEASEALSRDPETLLGYSMVGCQRAMMANRLSFFFDFKGPSIALDTACSSSLLALQNAYQAIRSGEC PAALVGGINLLLKPNTSVQFMKLGMLSPDGTCRSFDDSGSGYCRSEAVVAVLLTKKSLARRVYATILNAGTN TDGSKEQGVTFPSGEVQEQLICSLYQPAGLAPESLEYIEAHGTGTKVGDPQELNGITRSLCAFRQAPLLIGST KSNMGHPEPASGLAALTKVLLSLEHGVWAPNLHFHNPNPEIPALLDGRLQVVDRPLPVRGGNVGINSFGFGG SNVHVILQPNTRQAPAPTAHAALPHLLHASGRTLEAVQDLLEQGRQHSQDLAFVSMLNDIAATPTAAMPFR GYTVLGVEGRVQEVQQVSTRRGVAMVFPGQGAQWQGMARDLLRESQVFADSIRDCERALAPHVDW SLTDLLSGARPLDRVDVVQPALFAVMVSLAALWRSHGVEPAAVVGHSQGEIAAAHVAGALTLEDAA KLVAVRSRVLRRLGGQGGMASFGLGTEQAAERIGRFAGALSIASVNGPRSVVVAGESGPLDELIAEC EAEGITARRIPVDYASHSPQVESLREELLTELAGISPVSADVALYSTTTGQPIDTATMDTAYWYANL REQVRFQDATRQLAEAGFDAFVEVSPHPVLTVGIEATLDSALPADAGACVVGTLRRDRGGLADFH TALGEAYAQGVEVDWSPAFADARPVPRGTPLISPHIKWDHSQTWDVPVAEDFPNGSGSPSAHHHHHH $\mathrm{HH}$ 
pFS058 Strepl-m(KS-LD)-DEBS3(AT)5-H8

MSAWSHPQFEKGGGSGGGSGGSAWSHPQFEKGAGSEEVVIAGMSGKLPESENLQEFWANLIGGVDMVTDDD RRWKAGLYGLPKRSGKLKDLSKFDASFFGVHPKQAHTMDPQLRLLLEVSYEAIVDGGINPASLRGTNTGVW VGVSGSEASEALSRDPETLLGYSMVGCQRAMMANRLSFFFDFKGPSIALDTACSSSLLALQNAYQAIRSGEC PAALVGGINLLLKPNTSVQFMKLGMLSPDGTCRSFDDSGSGYCRSEAVVAVLLTKKSLARRVYATILNAGTN TDGSKEQGVTFPSGEVQEQLICSLYQPAGLAPESLEYIEAHGTGTKVGDPQELNGITRSLCAFRQAPLLIGST KSNMGHPEPASGLAALTKVLLSLEHGVWAPNLHFHNPNPEIPALLDGRLQVVDRPLPVRGGNVGINSFGFGG SNVHVILQPNTRQAPAPTAHAALPHLLHASGRTLEAVQDLLEQGRQHSQDLAFVSMLNDIAATPTAAMPFR GYTVLGVEGRVQEVQQVSTNKRPLAMVFPGQGAQWQGMARDLLRESQVFADSIRDCERALAPHVD WSLTDLLSGARPLDRVDVVQPALFAVMVSLAALWRSHGVEPAAVVGHSQGEIAAAHVAGALTLED AAKLVAVRSRVLRRLGGQGGMASFGLGTEQAAERIGRFAGALSIASVNGPRSVVVAGESGPLDELIA ECEAEGITARRIPVDYASHSPQVESLREELLTELAGISPVSADVALYSTTTGQPIDTATMDTAYWYA NLREQVRFQDATRQLAEAGFDAFVEVSPHPVLTVGIEATLDSALPADAGACVVGTLRRDRGGLAD FHTALGEAYAQGINVNPNALFPPVEFPAPRGTPLISPHIKWDHSQTWDVPVAEDFPNGSGSPSAHHHHHH $\mathrm{HH}$

FS059 Strepl-m(KS-LD)-DEBS3(AT)5-H8

MSAWSHPQFEKGGGSGGGSGGSAWSHPQFEKGAGSEEVVIAGMSGKLPESENLQEFWANLIGGVDMVTDDD RRWKAGLYGLPKRSGKLKDLSKFDASFFGVHPKQAHTMDPQLRLLLEVSYEAIVDGGINPASLRGTNTGVW VGVSGSEASEALSRDPETLLGYSMVGCQRAMMANRLSFFFDFKGPSIALDTACSSSLLALQNAYQAIRSGEC PAALVGGINLLLKPNTSVQFMKLGMLSPDGTCRSFDDSGSGYCRSEAVVAVLLTKKSLARRVYATILNAGTN TDGSKEQGVTFPSGEVQEQLICSLYQPAGLAPESLEYIEAHGTGTKVGDPQELNGITRSLCAFRQAPLLIGST KSNMGHPEPASGLAALTKVLLSLEHGVWAPNLHFHNPNPEIPALLDGRLQVVDRPLPVRGGNVGINSFGFGG SNVHVILQPNTRQAPAPTAHAALPHLLHASGRTLEAVQDLLEQGRQHSQDLAFVSMLNDIAATPTAAMPFR GYTVLGVEGRVQEVQQVSTNKRPLAMVFPGQGAQWQGMARDLLRESQVFADSIRDCERALAPHVD WSLTDLLSGARPLDRVDVVQPALFAVMVSLAALWRSHGVEPAAVVGHSQGEIAAAHVAGALTLED AAKLVAVRSRVLRRLGGQGGMASFGLGTEQAAERIGRFAGALSIASVNGPRSVVVAGESGPLDELIA ECEAEGITARRIPVDYASHSPQVESLREELLTELAGISPVSADVALYSTTTGQPIDTATMDTAYWYA NLREQVRFQDATRQLAEAGFDAFVEVSPHPVLTVGIEATLDSALPADAGACVVGTLRRDRGGLAD FHTALGEAYAQGVEVDWSPAFADARPVPRGTPLISPHIKWDHSQTWDVPVAEDFPNGSGSPSAHHHH $\mathrm{HHHH}$ 
FS060 Strepl-m(KS)-DEBS3(LD-AT)5-H8

MSAWSHPQFEKGGGSGGGSGGSAWSHPQFEKGAGSEEVVIAGMSGKLPESENLQEFWANLIGGVDMVTDDD RRWKAGLYGLPKRSGKLKDLSKFDASFFGVHPKQAHTMDPQLRLLLEVSYEAIVDGGINPASLRGTNTGVW VGVSGSEASEALSRDPETLLGYSMVGCQRAMMANRLSFFFDFKGPSIALDTACSSSLLALQNAYQAIRSGEC PAALVGGINLLLKPNTSVQFMKLGMLSPDGTCRSFDDSGSGYCRSEAVVAVLLTKKSLARRVYATILNAGTN TDGSKEQGVTFPSGEVQEQLICSLYQPAGLAPESLEYIEAHGTGTKVGDPQELNGITRSLCAFRQAPLLIGST KSNMGHPEPASGLAALTKVLLSLEHGVWAPNLHFHNPNPEIPALLDGRLQVVDRPLPVRGGNVGINSFGFGG SNVHVILQPNEADEPEPAPDSGPVPLVLSGRDEQAMRAQAGRLADHLAREPRNSLRDTGFTLATRR SAWEHRAVVVGDRDDALAGLRAVADGRIADRTATGQARTRRGVAMVFPGQGAQWQGMARDLLR ESQVFADSIRDCERALAPHVDWSLTDLLSGARPLDRVDVVQPALFAVMVSLAALWRSHGVEPAAV VGHSQGEIAAAHVAGALTLEDAAKLVAVRSRVLRRLGGQGGMASFGLGTEQAAERIGRFAGALSIAS VNGPRSVVVAGESGPLDELIAECEAEGITARRIPVDYASHSPQVESLREELLTELAGISPVSADVALYS TTTGQPIDTATMDTAYWYANLREQVRFQDATRQLAEAGFDAFVEVSPHPVLTVGIEATLDSALPA DAGACVVGTLRRDRGGLADFHTALGEAYAQGVEVDWSPAFADARPVPRGTPLISPHIKWDHSQTWD VPVAEDFPNGSGSPSAHHHHHHHH

FS061 Strepl-m(KS)-DEBS3(LD-AT)5-H8

MSAWSHPQFEKGGGSGGGSGGSAWSHPQFEKGAGSEEVVIAGMSGKLPESENLQEFWANLIGGVDMVTDDD RRWKAGLYGLPKRSGKLKDLSKFDASFFGVHPKQAHTMDPQLRLLLEVSYEAIVDGGINPASLRGTNTGVW VGVSGSEASEALSRDPETLLGYSMVGCQRAMMANRLSFFFDFKGPSIALDTACSSSLLALQNAYQAIRSGEC PAALVGGINLLLKPNTSVQFMKLGMLSPDGTCRSFDDSGSGYCRSEAVVAVLLTKKSLARRVYATILNAGTN TDGSKEQGVTFPSGEVQEQLICSLYQPAGLAPESLEYIEAHGTGTKVGDPQELNGITRSLCAFRQAPLLIGST KSNMGHPEPASGLAALTKVLLSLEHGVWAPNLHFHNPNPEIPALLDGRLQVVDRPLPVRGGNVGINSFGFG GTNAHVIVEEAPEADEPEPAPDSGPVPLVLSGRDEQAMRAQAGRLADHLAREPRNSLRDTGFTLA TRRSAWEHRAVVVGDRDDALAGLRAVADGRIADRTATGQARTRRGVAMVFPGQGAQWQGMAR DLLRESQVFADSIRDCERALAPHVDWSLTDLLSGARPLDRVDVVQPALFAVMVSLAALWRSHGVE PAAVVGHSQGEIAAAHVAGALTLEDAAKLVAVRSRVLRRLGGQGGMASFGLGTEQAAERIGRFAGA LSIASVNGPRSVVVAGESGPLDELIAECEAEGITARRIPVDYASHSPQVESLREELLTELAGISPVSAD VALYSTTTGQPIDTATMDTAYWYANLREQVRFQDATRQLAEAGFDAFVEVSPHPVLTVGIEATLD SALPADAGACVVGTLRRDRGGLADFHTALGEAYAQGVEVDWSPAFADARPVEPLISPHIKWDHSQT WDVPVAEDFPNGSGSPSAHHHHHHHH 
FS062 Strepl-m(KS)-DEBS3(LD-AT)5-H8

MSAWSHPQFEKGGGSGGGSGGSAWSHPQFEKGAGSEEVVIAGMSGKLPESENLQEFWANLIGGVDMVTDDD RRWKAGLYGLPKRSGKLKDLSKFDASFFGVHPKQAHTMDPQLRLLLEVSYEAIVDGGINPASLRGTNTGVW VGVSGSEASEALSRDPETLLGYSMVGCQRAMMANRLSFFFDFKGPSIALDTACSSSLLALQNAYQAIRSGEC PAALVGGINLLLKPNTSVQFMKLGMLSPDGTCRSFDDSGSGYCRSEAVVAVLLTKKSLARRVYATILNAGTN TDGSKEQGVTFPSGEVQEQLICSLYQPAGLAPESLEYIEAHGTGTKVGDPQELNGITRSLCAFRQAPLLIGST KSNMGHPEPASGLAALTKVLLSLEHGVWAPNLHFHNPNPEIPALLDGRLQVVDRPLPVRGGNVGINSFGFG GTNAHVIVEEAPEADEPEPAPDSGPVPLVLSGRDEQAMRAQAGRLADHLAREPRNSLRDTGFTLA TRRSAWEHRAVVVGDRDDALAGLRAVADGRIADRTATGQARTRRGVAMVFPGQGAQWQGMAR DLLRESQVFADSIRDCERALAPHVDWSLTDLLSGARPLDRVDVVQPALFAVMVSLAALWRSHGVE PAAVVGHSQGEIAAAHVAGALTLEDAAKLVAVRSRVLRRLGGQGGMASFGLGTEQAAERIGRFAGA LSIASVNGPRSVVVAGESGPLDELIAECEAEGITARRIPVDYASHSPQVESLREELLTELAGISPVSAD VALYSTTTGQPIDTATMDTAYWYANLREQVRFQDATRQLAEAGFDAFVEVSPHPVLTVGIEATLD SALPADAGACVVGTLRRDRGGLADFHTALGEAYAQGVEVDWSPAFADARPVPRGTPLISPHIKWDH SQTWDVPVAEDFPNGSGSPSAHHHHHHHH

FS116 Strepl-m(KS-LD)-Ecoli(FabD)-H8

MSAWSHPQFEKGGGSGGGSGGSAWSHPQFEKGAGSEEVVIAGMSGKLPESENLQEFWANLIGGVDMVTDDD RRWKAGLYGLPKRSGKLKDLSKFDASFFGVHPKQAHTMDPQLRLLLEVSYEAIVDGGINPASLRGTNTGVW VGVSGSEASEALSRDPETLLGYSMVGCQRAMMANRLSFFFDFKGPSIALDTACSSSLLALQNAYQAIRSGEC PAALVGGINLLLKPNTSVQFMKLGMLSPDGTCRSFDDSGSGYCRSEAVVAVLLTKKSLARRVYATILNAGTN TDGSKEQGVTFPSGEVQEQLICSLYQPAGLAPESLEYIEAHGTGTKVGDPQELNGITRSLCAFRQAPLLIGST KSNMGHPEPASGLAALTKVLLSLEHGVWAPNLHFHNPNPEIPALLDGRLQVVDRPLPVRGGNVGINSFGFGG SNVHVILQPNTRQAPAPTAHAALPHLLHASGRTLEAVQDLLEQGRQHSQDLAFVSMLNDIAATPTAAMPFR GYTVLGVEGRVQEVQQVSTQFAFVFPGQGSQTVGMLADMAASYPIVEETFAEASAALGYDLWALTQ QGPAEELNKTWQTQPALLTASVALYRVWQQQGGKAPAMMAGHSLGEYSALVCAGVIDFADAVRL VEMRGKFMQEAVPEGTGAMAAIIGLDDASIAKACEEAAEGQVVSPVNFNSPGQVVIAGHKEAVER AGAACKAAGAKRALPLPVSVPSHCALMKPAADKLAVELAKITFNAPTVPVVNNVDVKCETNGDA IRDALVRQLYNPVQWTKSVEYMAAQGVEHLYEVGPGKVLTGLTKRIVDTLTASALNEPSAMAAAL

ELGINVNPNALFPPVEFPAPRGTPLISPHIKWDHSQTWDVPVAEDFPNGSGSPSAHHHHHHHH

FS117 Strepl-m(KS-LD)-Ecoli(FabD)-H8

MSAWSHPQFEKGGGSGGGSGGSAWSHPQFEKGAGSEEVVIAGMSGKLPESENLQEFWANLIGGVDMVTDDD RRWKAGLYGLPKRSGKLKDLSKFDASFFGVHPKQAHTMDPQLRLLLEVSYEAIVDGGINPASLRGTNTGVW VGVSGSEASEALSRDPETLLGYSMVGCQRAMMANRLSFFFDFKGPSIALDTACSSSLLALQNAYQAIRSGEC PAALVGGINLLLKPNTSVQFMKLGMLSPDGTCRSFDDSGSGYCRSEAVVAVLLTKKSLARRVYATILNAGTN TDGSKEQGVTFPSGEVQEQLICSLYQPAGLAPESLEYIEAHGTGTKVGDPQELNGITRSLCAFRQAPLLIGST KSNMGHPEPASGLAALTKVLLSLEHGVWAPNLHFHNPNPEIPALLDGRLQVVDRPLPVRGGNVGINSFGFGG SNVHVILQPNTRQAPAPTAHAALPHLLHASGRTLEAVQDLLEQGRQHSQDLAFVSMLNDIAATPTAAMPFR GYTVLGVEGRVQEVQQVSTNKTQFAFVFPGQGSQTVGMLADMAASYPIVEETFAEASAALGYDLWA LTQQGPAEELNKTWQTQPALLTASVALYRVWQQQGGKAPAMMAGHSLGEYSALVCAGVIDFADA VRLVEMRGKFMQEAVPEGTGAMAAIIGLDDASIAKACEEAAEGQVVSPVNFNSPGQVVIAGHKEA VERAGAACKAAGAKRALPLPVSVPSHCALMKPAADKLAVELAKITFNAPTVPVVNNVDVKCETN GDAIRDALVRQLYNPVQWTKSVEYMAAQGVEHLYEVGPGKVLTGLTKRIVDTLTASALNEPSAMA AALELGINVNPNALFPPVEFPAPRGTPLISPHIKWDHSQTWDVPVAEDFPNGSGSPSAHHHHHHHH 
FS118 Strepl-m(KS-LD)-DEBS3(AT)6-H8

MSAWSHPQFEKGGGSGGGSGGSAWSHPQFEKGAGSEEVVIAGMSGKLPESENLQEFWANLIGGVDMVTDDD RRWKAGLYGLPKRSGKLKDLSKFDASFFGVHPKQAHTMDPQLRLLLEVSYEAIVDGGINPASLRGTNTGVW VGVSGSEASEALSRDPETLLGYSMVGCQRAMMANRLSFFFDFKGPSIALDTACSSSLLALQNAYQAIRSGEC PAALVGGINLLLKPNTSVQFMKLGMLSPDGTCRSFDDSGSGYCRSEAVVAVLLTKKSLARRVYATILNAGTN TDGSKEQGVTFPSGEVQEQLICSLYQPAGLAPESLEYIEAHGTGTKVGDPQELNGITRSLCAFRQAPLLIGST KSNMGHPEPASGLAALTKVLLSLEHGVWAPNLHFHNPNPEIPALLDGRLQVVDRPLPVRGGNVGINSFGFGG SNVHVILQPNTRQAPAPTAHAALPHLLHASGRTLEAVQDLLEQGRQHSQDLAFVSMLNDIAATPTAAMPFR GYTVLGVEGRVQEVQQVSTAGGVVFVFPGQGAQWEGMARGLLSVPVFAESIAECDAVLSEVAGFSAS EVLEQRPDAPSLERVDVVQPVLFSVMVSLARLWGACGVSPSAVIGHSQGEIAAAVVAGVLSLEDGV RVVALRAKALRALAGKGGMVSLAAPGERARALIAPWEDRISVAAVNSPSSVVVSGDPEALAELVAR CEDEGVRAKTLPVDYASHSRHVEEIRETILADLDGISARRAAIPLYSTLHGERRDGADMGPRYWY DNLRSQVRFDEAVSAAVADGHATFVEMSPHPVLTAAVQEIAADAVAIGSLHRDTAEEHLIAELARA HVHGINVNPNALFPPVEFPAPRGTPLISPHIKWDHSQTWDVPVAEDFPNGSGSPSAHHHHHHHH

FS119 Strepl-m(KS-LD)-DEBS3(AT)6-H8

MSAWSHPQFEKGGGSGGGSGGSAWSHPQFEKGAGSEEVVIAGMSGKLPESENLQEFWANLIGGVDMVTDDD RRWKAGLYGLPKRSGKLKDLSKFDASFFGVHPKQAHTMDPQLRLLLEVSYEAIVDGGINPASLRGTNTGVW VGVSGSEASEALSRDPETLLGYSMVGCQRAMMANRLSFFFDFKGPSIALDTACSSSLLALQNAYQAIRSGEC PAALVGGINLLLKPNTSVQFMKLGMLSPDGTCRSFDDSGSGYCRSEAVVAVLLTKKSLARRVYATILNAGTN TDGSKEQGVTFPSGEVQEQLICSLYQPAGLAPESLEYIEAHGTGTKVGDPQELNGITRSLCAFRQAPLLIGST KSNMGHPEPASGLAALTKVLLSLEHGVWAPNLHFHNPNPEIPALLDGRLQVVDRPLPVRGGNVGINSFGFGG SNVHVILQPNTRQAPAPTAHAALPHLLHASGRTLEAVQDLLEQGRQHSQDLAFVSMLNDIAATPTAAMPFR GYTVLGVEGRVQEVQQVSAGGVVFVFPGQGAQWEGMARGLLSVPVFAESIAECDAVLSEVAGFSASE VLEQRPDAPSLERVDVVQPVLFSVMVSLARLWGACGVSPSAVIGHSQGEIAAAVVAGVLSLEDGVR VVALRAKALRALAGKGGMVSLAAPGERARALIAPWEDRISVAAVNSPSSVVVSGDPEALAELVARC EDEGVRAKTLPVDYASHSRHVEEIRETILADLDGISARRAAIPLYSTLHGERRDGADMGPRYWYD NLRSQVRFDEAVSAAVADGHATFVEMSPHPVLTAAVQEIAADAVAIGSLHRDTAEEHLIAELARAH VHGINVNPNALFPPVEFPAPRGTPLISPHIKWDHSQTWDVPVAEDFPNGSGSPSAHHHHHHHH

FS139 Strepl-DEBS3(KS-LD)6-m(AT)-H8

MSAWSHPQFEKGGGSGGGSGGSAWSHPQFEKGAGSPIAIVGMACRFPGGVHNPGELWEFIVGGGDAVTEMP TDRGWDLDALFDPDPQRHGTSYSRHGAFLDGAADFDAAFFGISPREALAMDPQQRQVLETTWELFENAGI DPHSLRGSDTGVFLGAAYQGYGQDAVVPEDSEGYLLTGNSSAVVSGRVAYVLGLEGPAVTVDTACSSSLVAL HSACGSLRDGDCGLAVAGGVSVMAGPEVFTEFSRQGGLAVDGRCKAFSAEADGFGFAEGVAVVLLQRLSDA RRAGRQVLGVVAGSAINQDGASNGLAAPSGVAQQRVIRKAWARAGITGADVAVVEAHGTGTRLGDPVEASA LLATYGKSRGSSGPVLLGSVKSNIGHAQAAAGVAGVIKVVLGLNRGLVPPMLCRGERSPLIEWSSGGVELAEA VSPWPPAADGVRRAGVSAFGVSGTNAHVIIAEPPEPEPLPEPGPVGVLAAANSVPVLLSARTETALAAQARL LESAVDDSVPLTALASALATGRAHLPRRAALLAGDHEQLRGQLRAVAEGVAAPGATTGTASNKRPLWFICS GMGTQWRGMGLSLMRLDSFRESILRSDEAVKPLGVKVSDLLLSTDERTFDDIVHAFVSLTAIQIALI DLLTSVGLKPDGIIGHSLGEVACGYADGCLSQREAVLAAYWRGQCIKDAHLPPGSMAAVGLSWEE CKQRCPAGVVPACHNSEDTVTISGPQAAVNEFVEQLKQEGVFAKEVRTGGLAFHSYFMEGIAPTL LQALKKVIREPRPRSARWLSTSIPEAQWQSSLARTSSAEYNVNNLVSPVLFQEALWHIPEHAVVLEI APHALLQAVLKRGVKSSCTIIPLMKRDHKDNLEFFLTNLGKVHLTGVAVDWRNVFPAAPPVALPNYPF EPQRYWLAPEVSDQLADSLEHHHHHHHH 
FS163 Strepl-DEBS(DD)5-m(KS-LD)-VemG(AT-ACP)-SZ3-H6

MSAWSHPQFEKGGGSGGGSGGSAWSHPQFEKGAGSSGDNGMTEEKLRRYLKRTVTELDSVTARLREVEHR AGEEVVIAGMSGKLPESENLQEFWANLIGGVDMVTDDDRRWKAGLYGLPKRSGKLKDLSKFDASFFGVHP KQAHTMDPQLRLLLEVSYEAIVDGGINPASLRGTNTGVWVGVSGSEASEALSRDPETLLGYSMVGCQRAM MANRLSFFFDFKGPSIALDTACSSSLLALQNAYQAIRSGECPAALVGGINLLLKPNTSVQFMKLGMLSPDGTC RSFDDSGSGYCRSEAVVAVLLTKKSLARRVYATILNAGTNTDGSKEQGVTFPSGEVQEQLICSLYQPAGLAPE SLEYIEAHGTGTKVGDPQELNGITRSLCAFRQAPLLIGSTKSNMGHPEPASGLAALTKVLLSLEHGVWAPNL HFHNPNPEIPALLDGRLQVVDRPLPVRGGNVGINSFGFGGSNVHVILQPNTRQAPAPTAHAALPHLLHASGR TLEAVQDLLEQGRQHSQDLAFVSMLNDIAATPTAAMPFRGYTVLGVEGRVQEVQQVSTEPSLAIGFTGQG SQHPGMGRELYAAFPVFAEALDAAWAALDPHLERPLRDVMWAPDGTARAALLDRTDFTQAALFA LEGALYRLVESWGVVPDVVLGHSVGALTAAHAAGVLSLPDAASLVAARGRLMAALPPGGAMTSIE ATEDELRTELRATDGVLAIAAVNGPRSVVVSGEEKAVRTVGEAFRARGRRVTALRVSHAFHSPLM DPMVEEFRAAAARVTYRPPLLPMISDLTGRPADPAHLRSPDHWVRHVRETVRFADAVRALPGQG VTAFLELGPDRQLTTMAAAGAPGSGPALCGGLRRGRSEVRSLLDAVAQAHVRGVPVDWREFFAGG PTRPVDLPVYPFEHRRYWLASAPTRTGGATSVPEAPAPPTGDTPGPDRLRADLAPLTDDERERR LTDLVRGEIAAVAGFDGPHEIEAHRSMTDLGLDSVSAVDLSTRLGARTGLDLPASLAFDHPTSTAIA RHLLAALGGGSGGGSGNEVTTLENDAAFIENENAYLEKEIARLRKEKAALRNRLAHKKLEHHHHHH

FS164 Strepl-DEBS(DD)5-m(KS)-VemG(LD-AT-ACP)-SZ3-H6

MSAWSHPQFEKGGGSGGGSGGSAWSHPQFEKGAGSSGDNGMTEEKLRRYLKRTVTELDSVTARLREVEHR AGEEVVIAGMSGKLPESENLQEFWANLIGGVDMVTDDDRRWKAGLYGLPKRSGKLKDLSKFDASFFGVHP KQAHTMDPQLRLLLEVSYEAIVDGGINPASLRGTNTGVWVGVSGSEASEALSRDPETLLGYSMVGCQRAM MANRLSFFFDFKGPSIALDTACSSSLLALQNAYQAIRSGECPAALVGGINLLLKPNTSVQFMKLGMLSPDGTC RSFDDSGSGYCRSEAVVAVLLTKKSLARRVYATILNAGTNTDGSKEQGVTFPSGEVQEQLICSLYQPAGLAPE SLEYIEAHGTGTKVGDPQELNGITRSLCAFRQAPLLIGSTKSNMGHPEPASGLAALTKVLLSLEHGVWAPNL HFHNPNPEIPALLDGRLQVVDRPLPVRGGNVGINSFGFGGSNVHVILQPNEPDAPPALPPAVHPDTMPVL LSARTPSALRRQAERVLDAVRADPGLPVPDLAAALATTRTAFPRRAAFVVEGRAELERRLRSFAD GEDGGAEPDEVPAEPSLAIGFTGQGSQHPGMGRELYAAFPVFAEALDAAWAALDPHLERPLRDV MWAPDGTARAALLDRTDFTQAALFALEGALYRLVESWGVVPDVVLGHSVGALTAAHAAGVLSLP DAASLVAARGRLMAALPPGGAMTSIEATEDELRTELRATDGVLAIAAVNGPRSVVVSGEEKAVRT VGEAFRARGRRVTALRVSHAFHSPLMDPMVEEFRAAAARVTYRPPLLPMISDLTGRPADPAHLR SPDHWVRHVRETVRFADAVRALPGQGVTAFLELGPDRQLTTMAAAGAPGSGPALCGGLRRGRSE VRSLLDAVAQAHVRGVPVDWREFFAGGPTRPVDLPVYPFEHRRYWLASAPTRTGGATSVPEAPA PPTGDTPGPDRLRADLAPLTDDERERRLTDLVRGEIAAVAGFDGPHEIEAHRSMTDLGLDSVSAVD LSTRLGARTGLDLPASLAFDHPTSTAIARHLLAALGGGSGGGSGNEVTTLENDAAFIENENAYLEKEIAR LRKEKAALRNRLAHKKLEHHHHHH 
FS165 Strepl-DEBS(DD)5-m(KS-LD)-VemH(AT-ACP)-SZ3-H6b

MSAWSHPQFEKGGGSGGGSGGSAWSHPQFEKGAGSSGDNGMTEEKLRRYLKRTVTELDSVTARLREVEHR AGEEVVIAGMSGKLPESENLQEFWANLIGGVDMVTDDDRRWKAGLYGLPKRSGKLKDLSKFDASFFGVHP KQAHTMDPQLRLLLEVSYEAIVDGGINPASLRGTNTGVWVGVSGSEASEALSRDPETLLGYSMVGCQRAM MANRLSFFFDFKGPSIALDTACSSSLLALQNAYQAIRSGECPAALVGGINLLLKPNTSVQFMKLGMLSPDGTC RSFDDSGSGYCRSEAVVAVLLTKKSLARRVYATILNAGTNTDGSKEQGVTFPSGEVQEQLICSLYQPAGLAPE SLEYIEAHGTGTKVGDPQELNGITRSLCAFRQAPLLIGSTKSNMGHPEPASGLAALTKVLLSLEHGVWAPNL HFHNPNPEIPALLDGRLQVVDRPLPVRGGNVGINSFGFGGSNVHVILQPNTRQAPAPTAHAALPHLLHASGR TLEAVQDLLEQGRQHSQDLAFVSMLNDIAATPTAAMPFRGYTVLGVEGRVQEVQQVSTPGGTALLFSGQ GSQRVGMGSELYETYPVFAESFDAVAEHTGLPLKDVVLGGTPDGLLDRTRYAQPALFAVEVSLFRL VRALGLDVRAVVGHSVGEIAAAHVAGVMSMADACRLVEARGRLMDALPPGGAMVAVEVTEAEAS AALAGLEDRVAVAAVNGPASTVLSGEEGAVLKLADAWRERGVRTHRLTVSHAFHSPLMEPMVDA FREVVAGLDLHRPTLAGLPAEVVDPEYWVRHVRRPVRFADAVARAREAGAVRWLEVGPGGVLTA LAQRIVPDTEEHVFAAALRTDRPEPEALLVALSQVHVDGGTVDWSGLCAGGRLVDLPTYPFQRQH YWIEDQPLPPTAPRPGTAPSGTGTAAEGAAAAEVPLSERLARLTGAERLAAVRELVLAEASETLGH TGTLITADRTRQELGFDSLTAIELRNRISRTLGVRLPPTLVFDHEDLGEIASFVDARLGGGSGGGSGNE VTTLENDAAFIENENAYLEKEIARLRKEKAALRNRLAHKKLEHHHHHH

FS166 Strepl-DEBS(DD)5-m(KS)-VemH(LD-AT-ACP)-SZ3H6

MSAWSHPQFEKGGGSGGGSGGSAWSHPQFEKGAGSSGDNGMTEEKLRRYLKRTVTELDSVTARLREVEHR AGEEVVIAGMSGKLPESENLQEFWANLIGGVDMVTDDDRRWKAGLYGLPKRSGKLKDLSKFDASFFGVHP KQAHTMDPQLRLLLEVSYEAIVDGGINPASLRGTNTGVWVGVSGSEASEALSRDPETLLGYSMVGCQRAM MANRLSFFFDFKGPSIALDTACSSSLLALQNAYQAIRSGECPAALVGGINLLLKPNTSVQFMKLGMLSPDGTC RSFDDSGSGYCRSEAVVAVLLTKKSLARRVYATILNAGTNTDGSKEQGVTFPSGEVQEQLICSLYQPAGLAPE SLEYIEAHGTGTKVGDPQELNGITRSLCAFRQAPLLIGSTKSNMGHPEPASGLAALTKVLLSLEHGVWAPNL HFHNPNPEIPALLDGRLQVVDRPLPVRGGNVGINSFGFGGSNVHVILQPNEEPARDAAETRPEARSREAA EGRREAAGQGRTETGPGPSATPPEAPGRARPPVPWPLSGRDAGALRDQIGRLRAHLDAAPADPE DVAHSLARRAVFRHRAVLLAAPQAPAGGSPRAVTGVARPGGTALLFSGQGSQRVGMGSELYETYP VFAESFDAVAEHTGLPLKDVVLGGTPDGLLDRTRYAQPALFAVEVSLFRLVRALGLDVRAVVGHSV GEIAAAHVAGVMSMADACRLVEARGRLMDALPPGGAMVAVEVTEAEASAALAGLEDRVAVAAVN GPASTVLSGEEGAVLKLADAWRERGVRTHRLTVSHAFHSPLMEPMVDAFREVVAGLDLHRPTLA GLPAEVVDPEYWVRHVRRPVRFADAVARAREAGAVRWLEVGPGGVLTALAQRIVPDTEEHVFAAA LRTDRPEPEALLVALSQVHVDGGTVDWSGLCAGGRLVDLPTYPFQRQHYWIEDQPLPPTAPRPG TAPSGTGTAAEGAAAAEVPLSERLARLTGAERLAAVRELVLAEASETLGHTGTLITADRTRQELGFD SLTAIELRNRISRTLGVRLPPTLVFDHEDLGEIASFVDARLGGGSGGGSGNEVTTLENDAAFIENENAYL EKEIARLRKEKAALRNRLAHKKLEHHHHHH 
FS167 Strepl-DEBS(DD)5-m(KS-LD)-DEBS3(AT)6-m(L-ACP)-SZ3-H6

MSAWSHPQFEKGGGSGGGSGGSAWSHPQFEKGAGSSGDNGMTEEKLRRYLKRTVTELDSVTARLREVEHR AGEEVVIAGMSGKLPESENLQEFWANLIGGVDMVTDDDRRWKAGLYGLPKRSGKLKDLSKFDASFFGVHP KQAHTMDPQLRLLLEVSYEAIVDGGINPASLRGTNTGVWVGVSGSEASEALSRDPETLLGYSMVGCQRAM MANRLSFFFDFKGPSIALDTACSSSLLALQNAYQAIRSGECPAALVGGINLLLKPNTSVQFMKLGMLSPDGTC RSFDDSGSGYCRSEAVVAVLLTKKSLARRVYATILNAGTNTDGSKEQGVTFPSGEVQEQLICSLYQPAGLAPE SLEYIEAHGTGTKVGDPQELNGITRSLCAFRQAPLLIGSTKSNMGHPEPASGLAALTKVLLSLEHGVWAPNL HFHNPNPEIPALLDGRLQVVDRPLPVRGGNVGINSFGFGGSNVHVILQPNTRQAPAPTAHAALPHLLHASGR TLEAVQDLLEQGRQHSQDLAFVSMLNDIAATPTAAMPFRGYTVLGVEGRVQEVQQVSTAGGVVFVFPGQ GAQWEGMARGLLSVPVFAESIAECDAVLSEVAGFSASEVLEQRPDAPSLERVDVVQPVLFSVMVSL ARLWGACGVSPSAVIGHSQGEIAAAVVAGVLSLEDGVRVVALRAKALRALAGKGGMVSLAAPGERA RALIAPWEDRISVAAVNSPSSVVVSGDPEALAELVARCEDEGVRAKTLPVDYASHSRHVEEIRETIL ADLDGISARRAAIPLYSTLHGERRDGADMGPRYWYDNLRSQVRFDEAVSAAVADGHATFVEMSP HPVLTAAVQEIAADAVAIGSLHRDTAEEHLIAELARAHVHGINVNPNALFPPVEFPAPRGTPLISPHIKW DHSQTWDVPVAEDFPNGSSSSSATVYSIDASAEKKAVAHGDGDTQRDLVKAVAHILGIRDLAGINLDSTLAD LGLDSLMGVEVRQILEREHDLVLPMREVRQLTLRKLQEMSSKTDSATDTTGGGSGGGSGNEVTTLENDAAF IENENAYLEKEIARLRKEKAALRNRLAHKKLEHHHHHH

FS168 Strepl-DEBS(DD)5-m(KS-LD)-DEBS3(AT)6-m(L)-DEBS3(ACP)6-SZ3-H6 MSAWSHPQFEKGGGSGGGSGGSAWSHPQFEKGAGSSGDNGMTEEKLRRYLKRTVTELDSVTARLREVEHR AGEEVVIAGMSGKLPESENLQEFWANLIGGVDMVTDDDRRWKAGLYGLPKRSGKLKDLSKFDASFFGVHP KQAHTMDPQLRLLLEVSYEAIVDGGINPASLRGTNTGVWVGVSGSEASEALSRDPETLLGYSMVGCQRAM MANRLSFFFDFKGPSIALDTACSSSLLALQNAYQAIRSGECPAALVGGINLLLKPNTSVQFMKLGMLSPDGTC RSFDDSGSGYCRSEAVVAVLLTKKSLARRVYATILNAGTNTDGSKEQGVTFPSGEVQEQLICSLYQPAGLAPE SLEYIEAHGTGTKVGDPQELNGITRSLCAFRQAPLLIGSTKSNMGHPEPASGLAALTKVLLSLEHGVWAPNL HFHNPNPEIPALLDGRLQVVDRPLPVRGGNVGINSFGFGGSNVHVILQPNTRQAPAPTAHAALPHLLHASGR TLEAVQDLLEQGRQHSQDLAFVSMLNDIAATPTAAMPFRGYTVLGVEGRVQEVQQVSTAGGVVFVFPGQ GAQWEGMARGLLSVPVFAESIAECDAVLSEVAGFSASEVLEQRPDAPSLERVDVVQPVLFSVMVSL ARLWGACGVSPSAVIGHSQGEIAAAVVAGVLSLEDGVRVVALRAKALRALAGKGGMVSLAAPGERA RALIAPWEDRISVAAVNSPSSVVVSGDPEALAELVARCEDEGVRAKTLPVDYASHSRHVEEIRETIL ADLDGISARRAAIPLYSTLHGERRDGADMGPRYWYDNLRSQVRFDEAVSAAVADGHATFVEMSP HPVLTAAVQEIAADAVAIGSLHRDTAEEHLIAELARAHVHGINVNPNALFPPVEFPAPRGTPLISPHIKW DHSQTWDVPVAEDFPNGSSSSSATVYSIDASAEKKAVAAPAREMTSQELLEFTHSHVAAILGHSSPDAV GQDQPFTELGFDSLTAVGLRNQLQQATGLALPATLVFEHPTVRRLADHIGQQLDTTGGGSGGGSGN EVTTLENDAAFIENENAYLEKEIARLRKEKAALRNRLAHKKLEHHHHHH 
FS170 Strepl-DEBS(DD)5-m(KS-LD)-EcPKS1(AT-L-ACP)-SZ3-H6

MSAWSHPQFEKGGGSGGGSGGSAWSHPQFEKGAGSSGDNGMTEEKLRRYLKRTVTELDSVTARLREVEHR AGEEVVIAGMSGKLPESENLQEFWANLIGGVDMVTDDDRRWKAGLYGLPKRSGKLKDLSKFDASFFGVHP KQAHTMDPQLRLLLEVSYEAIVDGGINPASLRGTNTGVWVGVSGSEASEALSRDPETLLGYSMVGCQRAM MANRLSFFFDFKGPSIALDTACSSSLLALQNAYQAIRSGECPAALVGGINLLLKPNTSVQFMKLGMLSPDGTC RSFDDSGSGYCRSEAVVAVLLTKKSLARRVYATILNAGTNTDGSKEQGVTFPSGEVQEQLICSLYQPAGLAPE SLEYIEAHGTGTKVGDPQELNGITRSLCAFRQAPLLIGSTKSNMGHPEPASGLAALTKVLLSLEHGVWAPNL HFHNPNPEIPALLDGRLQVVDRPLPVRGGNVGINSFGFGGSNVHVILQPNTRQAPAPTAHAALPHLLHASGR TLEAVQDLLEQGRQHSQDLAFVSMLNDIAATPTAAMPFRGYTVLGVEGRVQEVQQVSTAREVWFIYAGM GSQWVGMARCLMQLDVFRHSLEKSAAVLKPHGVDLLNILSEGTEDTLRTILNPFVCITSIQVALTD LLWSMGIRPDGIVGHSMGEVGCAYCDGCLTAEEAVLTAYWRGKCVTDGKVPPGKMAAVGLTWEE AKSQCPAGVVPACHNAEDSVTISGAADVMLKFMEELKAKDVFVREVNSSNIAYHSYFMENIYASL KDSLSKVISPKPRTARWLPTSVPEELWDSAPAQSSSAEFHANNLVSPVLFHEALQKIPPTAIAIELA PHGLLQSVIKRTLGNESVCVGLQKRNYADNLEFLFASLGKCFANGLSLNPLACYPPVEFPVPKGTP RLSDMVAGAWDHSAQWLVPKNEDFEGRVQASGSDSSYSIDVSAEKVKAAVEGEETVGQQVIKAV GNVLGLKSVSGVDPDKVFLDLGLDSLMSVEIKQMLERDLDLALGTKDIQMLTFAQLQAMVGGGSG GGSGNEVTTLENDAAFIENENAYLEKEIARLRKEKAALRNRLAHKKLEHHHHHH

FS171 Strepl-DEBS(DD)5-m(KS)-EcPKS1(LD-AT-L-ACP)-SZ3-H6

MSAWSHPQFEKGGGSGGGSGGSAWSHPQFEKGAGSSGDNGMTEEKLRRYLKRTVTELDSVTARLREVEHR AGEEVVIAGMSGKLPESENLQEFWANLIGGVDMVTDDDRRWKAGLYGLPKRSGKLKDLSKFDASFFGVHP KQAHTMDPQLRLLLEVSYEAIVDGGINPASLRGTNTGVWVGVSGSEASEALSRDPETLLGYSMVGCQRAM MANRLSFFFDFKGPSIALDTACSSSLLALQNAYQAIRSGECPAALVGGINLLLKPNTSVQFMKLGMLSPDGTC RSFDDSGSGYCRSEAVVAVLLTKKSLARRVYATILNAGTNTDGSKEQGVTFPSGEVQEQLICSLYQPAGLAPE SLEYIEAHGTGTKVGDPQELNGITRSLCAFRQAPLLIGSTKSNMGHPEPASGLAALTKVLLSLEHGVWAPNL HFHNPNPEIPALLDGRLQVVDRPLPVRGGNVGINSFGFGGSNVHVILQPNVSSSKPHPSAHKPRLFTYSA RTEHGLRAILREAQTHAASMEFHALCQASADAPLGSMPYRGATILNGQHDFEVVEKCKSKAREV WFIYAGMGSQWVGMARCLMQLDVFRHSLEKSAAVLKPHGVDLLNILSEGTEDTLRTILNPFVCIT SIQVALTDLLWSMGIRPDGIVGHSMGEVGCAYCDGCLTAEEAVLTAYWRGKCVTDGKVPPGKMA AVGLTWEEAKSQCPAGVVPACHNAEDSVTISGAADVMLKFMEELKAKDVFVREVNSSNIAYHSYF MENIYASLKDSLSKVISPKPRTARWLPTSVPEELWDSAPAQSSSAEFHANNLVSPVLFHEALQKIPP TAIAIELAPHGLLQSVIKRTLGNESVCVGLQKRNYADNLEFLFASLGKCFANGLSLNPLACYPPVEFP VPKGTPRLSDMVAGAWDHSAQWLVPKNEDFEGRVQASGSDSSYSIDVSAEKVKAAVEGEETVGQ QVIKAVGNVLGLKSVSGVDPDKVFLDLGLDSLMSVEIKQMLERDLDLALGTKDIQMLTFAQLQAM VGGGSGGGSGNEVTTLENDAAFIENENAYLEKEIARLRKEKAALRNRLAHKKLEHHHHHH 


\section{Appendix}

FS172 Strepl-DEBS(DD)5-m(KS)-EcPKS1(LD-AT-L-ACP)-SZ3-H6

MSAWSHPQFEKGGGSGGGSGGSAWSHPQFEKGAGSSGDNGMTEEKLRRYLKRTVTELDSVTARLREVEHR AGEEVVIAGMSGKLPESENLQEFWANLIGGVDMVTDDDRRWKAGLYGLPKRSGKLKDLSKFDASFFGVHP KQAHTMDPQLRLLLEVSYEAIVDGGINPASLRGTNTGVWVGVSGSEASEALSRDPETLLGYSMVGCQRAM MANRLSFFFDFKGPSIALDTACSSSLLALQNAYQAIRSGECPAALVGGINLLLKPNTSVQFMKLGMLSPDGTC RSFDDSGSGYCRSEAVVAVLLTKKSLARRVYATILNAGTNTDGSKEQGVTFPSGEVQEQLICSLYQPAGLAPE SLEYIEAHGTGTKVGDPQELNGITRSLCAFRQAPLLIGSTKSNMGHPEPASGLAALTKVLLSLEHGVWAPNL HFHNPNPEIPALLDGRLQVVDRPLPVRGGNVGINSFGFGGSNVHVILQPNDVSSSKPHPSAHKPRLFTYS ARTEHGLRAILREAQTHAASMEFHALCQASADAPLGSMPYRGATILNGQHDFEVVEKCKSKARE VWFIYAGMGSQWVGMARCLMQLDVFRHSLEKSAAVLKPHGVDLLNILSEGTEDTLRTILNPFVCI TSIQVALTDLLWSMGIRPDGIVGHSMGEVGCAYCDGCLTAEEAVLTAYWRGKCVTDGKVPPGKM AAVGLTWEEAKSQCPAGVVPACHNAEDSVTISGAADVMLKFMEELKAKDVFVREVNSSNIAYHS YFMENIYASLKDSLSKVISPKPRTARWLPTSVPEELWDSAPAQSSSAEFHANNLVSPVLFHEALQK IPPTAIAIELAPHGLLQSVIKRTLGNESVCVGLQKRNYADNLEFLFASLGKCFANGLSLNPLACYPPV EFPVPKGTPRLSDMVAGAWDHSAQWLVPKNEDFEGRVQASGSDSSYSIDVSAEKVKAAVEGEET VGQQVIKAVGNVLGLKSVSGVDPDKVFLDLGLDSLMSVEIKQMLERDLDLALGTKDIQMLTFAQL QAMVGGGSGGGSGNEVTTLENDAAFIENENAYLEKEIARLRKEKAALRNRLAHKKLEHHHHHH 


\section{Mathematical Examination of Changes in Transacylation Kinetic Parameters Caused by Point Mutations}

Kinetic parameters without bar correspond to the wild type (WT) DEBS3M5 KS-AT. Kinetic parameters with a bar correspond to the mutant (Mut).

$$
\begin{array}{ll}
\mathrm{k}_{\mathrm{cat}}:=\mathrm{k}_{\mathrm{cat}}(\mathrm{WT}), & \overline{\mathrm{k}}_{\mathrm{cat}}:=\mathrm{k}_{\mathrm{cat}}(\mathrm{Mut}), \\
\mathrm{K}_{\mathrm{m}}^{\mathrm{MMal}-\mathrm{CoA}}:=\mathrm{K}_{\mathrm{m}}^{\mathrm{MMal}-\mathrm{CoA}}(\mathrm{WT}), & \overline{\mathrm{K}}_{\mathrm{m}}^{\mathrm{MMal}-\mathrm{CoA}}:=\mathrm{K}_{\mathrm{m}}^{\mathrm{MMal}-\mathrm{CoA}} \text { (Mut), } \\
\mathrm{K}_{\mathrm{m}}^{\mathrm{ACP}}:=\mathrm{K}_{\mathrm{m}}^{\mathrm{ACP}}(\mathrm{WT}), & \overline{\mathrm{K}}_{\mathrm{m}}^{\mathrm{ACP}}:=\mathrm{K}_{\mathrm{m}}^{\mathrm{ACP}}(\mathrm{Mut}) .
\end{array}
$$

Kinetic parameters are described as follows:

$$
\begin{array}{ll}
\mathrm{k}_{\mathrm{cat}}=\frac{\mathrm{k}_{2} \mathrm{k}_{4}}{\mathrm{k}_{2}+\mathrm{k}_{4}}, & \overline{\mathrm{k}}_{\mathrm{cat}}=\frac{\overline{\mathrm{k}}_{2} \overline{\mathrm{k}}_{4}}{\mathrm{k}_{2}+\mathrm{k}_{4}}, \\
\mathrm{~K}_{\mathrm{m}}^{\mathrm{MMal}-\mathrm{CoA}}=\frac{\mathrm{k}_{4}\left(\mathrm{k}_{-1}+\mathrm{k}_{2}\right)}{\mathrm{k}_{1}\left(\mathrm{k}_{2}+\mathrm{k}_{4}\right)}, & \overline{\mathrm{K}}_{\mathrm{m}}^{\mathrm{MMal}-\mathrm{CoA}}=\frac{\overline{\mathrm{k}}_{4}\left(\overline{\mathrm{k}}_{-1}+\overline{\mathrm{k}}_{2}\right)}{\mathrm{k}_{1}\left(\mathrm{k}_{2}+\overline{\mathrm{k}}_{4}\right)}, \\
\mathrm{K}_{\mathrm{m}}^{\mathrm{ACP}}=\frac{\mathrm{k}_{2}\left(\mathrm{k}_{-3}+\mathrm{k}_{4}\right)}{\mathrm{k}_{3}\left(\mathrm{k}_{2}+\mathrm{k}_{4}\right)}, & \overline{\mathrm{K}}_{\mathrm{m}}^{\mathrm{ACP}}=\frac{\overline{\mathrm{k}}_{2}\left(\overline{\mathrm{k}}_{-3}+\overline{\mathrm{k}}_{4}\right)}{\overline{\mathrm{k}}_{3}\left(\overline{\mathrm{k}}_{2}+\overline{\mathrm{k}}_{4}\right)} .
\end{array}
$$

Kinetic analysis of the mutants revealed the following relationships of kinetic parameters:

$$
\begin{aligned}
\overline{\mathrm{k}}_{\mathrm{cat}} & =\alpha \mathrm{k}_{\mathrm{cat}}, \\
\overline{\mathrm{K}}_{\mathrm{m}}^{\mathrm{MMal}-\mathrm{CoA}} & =\beta \mathrm{K}_{\mathrm{m}}^{\mathrm{MMal}-\mathrm{CoA}}, \\
\overline{\mathrm{K}}_{\mathrm{m}}^{\mathrm{ACP}} & =\gamma \mathrm{K}_{\mathrm{m}}^{\mathrm{ACP}} .
\end{aligned}
$$

The following ratios of factors were found for mutants significantly changing kinetic parameters:

$$
\begin{array}{ll}
\text { R850K : } & \alpha=\beta \neq \gamma=1, \\
\text { R850E : } & \alpha=\gamma \neq \beta, \\
\text { R850S : } & \alpha \neq \beta=\gamma .
\end{array}
$$


All three mutants showed significant changes in the turnover rate. This can be caused by changes in either $\mathrm{k}_{2}$, or in $\mathrm{k}_{4}$, or in both constants.

$$
\text { (5.1) } \quad \Rightarrow \overline{\mathrm{k}}_{2} \overline{\mathrm{k}}_{4}=\left(\overline{\mathrm{k}}_{2}+\overline{\mathrm{k}}_{4}\right) \propto \frac{\mathrm{k}_{2} \mathrm{k}_{4}}{\mathrm{k}_{2}+\mathrm{k}_{4}} \text {. }
$$

This equation can be solved for $\overline{\mathrm{k}}_{4}$ or $\overline{\mathrm{k}}_{2}$, respectively:

$$
\begin{aligned}
\Rightarrow \overline{\mathrm{k}}_{4} & =\frac{\alpha \mathrm{k}_{2} \mathrm{k}_{4} \overline{\mathrm{k}}_{2}}{\overline{\mathrm{k}}_{2}\left(\mathrm{k}_{2}+\mathrm{k}_{4}\right)-\alpha \mathrm{k}_{2} \mathrm{k}_{4}}, \\
\overline{\mathrm{k}}_{2} & =\frac{\alpha \mathrm{k}_{2} \mathrm{k}_{4} \overline{\mathrm{k}}_{4}}{\overline{\mathrm{k}}_{4}\left(\mathrm{k}_{2}+\mathrm{k}_{4}\right)-\alpha \mathrm{k}_{2} \mathrm{k}_{4}} .
\end{aligned}
$$

The mutations introduced can affect either the ping, or the pong, or both steps:

Scenario 1: Ping step is not affected $\left(\overline{\mathrm{k}}_{1}=\mathrm{k}_{1}, \overline{\mathrm{k}}_{-1}=\mathrm{k}_{-1}, \overline{\mathrm{k}}_{2}=\mathrm{k}_{2}\right)$.

$$
\begin{gathered}
\text { (5.9) } \Rightarrow \overline{\mathrm{k}}_{4}=\frac{\alpha \mathrm{k}_{2} \mathrm{k}_{4}}{\mathrm{k}_{2}+(1-\alpha) \mathrm{k}_{4}} \\
\Rightarrow \mathrm{k}_{2}+\overline{\mathrm{k}}_{4}=\frac{\left[\mathrm{k}_{2}+(1-\alpha) \mathrm{k}_{4}\right] \mathrm{k}_{2}+\alpha \mathrm{k}_{2} \mathrm{k}_{4}}{\mathrm{k}_{2}+(1-\alpha) \mathrm{k}_{4}}=\frac{\mathrm{k}_{2}\left(\mathrm{k}_{2}+\mathrm{k}_{4}\right)}{\mathrm{k}_{2}+(1-\alpha) \mathrm{k}_{4}} . \\
\overline{\mathrm{k}}_{4} \text { into (5.2) } \quad \frac{\overline{\mathrm{k}}_{4}\left(\mathrm{k}_{1}+\mathrm{k}_{2}\right)}{\mathrm{k}_{1}\left(\mathrm{k}_{2}+\overline{\mathrm{k}}_{4}\right)}=\beta \frac{\mathrm{k}_{4}\left(\mathrm{k}_{1}+\mathrm{k}_{2}\right)}{\mathrm{k}_{1}\left(\mathrm{k}_{2}+\mathrm{k}_{4}\right)} \\
\Rightarrow \frac{\alpha \mathrm{k}_{2} \mathrm{k}_{4}}{\mathrm{k}_{2}\left(\mathrm{k}_{2}+\mathrm{k}_{4}\right)}=\beta \frac{\mathrm{k}_{4}}{\mathrm{k}_{2}+\mathrm{k}_{4}} \Rightarrow \alpha=\beta
\end{gathered}
$$

For mutants R850E and R850S, this finding leads to a contradiction and, hence, it can be ruled out that these two mutations affect solely the pong step, leaving the ping step unaffected. For mutation R850K on the other hand, this scenario is consistent. 
Scenario 2: Pong step is not affected $\left(\overline{\mathrm{k}}_{3}=\mathrm{k}_{3}, \overline{\mathrm{k}}_{-3}=\mathrm{k}_{-3}, \overline{\mathrm{k}}_{4}=\mathrm{k}_{4}\right)$.

$$
\begin{gathered}
\text { (5.10) } \quad \Rightarrow \overline{\mathrm{k}}_{2}=\frac{\alpha \mathrm{k}_{2} \mathrm{k}_{4}}{(1-\alpha) \mathrm{k}_{2}+\mathrm{k}_{4}} \\
\Rightarrow \overline{\mathrm{k}}_{2}+\mathrm{k}_{4}=\frac{\left[(1-\alpha) \mathrm{k}_{2}+\mathrm{k}_{4}\right] \mathrm{k}_{4}+\alpha \mathrm{k}_{2} \mathrm{k}_{4}}{(1-\alpha) \mathrm{k}_{2}+\mathrm{k}_{4}}=\frac{\mathrm{k}_{4}\left(\mathrm{k}_{2}+\mathrm{k}_{4}\right)}{(1-\alpha) \mathrm{k}_{2}+\mathrm{k}_{4}} . \\
\overline{\mathrm{k}}_{2} \text { into (5.3) } \quad \frac{\overline{\mathrm{k}}_{2}\left(\mathrm{k}_{3}+\mathrm{k}_{4}\right)}{\mathrm{k}_{3}\left(\overline{\mathrm{k}}_{2}+\mathrm{k}_{4}\right)}=\gamma \frac{\mathrm{k}_{2}\left(\mathrm{k}_{3}+\mathrm{k}_{4}\right)}{\mathrm{k}_{3}\left(\mathrm{k}_{2}+\mathrm{k}_{4}\right)} \\
\Rightarrow \frac{\alpha \mathrm{k}_{2} \mathrm{k}_{4}}{\mathrm{k}_{4}\left(\mathrm{k}_{2}+\mathrm{k}_{4}\right)}=\gamma \frac{\mathrm{k}_{2}}{\mathrm{k}_{2}+\mathrm{k}_{4}} \Rightarrow \alpha=\gamma .
\end{gathered}
$$

For mutants R850K and R850S, this finding leads to a contradiction and, hence, it can be ruled out that these two mutations affect solely the ping step, leaving the pong step unaffected. For mutation R850E on the other hand, this scenario is consistent.

In summary, the changes in kinetic parameters caused by mutant R850S cannot be explained by sole changes in either the ping or the pong step. This means that both steps are influenced by this mutation. 


\section{Appendix}

Scenario 3: Both steps are affected, exemplarily shown for mutant R850K.

$$
\begin{aligned}
& \overline{\mathrm{k}}_{4}=\frac{\alpha \mathrm{k}_{2} \mathrm{k}_{4} \overline{\mathrm{k}}_{2}}{\overline{\mathrm{k}}_{2}\left(\mathrm{k}_{2}+\mathrm{k}_{4}\right)-\alpha \mathrm{k}_{2} \mathrm{k}_{4}} \\
& \Rightarrow \overline{\mathrm{k}}_{2}+\overline{\mathrm{k}}_{4}=\frac{\left[\overline{\mathrm{k}}_{2}\left(\mathrm{k}_{2}+\mathrm{k}_{4}\right)-\alpha \mathrm{k}_{2} \mathrm{k}_{4}\right] \overline{\mathrm{k}}_{2}+\alpha \mathrm{k}_{2} \mathrm{k}_{4} \overline{\mathrm{k}}_{2}}{\overline{\mathrm{k}}_{2}\left(\mathrm{k}_{2}+\mathrm{k}_{4}\right)-\alpha \mathrm{k}_{2} \mathrm{k}_{4}} \\
&=\frac{\overline{\mathrm{k}}_{2}\left(\mathrm{k}_{2}+\mathrm{k}_{4}\right)}{\overline{\mathrm{k}}_{2}\left(\mathrm{k}_{2}+\mathrm{k}_{4}\right)-\alpha \mathrm{k}_{2} \mathrm{k}_{4}} \\
& \Rightarrow \frac{\overline{\mathrm{k}}_{4}}{\overline{\mathrm{k}}_{2}+\overline{\mathrm{k}}_{4}}=\frac{\alpha \mathrm{k}_{2} \mathrm{k}_{4}}{\overline{\mathrm{k}}_{2}\left(\mathrm{k}_{2}+\mathrm{k}_{4}\right)}
\end{aligned}
$$

$$
\Rightarrow \frac{1}{\overline{\mathrm{k}}_{1}}\left(\frac{\overline{\mathrm{k}}_{-1}}{\overline{\mathrm{k}}_{2}}+1\right)=\frac{1}{\mathrm{k}_{1}}\left(\frac{\mathrm{k}_{-1}}{\mathrm{k}_{2}}+1\right) .
$$

Changes of kinetic constants $\mathrm{k}_{\mathrm{i}}, \mathrm{i} \in\{-1,1,2\}$ do not result in a contradiction. However, the complex and dependent change of constants does seem unplausible, especially considering that the changes can be explained by effects on solely the pong step. This can be done analogously for mutant R850E.

Overall, it is most plausible to assume that R850K and R850E solely take effect on the pong step and the ping step, respectively.

Now that we know the relationships between $\overline{\mathrm{k}}_{2}$ and $\mathrm{k}_{2}$ as well as between $\overline{\mathrm{k}}_{4}$ and $\mathrm{k}_{4}$ for the mutants R850K and R850E, it remains to investigate whether the other constants involved in the affected step $\left(\mathrm{k}_{3}\right.$ and $\mathrm{k}_{3}$ in addition to $\mathrm{k}_{4}$ for $\mathrm{R} 850 \mathrm{~K}, \mathrm{k}_{1}$ and $\mathrm{k}_{-1}$ in addition to $\mathrm{k}_{2}$ for R850E) are influenced by the respective mutation. By a "proof of contradiction", I will show that $\overline{\mathrm{k}}_{3} \neq \mathrm{k}_{3}$ and $\overline{\mathrm{k}}_{-3} \neq \mathrm{k}_{-3}$ for R850K as well as $\overline{\mathrm{k}}_{1} \neq \mathrm{k}_{1}$ and $\overline{\mathrm{k}}_{-1} \neq \mathrm{k}_{-1}$ for R850E. 
R850K: Assume that solely $\mathrm{k}_{4}$ changes and $\overline{\mathrm{k}}_{3}=\mathrm{k}_{3}$ as well as $\overline{\mathrm{k}}_{-3}=\mathrm{k}_{-3}$.

$$
\begin{aligned}
\Rightarrow & \frac{\mathrm{k}_{2}\left(\mathrm{k}_{3}+\overline{\mathrm{k}}_{4}\right)}{\mathrm{k}_{3}\left(\mathrm{k}_{2}+\overline{\mathrm{k}}_{4}\right)}=\gamma \frac{\mathrm{k}_{2}\left(\mathrm{k}_{3}+\mathrm{k}_{4}\right)}{\mathrm{k}_{3}\left(\mathrm{k}_{2}+\mathrm{k}_{4}\right)} \\
& \frac{\mathrm{k}_{2}\left(\mathrm{k}_{3}+\frac{\alpha \mathrm{k}_{2} \mathrm{k}_{4}}{\mathrm{k}_{2}+(1-\alpha) \mathrm{k}_{4}}\right)\left[\mathrm{k}_{2}+(1-\alpha) \mathrm{k}_{4}\right]}{\mathrm{k}_{2} \mathrm{k}_{3}\left(\mathrm{k}_{2}+\mathrm{k}_{4}\right)}=\gamma \frac{\mathrm{k}_{2}\left(\mathrm{k}_{3}+\mathrm{k}_{4}\right)}{\mathrm{k}_{3}\left(\mathrm{k}_{2}+\mathrm{k}_{4}\right)} \\
\Rightarrow & \mathrm{k}_{-3}\left[\mathrm{k}_{2}+(1-\alpha) \mathrm{k}_{4}\right]+\alpha \mathrm{k}_{2} \mathrm{k}_{4}=\gamma \mathrm{k}_{2}\left(\mathrm{k}_{-3}+\mathrm{k}_{4}\right) \\
\Rightarrow & (1-\gamma) \mathrm{k}_{2} \mathrm{k}_{3}+(1-\alpha) \mathrm{k}_{3} \mathrm{k}_{4}=(\gamma-\alpha) \mathrm{k}_{2} \mathrm{k}_{4} .
\end{aligned}
$$

$$
\begin{aligned}
\text { with } \gamma=1 & \Rightarrow(1-\alpha) \mathrm{k}_{3} \mathrm{k}_{4}=(1-\alpha) \mathrm{k}_{2} \mathrm{k}_{4} \\
& \Rightarrow \mathrm{k}_{2}=\mathrm{k}_{-3} .
\end{aligned}
$$

Although this is not a contradiction in a strict mathematical sense, it represents a very specific situation. From a chemical point of view, there is no reason why this relation should hold as $\mathrm{k}_{2}$ and $\mathrm{k}_{3}$ are independent constants. This leads me to the aforementioned conclusion that for the mutation R850K most likely $\overline{\mathrm{k}}_{3} \neq \mathrm{k}_{3}$ and $\overline{\mathrm{k}}_{-3} \neq \mathrm{k}_{-3}$ in addition to $\overline{\mathrm{k}}_{4} \neq \mathrm{k}_{4}$.

R850E: Assuming that $\overline{\mathrm{k}}_{1}=\mathrm{k}_{1}$ and $\overline{\mathrm{k}}_{-1}=\mathrm{k}_{-1}$ using the above relation (5.2) gives the corresponding equation to (5.26):

$$
(1-\beta) \mathrm{k}_{4} \mathrm{k}_{-1}+(1-\alpha) \mathrm{k}_{-1} \mathrm{k}_{2}=(\beta-\alpha) \mathrm{k}_{2} \mathrm{k}_{4}
$$

Since $\beta \neq 1$, this cannot be further simplified. However, the kinetic constant $\mathrm{k}_{-1}$ is dependent on $\mathrm{k}_{2}$ and $\mathrm{k}_{4}$ :

$$
\Rightarrow \mathrm{k}_{-1}=\frac{(\beta-\alpha) \mathrm{k}_{2} \mathrm{k}_{4}}{(1-\alpha) \mathrm{k}_{2}+(1-\beta) \mathrm{k}_{4}}
$$

As discussed above, from a mathematical point of view it cannot be ruled out that the kinetic constants $\mathrm{k}_{1}$ and $\mathrm{k}_{-1}$ remain unchanged. However, it is unplausible that the kinetic constants are dependent and, hence, $\mathrm{I}$ assume these two change $\left(\overline{\mathrm{k}}_{1} \neq \mathrm{k}_{1}\right.$ and $\left.\overline{\mathrm{k}}_{1} \neq \mathrm{k}_{-1}\right)$ in addition to $\mathrm{k}_{2}\left(\overline{\mathrm{k}}_{2} \neq \mathrm{k}_{2}\right)$. 


\section{Supplementary Tables}

Table S1: Average expression yields (in $\mathrm{mg}$ ) of FAS and PKS AT domains after tandem affinity chromatography of $1 \mathrm{~L}$ expression culture. Yields determined from three independent expressions.

\begin{tabular}{lc}
\hline Protein & Yield (mg) \\
\hline DEBS3 $\left(\mathrm{KS}^{0}\right.$-AT) 5 & 14 \\
PikAIII $\left(\mathrm{KS}^{0}\right.$-AT)5 & 14 \\
RAPS3 (KS ${ }^{0}$-AT)14 & 3.5 \\
Pks7 KS ${ }^{0}$-AT & 22 \\
DEBS1 AT0-BMP & 7.6 \\
AVES1 AT0 & 12 \\
FabD & 12 \\
\hline
\end{tabular}

Table S2: TSA results of FAS and PKS AT and ACP domains. Average melting temperatures (in ${ }^{\circ} \mathrm{C}$ ) of biological triplicates (ATs) and technical triplicates (ACPs). n. d.: not determinable.

\begin{tabular}{lcccc}
\hline Protein & \multicolumn{2}{c}{ AT } & \multicolumn{2}{c}{ ACP } \\
\hline & Storage Buffer & Assay Buffer & Storage Buffer & Assay Buffer \\
\hline DEBS3M5 & 51 & 42 & 64 & 65 \\
PikAllIM5 & 49 & 42 & 59 & 56 \\
RAPS3M14 & 45 & 36 & 57 & 50 \\
Pks7 & 49 & 41 & 62 & 58 \\
DEBS1M0 & 48 & 42 & 63 & 59 \\
AVES1M0 & 55 & 49 & 53 & 55 \\
E. coli & 65 & 61 & n.d. & n.d. \\
\hline
\end{tabular}


Table S3: Average expression yields (in $\mathrm{mg}$ ) of non-codon optimized and codon optimized (COP) holo-ACP domains after SEC of $1 \mathrm{~L}$ expression culture. Yields determined from four to nine independent expressions.

\begin{tabular}{lc}
\hline Protein & Yield (mg) \\
\hline DEBS3 ACP5 & 23 \\
DEBS3 ACP5 COP & 87 \\
PikAIII ACP5 & 4.5 \\
PikAIII ACP5 COP & 51 \\
RAPS3 ACP14 & 31 \\
Pks7 ACP & 1.0 \\
Pks7 ACP COP & 30 \\
DEBS1 ACP0 & 9.4 \\
AVES1 ACP0 & 11 \\
E. coli ACP COP & 29 \\
\hline
\end{tabular}




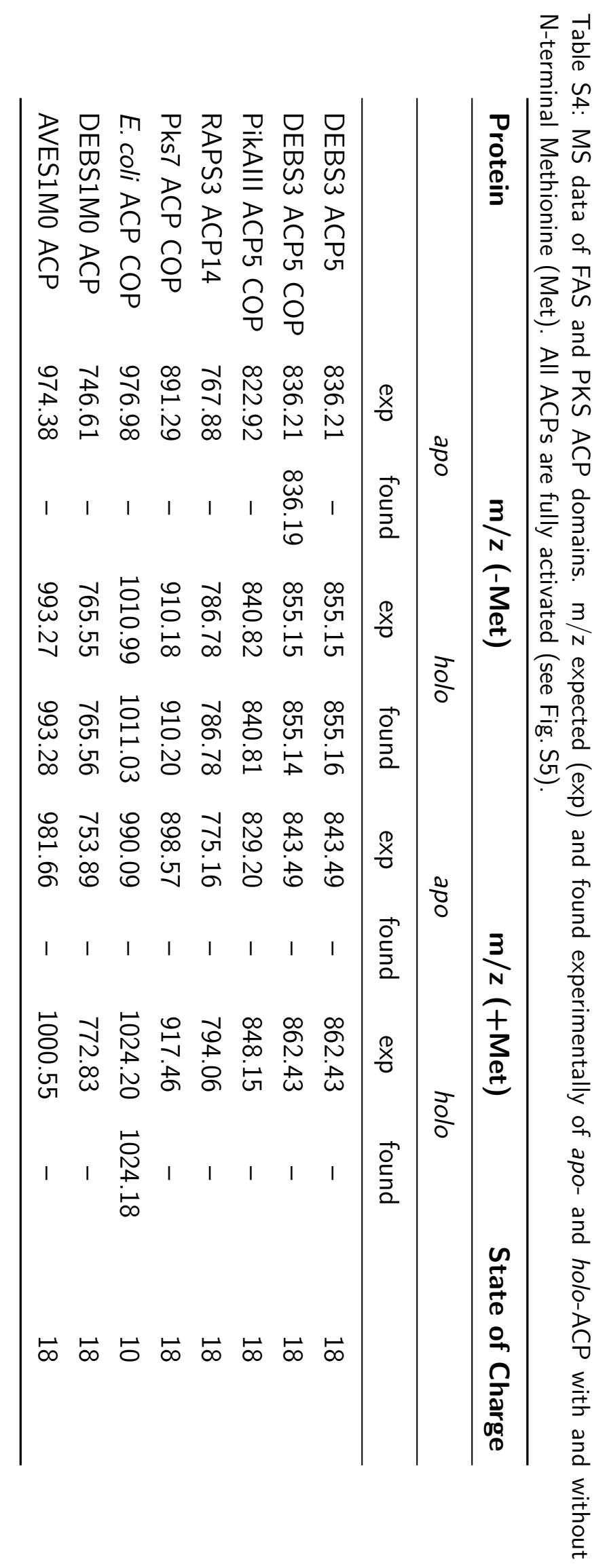


Table S5: Transacylation and hydrolysis rates (in s $\left.{ }^{-1}\right)$ of initial substrate screening $(50 \mu \mathrm{M}$ XCoA and ACP) of FAS and PKS AT domains. Transacylation measured in biological triplicates. Hydrolysis measured in technical triplicates of each biological triplicate. [a] mMAT from previous study, referring to $k_{c a t}$ for hydrolysis (saturated $\mathrm{X}-\mathrm{CoA}$ ) and $\mathrm{k}_{\mathrm{cat}}$, app for transacylation (saturated X-CoA, $60 \mu \mathrm{M}$ ACP). [9]

\begin{tabular}{|c|c|c|c|}
\hline Protein & X-CoA & $\begin{array}{c}\text { Transacylation } \\
\mathrm{k}_{0, \text { app }}\left(\mathrm{s}^{-1}\right)\end{array}$ & $\begin{array}{c}\text { Hydrolysis } \\
k_{0}\left(s^{-1}\right)\end{array}$ \\
\hline DEBS3 AT5 & MMal & $1.92 \times 10^{-1}$ & $4.24 . \times 10^{-2}$ \\
\hline PikAIII AT5 & Mal & $8.86 \times 10^{-2}$ & $4.98 \times 10^{-2}$ \\
\hline PikAIII AT5 & MMal & $8.60 \times 10^{-1}$ & $1.54 \times 10^{-2}$ \\
\hline RAPS3 AT14 & Mal & $2.05 \times 10^{-1}$ & $6.63 \times 10^{-2}$ \\
\hline Pks7 AT & $A c$ & $2.81 \times 10^{-1}$ & $1.60 \times 10^{-3}$ \\
\hline Pks7 AT & Mal & 2.79 & $7.05 \times 10^{-2}$ \\
\hline Pks7 AT & MMal & 1.69 & $5.58 \times 10^{-2}$ \\
\hline DEBS1 AT0 & Prop & $7.59 \times 10^{-2}$ & $3.11 \times 10^{-2}$ \\
\hline DEBS1 AT0 & $A c$ & $3.67 \times 10^{-2}$ & $3.00 \times 10^{-2}$ \\
\hline AVES1 AT0 & $2-\mathrm{MB}$ & $5.49 \times 10^{-1}$ & $4.89 \times 10^{-2}$ \\
\hline AVES1 AT0 & Prop & $-4.03 \times 10^{-3}$ & $2.72 \times 10^{-2}$ \\
\hline $\mathrm{mMAT}^{[\mathrm{a}]}$ & Ac & 13.7 & $9.3 \times 10^{-3}$ \\
\hline $\operatorname{mMAT}[\mathrm{a}]$ & Mal & 15.6 & $9.8 \times 10^{-3}$ \\
\hline mMAT[a] & MMal & 4.0 & $6.0 \times 10^{-3}$ \\
\hline FabD & $A c$ & 3.19 & $6.77 \times 10^{-3}$ \\
\hline FabD & Mal & 93.9 & $5.00 \times 10^{-3}$ \\
\hline FabD & MMal & 72.4 & $3.71 \times 10^{-4}$ \\
\hline
\end{tabular}


Table S6: Kinetic parameters determined for AT-mediated hydrolysis. Turnover measured in technical triplicates of biological triplicates (DEBS3 AT5), technical triplicates of one replicate (PikAIII AT5, RAPS3 AT14), or biological triplicates (Pks7 with Mal and MMal). [a] Value experimentally determined in hydrolysis measurement. [b] Value calculated from experimentally determined hydrolysis and transacylation values. [c] Value experimentally determined in transacylation measurement. [d] mMAT from previous study. [9]

\begin{tabular}{|c|c|c|c|}
\hline $\begin{array}{l}\text { Protein } \\
\text { X-CoA }\end{array}$ & $k_{\text {cat }}\left(s^{-1}\right)^{[a]}$ & $\mathrm{K}_{\mathrm{m}}^{\mathrm{X}-\operatorname{CoA}}(\mu \mathrm{M})^{[\mathrm{b}]}$ & $\begin{array}{c}\mathrm{k}_{\mathrm{cat}} / \mathrm{K}_{\mathrm{m}}^{\mathrm{X}-\mathrm{CoA}} \\
\left(\mathrm{s}^{-1} \mathrm{M}^{-1}\right)^{[c]}\end{array}$ \\
\hline $\begin{array}{l}\text { DEBS3 AT5 } \\
\text { MMal }\end{array}$ & $5.31 \times 10^{-2} \pm 9.62 \times 10^{-4}$ & 8.85 & $6.0 \times 10^{3}$ \\
\hline $\begin{array}{l}\text { PikAlll AT5 } \\
\text { MMal }\end{array}$ & $1.94 \times 10^{-2} \pm 5.03 \times 10^{-4}$ & $2.49 \times 10^{-1}$ & $7.8 \times 10^{4}$ \\
\hline $\begin{array}{l}\text { RAPS3 AT14 } \\
\text { Mal }\end{array}$ & $6.51 \times 10^{-2} \pm 1.89 \times 10^{-3}$ & 1.36 & $4.8 \times 10^{4}$ \\
\hline $\begin{array}{l}\text { Pks7 AT } \\
\text { Mal }\end{array}$ & $9.93 \times 10^{-2} \pm 3.17 \times 10^{-3}$ & $3.97 \times 10^{-2}$ & $2.5 \times 10^{6}$ \\
\hline $\begin{array}{l}\text { Pks7 AT } \\
\text { MMal }\end{array}$ & $7.34 \times 10^{-2} \pm 1.51 \times 10^{-3}$ & $7.57 \times 10^{-2}$ & $9.7 \times 10^{5}$ \\
\hline $\begin{array}{l}\text { mMAT } \\
\text { Mal[d] }\end{array}$ & $(9.8 \pm 1.7) \times 10^{-3}$ & $7.0 \times 10^{-4}$ & $1.4 \times 10^{7}$ \\
\hline $\begin{array}{l}\text { mMAT } \\
A_{c}[\mathrm{~d}]\end{array}$ & $9.3 \times 10^{-3} \pm 1.2 \times 10^{-4}$ & $1.1 \times 10^{-3}$ & $8.3 \times 10^{6}$ \\
\hline
\end{tabular}


Table S7: Average expression yields (in mg) of DEBS3 (KS ${ }^{0}$-AT) 5 interface mutants after tandem affinity chromatography of $1 \mathrm{~L}$ expression culture. Data from three independent protein expressions, except proteins marked with ${ }^{*}$ are single expressions.

\begin{tabular}{cc}
\hline Mutation & Yield (mg) \\
\hline A539S $^{*}$ & 10 \\
A539D $^{*}$ & 15 \\
A539E & 9.7 \\
A539F & 1.7 \\
R850K & 14 \\
R850A & 5.7 \\
R850E & 7.7 \\
R850F & 2.4 \\
R850S & 9.6 \\
\hline
\end{tabular}

Table S8: TSA results of DEBS3 $\left(\mathrm{KS}^{0}-\mathrm{AT}\right) 5$ interface mutants. Average melting temperatures (in ${ }^{\circ} \mathrm{C}$ ) of technical/biological triplicates. DEBS3M5 ACP COP was used in the mutagenesis study.

\begin{tabular}{cccc}
\hline Mutation & AT & \multicolumn{2}{c}{ ACP } \\
\hline & Storage Buffer & Storage Buffer & Assay Buffer \\
\hline- & 51 & 63 & 62 \\
A539S & 48 & - & - \\
A539D & 48 & - & - \\
A539E & 48 & - & - \\
A539F & 48 & - & - \\
R850K & 48 & - & - \\
R850A & 48 & - & - \\
R850E & 47 & - & - \\
R850F & 48 & - & - \\
R850S & 48 & - & - \\
\hline
\end{tabular}




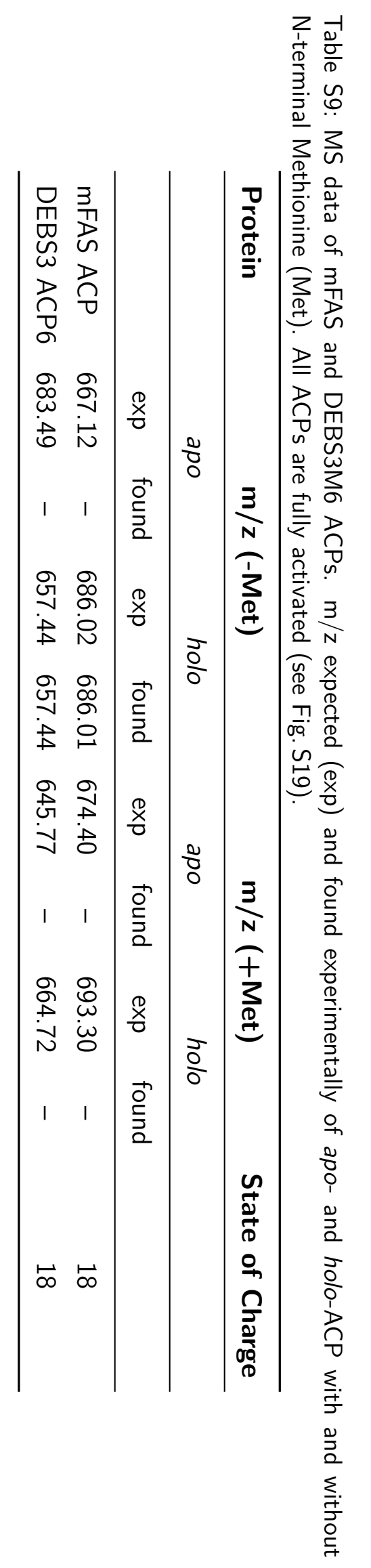




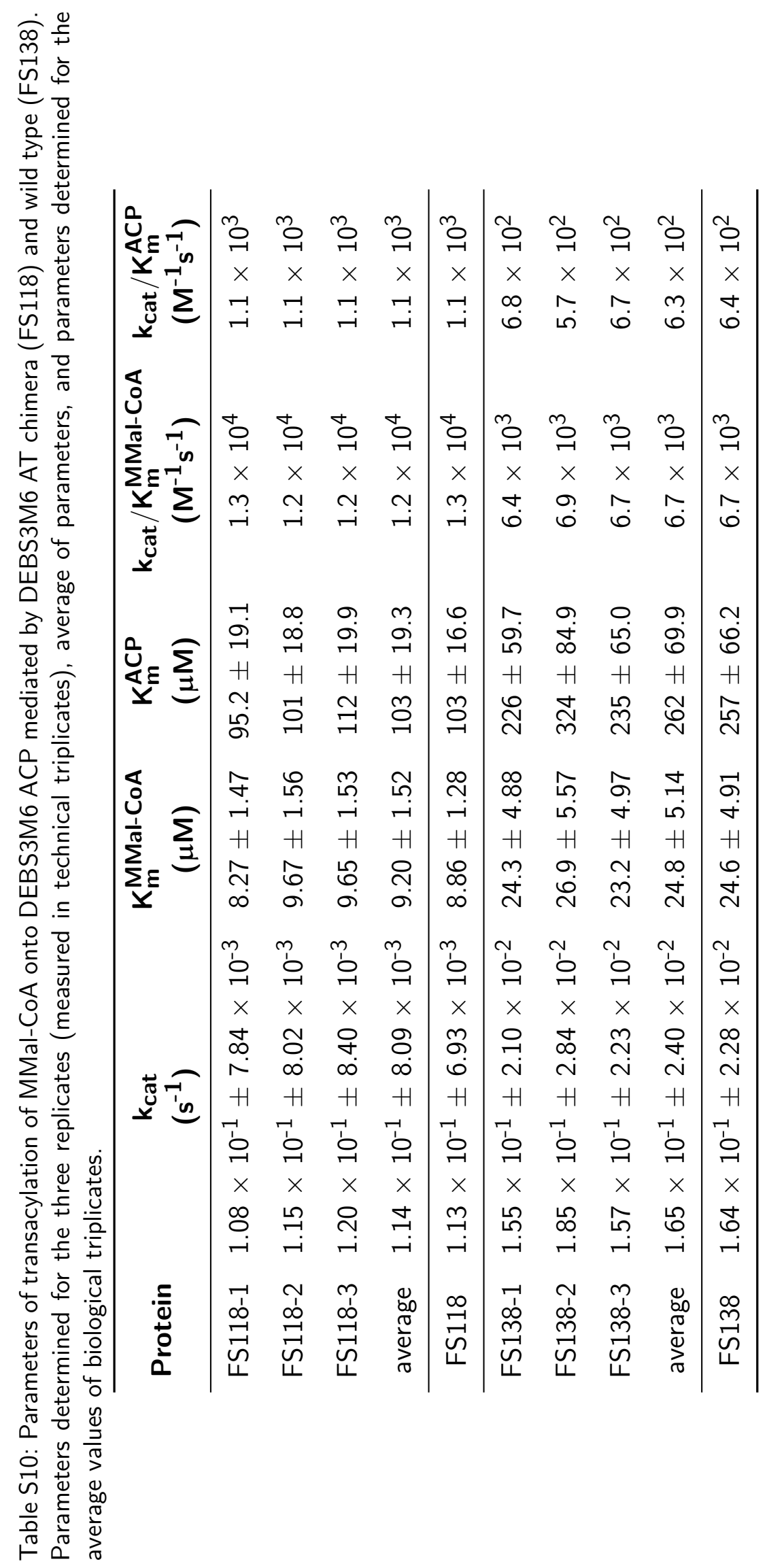


Table S11: NADH calibration factor over time determined via internal standard measurements for AT activity assay in 96 -well format.

\begin{tabular}{ll}
\hline Time Span & Calibration Factor $(\mathrm{RFU} / \mu \mathrm{M})$ \\
\hline $22 / 02 / 2018-27 / 03 / 2018$ & 13450 \\
$07 / 11 / 2018-05 / 12 / 2018$ & 11950 \\
$07 / 08 / 2019-27 / 09 / 2019$ & 11064 \\
$31 / 10 / 2019-18 / 11 / 2019$ & 9058 \\
$03 / 03 / 2020-06 / 03 / 2020$ & 8596 \\
\hline
\end{tabular}




\section{Supplementary Figures}
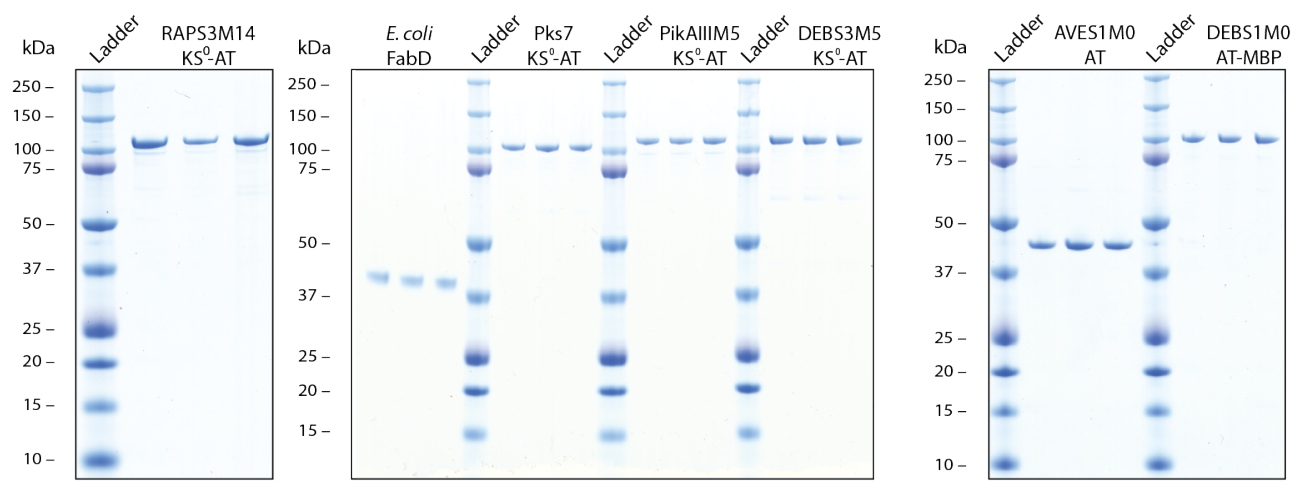

Figure S1: Analytical SDS-PAGEs (NuPAGE Bis-Tris 4-12\%) of FAS and PKS AT constructs expressed in biological triplicates. All AT constructs are highly pure after tandem affinity chromatography. Protein bands migrate at expected masses: RAPS3M14 KS ${ }^{0}$-AT - $101 \mathrm{kDa}$, E. coli FabD - 36.8 kDa, Pks7 KS ${ }^{0}$ AT - 98.6 kDa, PikAllIM5 KS ${ }^{0}-\mathrm{AT}-101 \mathrm{kDa}$, DEBS3M5 $\mathrm{KS}^{0}$-AT $-99.9 \mathrm{kDa}$, AVES1M0 AT - 42.8 kDa, DEBS1M0 AT-MBP - 94.1 kDa. 


\section{Appendix}
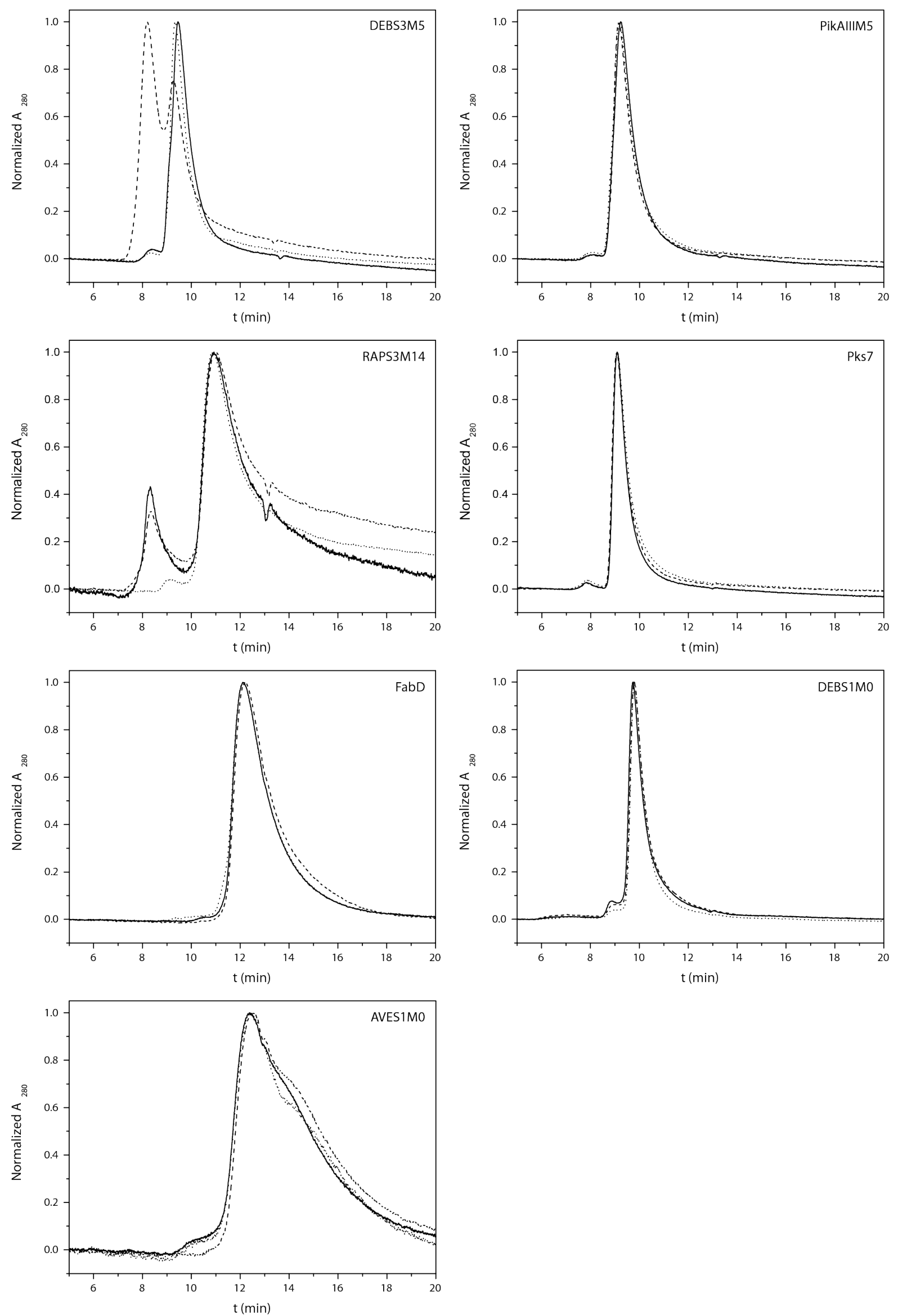
Figure S2: Normalized size exclusion chromatograms of FAS and PKS AT constructs. Each curve (solid, dotted, dashed) corresponds to one biological replicate. DEBS3M5 KS ${ }^{0}$-AT shows dimeric $(9.4 \mathrm{~min})$ and tetrameric $(8.2 \mathrm{~min})$ oligomers. PikAllIM5 $\mathrm{KS}^{0}$-AT forms solely dimers $(9.2 \mathrm{~min})$. RAPS3M14 $\mathrm{KS}^{0}$-AT shows monomeric $(11 \mathrm{~min})$ and tetrameric $(8.3 \mathrm{~min})$ species. We note that the prevalence for a monomeric state should not impact AT activity, since the dimeric interface is formed by the $\mathrm{KS}$ domain, while the AT domain is monomeric in its active form. Pks7 KS ${ }^{0}$-AT forms solely dimers $(9.1 \mathrm{~min})$. E. coli FabD, DEBS1M0 AT-MBP, and AVES1M0 ATs are solely monomeric $(12.1 \mathrm{~min}, 9.8 \mathrm{~min}$, and $12.5 \mathrm{~min})$. 

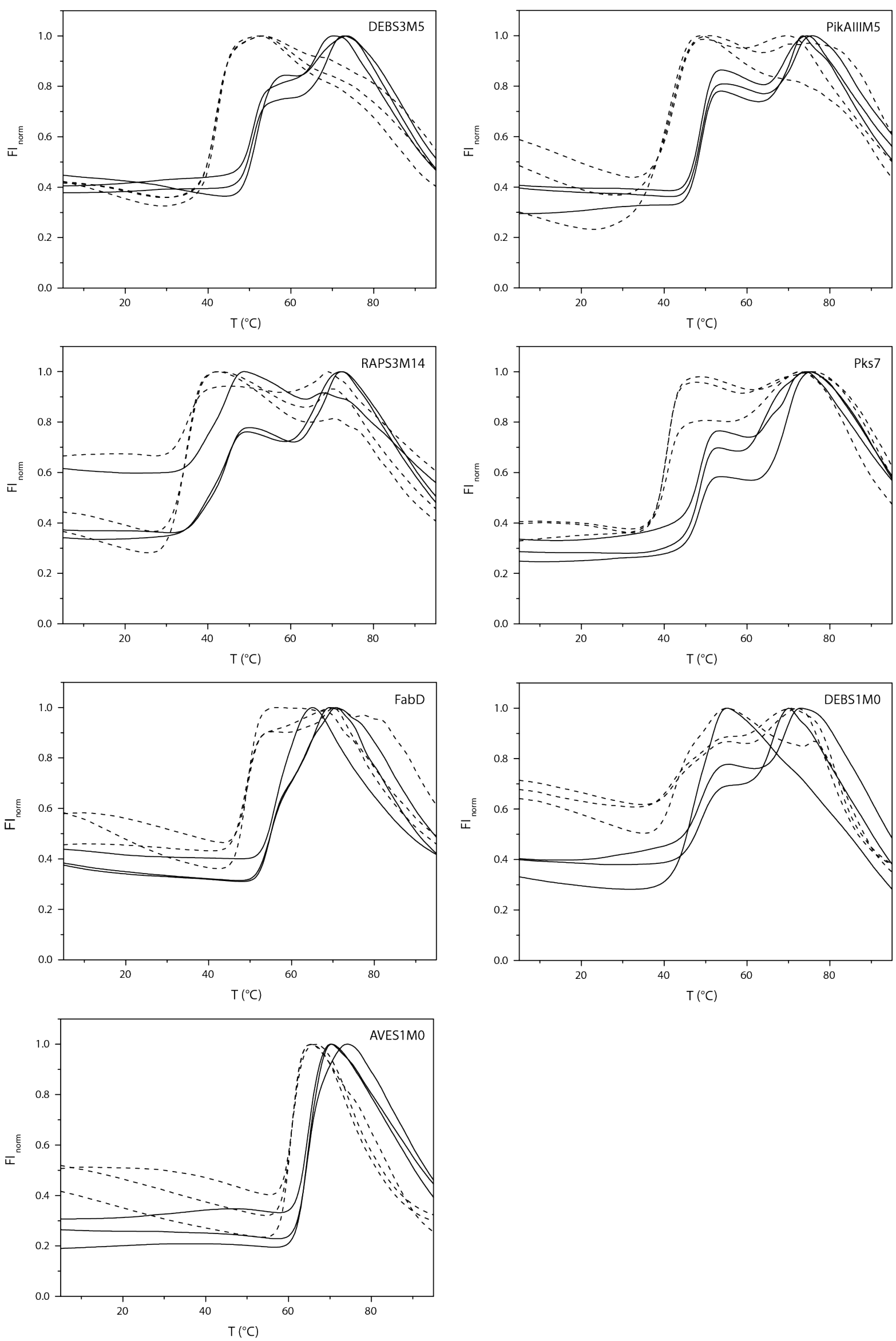
Figure S3: Normalized TSA melting curves of FAS and PKS (KS $\left.{ }_{-}^{-}\right)$AT domains. Solid and dashed line display data collected in storage and assay buffer, respectively. Each curve (solid, dashed) corresponds to one biological replicate.

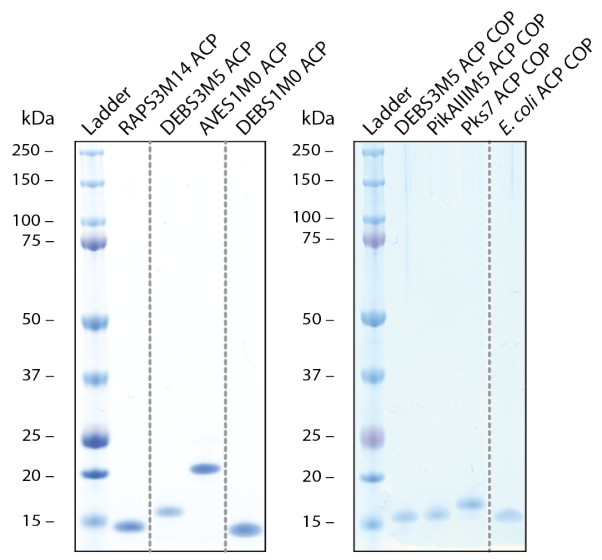

Figure S4: Analytical SDS-PAGEs (NuPAGE Bis-Tris 4-12\%) of FAS and PKS ACP domains. All pooled ACPs are highly pure after SEC. Masses are as expected: RAPS3M14 ACP $14.1 \mathrm{kDa}$, DEBS3M5 ACP (COP) - $15.4 \mathrm{kDa}$, PikAllIM5 ACP COP - $15.2 \mathrm{kDa}$, Pks7 ACP COP - 16.4kDa, AVES1M0 ACP - 17.7 kDa, DEBS1M0 ACP - 13.6kDa. E. coli ACP is known to run in gel higher than expected from its mass of $10.2 \mathrm{kDa}$. 


\section{Appendix}

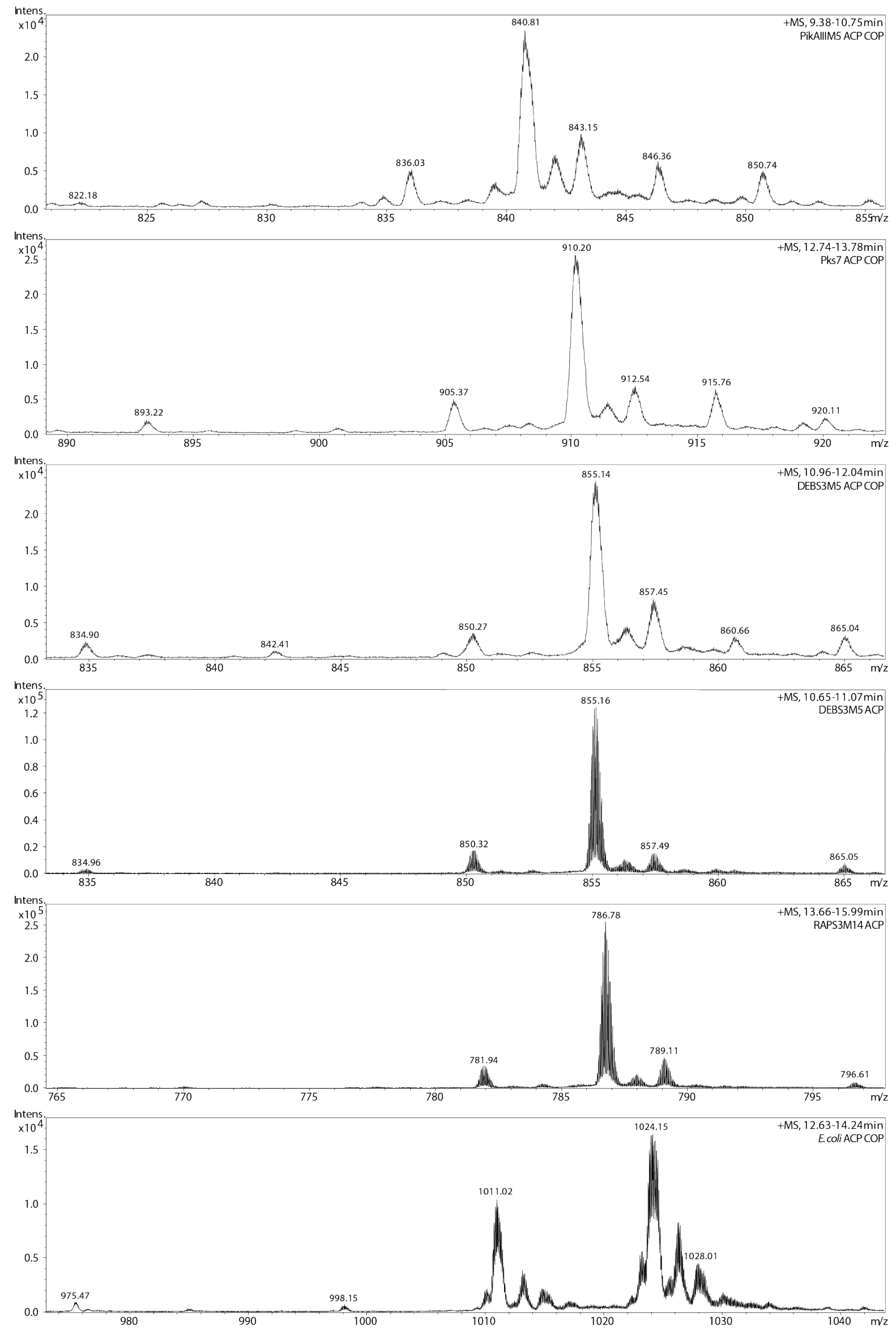




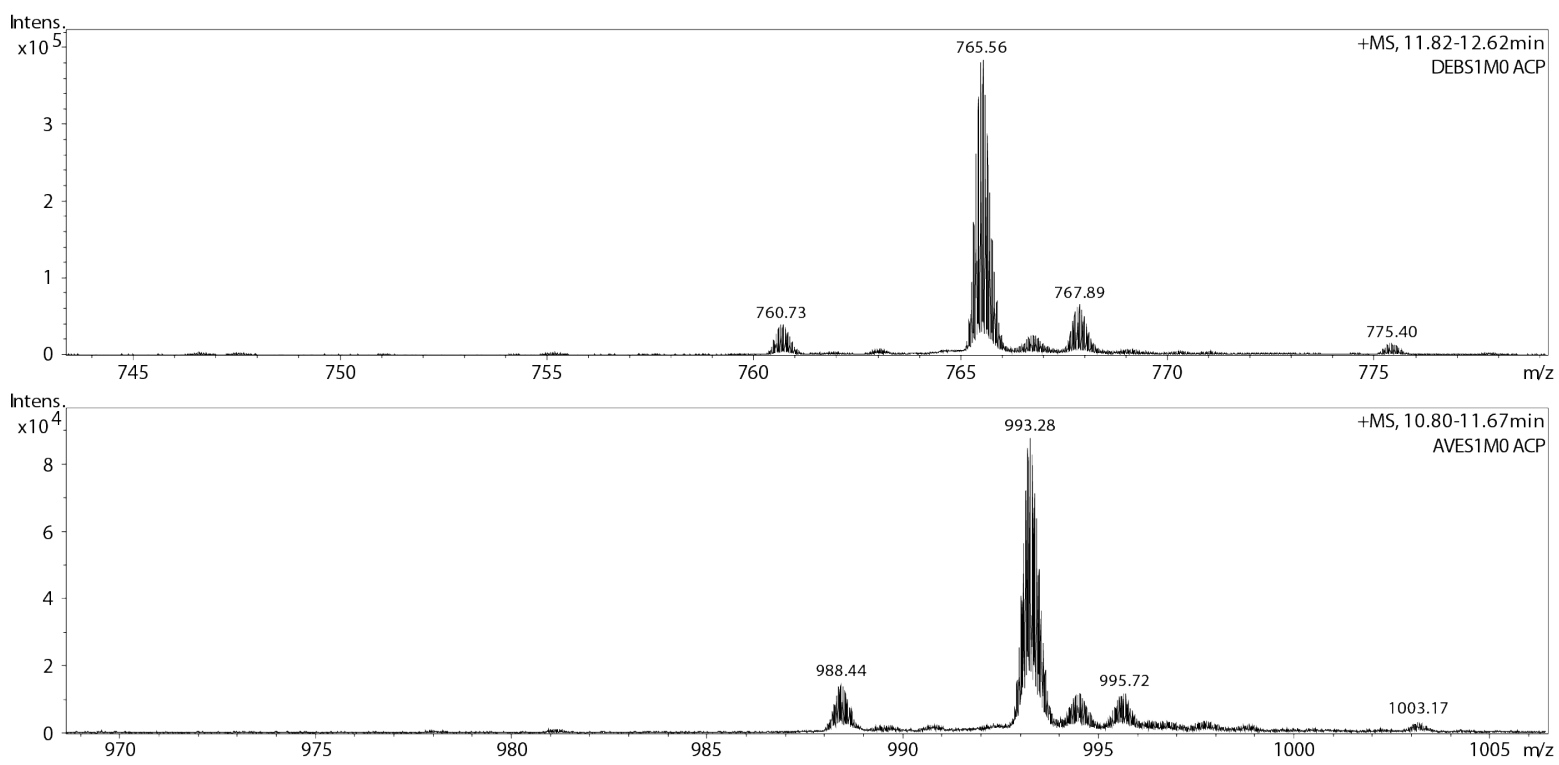

Figure S5: MS analysis of FAS and PKS ACP domains after SEC. Shown are the $\mathrm{m} / \mathrm{z}$-values of the average protein masses of the $10^{+}$and $18^{+}$charge states $\left(\mathrm{MS}^{1}\right)$ for $E$. coli and all other proteins, respectively. In none of the samples, $\mathrm{m} / \mathrm{z}$-values of the apo species were recorded. Main $\mathrm{m} / \mathrm{z}$-values correspond to the holo form. E. coli ACP shows two species: one with and one without $\mathrm{N}$-terminal methionine. All other proteins do not include the $\mathrm{N}$-terminal methionine. COP stands for codon optimized. PikAllIM5 ACP COP, $\mathrm{z}=18 \mathrm{~m} \mathrm{~m} / \mathrm{z}=$ 840.81 corresponds to holo-ACP without $\mathrm{N}$-terminal methionine. $\mathrm{m} / \mathrm{z}=843.15$ corresponds to acetylation $(\Delta \mathrm{m}=42 \mathrm{Da}), \mathrm{m} / \mathrm{z}=850.74$ corresponds to $\alpha$-N-gluconozylation $(\Delta \mathrm{m}=$ $178 \mathrm{Da}) . \mathrm{m} / \mathrm{z}=846.36$ is an unknown modified variant $(\Delta \mathrm{m}=100 \mathrm{Da}) . \mathrm{m} / \mathrm{z}=836.03$ corresponds to holo-ACP without $\mathrm{N}$-terminal methionine and serine $(\Delta \mathrm{m}=-86 \mathrm{Da})$. Pks7 ACP COP, $z=18: \mathrm{m} / \mathrm{z}=910.20$ corresponds to holo-ACP without $\mathrm{N}$-terminal methionine. $\mathrm{m} / \mathrm{z}=912.54$ corresponds to acetylation $(\Delta \mathrm{m}=42 \mathrm{Da}), \mathrm{m} / \mathrm{z}=920.11$ corresponds to $\alpha$ $\mathrm{N}$-gluconozylation $(\Delta \mathrm{m}=178 \mathrm{Da}) . \mathrm{m} / \mathrm{z}=915.76$ is an unknown modified variant $(\Delta \mathrm{m}=$ $100 \mathrm{Da}) . \mathrm{m} / \mathrm{z}=905.37$ corresponds to holo-ACP without $\mathrm{N}$ - terminal methionine and serine $(\Delta \mathrm{m}=-87 \mathrm{Da})$. DEBS3M5 ACP COP, $\mathrm{z}=18$, used for kinetic analysis of mutants: $\mathrm{m} / \mathrm{z}=$ 855.14 corresponds to holo-ACP without $\mathrm{N}$-terminal methionine. $\mathrm{m} / \mathrm{z}=857.45$ corresponds to acetylation $(\Delta \mathrm{m}=42 \mathrm{Da}), \mathrm{m} / \mathrm{z}=865.04$ corresponds to $\alpha$-N-gluconozylation $(\Delta \mathrm{m}=178 \mathrm{Da})$. $\mathrm{m} / \mathrm{z}=860.66$ is an unknown modified variant $(\Delta \mathrm{m}=99 \mathrm{Da}) . \mathrm{m} / \mathrm{z}=850.27$ corresponds to holo-ACP without $\mathrm{N}$-terminal methionine and serine $(\Delta \mathrm{m}=-88 \mathrm{Da})$. DEBS3M5 ACP, $\mathrm{z}=18$, used for kinetic analysis of the wild type: $\mathrm{m} / \mathrm{z}=855.16$ corresponds to holo-ACP without $\mathrm{N}$-terminal methionine. $\mathrm{m} / \mathrm{z}=857.49$ corresponds to acetylation $(\Delta \mathrm{m}=42 \mathrm{Da}), \mathrm{m} / \mathrm{z}$ $=865.05$ corresponds to $\alpha$-N-gluconozylation $(\Delta \mathrm{m}=178 \mathrm{Da}) . \mathrm{m} / \mathrm{z}=860.66$ is an unknown modified variant $(\Delta \mathrm{m}=99 \mathrm{Da}) . \mathrm{m} / \mathrm{z}=850.32$ corresponds to holo-ACP without $\mathrm{N}$-terminal methionine and serine $(\Delta \mathrm{m}=-87 \mathrm{Da})$. RAPS3M14 ACP, $\mathrm{z}=18: \mathrm{m} / \mathrm{z}=786.78$ corresponds to holo-ACP without $\mathrm{N}$-terminal methionine. $\mathrm{m} / \mathrm{z}=789.11$ corresponds to acetylation $(\Delta \mathrm{m}$ $=42 \mathrm{Da}), \mathrm{m} / \mathrm{z}=796.61$ corresponds to $\alpha-\mathrm{N}$-gluconozylation $(\Delta \mathrm{m}=177 \mathrm{Da}) . \mathrm{m} / \mathrm{z}=781.94$ corresponds to holo-ACP without $\mathrm{N}$-terminal methionine and serine $(\Delta \mathrm{m}=-87 \mathrm{Da})$. E. coli ACP COP, $z=10: \mathrm{m} / \mathrm{z}=1011.02$ corresponds to holo-ACP including the N-terminal methionine. $\mathrm{m} / \mathrm{z}=1015.28$ corresponds to the acetylated form $(\Delta \mathrm{m}=43 \mathrm{Da}) . \mathrm{m} / \mathrm{z}=$ 1024.15 corresponds to holo-ACP without $\mathrm{N}$-terminal methionine. $\mathrm{m} / \mathrm{z}=1028.01$ corresponds to acetylation $(\Delta \mathrm{m}=39 \mathrm{Da}), \mathrm{m} / \mathrm{z}=1042.00$ corresponds to $\alpha$-N-gluconozylation $(\Delta \mathrm{m}=$ $178 \mathrm{Da}$ ). DEBS1M0 ACP, $\mathrm{z}=18 \mathrm{~m} / \mathrm{z}=765.56$ corresponds to holo-ACP without $\mathrm{N}$ terminal methionine. $\mathrm{m} / \mathrm{z}=767.89$ corresponds to acetylation $(\Delta \mathrm{m}=42 \mathrm{Da}), \mathrm{m} / \mathrm{z}=775.40$ 
corresponds to $\alpha$-N-gluconozylation $(\Delta \mathrm{m}=177 \mathrm{Da}) . \mathrm{m} / \mathrm{z}=760.73$ corresponds to holo-ACP without $\mathrm{N}$-terminal methionine and serine $(\Delta \mathrm{m}=-87 \mathrm{Da})$. AVES1M0 ACP, $\mathrm{z}=18: \mathrm{m} / \mathrm{z}=$ 993.28 corresponds to holo-ACP without $\mathrm{N}$-terminal methionine. $\mathrm{m} / \mathrm{z}=995.72$ corresponds to acetylation $(\Delta \mathrm{m}=44 \mathrm{Da}), \mathrm{m} / \mathrm{z}=1003.17$ corresponds to $\alpha$-N-gluconozylation $(\Delta \mathrm{m}=$ $178 \mathrm{Da}) . \mathrm{m} / \mathrm{z}=988.44$ corresponds to holo-ACP without $\mathrm{N}$-terminal methionine and serine $(\Delta \mathrm{m}=-87 \mathrm{Da})$. 

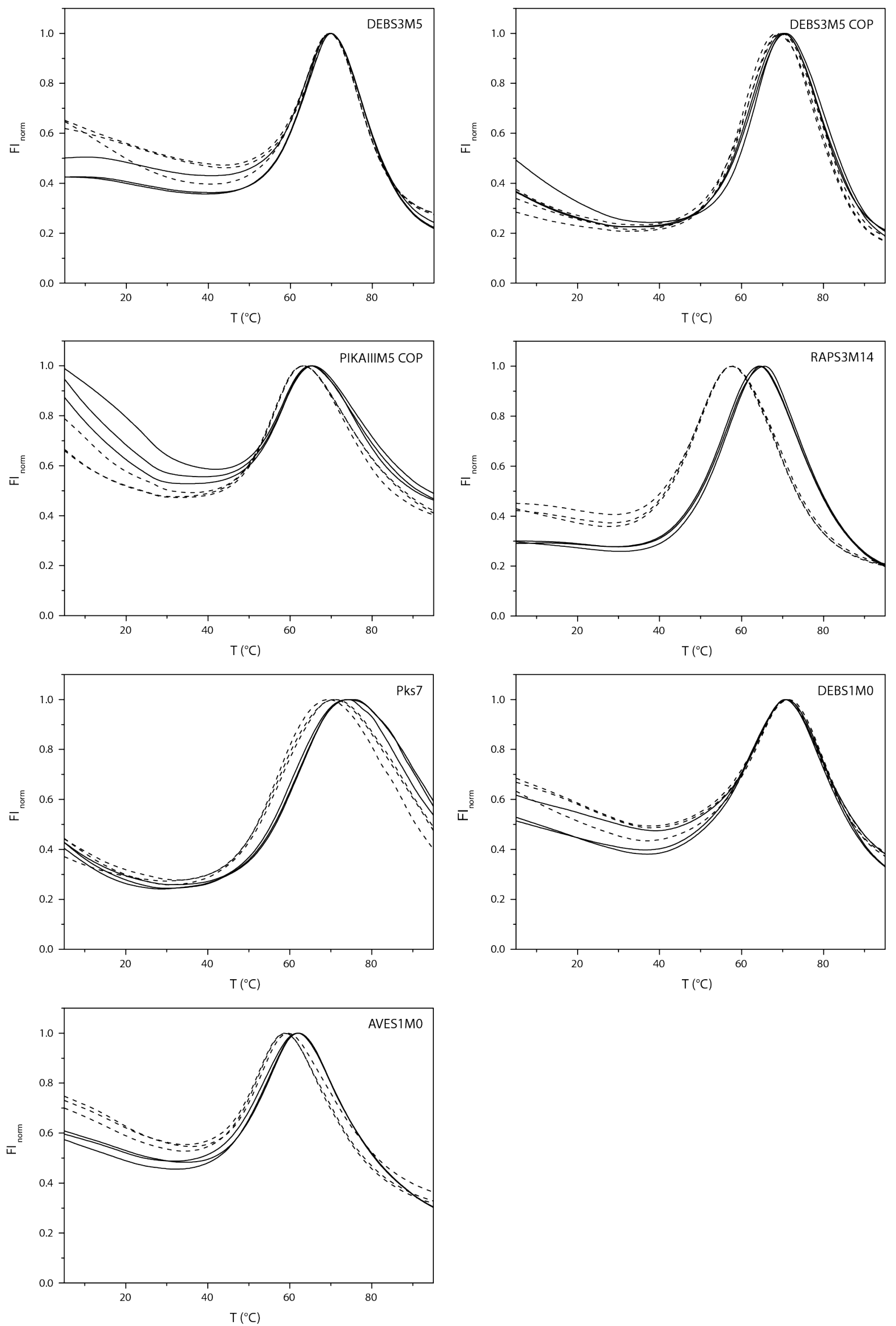
Figure S6: Normalized TSA melting curves of FAS and PKS ACP domains. Solid and dashed line display data collected in storage and assay buffer, respectively. Each curve (solid, dashed) corresponds to one technical replicate. COP stands for codon optimized. 

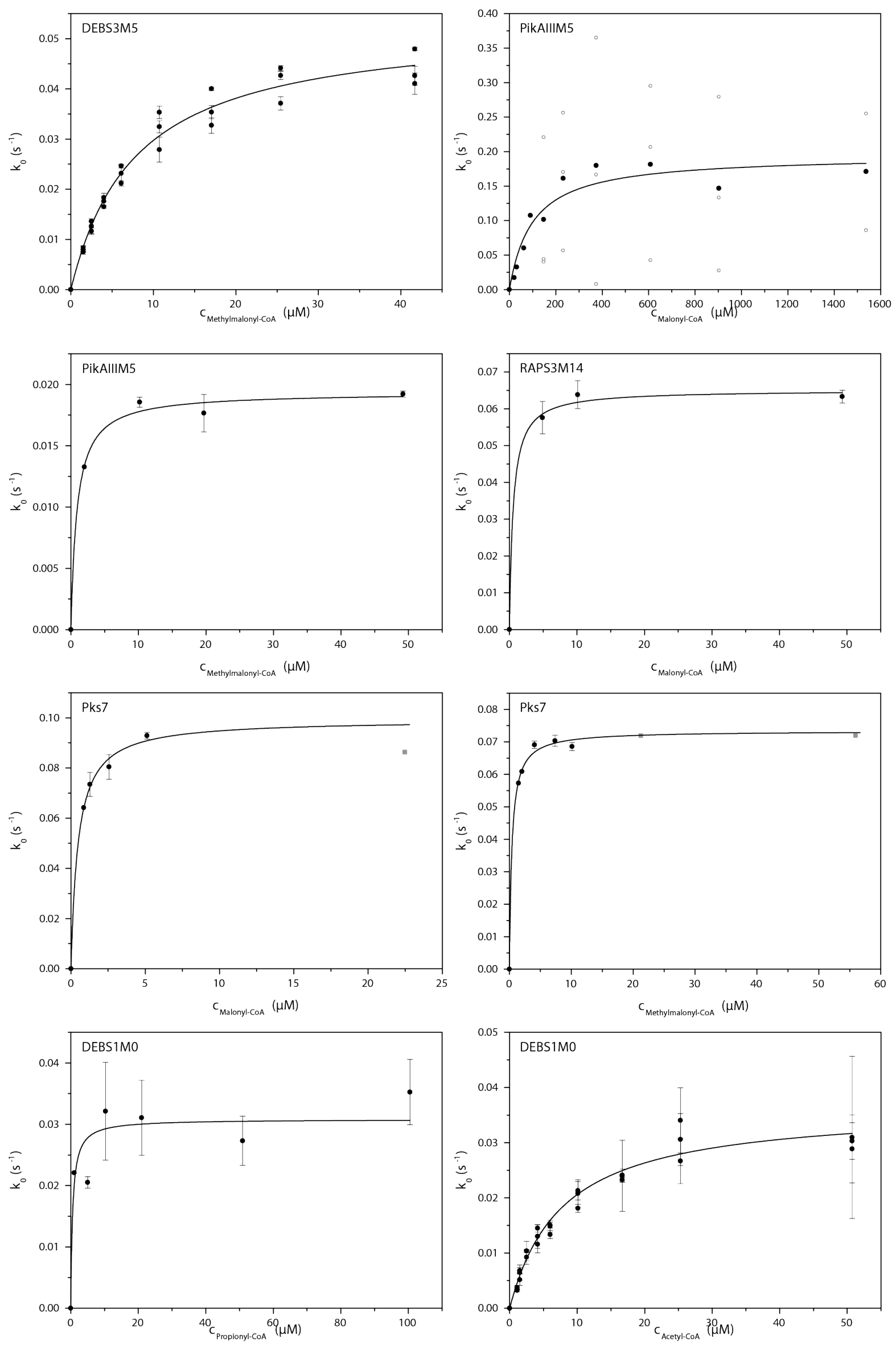


\section{Appendix}
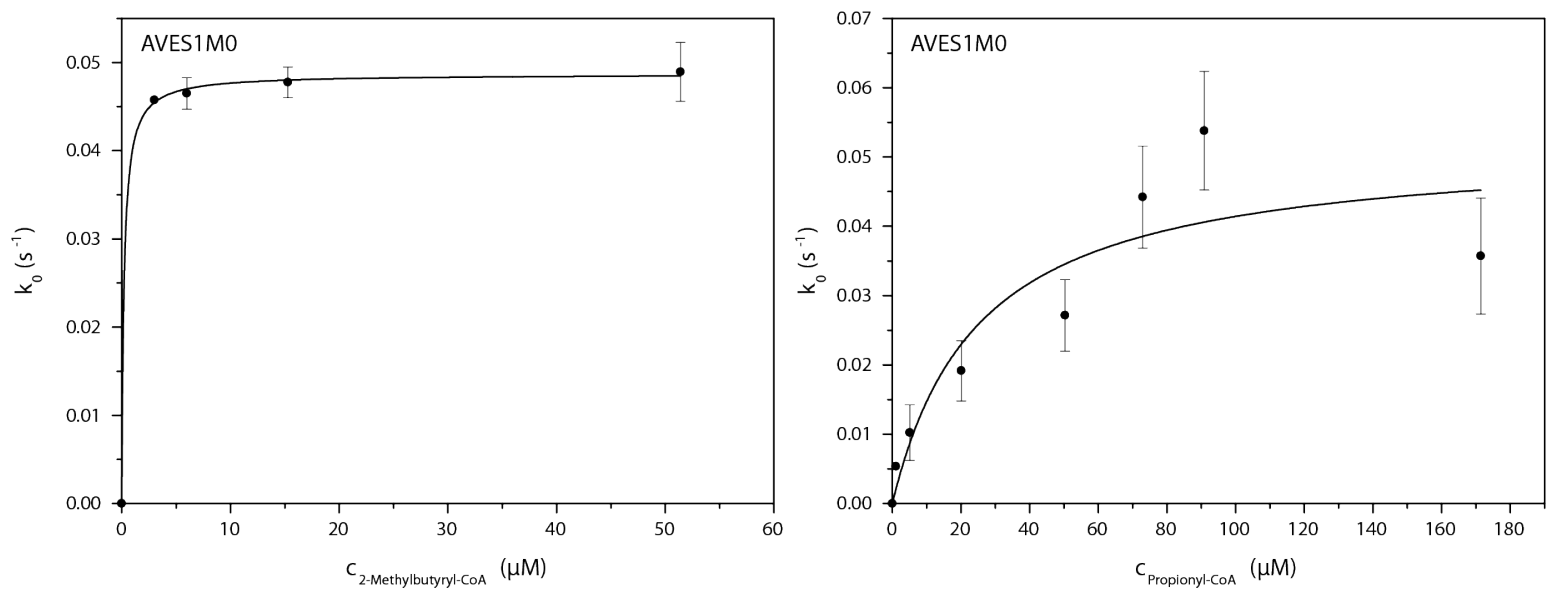

Figure S7: Titration curves for AT-mediated hydrolysis of X-CoA. DEBS3M5, MMal: DEBS3M5 $\mathrm{KS}^{0}$-AT measured in technical triplicates of biological triplicates. Black dots with error bars show the average of one biological replicate with standard deviation. Kinetic parameters were determined precisely. Substrate consumption was below $10 \%$. MMal range was 0.19-5.3 $\times \mathrm{K}_{\mathrm{m}}^{\mathrm{MMal}-\mathrm{CoA}}$. PikAlIIM5, Mal: PikAllIM5 $\mathrm{KS}^{0}-\mathrm{AT}$ measured in biological triplicates. Circles show one biological replicate, black dots show their average. Problems with high $\mathrm{Mal}$ concentrations were observed. Kinetic parameters with substantial errors were calculated. Substrate consumption was below $5 \%$. PikAllıM5, MMal: PikAllIM5 KS ${ }^{0}$-AT measured in technical triplicates. Black dots with error bars show the average with standard deviation. The lowest concentration is a one well measurement. Due to the low substrate concentrations, the maximum velocity and an upper limit of the Michaelis-Menten constant were determined for this system. Substrate consumption was up to $17 \%$. RAPS3M14, Mal: RAPS3M14 $\mathrm{KS}^{0}$-AT measured in technical triplicates. Black dots with error bars show the average with standard deviation. Due to the low substrate concentration, the maximum velocity and an upper limit of the Michaelis-Menten constant were determined. Substrate consumption was up to $24 \%$. Pks7, Mal: Pks7 KS ${ }^{0}$-AT measured in biological triplicates. Black dots with error bars show the average with standard deviation. The lowest concentration is a one well measurement. The gray box is a single well measurement with high substrate concentration, which was not used for the hydrolysis fit. It shows that maximum velocity was reached. Due to the low substrate concentrations, the maximum velocity and an upper limit of the Michaelis-Menten constant were determined. Substrate consumption was up to $44 \%$. Signal increase was still constant. Pks7, MMal: Pks7 KS ${ }^{0}$-AT measured in biological triplicates. Black dots with error bars show the average with standard deviation. The two lowest concentrations are one well measurements. Grey boxes are single well measurements with high substrate concentrations, which were not used for the hydrolysis fit. These values show that maximum velocity was reached. Due to the low substrate concentration, the maximum velocity and an upper limit of the Michaelis-Menten constant were determined. Substrate consumption was up to $34 \%$. Signal increase was still constant. DEBS1M0, Prop: DEBS1M0 AT-MBP measured in technical triplicates. Black dots with error bars show the average with standard deviation. The lowest concentration is a one well measurement. Due to the low substrate concentrations, the maximum velocity and an upper limit of the Michaelis-Menten constant were determined for this system. Substrate consumption was up to $20 \%$. DEBS1M0, Ac: DEBS1M0 AT-MBP measured in technical triplicates of biological triplicates. Black dots with error bars show the average of one biological replicate with standard deviation. Kinetic parameters were determined precisely. Substrate consumption was up to $11 \%$. Ac range was 0.14-5.4 $\times \mathrm{K}_{\mathrm{m}}^{\mathrm{Ac}-\mathrm{CoA}}$. AVES1M0, 2-MB: AVES1M0 AT measured in technical triplicates. Black dots with error bars show the average with 
standard deviation. The lowest concentration is a one well measurement. Due to the low substrate concentrations, the maximum velocity and an upper limit of the Michaelis-Menten constant were determined for this system. Substrate consumption was up to $14 \%$. AVES1M0, Prop: AVES1M0 AT measured in technical triplicates. Black dots with error bars show the average with standard deviation. The lowest concentration is a one well measurement. Due to large signal fluctuations kinetic parameters with substantial errors were calculated. Substrate consumption was up to $16 \%$. Prop range was $0.040-6.8 \times \mathrm{K}_{\mathrm{m}}^{\text {Prop-CoA }}$. 


\section{Appendix}
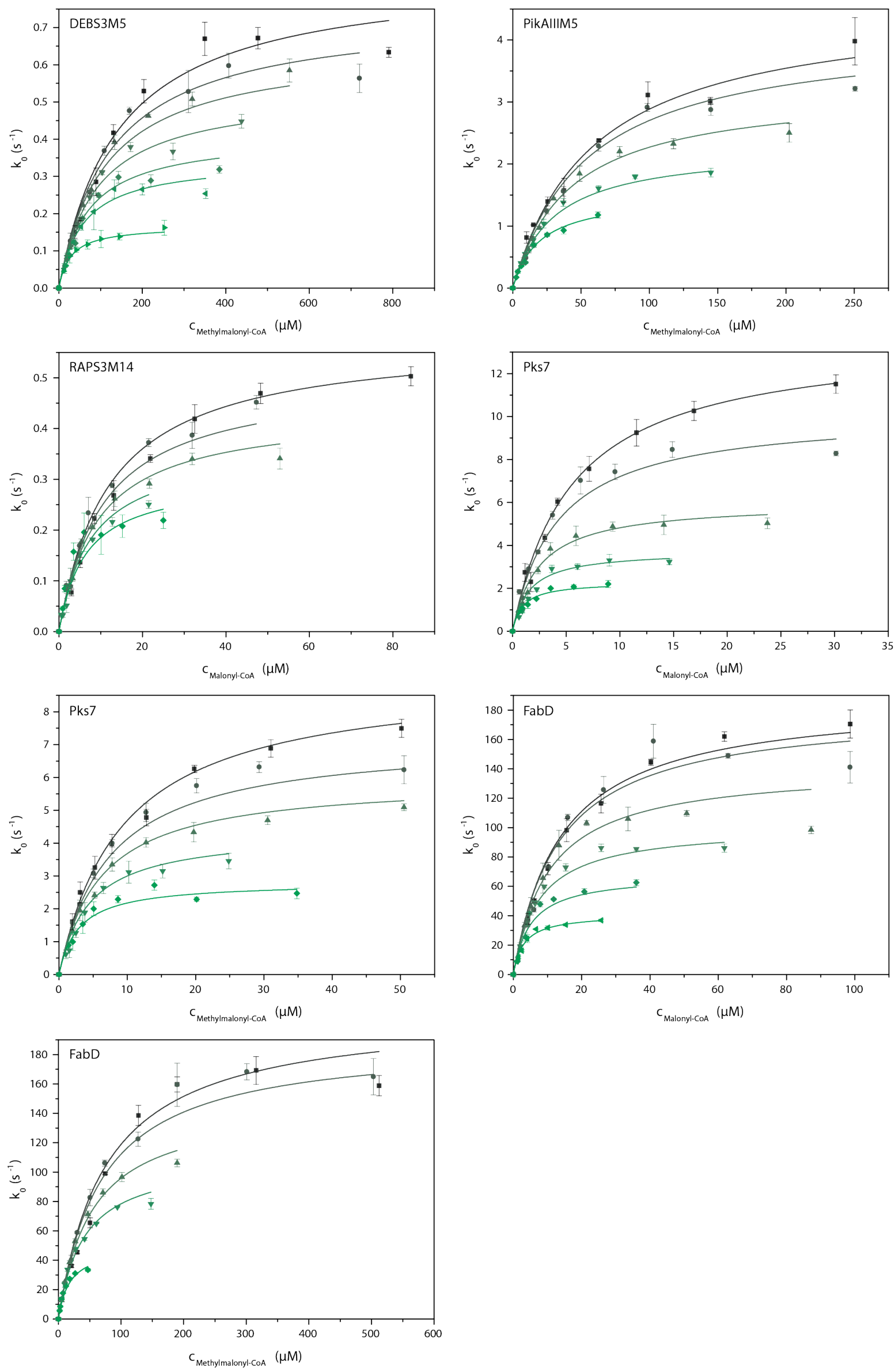
Figure S8: Global Michaelis-Menten fits of transacylation titration curves with X-CoA of AT domains. All measured in biological triplicates. Each color and symbol corresponds to one $X-C o A$ titration curve at a fixed ACP concentration. Error bars give the standard deviation of biological triplicates. ACP concentration as follows: DEBS3M5: $11.1 \mu \mathrm{M}, 27.2 \mu \mathrm{M}, 35.4 \mu \mathrm{M}, 53.1 \mu \mathrm{M}$, $78.2 \mu \mathrm{M}, 104.9 \mu \mathrm{M}$ and 152.1 $\mu \mathrm{M}$, PikAlIIM5: $83.3 \mu \mathrm{M}, 143.9 \mu \mathrm{M}, 249.2 \mu \mathrm{M}, 412.9 \mu \mathrm{M}$ and 528.4 $\mu \mathrm{M}$, RAPS3M14: $48.9 \mu \mathrm{M}, 70.1 \mu \mathrm{M}, 103.2 \mu \mathrm{M}, 156.7 \mu \mathrm{M}$ and $287.3 \mu \mathrm{M}$, Pks7, Mal: $31.2 \mu \mathrm{M}, 54.5 \mu \mathrm{M}, 98.6 \mu \mathrm{M}, 220.7 \mu \mathrm{M}$ and $403.6 \mu \mathrm{M}$, Pks7, MMal: $80.3 \mu \mathrm{M}, 136.0 \mu \mathrm{M}$, $206.3 \mu \mathrm{M}, 273.1 \mu \mathrm{M}$ and $402.5 \mu \mathrm{M}$, FabD, Mal: $8.0 \mu \mathrm{M}, 15.0 \mu \mathrm{M}, 26.7 \mu \mathrm{M}, 49.2 \mu \mathrm{M}$, $91.0 \mu \mathrm{M}, 103.8 \mu \mathrm{M}$, FabD, MMal: $10.0 \mu \mathrm{M}, 30.4 \mu \mathrm{M}, 52.9 \mu \mathrm{M}, 102.6 \mu \mathrm{M}, 148.6 \mu \mathrm{M}$. ACP concentration ranges as follows: DEBS3M5: $0.15-2.1 \times \mathrm{K}_{\mathrm{m}}^{\mathrm{ACP}}$, PikAllIM5: $0.25-1.6 \times$ $\mathrm{K}_{\mathrm{m}}^{\mathrm{ACP}}$, RAPS3M14: 0.74-4.4 $\times \mathrm{K}_{\mathrm{m}}^{\mathrm{ACP}}$, Pks7, Mal: 0.11-1.4 $\times \mathrm{K}_{\mathrm{m}}^{\mathrm{ACP}}, \mathrm{Pks}$, MMal: $0.16-$ $0.80 \times \mathrm{K}_{\mathrm{m}}^{\mathrm{ACP}}$, FabD, Mal: $\times \mathrm{K}_{\mathrm{m}}^{\mathrm{ACP}}$, FabD, MMal: $\times \mathrm{K}_{\mathrm{m}}^{\mathrm{ACP}}$. Substrate consumption as follows: DEBS3M5: below 5\%, PikAllIM5: below $11 \%$, RAPS3M14: up to $36 \%$, but signal increase was still linear, Pks7, Mal: up to $42 \%$, but signal increase was still linear, Pks7, MMal: up to $18 \%$, FabD, Mal: up to $28 \%$, but signal increase was still linear FabD, MMal: up to $19 \%$. X-CoA concentration ranges as follows: DEBS3M5: $0.16-8.3 \times$

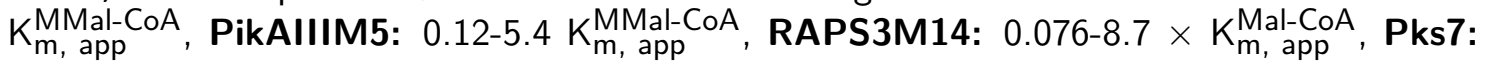
0.19-9.5 $\times \mathrm{K}_{\mathrm{m}, \mathrm{app}}^{\mathrm{Mal}-\mathrm{Co}}$ and 0.19-12 $\times \mathrm{K}_{\mathrm{m} \text {, app }}^{\mathrm{MMa}-\mathrm{CoA}}$, FabD: 0.23-13 $\times \mathrm{K}_{\mathrm{m}, \mathrm{app}}^{\mathrm{Mal}}$ and 0.12-7.2 $\times$ $\mathrm{K}_{\mathrm{m}, \mathrm{app}}^{\mathrm{MMal}-\mathrm{CoA}}$ 


\section{Appendix}

A
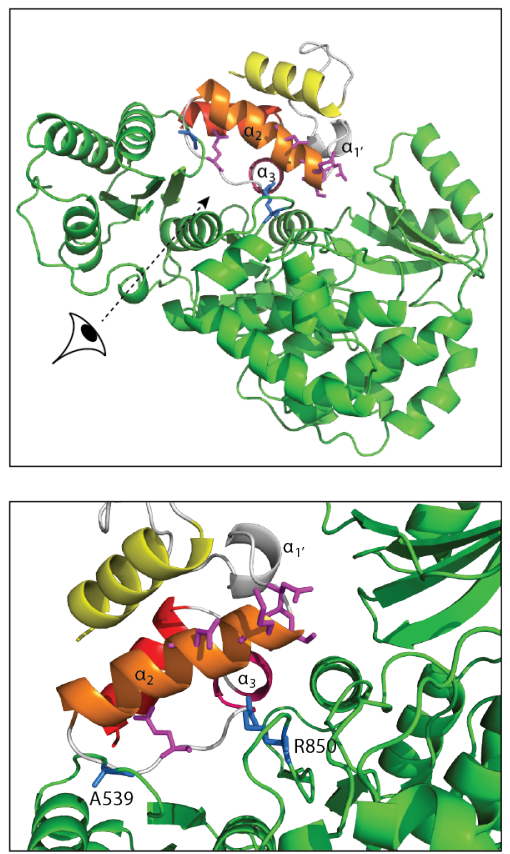

B
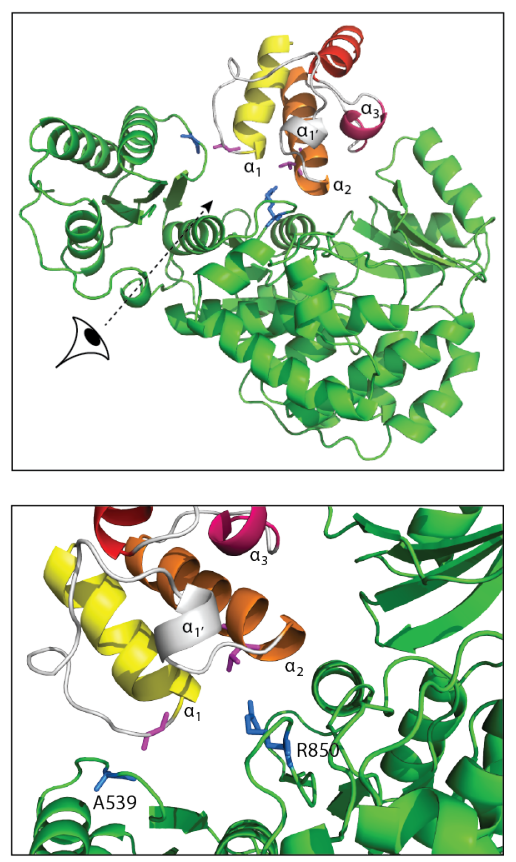

C
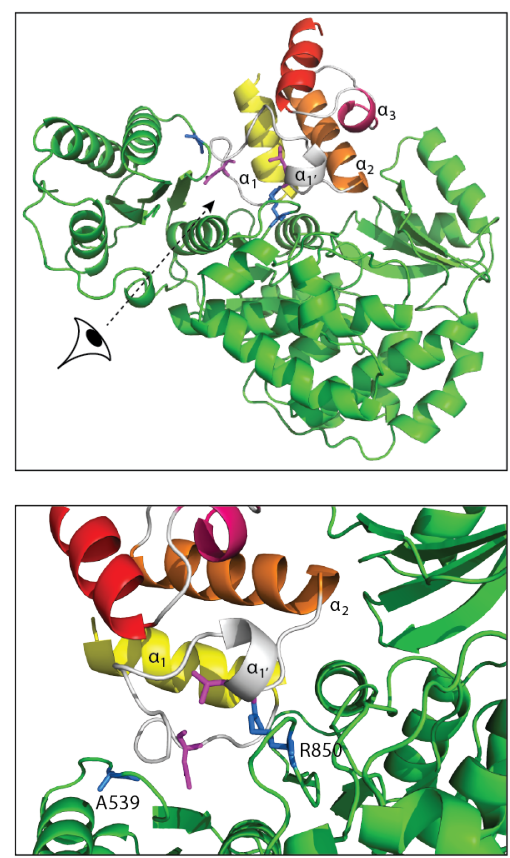

Figure S9: Detailed cartoon representation of the modeled DEBS3M5 AT:ACP interface. DEBS3M5 AT (PDB: 2HG4, shown in green) alignment with the standalone AT from disorazole synthase (PDB: 5ZK4) (A), with the standalone AT VinK from vicenistatin synthase (PDB: 5CZD) (B), and with MAT from human FAS (C). ${ }^{[125,126,159]}$ Upper panel shows an overview of the AT-ACP interaction of each alignment with perspective eyes for the detailed view showed in the lower panel. Residues A539 and R850 are highlighted in blue. Model DEBS3M5 ACP was aligned with the corresponding ACPs of templates, colored from yellow (N-terminus) to red (C-terminus). Interfacial amino acids suggested by PISA are highlighted in magenta. Despite their different AT:ACP interfaces, all models locate amino acids A539 and R850 (highlighted in blue) in the AT:ACP interfaces. 


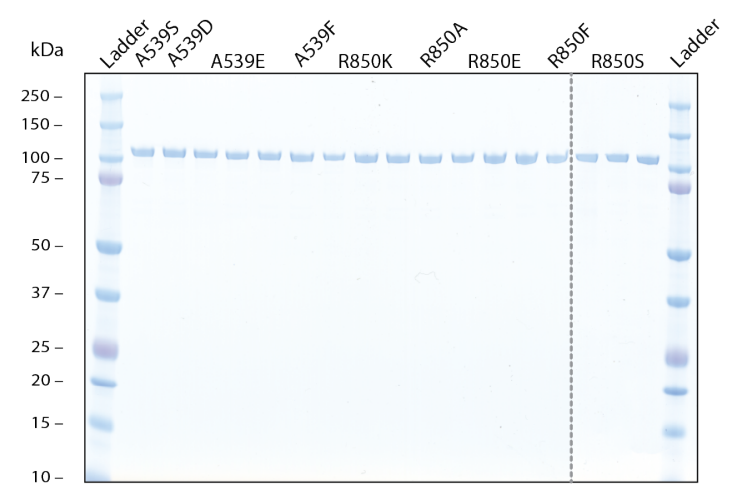

Figure S10: Analytical SDS-PAGEs (NuPAGE Bis-Tris 4-12\%) of DEBS3M5 KS ${ }^{0}$-AT mutants. Mutants A539E, R850K, R850E, and R850S expressed in biological triplicates. All AT constructs are highly pure after tandem affinity chromatography. Protein bands migrate at expected masses: A539S - 99.9kDa, A539D - 99.9kDa, A539E - 99.9kDa, A539F 99.9 kDa, R850K - 99.8 kDa, R850A - 99.8kDa, R850E - 99.8kDa, R850F - 99.8kDa, 850S - 99.8kDa, R850S - 99.8kDa. 


\section{Appendix}
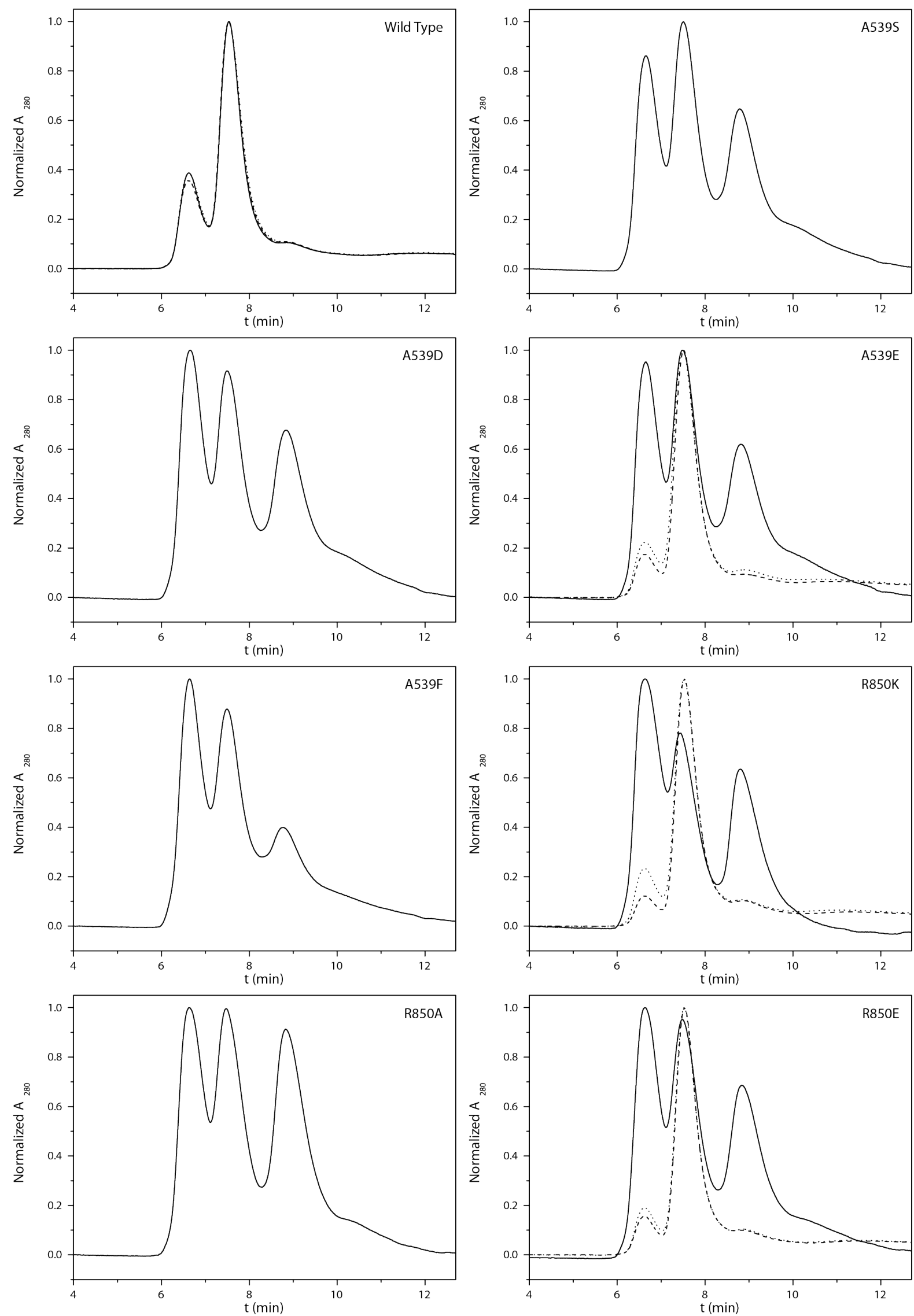

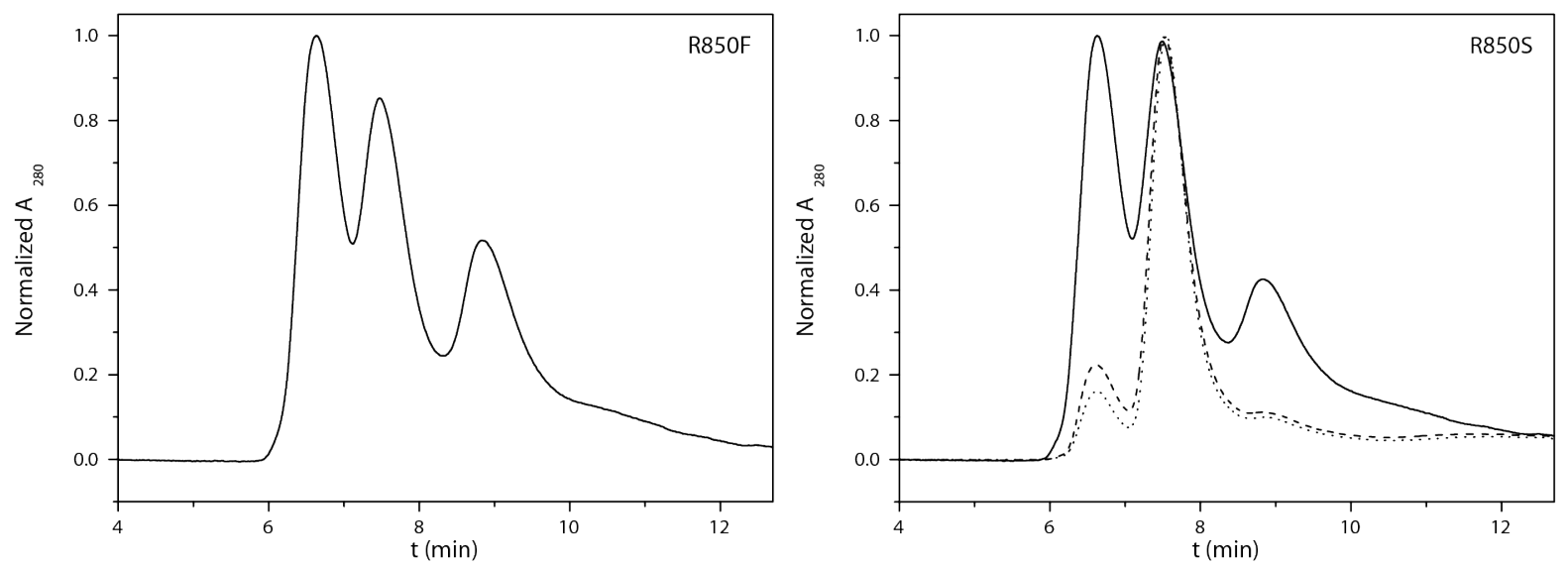

Figure S11: Normalized size exclusion chromatograms of DEBS3M5 KS ${ }^{0}$-AT mutants. Each curve (solid, dotted, dashed) corresponds to one biological replicate. All proteins show monomeric $(8.8 \mathrm{~min})$, dimeric $(7.5 \mathrm{~min})$, and tetrameric $(6.6 \mathrm{~min})$ species. 


\section{Appendix}
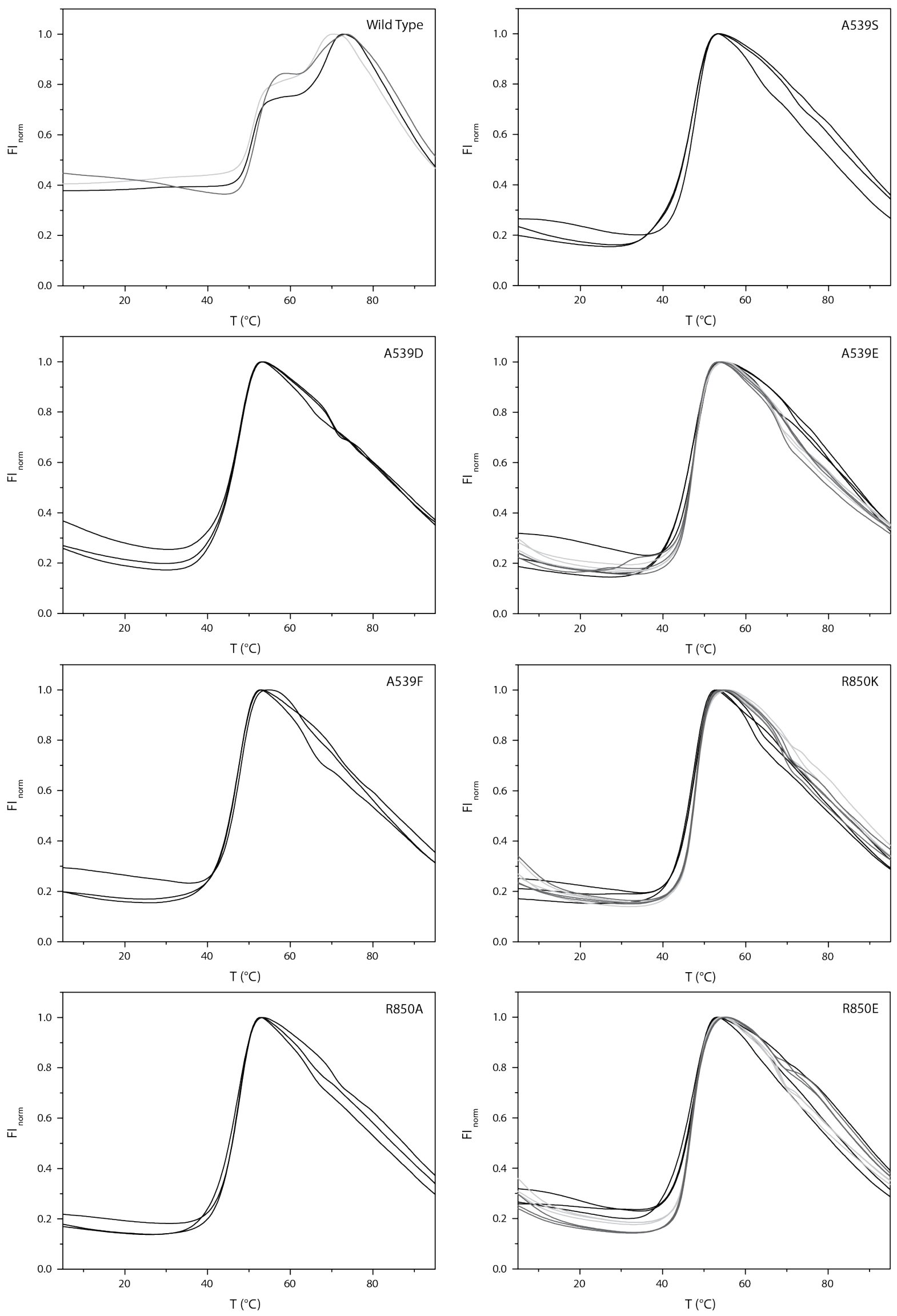

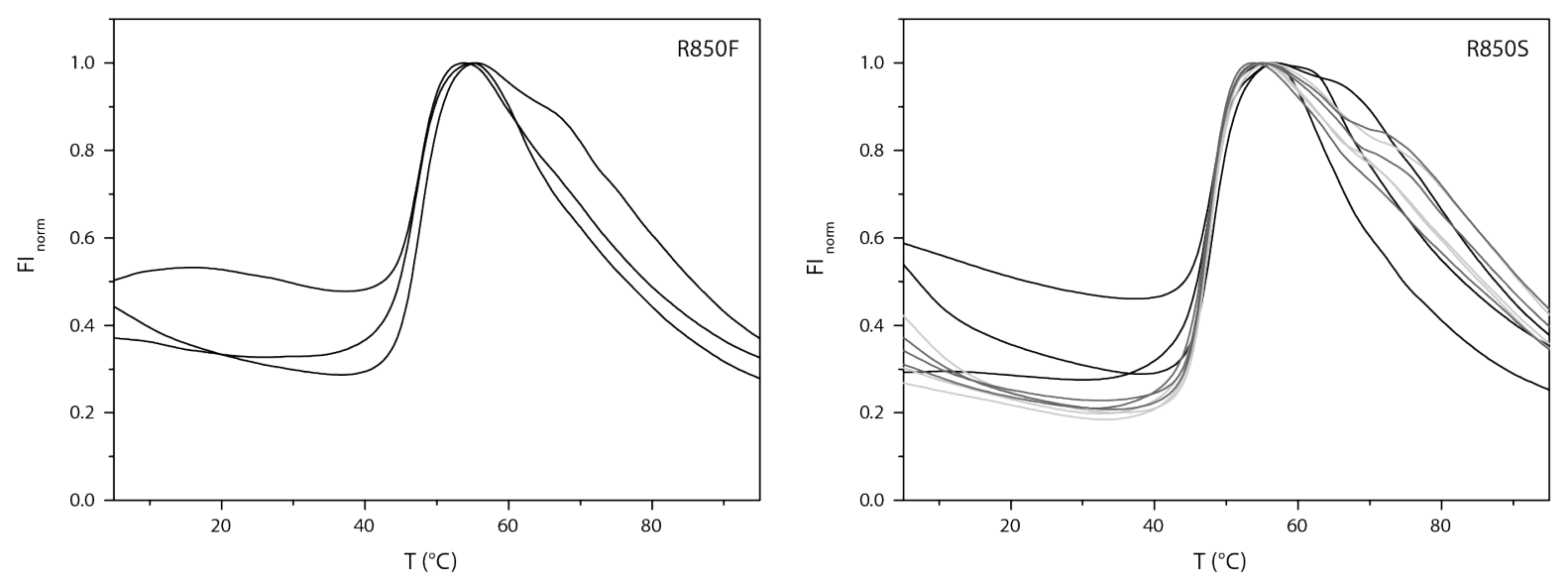

Figure S12: Normalized TSA melting curves of DEBS3M5 KS ${ }^{0}$-AT mutants. Each color (black, dark gray, and light gray) corresponds to one biological replicate. All biological replicates of mutants measured in technical triplicates in assay buffer. 


\section{Appendix}
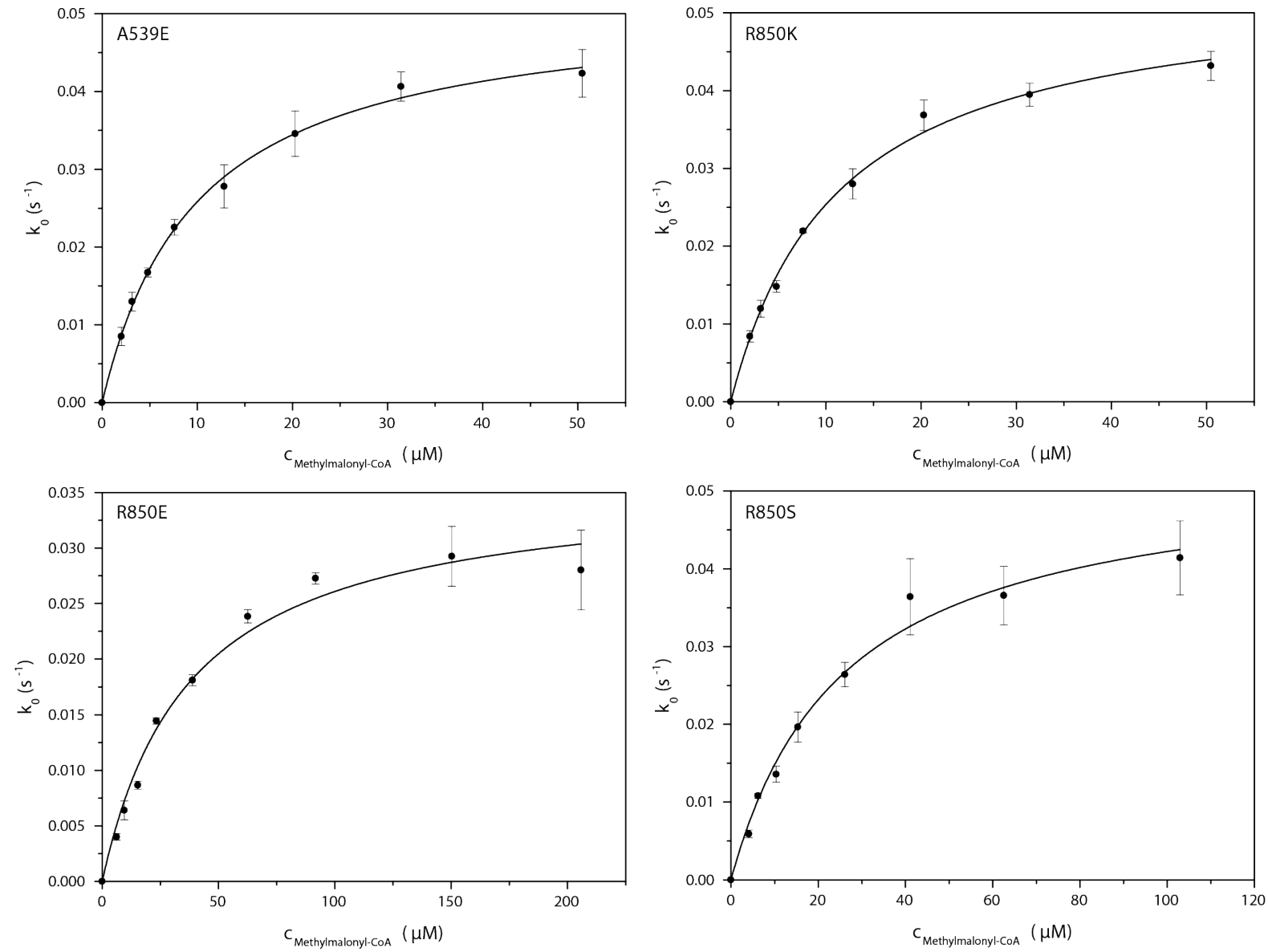

Figure S13: Titration curves for AT-mediated hydrolysis of MMal-CoA using DEBS3M5 $\mathrm{KS}^{0}$ AT mutants A539E, R850K, R850E, and R850S. Hydrolysis was measured in biological triplicates. Black dots show the average. Error bars correspond to standard deviation of biological triplicates. Kinetic parameters were determined precisely. Substrate consumption for A539E and R850K was below $10 \%$, for R850E and R850S below $5 \%$. MMal range was $0.16-5.5 \times$ $\mathrm{K}_{\mathrm{m}}^{\mathrm{MMal}-\mathrm{CoA}}$ (A539E: 0.20-5.1; R850K: 0.18-4.5; R850E: 0.16-5.5; R850S: 0.16-4.0). 

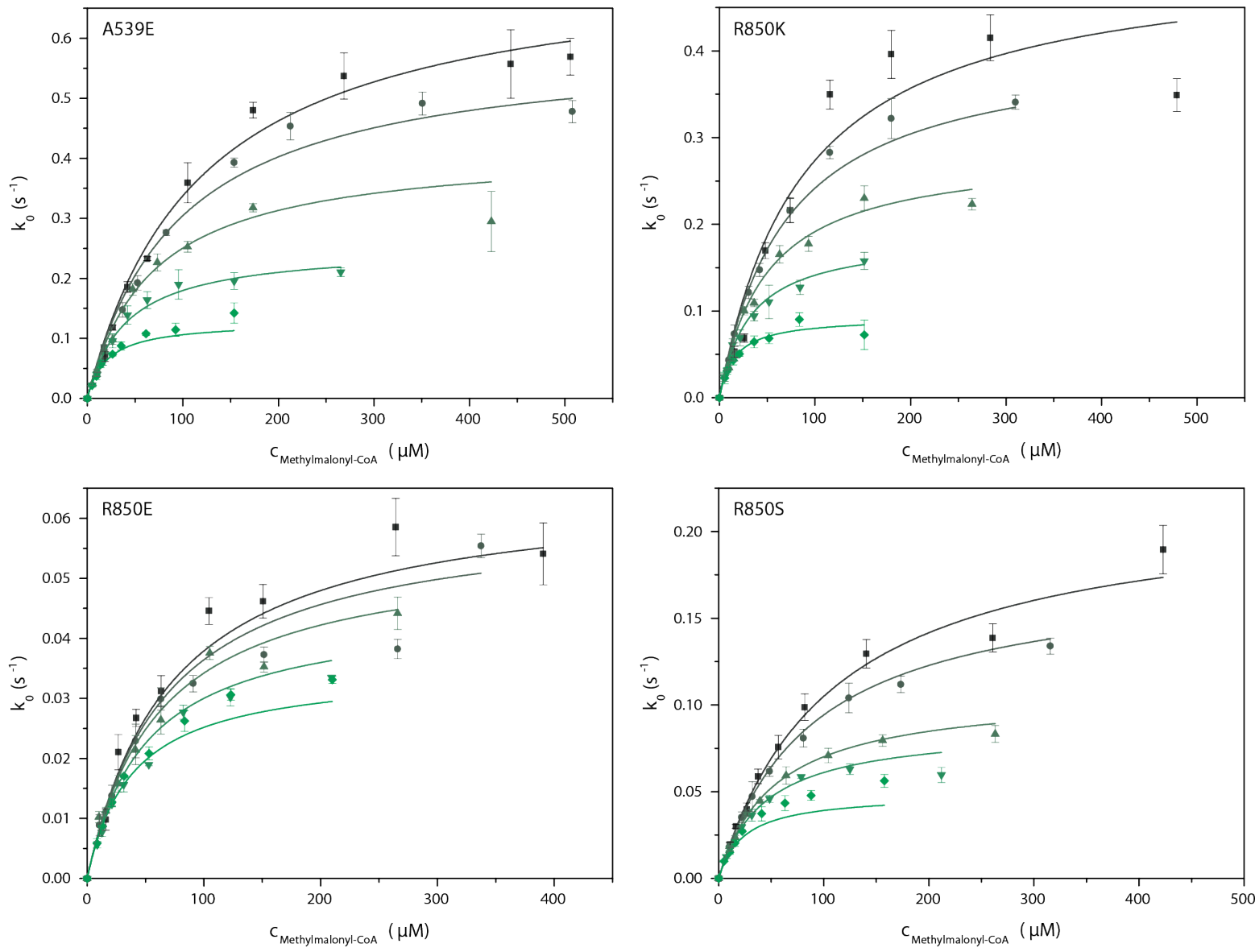

Figure S14: Global Michaelis-Menten fits of transacylation titration curves with MMal-CoA mediated by DEBS3M5 KS ${ }^{0}$-AT mutants A539E, R850K, R850E, and R850S. All measured in biological triplicates. Each color and symbol corresponds to one X-CoA titration curve at a fixed ACP concentration. Error bars give the standard deviation of biological triplicates. ACP concentrations as follows: A539E: $10.6 \mu \mathrm{M}, 24.3 \mu \mathrm{M}, 51.2 \mu \mathrm{M}, 102.3 \mu \mathrm{M}$ and $187.3 \mu \mathrm{M}, \mathrm{R} 850 \mathrm{~K}: 11.0 \mu \mathrm{M}, 26.0 \mu \mathrm{M}, 48.9 \mu \mathrm{M}, 100.4 \mu \mathrm{M}$ and $190.1 \mu \mathrm{M}, \mathrm{R} 850 \mathrm{E}: 2.5 \mu \mathrm{M}$, $4.6 \mu \mathrm{M}, 10.0 \mu \mathrm{M}, 20.3 \mu \mathrm{M}$ and $53.3 \mu \mathrm{M}$, R850S: $9.5 \mu \mathrm{M}, 20.5 \mu \mathrm{M}, 28.0 \mu \mathrm{M}, 75.5 \mu \mathrm{M}$ and 152.4 $\mu \mathrm{M}$. ACP ranges as follows: A539E: 0.14-2.5 $\times \mathrm{K}_{\mathrm{m}}^{\mathrm{ACP}}, \mathrm{R} 850 \mathrm{~K}: 0.15-2.6 \times \mathrm{K}_{\mathrm{m}}^{\mathrm{ACP}}$, R850E: $1.1-22 \times \mathrm{K}_{\mathrm{m}}^{\mathrm{ACP}}$, R850S: $0.21-3.3 \times \mathrm{K}_{\mathrm{m}}^{\mathrm{ACP}}$. Substrate consumption for R850K was below $5 \%$, for all other proteins was below $10 \%$. MMal-CoA concentration range as follows: A539E: 0.092-4.9 $\times \mathrm{K}_{\mathrm{m}}^{\mathrm{MMal} \text { app }} \mathrm{CoA}$, R850K: 0.13-9.9 $\times \mathrm{K}_{\mathrm{m}}^{\mathrm{MMal} \text { app }}$, R850E: 0.15-6.0 $\times$ $\mathrm{K}_{\mathrm{m}, \mathrm{app}}^{\mathrm{MMal}-\mathrm{CoA}}$, R850S: 0.096-6.6 $\times \mathrm{K}_{\mathrm{m} \text {, app }}^{\mathrm{MMa}-\mathrm{CoA}}$. 


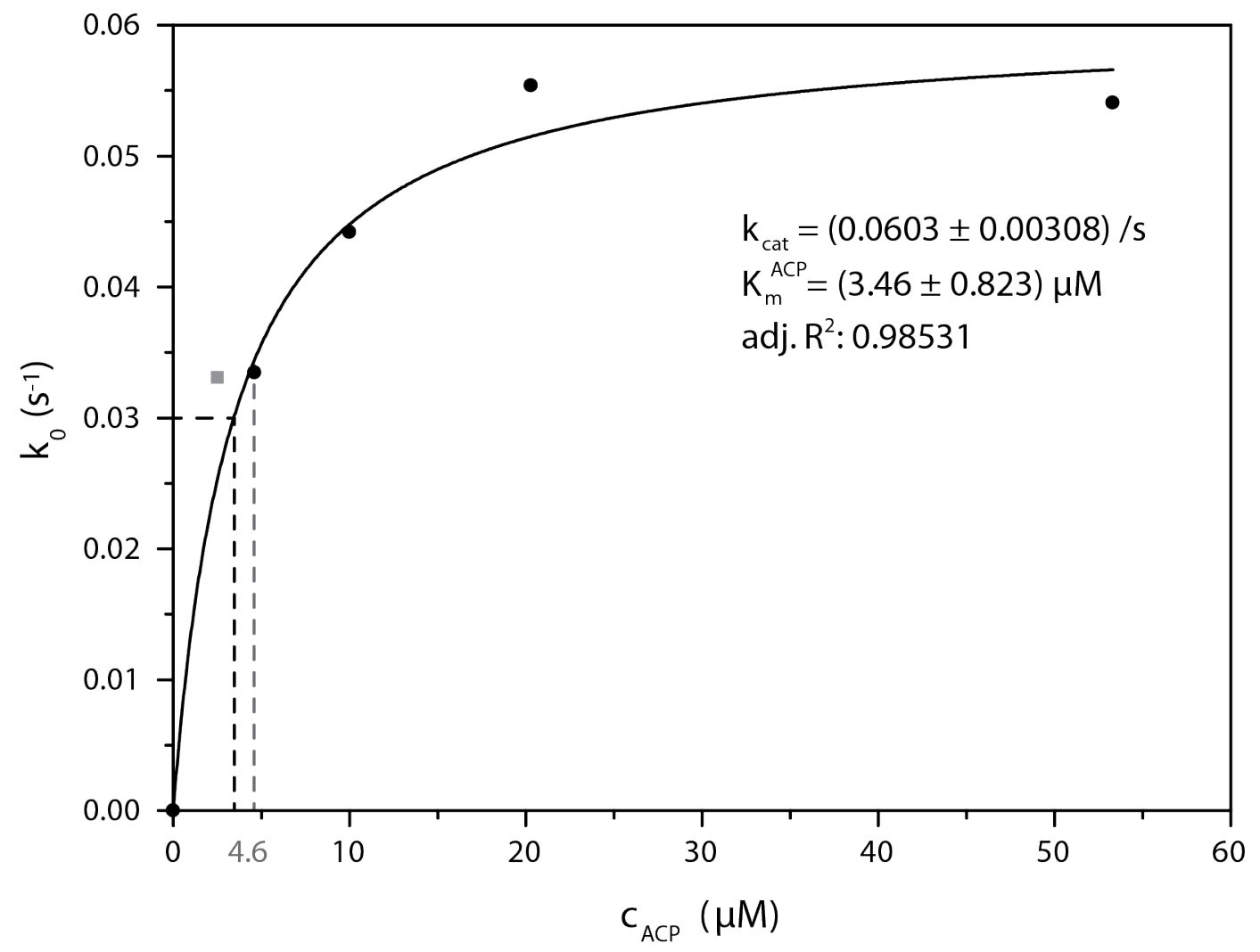

Figure S15: ACP titration curve at saturated MMal-CoA concentration of mutant R850E. Due to high measurement errors at low ACP concentrations, the kinetic parameters determined via the global fit are rather error-prone. The ACP titration curve was used to determine more reliable kinetic parameters for the AT-ACP interaction. This data was not separately determined, but data points were extracted from MMal-CoA titrations with increasing ACP concentrations (see Fig. S14). The lowest ACP concentration (gray box) is omitted to give a more accurate Michaelis-Menten fit. The new plot gives a comparable turnover rate, but a Michaelis-Menten constant of $3.46 \mu \mathrm{M}$, which is more reliable than determined before. This value is only $4.8 \%$ of the wild type $\mathrm{K}_{\mathrm{m}}^{\mathrm{ACP}}$, which is almost the same percentage as determined for the turnover rate $k_{c a t}$. The second lowest ACP concentration $(4.6 \mu \mathrm{M})$ has a $\mathrm{k}_{0}$ above half $k_{\text {cat }}$. We have taken this value as upper boundary of the Michaelis-Menten constant. 


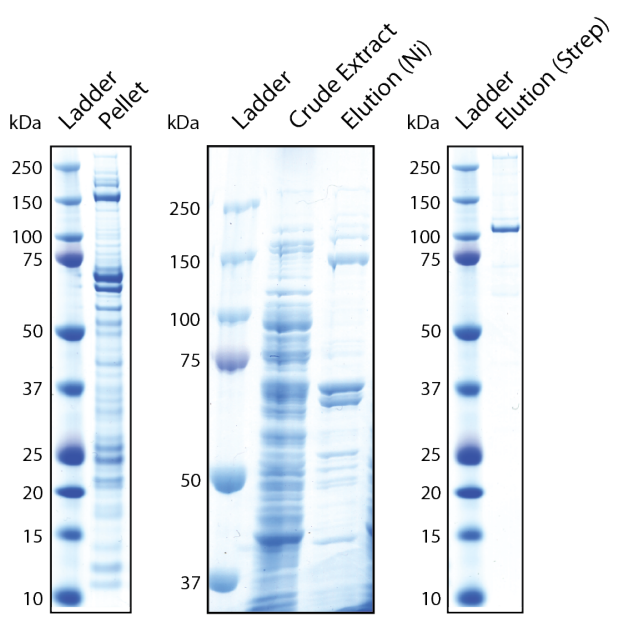

Figure S16: Purification of chimeric mFAS with DEBS3M5 AT. SDS-PAGE (NuPAGE Bis-Tris $4-12 \%$ and $7 \%$ polyacrylamide) of different purification fractions. In the elution fraction of the second affinity column (Strep), an additional protein band of around $100 \mathrm{kDa}$ was observed, which was not present in the elution fraction of the first $(\mathrm{Ni})$. This was old protein bound to the second column and most probably a KS-AT construct. 
A

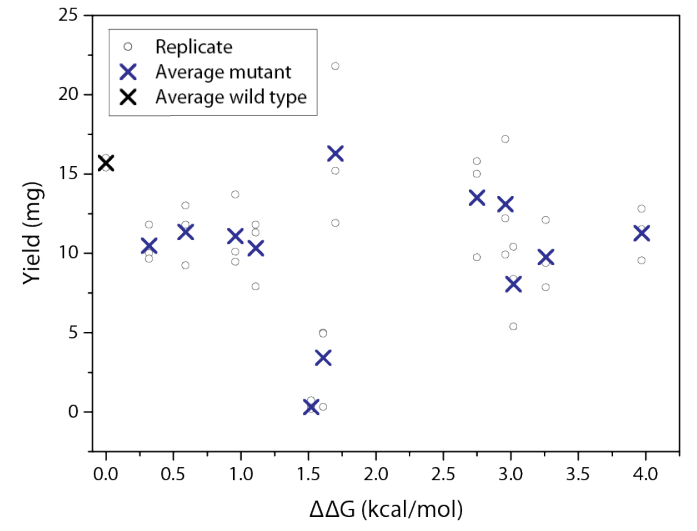

C

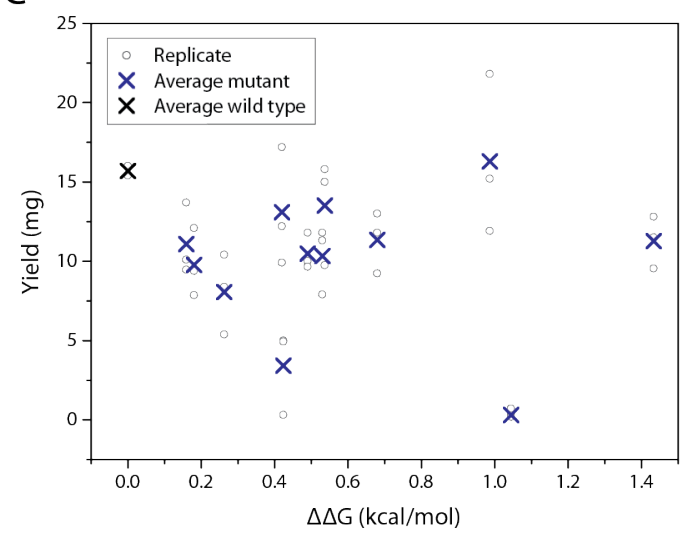

B

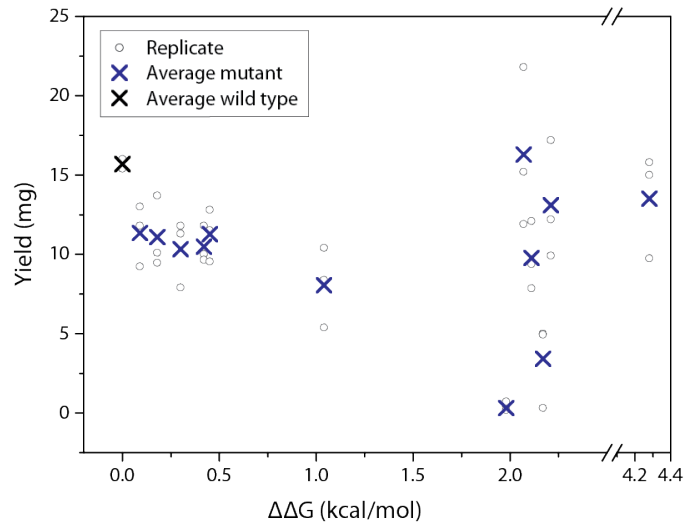

Figure S17: Yields (in $\mathrm{mg}$ per $1 \mathrm{~L}$ expression culture) of mFAS KS-AT wild type and mutants correlated to predicted changes in the Gibbs free energy $(\Delta \Delta \mathrm{G}$ in $\mathrm{kcal} / \mathrm{mol})$. (A) Chain A without PAL predicted by Robetta. (B) Chain A without PAL predicted by DrugScore. (C) Chain A with PAL predicted by $\mathrm{mCSM}$. Black circles indicate the yields of biological replicates. Average yields of biological triplicates of wild type and mutants are indicated by black and blue crosses, respectively. Please mind the scales of the different $x$-axis sectors. Predictions of the different tools showed no or only partly correlation tendencies. Overall, they were not suitable for estimating effects of mutations on protein stability. 

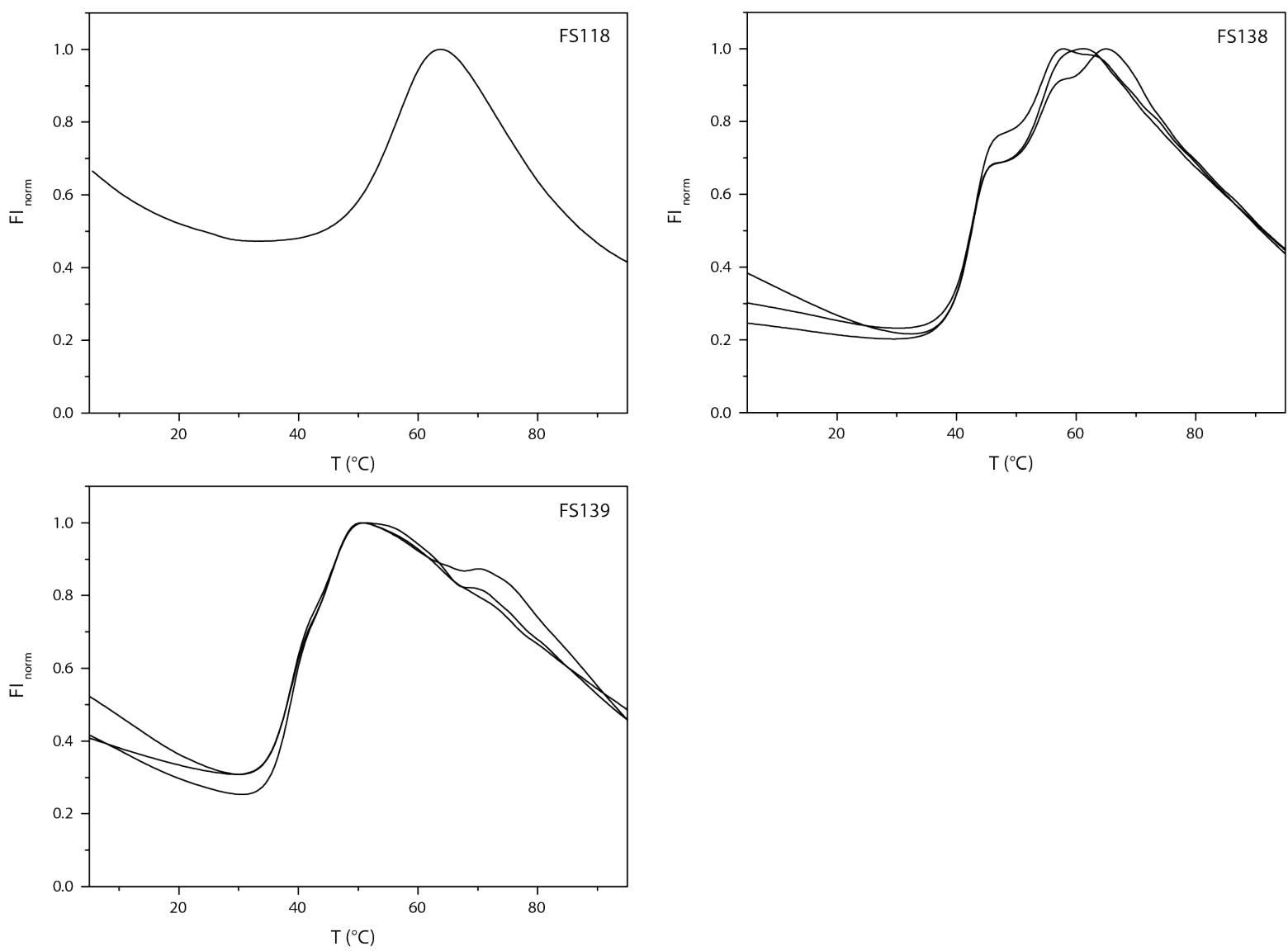

Figure S18: Normalized TSA melting curves of mFAS/DEBS3M6 KS-AT chimeras FS118 and FS139 and DEBS3M6 KS-AT FS138 in storage buffer. Each curve corresponds to one biological replicate. 


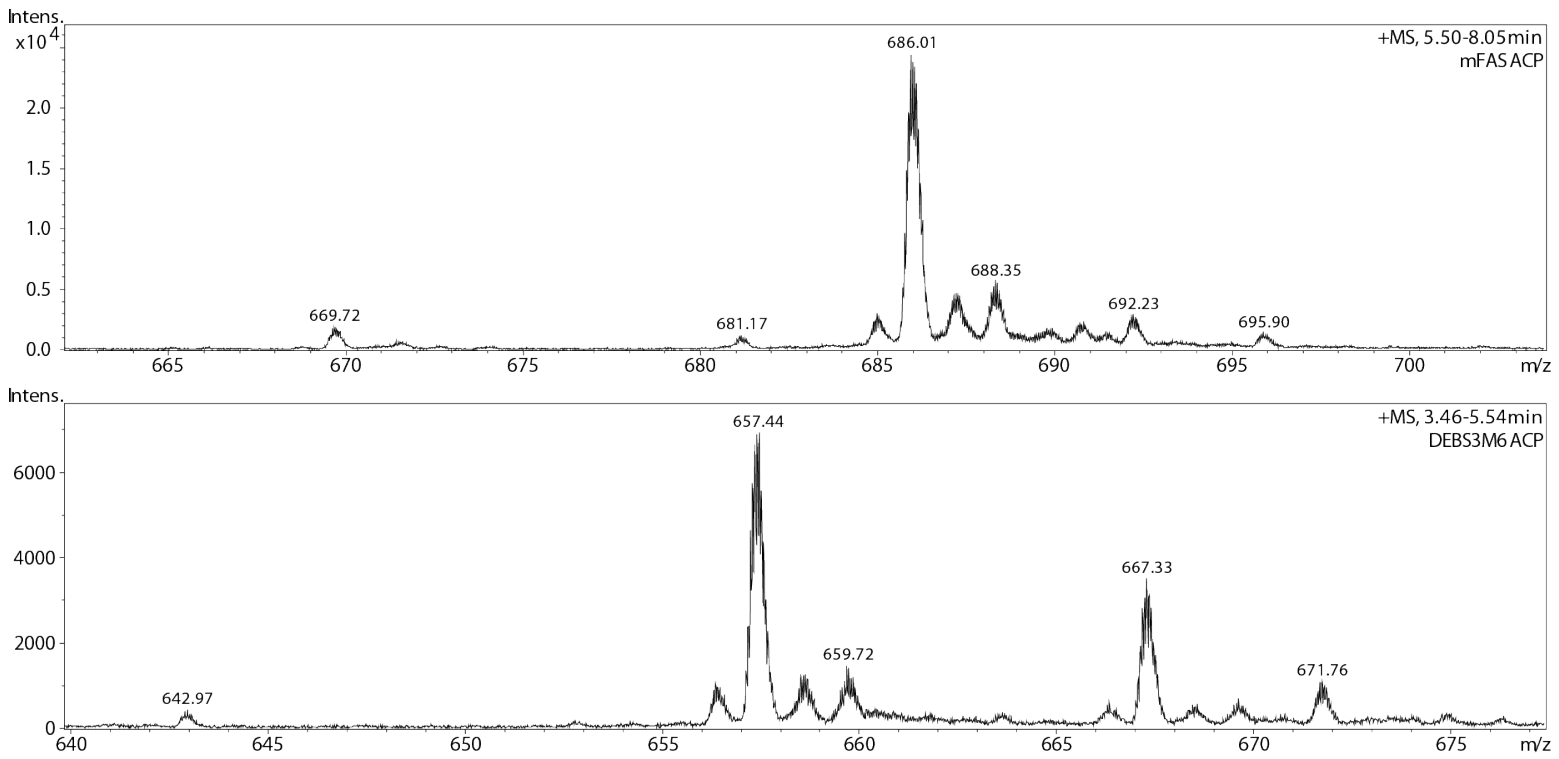

Figure S19: MS analysis of mFAS and DEBS3M6 ACP domains after SEC. Shown are the $\mathrm{m} / \mathrm{z}$-values of the average protein masses of the $18^{+}$charge state $\left(\mathrm{MS}^{1}\right)$. In none of the samples, $\mathrm{m} / \mathrm{z}$-values of the apo species were recorded. Main $\mathrm{m} / \mathrm{z}$-values correspond to the holo form. Both proteins do not include the N-terminal methionine. $\mathbf{m F A S} \mathbf{A C P}, \mathrm{z}=18 \mathrm{~m} / \mathrm{z}=$ 686.01 corresponds to holo-ACP without $\mathrm{N}$-terminal methionine. $\mathrm{m} / \mathrm{z}=688.35$ corresponds to acetylation $(\Delta \mathrm{m}=42 \mathrm{Da}), \mathrm{m} / \mathrm{z}=695.90$ corresponds to $\alpha$-N-gluconozylation $(\Delta \mathrm{m}=178 \mathrm{Da})$. $\mathrm{m} / \mathrm{z}=692.23$ is an unknown modified variant $(\Delta \mathrm{m}=112 \mathrm{Da}) . \mathrm{m} / \mathrm{z}=681.17$ corresponds to holo-ACP without $\mathrm{N}$-terminal methionine and serine $(\Delta \mathrm{m}=-87 \mathrm{Da})$. DEBS3M6 ACP, $\mathrm{z}=18: \mathrm{m} / \mathrm{z}=657.44$ corresponds to holo-ACP without $\mathrm{N}$-terminal methionine. $\mathrm{m} / \mathrm{z}=$ 659.72 corresponds to acetylation $(\Delta \mathrm{m}=41 \mathrm{Da}), \mathrm{m} / \mathrm{z}=667.33$ and 671.76 correspond to $\alpha$-N-gluconozylation $(\Delta \mathrm{m}=178 \mathrm{Da}$ and $258 \mathrm{Da}) . \mathrm{m} / \mathrm{z}=692.23$ is an unknown modified variant $(\Delta \mathrm{m}=112 \mathrm{Da})$. 
A

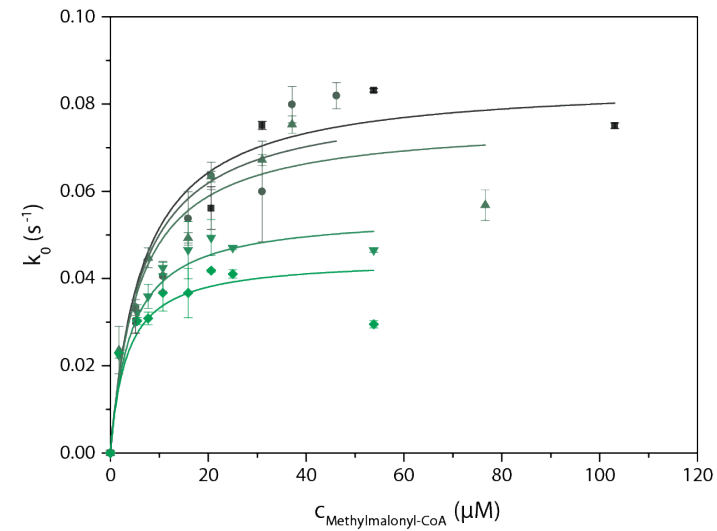

C

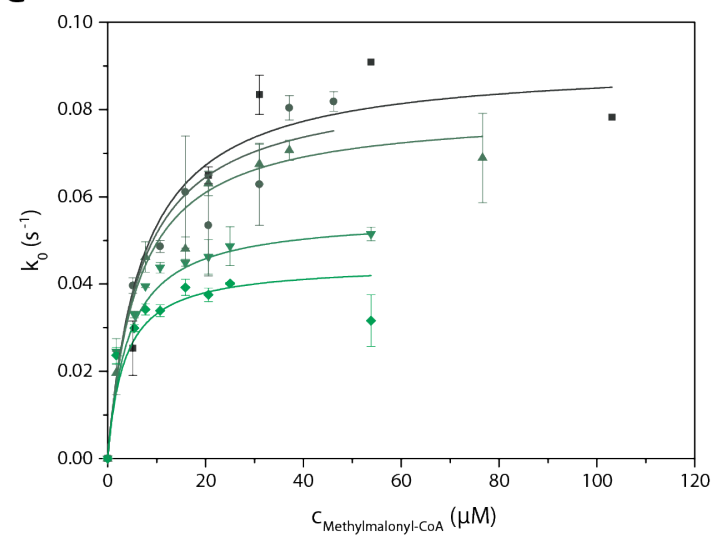

B

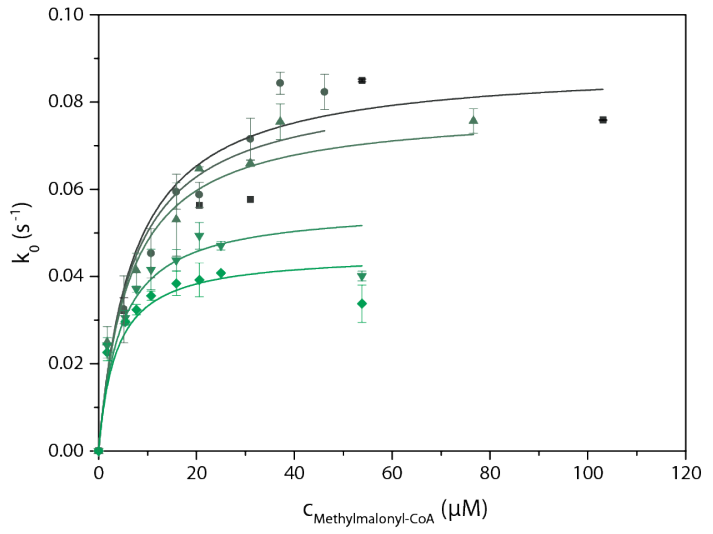

D

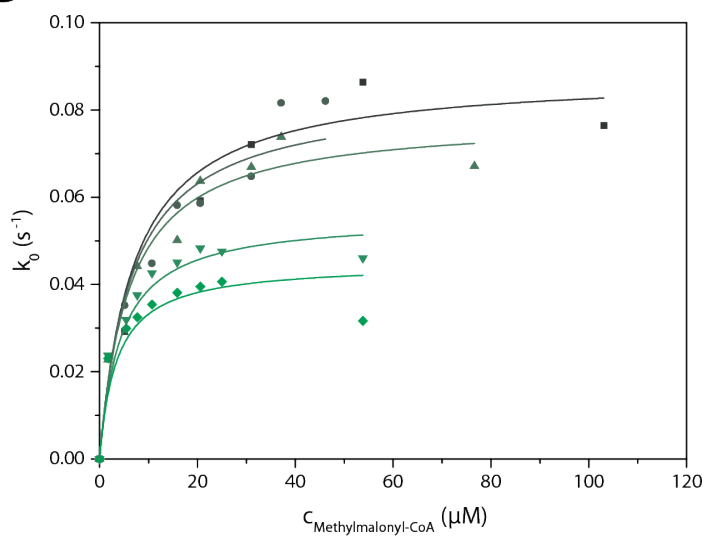

Figure S20: Global Michaelis-Menten fits of transacylation titration curves with MMal-CoA mediated by $\mathrm{m}(\mathrm{KS})$-DEBS3M6(AT) chimera FS118. (A)-(C) Biological replicates measured in technical triplicates. Each color and symbol corresponds to one X-CoA titration curve at a fixed ACP concentration. Error bars give the standard deviation of the technical triplicates. (D) Average of biological replicates. Each color and symbol corresponds to one X-CoA titration curve at a fixed ACP concentration. ACP concentrations as follows: $67.1 \mu \mathrm{M}, 98.4 \mu \mathrm{M}$, $227.3 \mu \mathrm{M}, 290.0 \mu \mathrm{M}$ and $360.9 \mu \mathrm{M}$. ACP range as follows: $0.65-3.5 \times \mathrm{K}_{\mathrm{m}}^{\mathrm{ACP}}$. Substrate consumption was up to $26 \%$. MMal-CoA concentration range as follows: $0.17-85 \times \mathrm{K}_{\mathrm{m} \text {, app }}^{\mathrm{MMa} \text { a } \mathrm{Co}}$. 


\section{Appendix}

A

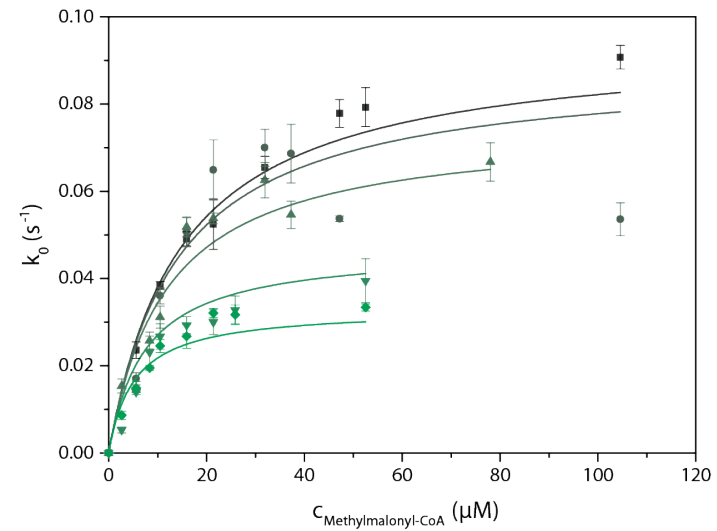

C

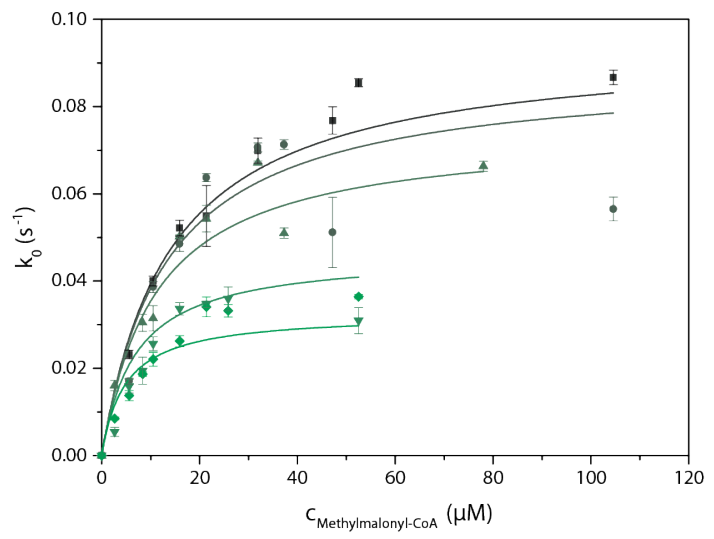

B

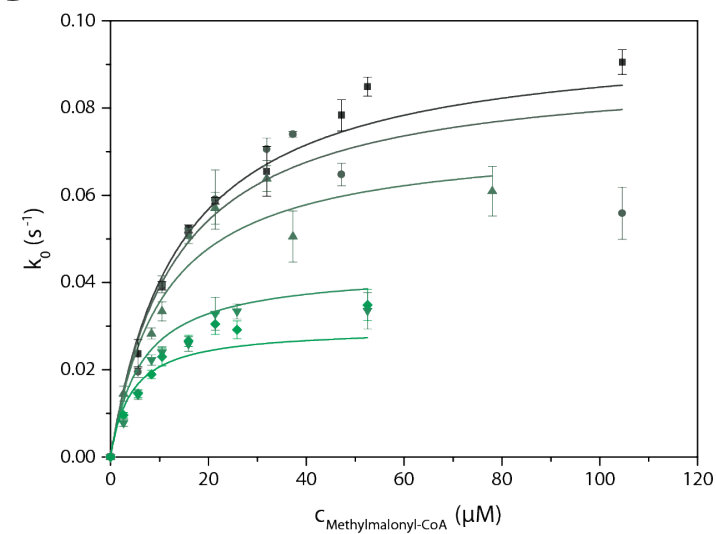

D

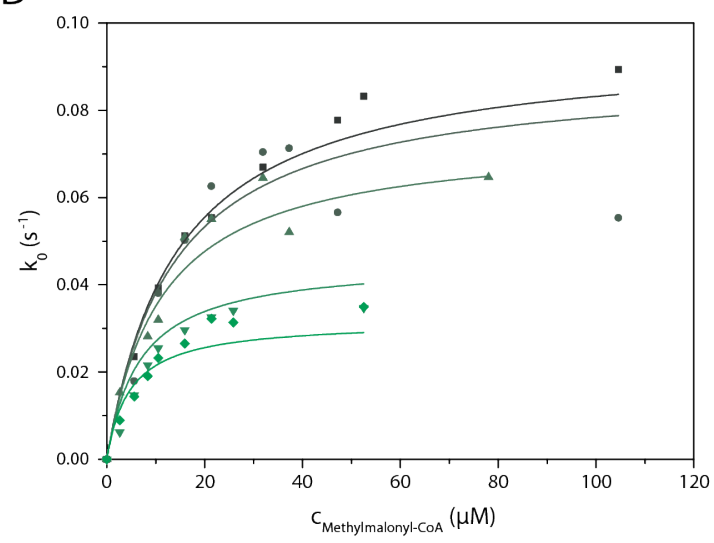

Figure S21: Global Michaelis-Menten fits of transacylation titration curves with MMal-CoA mediated by DEBS3M6(KS-AT) wild type FS138. (A)-(C) Biological replicates measured in technical triplicates. Each color and symbol corresponds to one X-CoA titration curve at a fixed ACP concentration. Error bars give the standard deviation of the technical triplicates. (D) Average of biological replicates. Each color and symbol corresponds to one X-CoA titration curve at a fixed ACP concentration. ACP concentrations as follows: $61.5 \mu \mathrm{M}, 98.4 \mu \mathrm{M}$, $211.9 \mu \mathrm{M}, 303.5 \mu \mathrm{M}$ and $354.0 \mu \mathrm{M}$. ACP range as follows: $0.24-1.4 \times \mathrm{K}_{\mathrm{m}}^{\mathrm{ACP}}$. Substrate consumption was up to $24 \%$. MMal-CoA concentration range as follows: $0.14-11 \times \mathrm{K}_{\mathrm{m}}^{\mathrm{MMal} \text { app }}$. 


\section{Appendix}

A

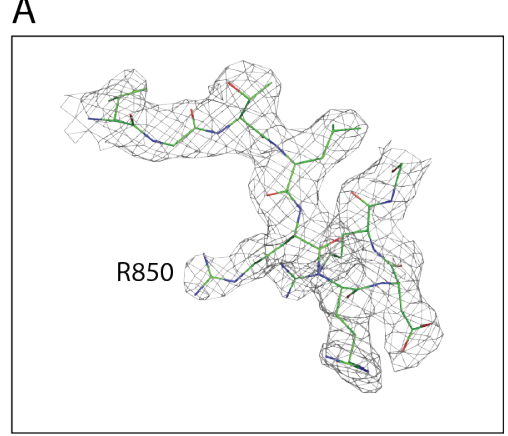

D

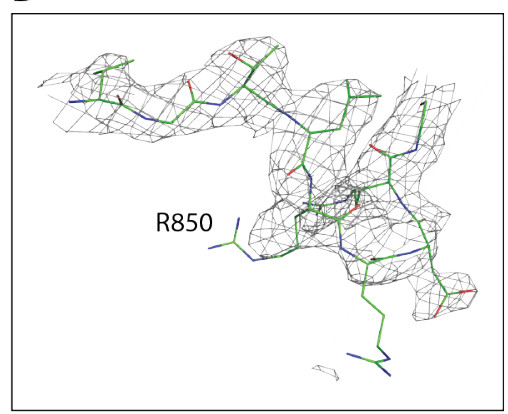

B

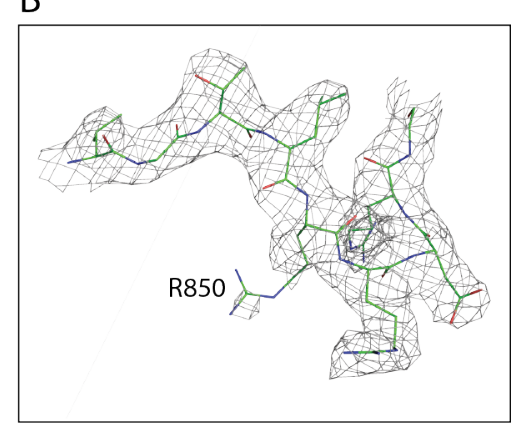

E

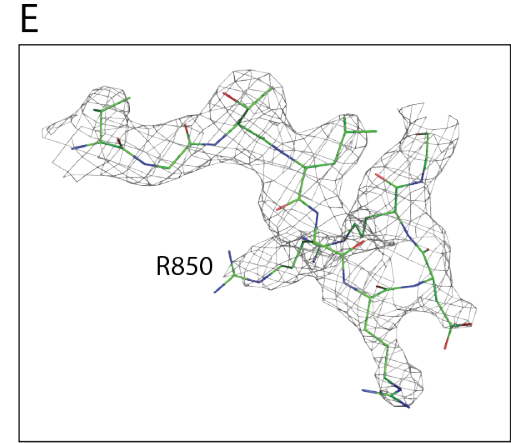

C

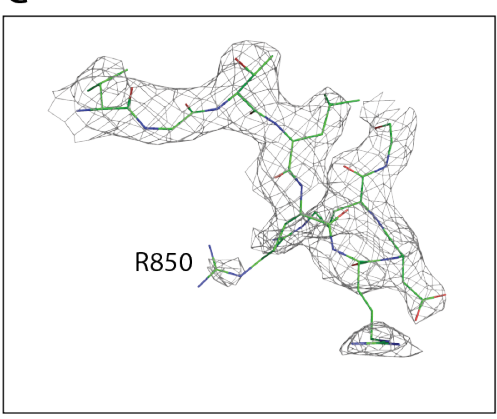

$\mathrm{F}$

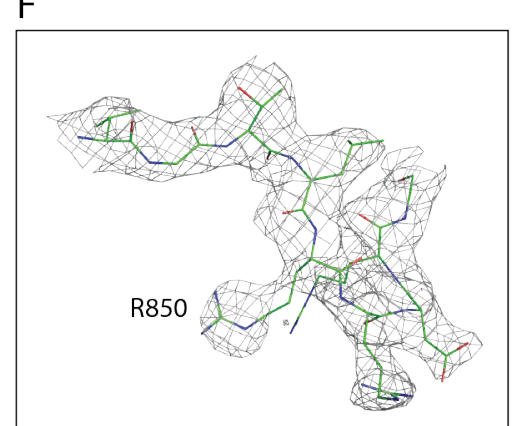

Figure S22: Electron density map of residue R850. The electron density map for the residues V846-G854 of DEBS3M5 AT (PDB: $2 \mathrm{HG}^{[9]}$ ) for all six chains (A-F) shows poor electron density for the interfacial residue R850 mutated in this study. This finding supports the flexibility of this residue. Different binding modes might be involved during catalysis. The 2fo-fc map was created using PyMOL (MacPyMOL Version 1.7.0.3, Schrödinger, LLC) $($ sigma $=1$, carve $=1.8)$. 


\section{Appendix}

A

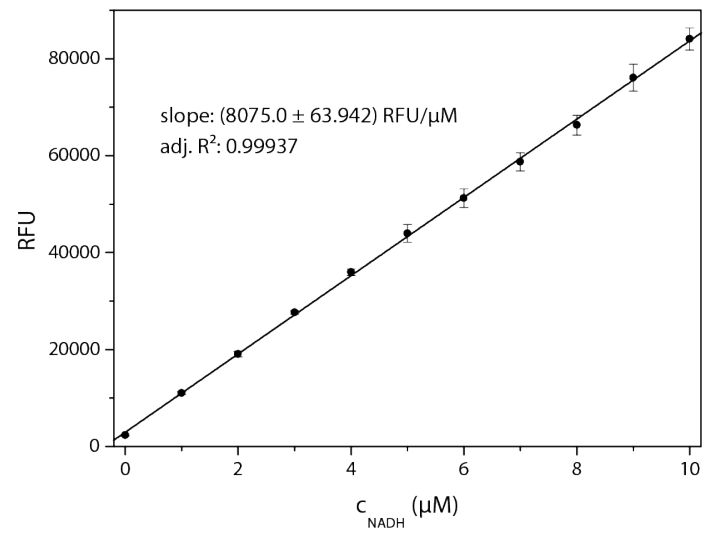

B

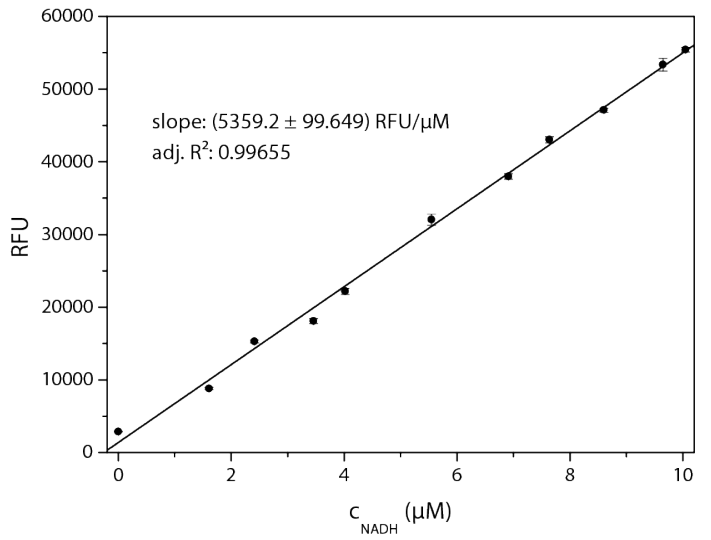

Figure S23: NADH calibration. For quantification of AT activity, an NADH calibration was performed. (A) 96-well format. 8075 relative fluorescence units (RFU) correspond to $1 \mu \mathrm{M}$ $\mathrm{NADH}$. The NADH calibration factor changes over time due to the decreasing power of the UV lamp. An internal standard ( $50 \mu \mathrm{M}$ X-CoA and $50 \mu \mathrm{M}$ ACP) was measured the same day as the NADH calibration. This internal standard was used to determine the calibration factor for each measurement time span (Tab.S11). This factor was subsequently used to convert RFU into concentrations $(\mu \mathrm{M})$ and ranges from 13450 to 8075 RFU per $1 \mu \mathrm{M}$ NADH. Error bars represent the standard deviation of three independent measurements that were measured in technical triplicates. (B) 384-well format. 5359 RFU correspond to $1 \mu \mathrm{M} \mathrm{NADH}$. Error bars represent the standard deviation of technical triplicates. 


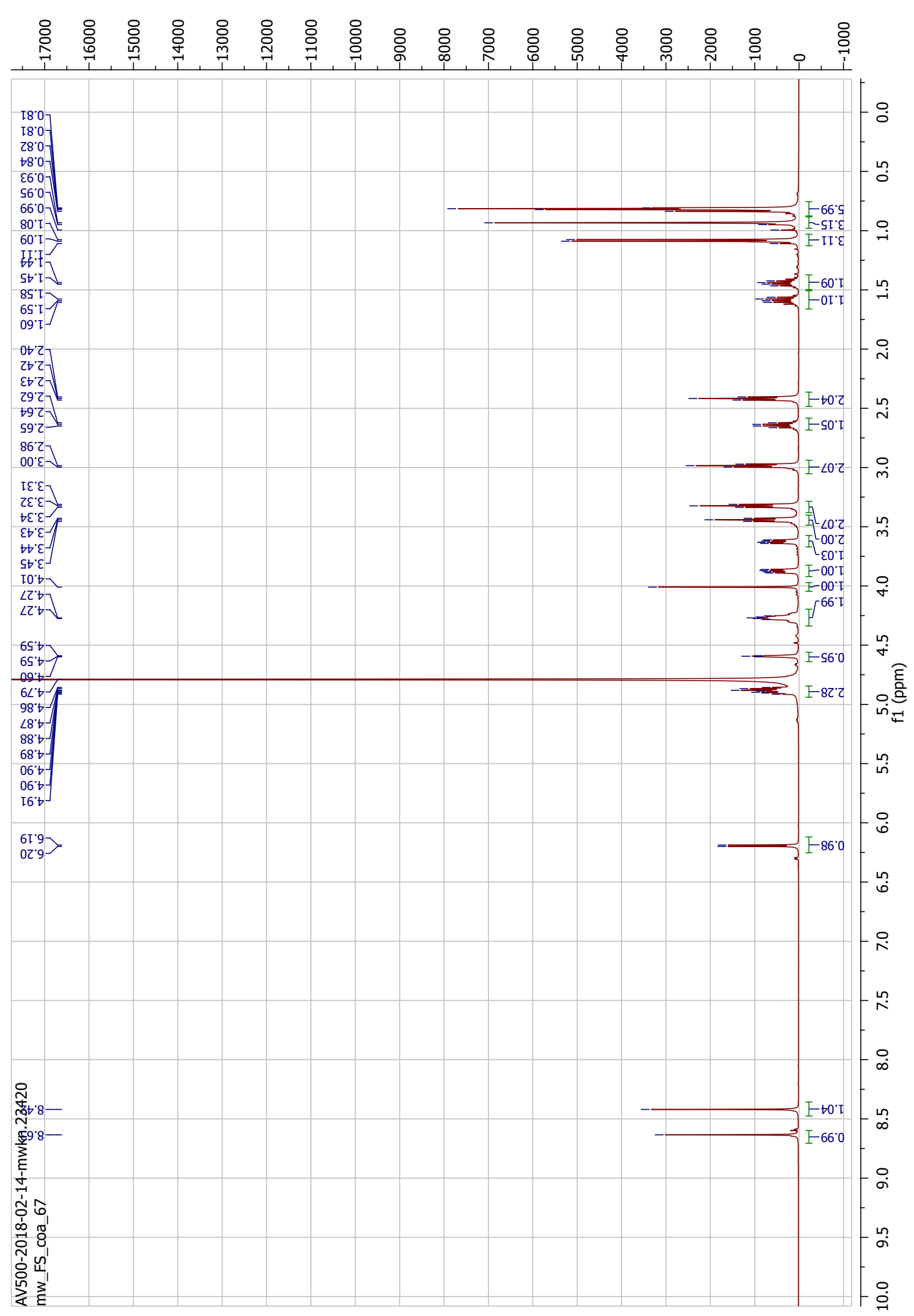

Figure S24: ${ }^{1} \mathrm{H}-\mathrm{NMR}$ of 2-methylbutyryl-CoA. 


\section{Statement of Personal Contributions}

Except where stated otherwise by reference or acknowledgment, the work presented in this thesis was generated by myself. Projects were regularly discussed with my advisor Prof. Dr. Martin Grininger (Goethe-University Frankfurt, Institute for Organic Chemistry and Chemical Biology). Contributions from colleagues and students are explicitly referenced at respective parts in the thesis. A detailed statement of personal contributions is given below.

The following persons contributed to the results of this thesis:

Dr. Christina S. Heil (AK Grininger, Goethe-University Frankfurt, Institute for Organic Chemistry and Chemical Biology) and Dr. Alexander Rittner (AK Grininger, Goethe-University Frankfurt, Institute for Organic Chemistry and Chemical Biology) contributed through their supervision during my master's thesis and conceived together with Prof. Dr. Martin Grininger (Goethe-University Frankfurt, Institute for Organic Chemistry and Chemical Biology) the initial idea of the kinetic characterization of AT domains.

Dr. Alexander Rittner (AK Grininger, Goethe-University Frankfurt, Institute for Organic Chemistry and Chemical Biology) helped choosing the first domain boundaries for AT swapping.

My colleague Lynn Buyachuihan (AK Grininger, Goethe-University Frankfurt, Institute for Organic Chemistry and Chemical Biology) was involved in the project of creating mFAS-based elongation modules. We conceived this project together with Prof. Dr. Martin Grininger (Goethe-University Frankfurt, Institute for Organic Chemistry and Chemical Biology). Lynn Buyachuihan was involved in isolation of the elongation modules. She performed the TSA, the CoA-488 assay, activity assays, and LC-MS analyses of all modules.

Student Maren Berlinghof was involved in the construction of chimeric mFAS/DEBS3M5 KS-AT as well as in the isolation and analysis of mFAS and DEBS3M5 KS-AT wild types during her master's thesis under my supervision. Furthermore, she assisted in the isolation of AT domains for their kinetic characterization.

Student Alicia Just was involved in isolation and analysis of mFAS/DEBS3M6 KS-AT constructs during her master's thesis under my supervision. 
Students Guanzhu Götze, Shengnan Li, and Yunqing Li assisted in cloning plasmids encoding the DEBS3M5 AT:ACP interface mutants.

Student Amin Fahim assisted in the DEBS3M5 AT:ACP interface mutation study during his bachelor's thesis under my supervision.

Student Robin Klimek assisted in the isolation of AT domains for their kinetic characterization.

Student Tanja Ott was involved in the construction of chimeric mFAS-based elongation modules with VemG, VemH, and EcPKS1 AT.

Student Matthias Zeug was involved in isolation and analysis of mFAS-based elongation modules during his master's thesis under supervision of Lynn Buyachuihan (AK Grininger, Goethe-University Frankfurt, Institute for Organic Chemistry and Chemical Biology).

Student Laurell Kessler was involved in analysis of mFAS-based elongation modules under supervision of Lynn Buyachuihan (AK Grininger, Goethe-University Frankfurt, Institute for Organic Chemistry and Chemical Biology).

Dr. Gina Grammbitter (AK Bode, Goethe-University Frankfurt, Institute for Molecular Bio Science) kindly performed HPLC-MS analyses of ACPs and assisted with analysis of the data.

Dr. Karthik S. Paithankar (AK Grininger, Goethe-University Frankfurt, Institute for Organic Chemistry and Chemical Biology) performed the Rosetta calculations of the mFAS and DEBS3M5 KS-AT wild types. Furthermore, he was involved in the crystallization experiments.

Dr. Apirat Chaikuad (AK Knapp, Goethe-University Frankfurt, Institute for Pharmaceutical Chemistry) was involed in the crystallization experiments.

Marek Wanior (AK Knapp, Goethe-University Frankfurt, Institute for Pharmaceutical Chemistry) kindly assisted in purification of 2-MB-CoA via HPLC.

Alexander Stegemann (AK Rischke, Goethe-University Frankfurt, Institute for Theoretical Physics) kindly discussed the derivation of kinetic parameters and assisted in the mathematical examination of changes in transacylation kinetic parameters caused by point mutations.

Martin Schwalm (AK Knapp, Goethe-University Frankfurt, Institute for Pharmaceutical Chemistry) assisted with depiction of electron density data. 


\section{Appendix}

\section{Copyright and Creative Commons Licences}

Whenever a figure, table, or text is identical to a previous publication, it is hereby stated explicitly that copyright permission and/or co-author agreement has been obtained.

The following figures of the thesis have been modified from previous publications:

- Fig. 2.5 "Structure and domain organization of typel FAS systems.": Heil, Christina S., Wehrheim, S. Sophia, Paithankar, Karthik S., Grininger, Martin "Fatty Acid Biosynthesis: Chain-Length Regulation and Control" ChemBioChem 20, 2298-2321 (2019). [41] Copyright Wiley-VCH GmbH. Reproduced with permission.

- Fig. 2.6 "Structural models for modular PKSs.": Reproduced from Klaus, Maja and Grininger, Martin "Engineering strategies for rational polyketide synthase" Natural Product Reports 35, 1070-1081 (2018) ${ }^{[75]}$ with permission from the Royal Society of Chemistry.

- Fig. 2.8 "Reaction catalyzed by the AT.": Heil, Christina S., Wehrheim, S. Sophia, Paithankar, Karthik S., Grininger, Martin "Fatty Acid Biosynthesis: Chain-Length Regulation and Control" ChemBioChem 20, 2298-2321 (2019). [41] Copyright Wiley-VCH GmbH. Reproduced with permission.

- Fig. 2.9 "Reaction catalyzed by the KS.": Heil, Christina S., Wehrheim, S. Sophia, Paithankar, Karthik S., Grininger, Martin "Fatty Acid Biosynthesis: Chain-Length Regulation and Control" ChemBioChem 20, 2298-2321 (2019). [41] Copyright Wiley-VCH GmbH. Reproduced with permission.

The following parts of the thesis have been (in parts) previously published: Stegemann, Franziska and Grininger, Martin "Transacylation Kinetics in Fatty Acid and Polyketide Synthases and its Sensitivity to Point Mutations" ChemCatChem (2021). ${ }^{[1]}$

- Chapters/Sections:

1 "Abstract"

3.2 "Kinetic Description of AT-mediated Reactions"

3.3 "Kinetic Analysis of Acyl Transferases"

4.2 "Kinetic Analysis of Acyl Transferases"

4.3 "Implications of Kinetic Properties on the AT Reaction Mechanism"

4.4 "Implications of Kinetic Properties on FAS/PKS Function and Evolution"

4.5 "Impact of Interface Mutations on Transacylation Kinetics"

4.6 "Implications of Interface Mutation Study on PKS Engineering"

4.11 "Conclusion and Outlook"

5 "Experimental Procedures" 


\section{- Figures:}

Fig. 2.4 "Phylogenetic relationship between FASs and PKSs."

Fig. 3.1 "Scheme of AT-mediated reactions."

Fig. 3.2 "Phylogenetic tree of AT domains."

Fig. 3.4 "Transacylation and hydrolysis screening of FAS and PKS ATs."

Fig. 3.5 "Transacylation parameters of FAS and PKS ATs."

Fig. 3.7 "Transacylation and hydrolysis screening of DEBS M5 AT:ACP interface mutants."

Fig. 3.8 "Transacylation parameters of DEBS M5 AT:ACP interface mutants."

Fig. 3.9 "Transacylation transition state energy of DEBS M5 AT:ACP interface mutants."

Fig. 4.1 "Plasticity of transacylation kinetics of DEBS M5 AT:ACP interface mutants."

Fig. S1 "SDS-PAGEs of FAS and PKS ATs."

Fig. S2 "Size exclusion chromatograms of FAS and PKS ATs."

Fig. S3 "TSA melting curves of FAS and PKS ATs."

Fig. S4 "SDS-PAGEs of FAS and PKS ACPs."

Fig. S5 "Mass spectrometric analysis of FAS and PKS ACPs."

Fig. S6 "TSA melting curves of FAS and PKS ACPs."

Fig. S7 "Titration curves for AT-mediated hydrolysis."

Fig. S8 "Titration curves for AT-mediated transacylation."

Fig. S9 "Detailed model of DEBS M5 AT:ACP interface."

Fig. S10 "SDS-PAGEs of DEBS M5 AT:ACP interface mutants."

Fig. S11 "Size exclusion chromatograms of DEBS M5 AT:ACP interface mutants."

Fig. S12 "TSA melting curves of DEBS M5 AT:ACP interface mutants."

Fig. S13 "Hydrolysis curves for DEBS M5 AT:ACP interface mutants."

Fig. S14 "Transacylation curves for DEBS M5 AT:ACP interface mutants."

Fig. S15 "ACP titration curve of DEBS M5 AT:ACP mutant R850E."

Fig. S22 "Electron density map of DEBS M5 AT residue R850."

Fig. S23 "NADH calibration." 


\section{Appendix}

- Tables:

Tab. 3.1 "Hydrolysis parameters of FAS and PKS ATs."

Tab. 3.2 "Transacylation parameters of FAS and PKS ATs."

Tab. 3.3 "Kinetic parameters of DEBS M5 AT:ACP interface mutants."

Tab. S1 "Expression yields of FAS and PKS ATs."

Tab. S2 "TSA of FAS and PKS ATs and ACPs."

Tab. S3 "Expression yields of FAS and PKS ACPs."

Tab. S4 "Mass spectrometric analysis of FAS and PKS ACPs."

Tab. S5 "Initial substrate screening of FAS and PKS ATs."

Tab. S6 "Hydrolysis parameters of FAS and PKS ATs after transacylation."

Tab. S7 "Expression yields of DEBS M5 AT:ACP interface mutants."

Tab. S8 "TSA of DEBS M5 AT:ACP interface mutants."

The complete draft of the manuscript accepted for publication as Stegemann, Franziska and Grininger, Martin "Transacylation Kinetics in Fatty Acid and Polyketide Synthases and its Sensitivity to Point Mutations" ChemCatChem (2021) was initially written by myself. Prof. Dr. Martin Grininger participated significantly with editing and phrasing to the final version in the process of publication. Copyright retains by the authors. Creative Commons Attribution 4.0 International License (CC BY 4.0).

\section{Copyright and Creative Commons declaration of the ChemCatChem journal:}

"Authors retain copyright of their article and are given a choice of Creative Commons license under which to publish their work."

Source:

https://onlinelibrary.wiley.com/page/journal/18673899/homepage/2491_onlineopen.html (accessed 29th April 2021) 
The following master's and bachelor's theses, which were submitted to the department 14 - Biochemistry, Chemistry and Pharmacy of the Goethe-University Frankfurt, contributed to the results of the present thesis:

Franziska Stegemann "Fluorescence Studies on Dynamic Domains of the Mammalian Fatty Acid Synthase and Different Polyketide Synthases" Master's thesis (2016). [210]

Maren Julia Berlinghof "Analyse von Domänen-Domänen-Interaktionen in Megasynthasen" Master's thesis (2018). [223]

Amin Fahim "Modulation einer AT:ACP-Interaktionsfläche der 6-Desoxyerythronolid BSynthase" Bachelor's thesis (2019). [256]

Alicia Just "Analyse von Fettsäure- und Polyketidsynthase-Hybriden in Struktur und Funktion" Master's thesis (2020). [226]

The following figures were used in a modified way, prepared by me, and printed with my permission in Maren Berlinghof's master's thesis. ${ }^{\text {[223] }}$

- Fig. 3.22 "Correlation of Rosetta predictions with mFAS KS-AT yields."

- Fig. 3.23 "Correlation of Rosetta predictions with mFAS KS-AT melting temperatures."

- Fig. 3.25 "Correlation of Rosetta predictions with DEBS M5 KS-AT yields."

The following figures, prepared by me, were used in a modified way, prepared by Maren Berlinghof, in her master's thesis. [223]

- Fig. 3.14 "Analysis of mFAS/DEBS M5 KS-AT chimeras."

- Fig. 3.17 "TSA of mFAS KS-AT mutants."

- Fig. 3.18 "HPLC-SEC of mFAS KS-AT mutants."

- Fig. 3.24 "Correlation of Rosetta predictions with mFAS KS-AT oligomeric states."

- Fig. S17 "Correlation of bioinformatics tools predictions with mFAS KS-AT yields. 


\section{Eidesstattliche Erklärung}

Ich erkläre hiermit, dass ich mich bisher keiner Doktorprüfung im mathematisch-naturwissenschaftlichen Bereich unterzogen habe. Des Weiteren erkläre ich, dass ich die vorgelegte Dissertation "Modulating Synthetic Pathways in Megasynthases" selbstständig angefertigt und mich keiner anderen Hilfsmittel als der in ihr angegebenen bedient habe, insbesondere, dass alle Entlehnungen aus anderen Schriften mit Angabe der betreffenden Schrift gekennzeichnet sind.

Ich versichere, die Grundsätze der guten wissenschaftlichen Praxis beachtet und nicht die Hilfe einer kommerziellen Promotionsvermittlung in Anspruch genommen zu haben.

Frankfurt am Main, den 
